University of Louisville

ThinkIR: The University of Louisville's Institutional Repository

Electronic Theses and Dissertations

$5-2020$

\title{
Tobacco product-derived aldehydes: effects on circulating angiogenic cells \& implications for cardiovascular disease.
}

Jordan B. Lynch

University of Louisville

Follow this and additional works at: https://ir.library.louisville.edu/etd

Part of the Cardiovascular Diseases Commons, Environmental Health Commons, and the Toxicology Commons

\section{Recommended Citation}

Lynch, Jordan B., "Tobacco product-derived aldehydes: effects on circulating angiogenic cells \& implications for cardiovascular disease." (2020). Electronic Theses and Dissertations. Paper 3395.

https://doi.org/10.18297/etd/3395

This Doctoral Dissertation is brought to you for free and open access by ThinkIR: The University of Louisville's Institutional Repository. It has been accepted for inclusion in Electronic Theses and Dissertations by an authorized administrator of ThinkIR: The University of Louisville's Institutional Repository. This title appears here courtesy of the author, who has retained all other copyrights. For more information, please contact thinkir@louisville.edu. 
TOBACCO PRODUCT-DERIVED ALDEHYDES: EFFECTS ON CIRCULATING

ANGIOGENIC CELLS \& IMPLICATIONS FOR CARDIOVASCULAR DISEASE

By

Jordan B. Lynch

B.S., McKendree University, 2011

M.S., Southern Illinois University Edwardsville, 2013

M.S., University of Louisville, 2016

A Dissertation

Submitted to the Faculty of the

School of Medicine of the University of Louisville

in Partial Fulfillment of the Requirements

for the Degree of

Doctor of Philosophy

in Pharmacology and Toxicology

Department of Pharmacology and Toxicology

University of Louisville

Louisville, KY

May 2020 

TOBACCO PRODUCT-DERIVED ALDEHYDES: EFFECTS ON CIRCULATING ANGIOGENIC CELLS \& IMPLICATIONS FOR CARDIOVASCULAR DISEASE

By

Jordan B. Lynch

B.S., McKendree University, 2011

M.S., Southern Illinois University Edwardsville, 2013

M.S., University of Louisville, 2016

Dissertation Approved on

April 17th, 2020

by the Following Dissertation Committee:

Daniel J. Conklin, Ph.D.

Aruni Bhatnagar, Ph.D., F.A.H.A.

Gary W. Hoyle, Ph.D.

Rachel Keith, Ph.D.

John P. Wise, Sr., Ph.D. 


\section{DEDICATION}

This dissertation is dedicated to Shelby and Lorene Lashley, whose love and support helped make all my higher schooling possible. 


\section{ACKNOWLEDGEMENTS}

I would like to thank my mentor, Daniel J. Conklin, Ph.D., for all his advice and direction during my time in his lab. I would also like to thank my committee members for their insight and assistance. Additional thanks to Dr. Andrew DeFilippis, Karen Beatty, and Jessica Nystoriak for their advice and assistance in the Louisville Healthy Heart study;

Gregg Shirk, Whitney Theis, and Lexi Hand for assistance with the murine exposures; and Dan Riggs for assistance on statistics. Thanks also to the National Institutes of Health for my funding (ES019217, GM127607, HL122676, HL149351, U54HL120163, T32ES011564).

Many thanks also to my fellow students for their assistance in the lab, but more importantly, for their friendship and advice during my time here. In particular, I would like to thank Dr. Stacey Konkle, Andre Richardson, and Dr. Lexiao Jin for their encouragement and for commiserating with me over the struggles of graduate school.

I would be remiss if I did not thank my parents, Kevin and Lisa Finch. I would never have made it this far without their unfailing support throughout my entire education and especially through these last few years as I worked towards my doctorate. Their examples of dedication and perseverance proved to me that I could succeed, and the fact that they rarely complained about having to hear stories about urine collections proved that they're just the best. And finally, I'd like to thank my husband, Blake, whom I met as a result of said urine collections and who has always believed I could do anything I wanted. It's thanks to him that I've had the courage and optimism to pursue my dreams. 


\section{ABSTRACT \\ TOBACCO PRODUCT-DERIVED ALDEHYDES: EFFECTS ON CIRCULATING ANGIOGENIC CELLS \& IMPLICATIONS FOR CARDIOVASCULAR DISEASE}

Jordan B. Lynch

April 17, 2020

Cardiovascular disease (CVD) is the leading cause of death worldwide. Tobacco smoke is the most significant modifiable risk factor in CVD development and contains numerous toxic compounds, including aldehydes, which have been linked to CVD. Formaldehyde, acetaldehyde, acrolein, and crotonaldehyde have been identified as significant contributors to cigarette-induced health effects, but the mechanism of these effects is not well understood. These aldehydes are also found in aerosols produced by ecigarettes (e-cigs). The goal of this dissertation was to explore the systemic, hematological, and endothelium-related effects of exposure to tobacco products and constituent aldehydes and to identify potential mechanisms of injury. In the Louisville Healthy Heart Study, hematological measures and circulating angiogenic cells (CACs) were assessed against tobacco product use categories. In murine studies, male and female C57BL/6J, transient receptor potential ankyrin 1 (TRPA1)-null, and/or extracellular superoxide dismutasetransgenic (ecSOD-Tg) mice were exposed to mainstream cigarette smoke (MCS), e-cig aerosol, humectants, or constituent aldehydes for varying durations and concentrations. Hematological measures, plasma biomarkers, and levels of CACs were assessed. Chapter III of this dissertation describes findings from the Louisville Healthy Heart Study. E-cig 
or dual users showed significantly increased levels of leukocytes and of CAC-3, an early endothelial progenitor cell population, compared with never smokers. Chapter IV describes systemic and vascular changes induced by exposure to MCS, e-cig aerosol, or humectants, finding significant decreases in leukocytes after all exposures and decreases in CACs in female mice exposed to JUUL Mango aerosol. Chapter V describes effects of exposure to formaldehyde and acetaldehyde, finding that formaldehyde induces significant hematological and vascular changes. Chapter VI describes effects of exposure to acrolein and crotonaldehyde, presenting novel evidence implicating chronic crotonaldehyde exposure as a risk factor for CVD. Finally, chapter VII explores the roles of ecSOD and TRPA1 in tobacco product-related CVD, demonstrating that the effects of unsaturated aldehydes are mediated by TRPA1. This dissertation presents novel data investigating associations between CACs and e-cigs in a human cohort, examining the role of tobacco products and individual constituent aldehydes in the development of CVD, and demonstrating the role of TRPA1 in the CVD effects related to tobacco products. 


\section{TABLE OF CONTENTS}

\begin{tabular}{|c|c|}
\hline & PAGE \\
\hline DEDICATION. & ........iii \\
\hline ACKNOWLEDGEMENTS .. & $\ldots$ iv \\
\hline 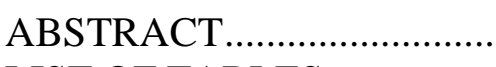 & $\ldots . \mathrm{V}$ \\
\hline LIST OF TABLES ..... & ..xii \\
\hline LIST OF FIGURES . & xvi \\
\hline
\end{tabular}

\section{CHAPTER}

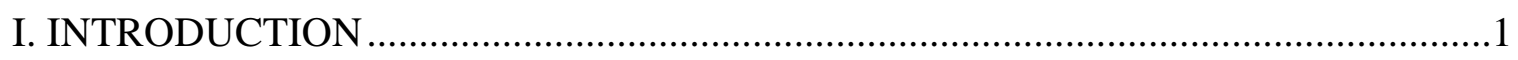

A. Background and Rationale ........................................................................... 1

1. Cardiovascular Disease and Air Pollution.......................................................

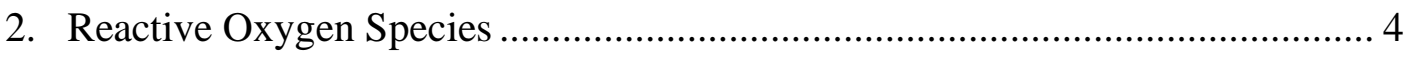

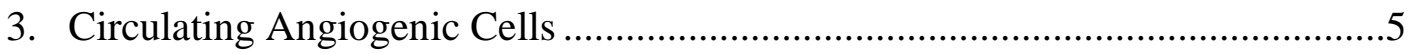

4. Tobacco Smoke and Cardiovascular Disease ...................................................6

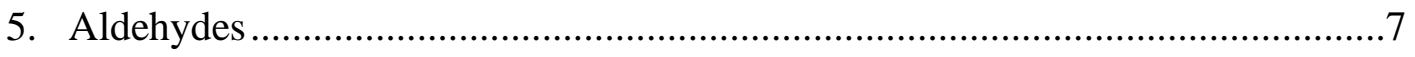

a. Formaldehyde and Acetaldehyde ................................................8

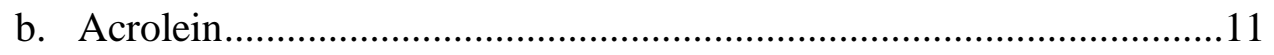

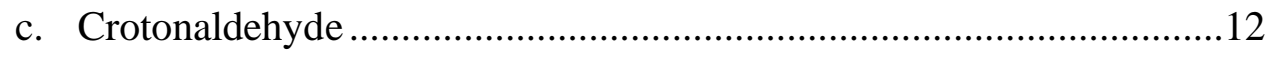

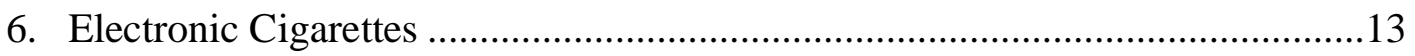

7. Transient Receptor Potential Ankyrin 1 Channel...........................................19

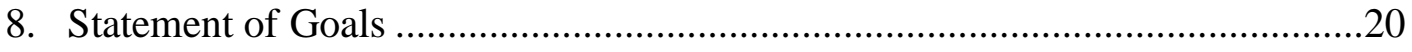

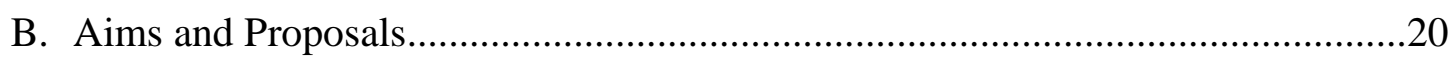


1. Measure Hematological and Endothelium-Related Changes in Humans in Response to Tobacco-Product Related Pollutant Exposures

2. Measure Systemic, Hematological, and Endothelium-Related Changes in Mice in Response to Tobacco Products and Constituent Aldehydes .21

3. Test the Roles of ecSOD and TRPA1 in the Development of Hematological, Systemic, and Endothelium-Related Changes Contributing to CVD

C. Overall Aims of this Dissertation 22

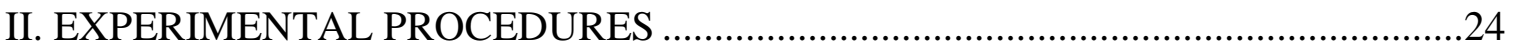

A. Human Studies

1. Study Population .24

2. Eligibility .24

3. Case Definition .25

4. Recruitment .25

5. Questionnaire .26

6. Biological Sample Collection and Processing .26

7. Complete Blood Cell Count (CBC) .27

8. Circulating Angiogenic Cell (CAC) Quantification .27

9. Platelet Mononuclear Cell Aggregate (PMA) Quantification .29

10. Urine Metabolite Quantification .29

a. Creatinine. .29

b. Volatile Organic Compounds (VOCs) and Tobacco Alkaloids .30

11. Statistical Analyses . .30 
B. Murine Studies

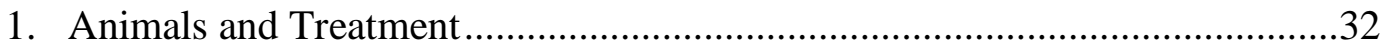

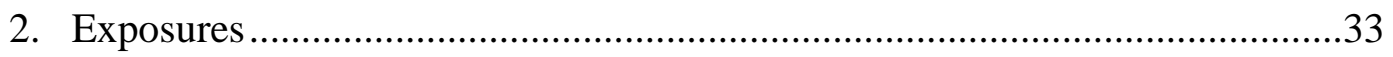

a. Mainstream Cigarette Smoke (MCS)....................................................33

b. Electronic Cigarettes (E-cigs) ………………………………….........3

c. Propylene Glycol and Vegetable Glycerin (PG:VG)............................34

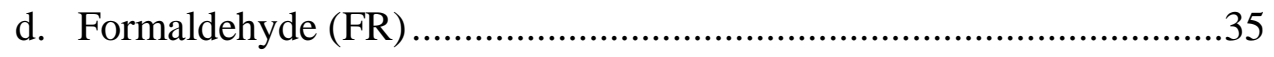

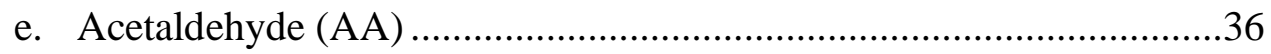

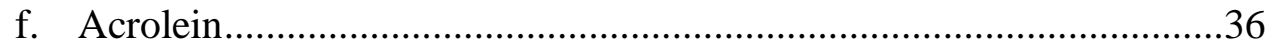

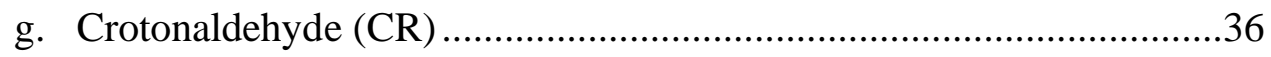

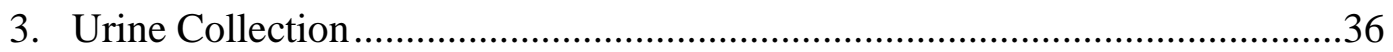

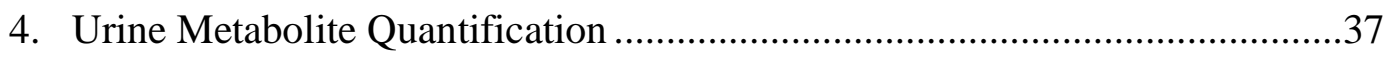

a. Formate and Acetate ….......................................................................

b. Volatile Organic Compounds (VOCs) and Tobacco Alkaloids.............37

5. Tissue Collection and Storage …………………….......................................

6. Complete Blood Cell Count (CBC) ……………...........................................39

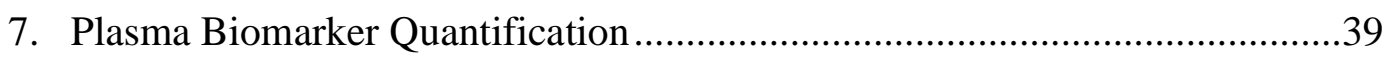

8. Circulating Angiogenic Cell (CAC) Quantification ...........................................39

9. Leukocyte Subpopulation Quantification ……………....................................40

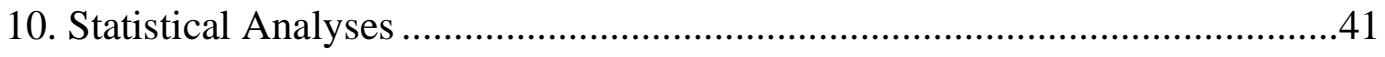

\section{ASSOCIATIONS BETWEEN CIRCULATING ANGIOGENIC CELLS AND USE} OF TRADITIONAL AND EMERGING TOBACCO PRODUCTS............................42

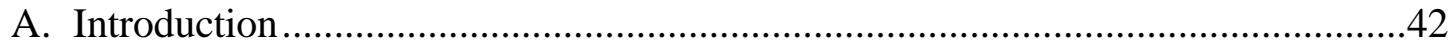




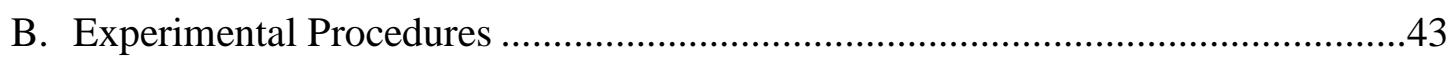

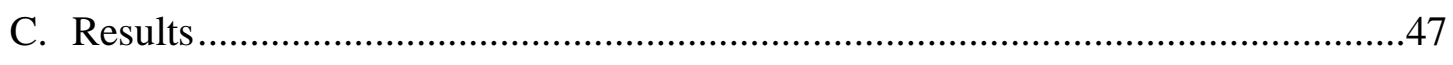

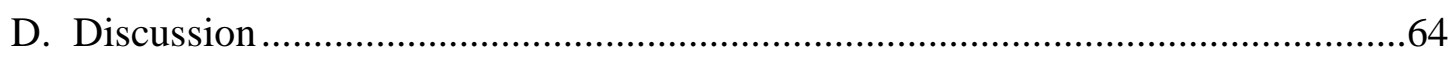

IV. SYSTEMIC, HEMATOLOGICAL, AND ENDOTHELIUM-RELATED EFFECTS OF MAINSTREAM CIGARETTE SMOKE, E-CIGARETTE AEROSOL, AND PROPYLENE GLYCOL:VEGETABLE GLYCERIN IN MICE .................................74

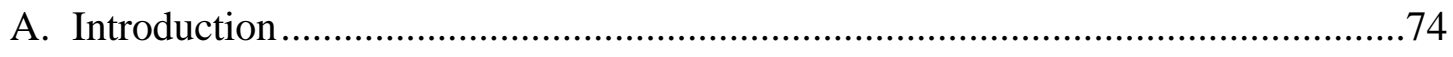

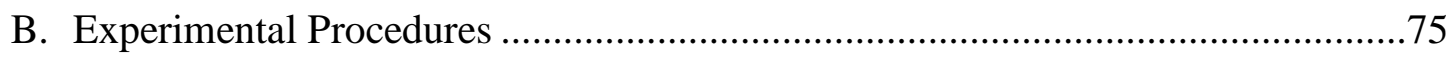

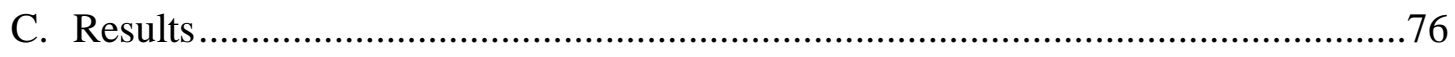

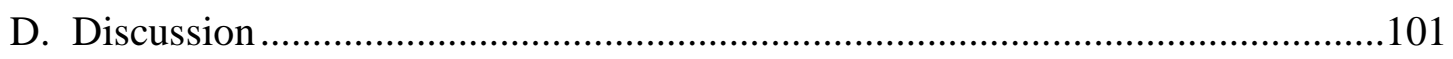

V. SYSTEMIC, HEMATOLOGICAL, AND ENDOTHELIUM-RELATED EFFECTS OF FORMALDEHYDE AND ACETALDEHYDE IN MICE ..................................109

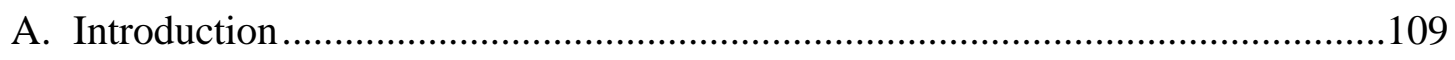

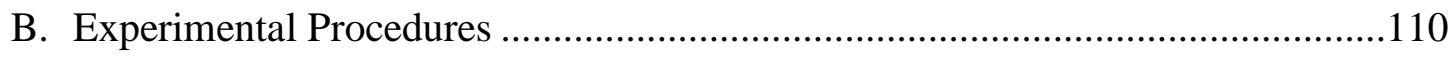

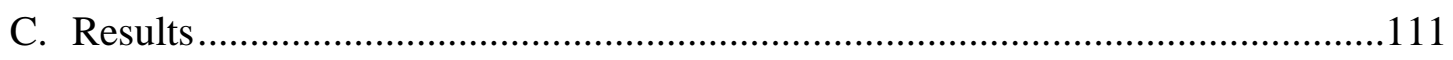

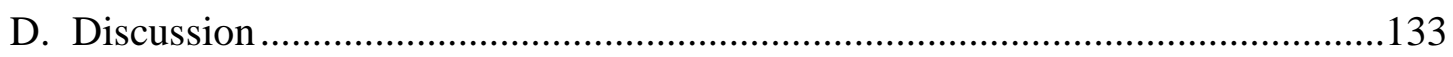

VI. SYSTEMIC, HEMATOLOGICAL, AND ENDOTHELIUM-RELATED EFFECTS OF ACROLEIN AND CROTONALDEHYDE IN MICE .........................................143

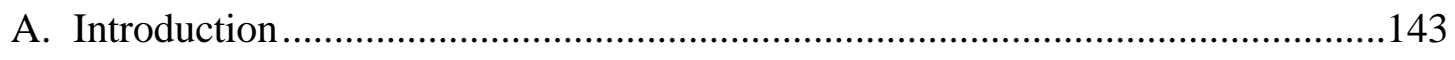

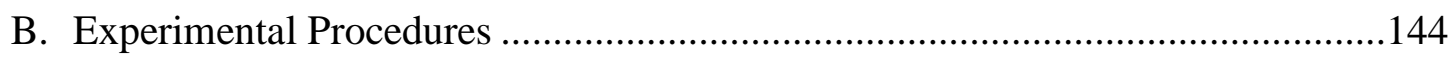

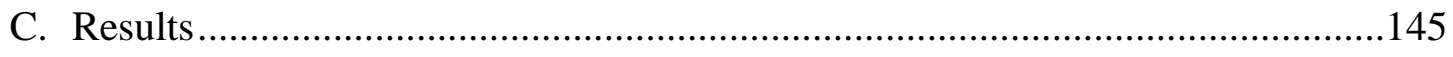

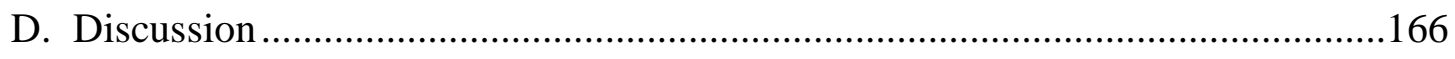




\section{ROLES OF EXTRACELLULAR SUPEROXIDE DISMUTASE AND}

TRANSIENT RECEPTOR POTENTIAL ANKYRIN 1 IN THE

\section{CARDIOVASCULAR EFFECTS OF TOBACCO PRODUCTS AND} CONSTITUENT ALDEHYDES

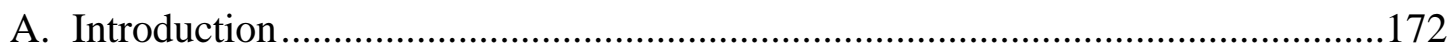

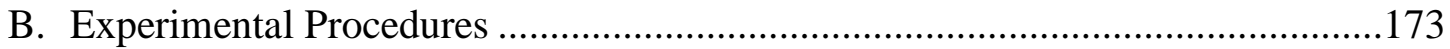

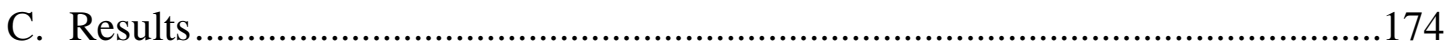

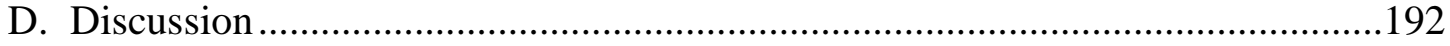

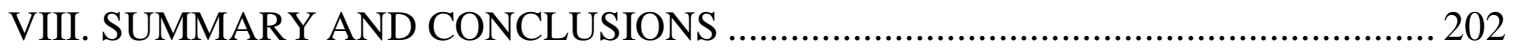

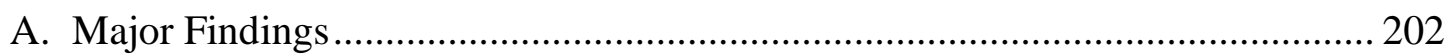

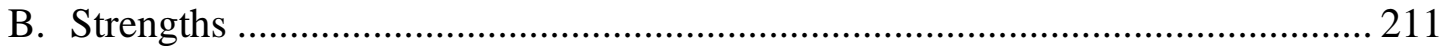

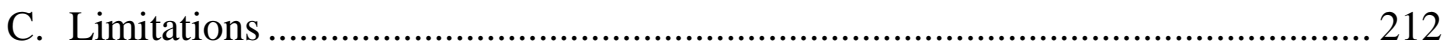

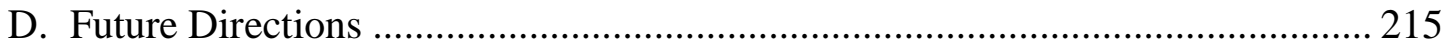

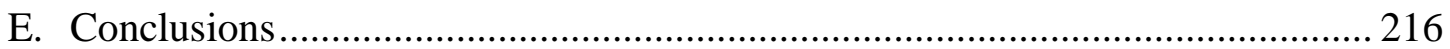

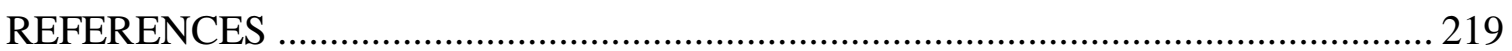

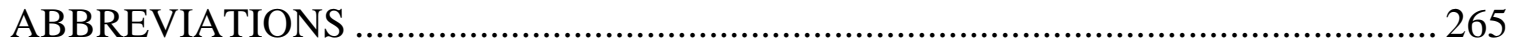

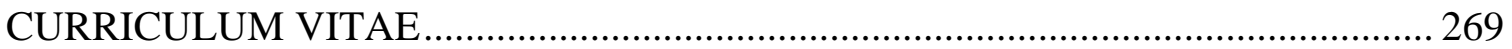




\section{LIST OF TABLES}

TABLE

PAGE

Table 3.1. Antigenic identity of circulating angiogenic cells (CACs) .46

Table 3.2. Demographic characteristics by tobacco product use categories .48

Table 3.3. Medical history and medication use by tobacco product use categories .51

Table 3.4. Hematological measures by tobacco product use categories.

Table 3.5. Mean urinary volatile organic compound (VOC) metabolite levels by tobacco product use categories (ng/mg)

Table 3.6. Percent chance in levels of circulating angiogenic cells (CACs) comparing tobacco product use categories with never smokers in the fully adjusted model

Table 3.7. Percent change in levels of circulating angiogenic cells (CACs) comparing tobacco product use categories with never smokers in the secondary adjusted model .....61 Table 4.1. Systemic parameters of male C57BL/6 mice acutely exposed to either air or mainstream cigarette smoke (MCS; 50\% of smoke of $12 \mathrm{KY}$ Reference 3R4F cigarettes)

Table 4.2. Hematological measures of male C57BL/6 mice acutely exposed to either air or mainstream cigarette smoke (MCS; $50 \%$ of smoke of $12 \mathrm{KY}$ Reference 3R4F

cigarettes)

Table 4.3. Systemic parameters of female C57BL/6 mice acutely exposed to either air or electronic cigarette aerosol (e-cig; JUUL Mango) .88 
Table 4.4. Hematological measures of female C57BL/6 mice acutely exposed to either air or electronic cigarette aerosol (e-cig; JUUL Mango)

Table 4.5. Systemic parameters of male C57BL/6 mice acutely exposed to either air or

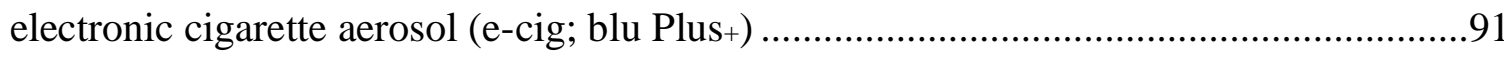

Table 4.6. Hematological measures of male C57BL/6 mice acutely exposed to either air

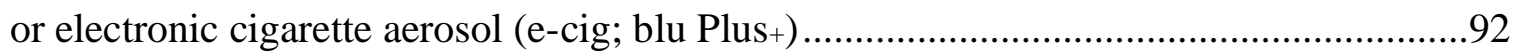

Table 4.7. Systemic parameters of female C57BL/6 mice acutely exposed to either air or propylene glycol:vegetable glycerin (PG:VG; 30\%:70\%) .98

Table 4.8. Hematological measures of female C57BL/6 mice acutely exposed to either air or propylene glycol:vegetable glycerin (PG:VG; 30\%:70\%)

Table 5.1. Systemic parameters of male C57BL/6 mice acutely exposed to either air or formaldehyde (FR; 1, 2, or $5 \mathrm{ppm})$

Table 5.2. Hematological measures of male C57BL/6 mice acutely exposed to either air or formaldehyde (FR; 1, 2, or $5 \mathrm{ppm})$ 116 Table 5.3. Systemic parameters of female C57BL/6 mice acutely exposed to either air or formaldehyde (FR; 2 or 5 ppm) .121

Table 5.4. Hematological measures of female C57BL/6 mice acutely exposed to either air or formaldehyde (FR; 2 or 5 ppm)

Table 5.5. Systemic parameters of male C57BL/6 mice acutely exposed to either air or acetaldehyde (AA; 5 ppm) 130 Table 5.6. Hematological measures of male C57BL/6 mice acutely exposed to either air or acetaldehyde (AA; 5 ppm). 131 
Table 5.7. Systemic changes in female C57BL/6 mice acutely exposed to either air or acetaldehyde (AA; 5 ppm)

Table 6.1. Systemic parameters of male C57BL/6 mice acutely exposed to either air or acrolein $(1 \mathrm{ppm})$

Table 6.2. Hematological measurements of male C57BL/6 mice acutely exposed to either air or acrolein $(1 \mathrm{ppm})$ 150

Table 6.3. Systemic parameters in male C57BL/6J mice chronically exposed to either air or crotonaldehyde (CR; $1 \mathrm{ppm})$

Table 6.4. Hematological measures of male C57BL/6J mice chronically exposed to either air or crotonaldehyde (CR; $1 \mathrm{ppm})$

Table 6.5. Systemic measures in male C57BL/6J mice acutely exposed to either air or crotonaldehyde (CR; 1 or $3 \mathrm{ppm})$

Table 6.6. Plasma biomarkers in male C57BL/6J mice acutely exposed to either air or crotonaldehyde (CR; 1 or $3 \mathrm{ppm})$

Table 6.7. Systemic measures in female C57BL/6J mice acutely exposed to either air or crotonaldehyde (CR; $1 \mathrm{ppm})$.

Table 7.1. Systemic parameters of male TRPA1-null mice acutely exposed to either air or mainstream cigarette smoke (MCS; $50 \%$ of smoke of $12 \mathrm{KY}$ Reference 3R4F cigarettes)

Table 7.2. Hematological measures of male TRPA1-null mice acutely exposed to either air or mainstream cigarette smoke (MCS; $50 \%$ of smoke of $12 \mathrm{KY}$ Reference 3R4F cigarettes) 
Table 7.3. Systemic measures in male TRPA1-null mice acutely exposed to either air or crotonaldehyde (CR; $1 \mathrm{ppm})$.......

Table 7.4. Plasma biomarkers in male TRPA1-null mice acutely exposed to either air or crotonaldehyde (CR; $1 \mathrm{ppm})$. .181

Table 7.5. Systemic changes in male WT or ecSOD-Tg mice acutely exposed to either air or mainstream cigarette smoke (MCS; 50\% of smoke of 6 KY Reference 3R4F cigarettes)

Table 7.6. Systemic changes in male WT or ecSOD-Tg mice acutely exposed to either air or mainstream cigarette smoke (MCS; 50\% of smoke of $12 \mathrm{KY}$ Reference 3R4F cigarettes)

Table 7.7 Plasma biomarkers in male WT or ecSOD-Tg mice acutely exposed to either air or mainstream cigarette smoke (MCS; $50 \%$ of smoke of $6 \mathrm{KY}$ Reference 3R4F cigarettes)

Table 7.8. Plasma biomarkers in male WT or ecSOD-Tg mice acutely exposed to either air or mainstream cigarette smoke (MCS; 50\% of smoke of $12 \mathrm{KY}$ Reference 3R4F cigarettes) .194 


\section{LIST OF FIGURES}

FIGURE

PAGE

Figure 3.1. Flow cytometry gating scheme for circulating angiogenic cell (CAC)

populations

Figure 3.2. Relative feature importance of demographic variables on levels of circulating angiogenic cells (CACs)

Figure 3.3. Interrelationships between circulating angiogenic cells (CACs) associated with e-cig or dual use compared with never smokers

Figure 4.1. Urinary metabolites after acute mainstream cigarette smoke (MCS) exposure

Figure 4.2. Effects of acute mainstream cigarette smoke (MCS) exposure on circulating stem cells in male mice. .82

Figure 4.3. Urinary metabolites after acute electronic cigarette aerosol (e-cig; JUUL VA

Tobacco) exposure .84

Figure 4.4. Urinary metabolites after acute electronic cigarette aerosol (e-cig; JUUL

Mango) exposure

Figure 4.5. Urinary metabolites after acute electronic cigarette aerosol (e-cig; JUUL

Menthol) exposure

Figure 4.6. Effects of acute electronic cigarette (e-cig; JUUL Mango) aerosol exposure

on circulating stem cells in female mice. 
Figure 4.7. Effects of acute electronic cigarette (e-cig; blu Plus+) aerosol exposure on circulating stem cells in male mice

Figure 4.8. Urinary metabolites after acute propylene glycol:vegetable glycerin (PG:VG; $30 \%: 70 \%$ ) exposure .95

Figure 4.9. Urinary metabolites after acute propylene glycol:vegetable glycerin (PG:VG; 50\%:50\%) exposure .96

Figure 4.10. Effects of acute propylene glycol:vegetable glycerin (PG:VG; 30\%:70\%) exposure on circulating stem cells in female mice 100

Figure 5.1. Urinary formate levels in formaldehyde- or tobacco product-exposed male mice

Figure 5.2. Effects of two-week formaldehyde (FR; 5 ppm) exposure on circulating stem cells in male mice 118

Figure 5.3. Effects of acute formaldehyde (FR; $5 \mathrm{ppm})$ exposure on circulating stem cells in male mice

Figure 5.4. Effects of acute formaldehyde (FR; $2 \mathrm{ppm})$ exposure on circulating stem cells in male mice 124 Figure 5.5. Effects of acute formaldehyde (FR; $2 \mathrm{ppm})$ exposure on circulating stem cells in female mice 125 Figure 5.6. Effects of acute formaldehyde (FR; $5 \mathrm{ppm}$ ) exposure on circulating stem cells in female mice 126 Figure 5.7. Urinary acetate levels in acetaldehyde- or tobacco product-exposed male mice .128 
Figure 5.8. Effects of two-week acetaldehyde (AA; 5 ppm) exposure on circulating stem cells in male mice

Figure 5.9. Effects of acute acetaldehyde (AA; $5 \mathrm{ppm})$ exposure on circulating stem cells in male mice.

Figure 5.10. Effects of acute acetaldehyde (AA; $5 \mathrm{ppm})$ exposure on circulating stem cells in female mice 136

Figure 6.1. Urinary 3HPMA levels in acrolein- or tobacco product-exposed male mice

Figure 6.2. Effects of acute acrolein $(1 \mathrm{ppm})$ exposure on circulating stem cells in male

mice .151

Figure 6.3. Urinary HPMMA levels in crotonaldehyde- or tobacco product-exposed male mice

Figure 6.4. Effects of chronic crotonaldehyde (CR; $1 \mathrm{ppm})$ exposure on circulating stem cells in male mice 158

Figure 6.5. Effects of acute crotonaldehyde (CR; $1 \mathrm{ppm})$ exposure on circulating stem cells in male mice .164

Figure 6.6. Effects of acute crotonaldehyde (CR; 3 ppm) exposure on circulating stem cells in male mice 165

Figure 6.7. Effects of acute crotonaldehyde (CR; $1 \mathrm{ppm})$ exposure on circulating stem cells in female mice 167 Figure 7.1. Effects of acute crotonaldehyde (CR; $1 \mathrm{ppm})$ exposure on circulating stem cells in male TRPA1-null mice 183 
Figure 7.2. Urinary 3HPMA \& HPMMA levels after acute mainstream cigarette smoke

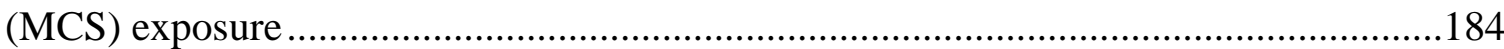

Figure 7.3. Total urinary nicotine metabolite levels after acute mainstream cigarette

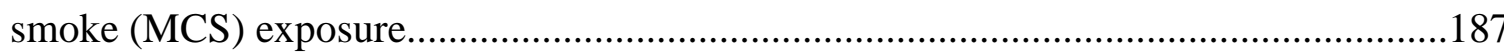

Figure 7.4. Effects of acute mainstream cigarette smoke (MCS; 6 or 12 cigarettes) exposure on circulating stem cells in male WT and ecSOD-Tg mice ...........................195 


\section{CHAPTER I}

\section{INTRODUCTION}

\section{A. Background and Rational}

\section{Cardiovascular Disease and Air Pollution}

Cardiovascular disease (CVD) is the leading cause of death both nationally (1) and globally (2). In 2016, the World Health Organization reported that approximately $27 \%$ of the 56.9 million deaths worldwide were due to ischemic heart disease and stroke; these diseases have been the leading causes of death globally for the last 20 years (2). In the United States, the American Heart Association reports that approximately 1 of every 3 deaths is due to CVD, with about 2,300 daily deaths related to some form of CVD (1). On a local level, between 2014-2016, Kentucky had a CVD mortality rate of 258.5 per 100,000 people, exceeding the national CVD mortality rate of 220.7 per 100,000 people by approximately $17 \%$ and ranking the state as the fifth highest in the nation for CVD mortality (1). The American Heart Association projects that CVD prevalence will continue to increase, with approximately $45.1 \%$ of the United States population expected to have some form of CVD by 2035 (1). In terms of cost, the average annual direct and indirect cost of CVD-related care in the United States in 2014 to 2015 was $\$ 351,200,000,000$, an amount which accounted for $14 \%$ of the total health expenditures for the county in that 
year (1). As CVD prevalence increases, the cost of CVD-related health care is also expected to increase, with estimates of total direct CVD-related costs of approximately $\$ 749,000,000,000$ per year by 2035 (1).

Although there are many risk factors for the development of CVD (3), a better understanding of the relationship between environmental factors and health outcomes has led to the hypothesis that environmental exposures to air pollutants contribute to CVD development. Over the past 30 years, a number of clinical and epidemiological studies have established the damaging effects of air pollution on human health. The majority of this damage is found in the pulmonary and cardiovascular systems, leading to an increased risk of heart disease or stroke (4-13). Many of these health effects are linked to different forms of air pollution, including ambient air pollution and diesel and gasoline engine exhausts. Ambient air pollution is composed of a gaseous suspension of aerosolized particles, known as particulate matter (PM), that typically have an aerodynamic diameter $<10 \mu \mathrm{m}(14,15)$; this range is classified into specific particle sizes: ultrafine $(<0.1 \mu \mathrm{m})$, fine $\left(\mathrm{PM}_{2.5}, 0.1-2.5 \mu \mathrm{m}\right)$, and coarse (PM10, >2.5-10 $\left.\mu \mathrm{m}\right)$. General exposure to ambient air pollution has been shown to be a significant trigger for heart attacks (4), and there is strong evidence linking increased levels of PM2.5 with increased cardiovascular effects (15-17). A $10 \mu \mathrm{g} / \mathrm{m}_{3}$ increase in $\mathrm{PM}_{2.5}$ levels is associated with an increased risk of heart failure hospitalization (18) and with long-term all-cause mortality (5-9).

Additional studies of specific types of air pollutants further strengthen the link between exposure and cardiovascular events. PM2.5 exposure significantly increases left ventricular mass $(10)$ and systolic $(11,19,20)$ and mean ambulatory arterial (21) blood pressures. Changes in PM2.5 are associated with the onset of heart attacks (22) and 
increased intima media thickness progression $(12,13)$, with accelerated carotid intima media thickness (CIMT) progression of up to $5.5 \mu \mathrm{m}$ (per $10 \mu \mathrm{g} / \mathrm{m}_{3}$ ) per year (23). Similarly, exposure to diesel exhaust and traffic-related pollution increases arterial stiffness (24) and systolic blood pressure (25) and is associated with both increased mortality hazard ratio and increased risk of dying after hospital discharge (26). Collectively, these pathogenic changes (e.g., increased CIMT, increased blood pressure) suggest that an underlying vascular alteration or dysfunction is responsible, and thus, there is a need to explore mechanisms consistent with these vascular manifestations.

Brook et al. (16) propose three likely mechanisms by which air pollutants may contribute to the development of CVD: 1) an imbalance in the autonomic nervous system; 2) translocation of particles, specifically ultrafine particulate matter, into the circulation; and, 3) the development of systemic oxidative stress and inflammation within the vasculature. These hypothetical pathways are generally accepted $(17,21,24,27-32)$. A primary outcome of each of these mechanisms is the initiation of endothelium dysfunction, which appears central (perhaps as a result of or in combination with oxidative stress and inflammation) to further development of CVD. Evidence suggests a strong association between endothelium dysfunction and cardiovascular events, leading to high morbidity and mortality from CVD (33) as well as other disease states (34-39). Nonetheless, more research is required in order to ultimately improve pollutant-related health outcomes. Based on the health and financial burden of CVD, it is necessary from medical, legislative, and economic standpoints to continue working towards decreased air pollutant exposures in an effort to collectively reduce morbidity and mortality and to reveal the biological mechanism(s) by which air pollutants cause and/or exacerbate CVD. 


\section{Reactive Oxygen Species}

Oxidative stress as proposed by Brook et al. (16) is the result of excessive production of reactive oxygen species (ROS) (40-42). ROS are oxygen-containing, highly reactive molecules, many of which are endogenously produced by normal cellular metabolism $(42,43)$. At normal levels, ROS have been shown to have a number of beneficial aspects, serving as signaling molecules $(43,44)$ and playing roles in gene expression, cell growth, and apoptosis $(42,43)$. However, stressors such as pollutants or toxicants can lead to increased production of $\operatorname{ROS}(32,45,46)$, overcoming the body's ability to clear these oxidizing compounds and leading to the development of oxidative stress $(40-42,44,47)$. Increased ROS and the resulting oxidative stress have been linked to a number of negative health outcomes $(42,47,48)$, including CVD $(42,48-52)$.

Increased ROS and oxidative stress have been linked to downstream health outcomes via changes in the production and bioavailability of nitric oxide (NO), a potent vasodilator $(40-42,49)$. Excess levels of the anion superoxide in particular have been directly implicated in disease development via the development of endothelium dysfunction (40-42). These excess levels can occur when superoxide dismutase (SOD), the antioxidant enzyme that catalyzes the dismutation of superoxide, is inactivated or mutated (53). The free superoxide molecule can then bind with NO to form peroxynitrite, a powerful oxidant that can subsequently cause dysfunction of endothelial nitric oxide synthase (eNOS), one of the enzymes responsible for synthesizing $\mathrm{NO}(40,41,49,50)$. By disrupting eNOS function, NO levels are further decreased, leading to an imbalance in the vasoactive factors in the vasculature and resulting in endothelium dysfunction $(40,41)$. This dysfunction has been noted as one of the earliest vascular changes in a number of 
disease states, including atherosclerosis (54-56) and both systemic (57) and pulmonary (58) hypertension. Although a number of therapeutic strategies specifically targeting the production of ROS and the development of endothelium dysfunction have been developed for the treatment of CVD (59-61), the relationship between these endothelial and vascular changes and downstream disease development is complex, and more research is needed to fully understand these connections.

\section{Circulating Angiogenic Cells}

Once endothelium dysfunction develops, repair is at least partially mediated by circulating angiogenic cells (CACs) $(62,63)$. CACs are sensitive indicators of both vascular health and pollution exposure, and they play an important role in proper endothelial function and overall endothelial health (63-66). These cells are recruited from the bone marrow to sites of endothelial damage where they contribute to repair of the vasculature $(62,67-69)$. Vasa et al. (70) found that the number of atherosclerotic risk factors, such as hypertension, diabetes, and hypercholesterolemia, was inversely correlated with CAC levels and CAC function. Similarly, Hill et al. (66) demonstrated a strong correlation between an individual's Framingham Risk Score, the 10-year risk score for nonfatal heart attack or sudden cardiac death, and their CAC levels, with higher risk scores associated with diminished CAC levels. Furthermore, the group showed a significant relationship between endothelial function as measured by flow-mediated brachial reactivity and CACs (66).

Based on these results, Hill et al. concluded that CACs were a better predictor of vascular reactivity than the presence or absence of conventional risk factors and that CACs may serve as a surrogate biologic marker for vascular function and cumulative 
cardiovascular risk (66). This concept has been evaluated in a number of studies examining the relationship between CVD and air pollution. Consistent with Hill's theory, these studies show that exposure to air pollution causes a decrease in CAC levels that correlates with an increased CVD risk (67, 71-74). Therefore, it is imperative for any research concerning CVD and air pollution to assess CAC levels and function in relation to endothelium dysfunction and as an indicator of vascular homeostasis.

\section{Tobacco Smoke and Cardiovascular Disease}

It is also important to consider personal sources of pollutant exposures when conducting CVD research. While some level of exposure to ambient air pollution is inevitable (4), exposure to indoor air pollutants can be controlled to a degree; one indoor air pollutant that is of particular health concern is tobacco smoke. Tobacco smoke is the most significant modifiable risk factor in the development of CVD (1, 14, 75-77). Cigarettes contains numerous toxic compounds, many of which could contribute to disease development (30, 78-82). The Centers for Disease Control and Prevention (CDC) reports that although cigarette use has been declining for a number of years, reaching an all-time low of $13.7 \%$ in 2018 (83), tobacco use still remains the leading cause of preventable death both globally and nationally, with overall mortality for American smokers three times that of their fellow non-smokers (1). Furthermore, secondhand smoke (SHS) has also been shown to cause a large number of negative health effects. The CDC reports that since 1964, approximately two and a half million nonsmokers have died from health problems caused by exposure to SHS (84). The Surgeon General's report estimates more than 33,000 nonsmokers die every year in the United States from coronary heart disease caused by exposure to SHS (80). It has also been shown that SHS exposure can cause heart attacks 
and strokes in nonsmokers $(80,84)$, and a variety of health consequences in both children and adults have been linked to SHS exposure as well, including cardiovascular, respiratory, and reproductive effects $(80,84)$. Much as with ambient air pollutant exposure, the development of oxidative stress and endothelium dysfunction likely contributes to this disease development, as both mainstream cigarette smoke (MCS) $(85,86)$ and SHS (87, 88) exposures have been linked to increased production of ROS.

\section{Aldehydes}

Aldehydes, one class of compounds known as volatile organic compounds (VOCs), are compounds with a terminal carbonyl group (-CHO), and they can be classified as saturated, unsaturated, or aromatic. Unsaturated aldehydes contain one or more carboncarbon double bond, which increase their reactivity compared with saturated aldehydes (89). These compounds are generally ubiquitous in the natural environment, and many are found in foods and drinks, including meats, cheeses, fried foods, bread, and beer and other alcohols (90). However, the majority of environmental aldehydes are generated from anthropogenic sources, including combustion sources (e.g., diesel and gasoline exhaust, industrial emissions, MCS, and SHS) and also various consumer products and building materials that leach aldehydes (90). Additionally, as they have been linked to a number of negative health effects (91-94), aldehydes from both ambient and personal exposure

sources (e.g., MCS, SHS, occupational exposures, personal hobby exposures) present potential health risks. Because of their pervasiveness and their documented toxicity, four specific aldehydes have been identified as significant contributors to disease risk and development: formaldehyde, acetaldehyde, acrolein, and crotonaldehyde. 


\section{a. Formaldehyde and Acetaldehyde}

The cigarette- and pollutant-related development of CVD may in part be attributable to saturated aldehydes such as formaldehyde (FR) and acetaldehyde (AA). Both of these aldehydes have been officially established by the United States Food and Drug Administration as harmful constituents in tobacco products and tobacco smoke, with FR listed as a respiratory toxicant and a carcinogen and AA listed as a carcinogen, a respiratory toxicant, and an addictive component $(82,93,95)$. Furthermore, the Institute of Medicine ranked AA and FR as two of the most significant toxins in tobacco smoke in relation to CVD (96). There is some level of natural exposure to both of these products, both exogenously and endogenously. FR, the smallest aldehyde, is naturally produced in the cells of humans and other mammals by amino acids and via the demethylation of various endogenous metabolites $(93,97,98)$. It is swiftly degraded via FR dehydrogenase and S-formylglutathione hydrolase to form formic acid $(93,97,99)$ and does not therefore accumulate in the blood, with a half-life of approximately 1.5 minutes $(99,100)$, contributing to a basal concentration of $2-3 \mathrm{mg} / \mathrm{L}(93,97,99)$. In terms of exogenous sources, FR is released as a combustion product, both of biomass (101) and fuel $(102,103)$. Combustion accounts for the majority of FR released in the environment (102). FR is also used in many consumer products, including carpets, manufactured wood products, paints and varnishes, and preservatives, which may lead to increased exposure (102). However, levels of FR in the air are typically low, $(100,102,104)$, with natural concentrations less than $1 \mu \mathrm{g} / \mathrm{m}_{3}(93,99)$, although some variation is seen between rural, suburban, and urban locations $(93,99,102)$.

AA, the second smallest aldehyde, is pervasive in the ambient environment and is 
widely used in different industries as an intermediate in the production of other chemicals $(105,106)$. It is produced due to the incomplete combustion of wood from both personal and industrial use $(105,106)$, and it is also naturally found in dairy, meats, and plants commonly consumed by humans (105). Alcohol consumption is another common source of exposure $(105,106)$, as AA is formed from the breakdown of ethanol via alcohol dehydrogenases. The concentration in ambient air is typically between 5 and $10 \mu \mathrm{g} / \mathrm{m} 3$ $(92,107)$. Like FR, AA has a short half-life in the blood — again, approximately 90 seconds (108, 109) — and does not typically accumulate in the blood; Lynch et al. report the concentration of AA in the whole blood of fasted subjects to be less than $0.1 \mathrm{mg} / \mathrm{L}$ (110).

Although some exposure to both FR and AA is common for all individuals, those exposed to MCS or SHS are exposed to much higher levels of these compounds, greatly increasing their risk of morbidity and mortality $(92,93,95,99,111-113)$. The World Health Organization reports that, on average, a person is naturally exposed to approximately $1 \mathrm{mg}$ of FR per day; smoking 20 cigarettes per day will increase that exposure, raising levels of inhaled FR by an additional 0.9-2.0 mg/day (99). Direct analysis of MCS has measured levels of FR between 12-29 $\mu \mathrm{g}$ /cigarette (114-117), and additional exposure from SHS can increase FR exposure by $0.2-3.5 \mathrm{mg} /$ day taking into account exposure possible in both the workplace and at home (99).

Typical exposure to AA, although ubiquitous, occurs via food intake, particularly alcohol consumption (105-107). Therefore, any significant level of exposure via inhalation can significantly increase overall exposure, and tobacco smoke is one of the most common modifiable forms of exposure to AA $(107,118)$. Based on the mean ambient concentrations of AA in both indoor and outdoor environments, Bruinen de Bruin et al. 
(107) estimate that an individual is exposed to $0.321 \mathrm{mg}$ of AA a day. The current literature repots a range of AA within cigarettes, but most data suggest between $0.5-3 \mathrm{mg} /$ cigarette $(107,109,114,117,118)$. Based on 2016 data showing that American daily smokers consume an average of 14 cigarettes per day - a level that is down from the estimated 17 cigarettes per day in 2005- this increases the average smoker's exposure to AA by 7-42 mg/day (119). Exposure to AA via SHS may be equivalent or even greater than that from MCS (107, 118), with reported levels ranging from 2-3 mg/cigarette (107).

Some cardiovascular and hematopoietic effects of FR or AA exposure are documented, but there remain questions regarding the mechanisms through which these aldehydes cause cardiovascular effects. Exposure to FR has been shown to cause hematotoxicity, significantly decreasing total leukocyte count $(93,120-122)$ as well as platelets and red blood cell counts $(93,120,122)$. Progenitor cell lines were additionally seen to be decreased with exposure $(120,122)$. FR has also been shown to cause decreases in blood pressure; in rats, inhalation of formalin, a saturated solution of FR in water, produced a significant drop in arterial blood pressure (123), and in vitro studies also note a concentration-dependent relaxation in response to FR (124-127). Hypotension has also been noted in individuals who have attempted suicide via ingestion of FR (128). Furthermore, occupational exposures in industries associated with increased exposure to FR (embalmers, undertakers, perfumery workers) have been associated with increased incidence of heart disease (129-131).

Human studies of the cardiovascular effects of AA typically revolve around aldehyde dehydrogenase (ALDH2), the fast-acting enzyme that metabolizes AA, and specifically the mutation in the enzyme that prevents AA metabolism and allows for 
increased blood levels of AA (132). This mutation is present in 35-45\% of Asians and causes facial flushing and nausea after alcohol consumption, presumably due to high levels of AA in the blood (133). The resulting increase in AA has been linked to several negative cardiovascular outcomes, including increased risk of hypertension $(134,135)$, coronary heart disease $(136,137)$, and heart attack $(136)$, as well as alterations in heart rhythm $(132$, $138)$ and overall cardiac toxicity $(132,135)$. Other studies have observed that prolonged exposure to AA can cause decreases in both leukocytes and red blood cells (139), and the formation of hemoglobin-AA adducts decreases the amount of free hemoglobin available in the blood, which could contribute to anemia (139-142).

\section{b. Acrolein}

Unsaturated aldehydes, aldehydes that contain at least one carbon-carbon double bond, have also been linked to the development of CVD $(71,74,86,143-145)$. Unsaturated aldehydes are more reactive than their saturated counterparts as a result of the polarization of their carbon-carbon double bond by the aldehyde group, which increases the potential for nucleophilic addition (146). One unsaturated aldehyde that has been extensively researched is acrolein. Acrolein is a combustion air pollutant associated with tobacco smoke, vehicular exhaust, open fires, and industrial emissions (94). Although acrolein can be found in the environment, levels are typically low, and the highly reactive nature of the compound gives acrolein a half-life of roughly one day (94). Exposure more commonly occurs via ingestion of foods such as beer, cheese, bread, coffee, and fried or burnt foods, and some level of endogenous production of acrolein occurs via lipid peroxidation (94). Although both ambient and indoor levels of acrolein are typically low, ranging between $<1$

and $12 \mathrm{ppb}$, indoor levels can become significantly increased by tobacco smoke. MCS is 
known to be a significant source of acrolein, with between 18-98 $\mu \mathrm{g} /$ cigarette or 50-70 ppm of acrolein generated per puff in MCS $(90,114,117,147)$; this level is significantly higher than the 4-8 ppm generated by exhaust from gasoline and diesel engines (148).

The Agency for Toxic Substances and Disease Registry classifies acrolein as an eye, nose, skin, and respiratory irritant, and the United States Department of Health and Human Services notes that acrolein may be a possible human carcinogen (94). Acrolein has been shown to also contribute to the development of CVD. Haussmann (145) reports that almost $90 \%$ of the non-cancer health risk associated with smoking, particularly the risk of cardiovascular and pulmonary diseases, is thought to be due to acrolein, and Stabbert et al. (149) have shown that the majority of the cytotoxicity related to the gas vapor phase constituents of MCS is due to acrolein. Furthermore, exposure to acrolein has been linked to increases in CVD risk in both humans and mice $(71,74,150-153)$; inhalation of acrolein even at levels low enough to not induce a respiratory response showed changes in cardiac performance (154). Inhalation of acrolein has also been shown to cause endothelium dysfunction in mice (150) and to decrease levels of CACs in both humans (71) and murine models $(74,151)$, while ingestion of acrolein promotes pro-atherosclerotic changes $(152$, 153).

\section{c. Crotonaldehyde}

Crotonaldehyde (CR) is another unsaturated aldehyde found in high levels in MCS and linked to CVD. CR is found in many foodstuffs, including meat, fruits, vegetables, milk, and bread $(155,156)$, and there is some endogenous production of CR via lipid peroxidation and the metabolism of 1,3-butadiene (157). However, most exposure to CR occurs through anthropogenic sources such as industry emissions, engine exhaust, wood 
smoke, and tobacco smoke (155-157). Because CR is a ubiquitous compound, low levels of exposure via ingestion or inhalation are expected on a day-to-day basis (158), but most exposure occurs via cigarette smoke $(156,159)$. The concentration of CR in MCS is estimated to be between $1-53 \mu \mathrm{g} /$ cigarette, although this level may vary somewhat based on the type of cigarette (160). However, a recent study by Jaccard et al. (117) reported a CR yield of approximately $10 \mu \mathrm{g} /$ cigarette in 3R4F Kentucky reference cigarettes, which are commonly used as standard cigarettes for non-clinical tobacco research (161).

$\mathrm{CR}$, like acrolein, is classified as an eye, nose, skin, and respiratory irritant, and inhalation of even low levels of $\mathrm{CR}$ has been reported to cause extreme respiratory irritation (157). The United States Environmental Protection Agency further classifies CR as a possible human carcinogen (162). Additionally, CR has been linked to the production of oxidative stress $(163,164)$, the dysfunction of cardiomyocyte contractility (164), and the development of atherosclerosis in African-American men occupationally exposed to 1,3butadiene, the parent compound of CR $(165,166)$. Similarly, inhalation of 1,3-butadiene was shown to accelerate the development of arteriosclerotic lesions in cockerels (167). However, despite these findings and despite the physical and chemical similarities between $\mathrm{CR}$ and acrolein, more research is needed to further elucidate the role of $\mathrm{CR}$ in the development of CVD.

\section{Electronic Cigarettes}

A recent report by the $\mathrm{CDC}$ found that smoking rates among American adults were down to $13.7 \%$ in 2018 , an all-time low set by the continuing decline in smoking rates over the past few decades (83). However, a new class of nicotine-delivery devices has recently been introduced, giving traditional tobacco users a new route for their nicotine 
consumption. Electronic nicotine delivery systems (ENDS) deliver an inhalable aerosol created by heating a solution known as an e-liquid. Liquids in ENDS are composed of a humectant such as propylene glycol (PG) and/or vegetable glycerin (VG) and typically contain nicotine (168-171); many users also use flavor additives (96, 172-174). The most commonly used ENDS are electronic cigarettes (e-cigs) (173, 175-177). Although the first patent for a smokeless non-tobacco cigarette was first filed in the United States in 1965 (178), e-cigs did not become widely available until the early 2000s (173). E-cigs are simply designed, consisting of a battery, a cartridge to contain the e-liquid, and a heating element to vaporize the e-liquid and produce the aerosol $(96,173,175,179)$. Different generations of e-cigs vary somewhat in appearance, but these basic elements are all still present (175, 180).

Usage of ENDS devices has increased dramatically over the past several years, with a significant rise in the use of e-cigs. The 2018 National Health Interview Survey estimated that 49.1 million American adults used any tobacco product, with approximately of $3.2 \%$ (8.1 million) of individuals reporting use of a tobacco product other than traditional cigarettes reporting use of e-cigs (83). Among total tobacco product users, $42.6 \%$ reported daily use of e-cigs, and of the 9.3 million current dual-use tobacco product users, more than $30 \%$ report using both traditional cigarettes and e-cigs (83). In another national sampling of adults in the United States, $84.5 \%$ of e-cig users reported usage as a smoking cessation tool or a healthier alternative (181). Because the long-term health effects of e-cigs are still unknown, the increased use of e-cigs among adults may beget worrisome health conditions in the future, but more alarming is the trend in e-cig use among youth. E-cigs have been the most commonly used tobacco product among American middle (grades 6-8) and high 
school (grades 9-12) students since 2014 (182). The 2018 National Youth Tobacco Survey estimated that $20.8 \%$ of high school students and $4.9 \%$ of middle school students were current e-cig users, with current use defined as usage on one or more day(s) during the past month, with exclusive use of e-cigs reported by $42.0 \%$ and $42.7 \%$ of high school and middle school students e-cig users, respectively (182). Further analysis of these data showed that although there was no significant change in use of any tobacco product overall among high school and middle school students during 2011-2018, there were significant nonlinear increases in e-cig use specifically among both age groups (182); a 77.8\% increase among high school students and a 48.5\% increase among middle school students were reported in 2017-2018 alone despite no other significant changes in the use of other tobacco products occurring during this time period (182). The 2019 National Youth Tobacco Survey showed that usage has further increased, with an estimated $27.5 \%$ of high school students and $10.5 \%$ of middle school students reporting current use of e-cigs, with the majority of e-cig only users using flavored e-cigs (183).

As previously mentioned, e-cig use is commonly cited as a means of smoking cessation or as a healthier alternative to traditional tobacco smoking $(173-175,177,181$, 184). This latter claim is largely due to the fact that e-cigs do not contain actual tobacco leaf, nor does their use involve combustion $(175,180)$. However, the true health effects of these devices are currently unknown. Early studies of the health effects of e-cigs have shown increased reports of cracked teeth and tongue and/or inside-cheek pain in adolescent e-cig users (185), increased reports of dry mouth, sore throat, and acute cough in neversmokers and of acute cough in smokers with and without a history of respiratory disease (186), significantly increased airway resistance $(187,188)$, and impaired mesenchymal 
stem cell differentiation (189). Studies in humans have also shown increases in markers of oxidative stress after vaping $(190,191)$, and levels of ROS have been measured in e-cig aerosol (192).

In mice, a two week exposure to e-cigs significantly increased levels of oxidative stress and impaired pulmonary anti-microbial defenses, leading to enhanced influenza Ainduced illness and mortality (174). A similarly impaired pulmonary response to an infectious challenge was seen in mice exposed for four months to 60\%:40\% PG:VG alone (193). Other studies in mice link chronic e-cig exposure with aortic stiffness and impaired aortic endothelial function in female C57BL/6 mice (194) and to cardiac dysfunction, atherosclerotic development, and increased oxidative stress in apolipoprotein-E knockout mice (195). In rats, acute exposure to e-cig aerosol from either a JUUL device or from an earlier generation e-cig showed impaired endothelium function as measured by flow mediated dilation in both males and females (196). Furthermore, female rats exposed to ecigs during pregnancy show decreased circulation in both the maternal uterine and fetal umbilical cords, indicating potential development of negative health effects in the next generation related to maternal e-cig use while pregnant, an outcome similar to that seen with the use of traditional cigarettes during pregnancy (197).

More studies have focused on the potential toxicity of the components within the aerosol produced by e-cigs. These analyses have revealed that the aerosol, like MCS, contains many harmful or potentially harmful components, including particulates (184, 198-202), metals (184, 203-205), and VOCs, including many aldehydes, (199, 201, 206208), although possibly at lower levels than found in traditional cigarette smoke. Specific aldehydes, including FR, AA, and acrolein, have also been detected in e-cig aerosol (96, 
180, 207-213). PG and VG, whether individually or together, have been shown to be responsible for some of this aldehyde generation (168, 214-216). Generated levels of unsaturated aldehydes, however, are much lower than those found in MCS and much lower compared with generated levels of saturated aldehydes, with CR showing little if any generation in different e-cig flavorings and humectants $(96,168)$.

However, the flavorings themselves could also be contributing to negative health outcomes, a concern on its own but even more so as most youths report using flavored ecigs (183). In 2014, Zhu et al. (217) reported that there were at least 7,764 unique flavors of e-cig liquids sold by 466 distinct e-cig brands. This number has grown as the popularity of e-cigs has increased and as individuals have begun creating and using their own flavors. This wide variety of flavors may pose a health risk that is only beginning to be recognized. Many of the flavorings used to make flavored e-cig liquids come from food flavorings that are deemed by the Flavor and Extract Manufacturers Association as safe for ingestion but not necessarily for inhalation (218). Historically, the inhalation of aerosolized diacetyl, a flavoring that had been evaluated as safe for ingestion, lead to the development of bronchiolitis obliterans in a number of microwave popcorn production plant workers (219), and many in the healthcare industry are fearful of the development of similar pulmonary diseases in response to the use of flavored e-cig liquids (220-223).

These fears seem to be founded, as, over the past year, a number of lung injury cases have been associated with e-cig use (224-230). The CDC reports that as of February 18,2020 , (the date of the last online update) more than 2,800 cases of e-cigarette, or vaping, product use associated lung injury (EVALI) have been reported from all 50 states, the District of Columbia, and 2 U.S. territories, and 68 EVALI deaths have been confirmed 
(230). Of the admitted patients, approximately $76 \%$ are under the age of 35 , with $15 \%$ aged under 18 years old (230), the age group most likely to be using e-cigs based on the appeal of the flavors rather than as a cessation aid $(231,232)$. Vitamin E acetate, a thickening agent used in many tetrahydrocannabinol (THC)-containing e-cig liquids, has been identified as the primary cause of EVALI $(225,230,233)$, and many states have taken steps to prevent further e-cig-related illnesses, especially among youths, by placing temporary statewide bans on all or some flavored e-cigs and other ENDS (234-240).

In terms of cardiovascular outcomes, early studies of flavorings in endothelial cell lines have shown that flavors can pose a cardiovascular health threat through the development of endothelium dysfunction $(241,242)$, but more research is needed to better clarify the vascular toxicities that may be induced by e-cigs. There is also a need to determine how the presence of nicotine in these devices may contribute to adverse health effects. The cardiovascular effects of nicotine have been studied extensively in relation to traditional cigarettes, showing increased heart rate (243-245), blood pressure $(243,244$, 246 , 247), platelet aggregation $(248,249)$, and atherosclerotic development $(250,251)$. However, the amount of nicotine in e-liquids can vary substantially (252-255), which could have an impact on the use of e-cig devices as a harm reduction or cessation tool for traditional smokers and on new use from never-smoking individuals, particularly adolescents (256-259)

Regardless, it is clear that e-cigs are contributing to the development of disease in individuals with an otherwise low risk of disease, and although the specific cause of these illnesses has yet to be definitively identified, it is possible that the presence of saturated and unsaturated aldehydes, either alone or in combination with other constituents, in e-cig 
aerosol may be contributing to the growing health threat posed by these devices.

\section{Transient Receptor Potential Ankyrin 1 Channel}

Unsaturated aldehydes such as acrolein have been shown to work through activation of the transient receptor potential ankyrin 1 (TRPA1) channel. The TRPA1 channel is a member of the TRP superfamily of cation channels and the only member of the ankyrin subfamily (260). TRPA1 is a non-selective cation (calcium) channel and promiscuous sensory receptor concentrated in sensory fibers that mediates pain reception/transmission as well as irritant-related pulmonary reflexes including cough, respiratory braking (slowing rate and depth of breathing), and bronchoconstriction (261). TRPA1 has been shown to be activated by a number of stimuli, including temperature (260, 262), pressure (262), and ROS (263-266), as well as by noxious compounds including diesel exhaust (267), cigarette smoke (261), acrolein (260, 262, 268, 269), cinnamaldehyde $(260,262,266,268,270)$, and FR (127). The activation of the channel by the unsaturated aldehydes or compounds containing these aldehydes is proposed to occur through aldehyde conjugation with the TRPA1 N-terminal free cysteines (271).

Although TRPA1 was initially identified in sensory neurons (272), the channel has since been found in non-neuronal locations, including the endothelium $(260,263,270$, $272)$, urothelium $(273,274)$, vasculature $(275,276)$, and heart $(277,278)$. TRPA1 is known to primarily play a role in inflammatory and pain responses, likely through the release of vasoactive peptides such as substance $\mathrm{P}$ and calcitonin gene-related peptide that increase blood flow, vascular permeability, and leukocyte binding - a collective process referred to as 'neurogenic inflammation' $(262,272,279-285)$. TRPA1 is also important in respiratory irritant responses $(262,279,280,286-288)$, and some studies have shown that 
the channel helps mediate vasodilation though calcium influx $(127,265,266,270,272$, 275). Despite data showing that the negative cardiovascular effects of inhaled acrolein are TRPA1-dependent $(261,269,289,290)$, the role of TRPA1 in potential cardiovascular toxicity of other aldehydes and mixtures (e-cig aerosol) is not well known.

\section{Statement of Goals}

Early studies of e-cig aerosol exposure have shown the development of endothelium dysfunction similar to that seen with tobacco smoke. Many of these studies hypothesize that this dysfunction is related to shared constituents between MCS and e-cig aerosols, including acrolein, FR, and AA. Although previous research has linked acrolein with cardiovascular toxicity in both humans and mice, the effects of other aldehydes on endothelium function and repair are unknown. Therefore, the objective of this project is to evaluate the mechanism of tobacco product-induced CVD via the development of endothelium-related changes, as well as systemic and hematological effects.

\section{B. AIMS AND PROPOSALS}

\section{Measure hematological and endothelium-related changes in humans in response to tobacco-product related pollutant exposures.}

Both ambient and personal air pollutant exposures have been linked to decreased CAC levels in humans, but the health outcomes vary with different forms and durations of exposure. Thus, we will examine the response of CACs and other biomarkers of hematological change to personal exposures (tobacco use, e-cig use, dual product use) in a low to medium-risk heart disease human cohort. Measures of urinary metabolites will also be measured to determine whether VOCs found in MCS are generated in similar levels in 
e-cigs and to establish the potential cardiovascular health risk of these compounds.

\section{Measure systemic, hematological, and endothelium-related changes in mice in response to tobacco products and constituent aldehydes.}

Exposures to both tobacco smoke and e-cig aerosol have been linked to the development of endothelium dysfunction and downstream CVD. Using C57BL/6J (wild type, WT) male mice, we will measure concentration- and duration-dependent changes in CACs and other hematological biomarkers as well as markers of systemic change following exposure to MCS, e-cig aerosol, and aldehydes (FR, AA, acrolein, and CR) found in tobacco smoke and/or e-cig aerosol as well as humectants (PG:VG) used in e-cig devices. We will also exposure female WT mice to determine whether exposure-induced effects are sex-dependent.

\section{Test the roles of ecSOD and TRPA1 in the development of hematological, systemic, and endothelium-related changes contributing to CVD.}

The use of ecSOD-transgenic mice has been shown to reduce levels of ROS in response to ambient exposures, but these mice have not been extensively used to relate exposure to downstream vascular injury and disease. Likewise, mice lacking expression of the TRPA1 gene have been used to examine cardiopulmonary toxicity in response to direct exposure to unsaturated aldehydes, but these mice have not been extensively used to relate exposure to specific markers of downstream endothelium dysfunction and to changes in hematological and systemic biomarkers. We will thus use extracellular superoxide dismutase transgenic (ecSOD-Tg) mice-mice overexpressing extracellular superoxide dismutase in the lungs - and mice lacking expression of TRPA1 (TRPA1-null mice) to test the roles of ecSOD and TRPA1 in the mechanism of cardiovascular-related outcomes 
resulting from in vivo aldehyde exposures.

\section{Overall Aim of this Dissertation}

This dissertation aims to address the significant and global problem of CVD. In particular, this research seeks to investigate the relationship between CVD and air pollutant exposures. The proposed studies will focus on the role of personal exposures to MCS and e-cig aerosols, as well as shared constituents, on systemic, hematological, and endothelium-related outcomes that have been linked to the development of CVD and will seek to identify the mechanism through which these compounds act. These studies will also work to determine whether these effects differ between male and females. The results of this research will contribute to the growing knowledge of CACs and their role in the development of CVD as well as to the field of environmental cardiology in relation to personal pollutant exposures. Furthermore, the results of this research have the potential to contribute to regulation regarding harmful and potentially harmful constituents of tobacco-based products and their contribution to cardiovascular toxicity.

The focus of this study is the relationships between CACs, systemic and hematological biomarkers, and exposure to pollutants, specifically tobacco-based aerosols and specific aldehydes found in MCS and/or e-cig aerosols. Chapter 3 explores the relationship between CACs and tobacco product-related exposures in a human cohort. Chapter 4 investigates the effects of MCS, e-cig aerosol, and PG:VG on CACs and biomarkers in murine models, while chapters 5 and 6 examine exposure-related changes from saturated and unsaturated aldehyde exposures, respectively. Chapter 7 examines the roles of ecSOD and the TRPA1 channel in the cardiovascular toxicity related to tobacco product use. Together, these data will provide novel insight into the cardiovascular impact 
of traditional and emerging tobacco products and provide new understanding of the relationship between CACs and personal pollutant exposures. 


\section{CHAPTER II}

\section{EXPERIMENTAL PROCEDURES}

\section{A. Human Studies}

\section{Study Populations}

To test the research hypothesis, a cohort of 143 participants with varying types of tobacco exposure was recruited for the Louisville Healthy Heart Study (LHHS) from September 2015 to April 2018. Participants were recruited through locally-placed advertisements, by word of mouth, or from nearby cardiovascular disease (CVD) clinics. Clinic enrollment was specific to adults undergoing primary or secondary preventative treatment for cardiovascular disease at University of Louisville and KentuckyOne Health hospitals clinic systems. Enrollment outside the clinic was focused on individuals currently using tobacco products, with a specific focus on individuals who were e-cigarette (e-cig) only users. All advertisements, study consent forms, and research protocols were approved through the University of Louisville's Institutional Review Board (IRB 09.0174).

\section{Eligibility}

Eligibility was determined based on age $(\geq 18$ years of age at the time of enrollment) and, when available, medical records in the general and preventative cardiology and family medicine clinics of the KentuckyOne Health hospitals located in and 
around the University of Louisville's Health Science Campus in downtown Louisville, KY and from the Department of Public Health \& Wellness screening database (set up previously under the direction of Drs. Troutman and Zahn by the Louisville Metro Health Department). Exclusion criteria included: 1) persons unwilling or unable to provide informed consent; 2) persons with significant and/or severe co-morbidities including the following conditions: significant chronic lung, liver, kidney, hematological, or neoplastic disease; chronic neurological or psychiatric illness; chronic infectious disease such as HIV or hepatitis; severe coagulopathies; drug/substance abuse; and chronic cachexia; 3) pregnant women; and 4) prisoners and other vulnerable populations.

\section{Case Definition}

For this study, primary prevention cases were defined as individuals who have known heart disease risk factors (e.g., hypertension, hypercholesterolemia, obesity, diabetes) but no evident coronary artery disease; secondary prevention individuals were those with evident heart disease in addition to heart disease risk factors that require treatment. Specifically, these latter individuals had a clinical manifestation of coronary artery disease and were being treated for risk factors. Fundamentally, the designation of a patient as a primary or secondary preventative case was based on the absence or presence of coronary artery disease.

\section{Recruitment}

Study participants had no restriction on geographic location of residence. Recruitment took place through local print and electronic advertisements, by word of mouth, and at nearby cardiovascular clinics. If recruitment occurred at a clinic, patients were approached during their clinic visit. Interested individuals were fully informed as to 
the purpose of the study and their eligibility confirmed. Individuals willing to participate were either recruited immediately following their clinic visit or required to schedule a separate appointment at their convenience. At this recruitment appointment, individuals were asked to sign informed consent forms and administered a brief questionnaire to obtain demographic information, exposure history, and baseline clinical characteristics.

\section{Questionnaire}

All study participants were administered a questionnaire during their enrollment. This questionnaire was designed to collect relevant demographic and medical information and has been used in other human studies, both as originally written (71) and as modified (291). If previous medical history was available, the questionnaire was used to either confirm or to add to the clinically-indicated standard medical history, physical examination, and laboratory evaluation of traditional CVD risk factors preformed in the clinic. Additional questions sought information regarding the individual's demographic information, residential address, smoking history and current status, secondhand smoke (SHS) exposure, alcohol consumption, physical activity status, use of CVD medications, and CVD history including heart attack, heart failure, angina, high blood pressure, high cholesterol, diabetes, stroke, peripheral artery disease, aortic aneurism, and bleeding disorders. Affirmative answers for the presence of CVD risk factors were based on a professional diagnosis as reported by the patient. Answers were recorded in an electronic format, with the REDCap system (Research Electronic Data Capture; Vanderbilt University Medical Center, Nashville, TN) serving as an electronic database.

\section{Biological Sample Collection and Processing}

After informed consent was given, biological samples (blood and urine) were 
obtained. A random catch urine sample was collected by the participant in the clinic or in the human studies laboratory when enrollment occurred outside the clinic. The urine samples were separated as follows: four 4.5 aliquots, four $2 \mathrm{~mL}$ aliquots, and two $4.5 \mathrm{~mL}$ aliquots with $45 \mu \mathrm{L}$ butylated hydroxytoluene as a preservation additive. Aliquots were stored at $-80^{\circ} \mathrm{C}$ until the time of testing. A blood sample of approximately $25 \mathrm{~mL}$ was obtained via venipuncture by a licensed nurse or other medically-trained individual using standard procedures and precautions to ensure the safety of the participant and the nurse. If any participant became ill or uncomfortable, sample collection was discontinued until the participant recovered, and if the participant wished to withdraw from the study, they were allowed to do so at any time. Approximately $8 \mathrm{~mL}$ of blood was collected in a Vacutainer ${ }^{\circledR}$ CPT Mononuclear Cell Preparation Tube (Becton Dickinson; Franklin Lakes, $\mathrm{NJ})$ for $\mathrm{CAC}$ analysis, $2.6 \mathrm{~mL}$ in a Vacutainer ${ }^{\circledR} \mathrm{ACD}$ tube for platelet mononuclear cell aggregate analysis, and $6 \mathrm{~mL}$ total into a Vacutainer® Serum Tube, a Vacutainer® K2EDTA Tube, and a Vacutainer® Citrate Tube for analysis of plasma components.

\section{Complete Blood Cell Count (CBC)}

A complete blood cell count $(\mathrm{CBC})$ was performed on a Coulter Ac $\bullet \mathrm{T}$ diff2 Hematology Analyzer (Beckman Coulter; Brea, CA). $18 \mu \mathrm{L}$ of collected blood from the Vacutainer® $\mathrm{K}_{2}$ EDTA tube were used for this analysis.

\section{Circulating Angiogenic Cell (CAC) Quantification}

Circulating angiogenic cell (CAC) populations in blood samples were characterized via fluorescence-activated cell sorting (FACS; FACSDiva v6.0 software; BD Biosciences; San Jose, CA) flow cytometry using established markers: CD34+ (endothelial progenitor), CD45+/- (hematopoietic/non-hematopoietic), CD31+ (endothelial cells), and CD133+ (early 
stem cell). Duda et al. (292) first described this method for defining populations of circulating endothelial and progenitor cells in human peripheral blood; a modified version of this protocol has been used in our laboratory to measure populations of human CACs (71, 73). Within 30 minutes of the blood draw, blood cells were separated in the CPT mononuclear separator tube by centrifugation at $1,600 x \mathrm{~g}$ for 30 minutes at room temperature and then stored in an upright position at room temperature overnight. The following day, the upper layers of the sample were decanted into phosphate-buffered saline (PBS) and centrifuged at 500 $\mathrm{gg}$ for five minutes at room temperature. The pelleted cells were then washed twice with $1 \%$ BSA/PBS buffer and then incubated with human FcR Blocking Reagent (Miltenyi Biotec) and a panel of fluorescently-conjugated antibodies on ice for 30 minutes. Fluorescent antibodies used included PE-labeled anti-CD34 (BD Biosciences), APC-labeled anti-AC133 (Miltenyi Biotec; Bergisch Gladbach, Germany), PE-Cy5.5-labeled anti-CD14 (Abcam; Cambridge, United Kingdom), APC-AlexaFluor 750-labeled anti-CD45 (Invitrogen; Carlsbad, CA), PE-Cy7-labeled anti-CD16 (BD Biosciences), FITC-labeled anti-CD31 (BD Biosciences), anti-CD41a (BD Biosciences), anti-CD235a (BD Biosciences), Pacific Blue (Pacific Blue monoclonal antibody labeling kit; Invitrogen), and a marker for dead cells (LIVE/DEAD fixable dead cell stain; Invitrogen). The cells were then washed again with $1 \%$ bovine serum albumin (BSA)/PBS, centrifuged at 500xg for five minutes at room temperature, and resuspended in $1 \%$ BSA/PBS.

Immediately following resuspension, events were collected by FACS using an LSR II flow cytometer (BD Biosciences) for a period of two minutes. Positive and negative boundaries for gating were determined using unstained controls, and sequential gating was 
performed based on antigenic marker groupings. CAC counts were normalized to sample volume for analysis $(71,73)$.

\section{Platelet Mononuclear Cell Aggregate (PMA) Quantification}

A portion of the blood sample was collected in an ACD tube. One $\mathrm{mL}$ of this sample was fixed with a mixture of PBS and $4 \%$ paraformaldehyde and incubated on ice for 30 minutes. Red blood cells were then lysed with the addition of $24 \mathrm{~mL}$ of water and the sample centrifuged at $400 x g$ for 10 minutes at room temperature. Following centrifugation, the pelleted cells were resuspended in Tyrode's buffer and stored at $4{ }^{\circ} \mathrm{C}$ overnight. The following day, $800 \mu \mathrm{L}$ of the sample was centrifuged at $1,000 x g$ for 5 minutes at room temperature then incubated on ice with BSA/Tyrode's buffer and FcR Blocking Reagent (Miltenyi Biotec) for 10 minutes. A second 30-minute incubation with a panel of fluorescently-conjugated antibodies followed, and finally the cells were centrifuged, washed, and resuspended in BSA/Tyrode's buffer. FACS was used to count platelet mononuclear cell aggregates (PMAs) on an LSR II flow cytometer as events that are both FITC $\left(\mathrm{CD} 41_{+}\right)$and PerCP-Cy5.5 (CD45+) positive, with the final count calculated as the percentage of double-positive cells per total events (71).

\section{Urine Metabolite Quantification}

Collected urine was used to quantify levels of creatinine and volatile organic compounds (VOCs) and tobacco alkaloid metabolites as described below.

\section{a. Creatinine}

Concentrations of creatinine were measured on a Cobas Mira Plus Clinical chemistry Autoanalyzer (Roche; Basel, Switzerland) with Infinity Creatinine Reagent 
(ThermoFisher Scientific; Waltham, MA). Urine was diluted 1:10 with deionized water up to $200 \mu \mathrm{L}$ for testing.

\section{b. Volatile Organic Compounds (VOCs) and Tobacco Alkaloids}

Urinary levels of volatile organic compound (VOC) metabolites, including the urinary metabolites of acrolein (3-hydroxypropylmercapturic acid, 3-HPMA) and crotonaldehyde (CR) (3-hydroxy-1-methylpropylmercapturic acid, HPMMA), and tobacco alkaloids metabolites were measured by ultra-performance liquid chromatography tandem mass spectrometry (UPLC-MS/MS) as previously described with slight modifications (293). Briefly, $50 \mu \mathrm{L}$ of sample urine was combined with $400 \mu \mathrm{L}$ ammonium acetate (15 $\mathrm{mM}, \mathrm{pH}$ 6.8) and $50 \mu \mathrm{L}$ internal standard and applied to an ACQUITY UPLC core system (Acquity UPLC HSS T3 column (150 mm x $2.1 \mathrm{~mm}, 1.8 \mu \mathrm{M})$ ) and a Quattro Premier XE triple quadrupole MS coupled with an electrospray source (Waters Corporation; Milford, MA). Ammonia acetate and acetonitrile were used as mobile phase for chromatographic separation. The peak areas of analytes were quantified using at least 12 data points across the measurement peaks, and TargetLynx 4.1 (Waters Corporation) was used for peak integration, calibration, and quantification of analyze concentrations. All values were normalized to urinary creatinine $(\mathrm{mg} / \mathrm{dL})$.

\section{Statistical Analyses}

Descriptive statistics were computed to describe the LHHS cohort and are presented as mean \pm SE for continuous variables and as $\mathrm{n}(\%)$ for categorical variables. Population demographics, CVD history, medication use, markers of systemic injury, markers of thrombosis, and urinary VOC and tobacco alkaloid metabolites were compared across the tobacco product use categories based on self-reported product use and confirmed by 
urinary cotinine levels. The tobacco product use categories used were never smokers, former smokers, sole cigarette users (CIG only), and sole e-cig users or dual users (E-cig or Dual users). Demographic characteristics were compared using one-way analysis of variance (ANOVA) with the Bonferroni post-hoc test for continuous variables and using $\chi$-squared analysis with Fisher's Exact Test for categorical variables. Two participants were excluded from all analyses for misclassification based on inconsistencies between self-report of tobacco use and urinary cotinine levels.

Differences in $\mathrm{CBC}$ results between tobacco product use categories were tested using one-way ANOVA with the Bonferroni post-hoc test. Differences in levels of urinary VOC and tobacco alkaloid metabolites between tobacco product use categories were tested on log-transformed urinary VOC data using one-way ANOVA with the Bonferroni posthoc test.

Relationships in the levels of CACs between tobacco product use categories were assessed on log-transformed CAC measures using multivariable-adjusted linear regression models with never smokers as the reference group. These models utilized the normal probability distribution and the identity link function. Models were initially adjusted for variables found to be potential confounders from the demographic analysis; these variables were age, sex, CVD history, use of CVD medications, family history of CVD, and SHS exposure. Models were also initially adjusted for socioeconomic status (SES) using annual household income as a proxy variable as previously reported $(294,295)$. A random forest model was subsequently used to determine which of the variables used in the fully adjusted model were most likely to have an effect on CAC levels independent of tobacco product use categories. The resulting relative feature importance plot indicated age and SES as 
potential confounders, and a second model examining the relationship between CACs and tobacco product use categories was adjusted only for these two variables.

Analyses of demographic, $\mathrm{CBC}$, and urinary VOC and tobacco alkaloid metabolite data by tobacco product use categories was performed using IBM SPSS Statistics V26 (IBM; Armonk, NY). Multivariable-adjusted linear regression modeling and random forest modeling were performed using $\mathrm{R} \mathrm{V3.6.1} \mathrm{(The} \mathrm{R} \mathrm{Foundation} \mathrm{for} \mathrm{Statistical}$ Computing; Vienna, Austria) and utilizing the following packages: tidyverse V1.3.0 (296), forcats V0.4.0 (297), and randomForest V4.6.14 (298). Statistical significance assumed at $\mathrm{p}<0.05$. Data are expressed as indicated.

\section{B. Murine Studies}

\section{Animals and Treatment}

Male and female C57BL/6J (wild type, WT) mice were obtained from Jackson Laboratories (Bar Harbor, ME). Male extracellular superoxide dismutase transgenic (ecSOD-Tg) mice (on a WT background) mice and male transient receptor potential ankyrin 1 (TRPA1)-null mice (on a WT background) were obtained from breeding colonies at the University of Louisville (Louisville, KY) $(269,299)$. All mice were treated according to the Guiding Principles for the Care and Use of Animals in Research and Teaching as adopted by the American Physiological Society, and all protocols were approved by the University of Louisville Institutional Animal Care and Use Committee (IACUC 16624). Before and during the exposures, mice were housed in the University of Louisville vivarium under pathogen-free conditions, controlled temperatures, and a 12h:12h light:dark cycle. Mice were maintained on a standard chow diet (Rodent Diet 5010, 4.5\% fat by weight, LabDiet; St. Louis, MO). 


\section{Exposures}

\section{a. Mainstream Cigarette Smoke (MCS)}

Male WT, male ecSOD-Tg, and male TRPA1-null mice were exposed to highefficiency particulate air- (HEPA) and charcoal-filtered air (control) or mainstream cigarette smoke (MCS) for 4 days (6h/day) and euthanized immediately after the last exposure. MCS was generated in a state-of-the-art, 4-chamber inExpose system with a software-controlled cigarette smoking robot (Flexiware Software, SCI-REQ; Montreal, Canada) housed in the University of Louisville Inhalation Facility with a separate HVAC system to maintain barrier, temperature and humidity conditions. To generate MCS, a single reference cigarette (3R4F; University of Kentucky, Center for Tobacco Reference Products) was burned at a time (9 puffs, $35 \mathrm{ml} /$ puff, $2 \mathrm{~s} / \mathrm{puff} ; 9 \mathrm{~min} /$ cigarette, 1 cigarette every 30 minutes) for a total of 12 cigarettes burned during the exposure period. Humidified cigarettes (60\% 2-way humidity packet) (Boveda, Inc.; Wayzata, MN) were reloaded between each $2 \mathrm{~h}$ session. The exposure chamber $(5 \mathrm{~L})$ had continuous air flow (3 LPM), and a MicroDust Pro (Casella; Bedford, United Kingdom) continuously measured smoke total suspended particulate (TSP) upstream of chamber. Post-chamber thimble filter collection provided gravimetric TSP data. Mice did not have access to food or water during the exposure.

\section{b. Electronic Cigarettes (E-cigs)}

Male and female WT mice were exposed to either HEPA- and charcoal-filtered air (control) or e-cigarette (e-cig) aerosol for a single $6 \mathrm{~h}$ period or 4 days $(6 \mathrm{~h} / \mathrm{day})$ and euthanized immediately after the last exposure. A custom e-cig holder (SCI-REQ; Montreal, Canada) connected to a fully programmable, software-controlled (Flexiware 
Software, SCI-REQ) exposure system was used to produce the e-cig aerosol. Blu Plus+ ecigs with Classic Tobacco flavoring (BLU Products; Doral, FL) were used for the blu Plus+ exposure, and the accompanying blu Plus+ batteries and cartridges were purchased locally. JUUL e-cig liquids with Virginia (VA) Tobacco, Mango, and Menthol flavorings (Juul Labs, Inc.; San Francisco, CA) with blu Plus+ batteries were used for JUUL exposures.

The blu Plus+ e-cigs exposure was performed under the following conditions: 1) battery charged overnight; 2) new cartridge each day; 3) Classic Tobacco flavor (16 mg nicotine); 4) vaping protocol of 4s puff, 50-60 $\mathrm{mL}$ puff, 2 puffs $/ \mathrm{min} \times 9 \mathrm{~min}=18$ puffs $=$ 1 session; 5) each 6 h exposure had 20 sessions ( 1 session $=9$ min vaping followed by 9 min bias air flow); and, 6) blu Plus+ LED lights during each puff (or is rapidly replaced with charged battery).

The JUUL e-cig exposures were performed under the following conditions: 1) battery charged overnight; 2) e-liquids placed in Mistic bridge cartomizer (Mistic Electronic Cigarettes; Charlotte, NC); 3) new cartomizer used each day; 4) VA Tobacco (5\% nicotine), Mango, or Menthol flavor; 5) vaping protocol of 4s puff, $91 \mathrm{~mL}$ puff, 2 puffs $/ \min$ x 9 min $=18$ puffs $=1$ session; 6 ) each $6 \mathrm{~h}$ exposure had 20 sessions $(1$ session = 9 min vaping followed by 9 min bias air flow); and, 7) bias air flow continued for at least 15 min post-exposure to clear chamber.

Real-time monitoring (computer data logging) of TSP was done with an inline (prechamber) Microdust Pro near infrared detector (Casella; Bedford, United Kingdom), and humidity equilibrated gravimetric filters (post-chamber) were used throughout the exposure. Mice did not have access to food or water during the exposure.

c. Propylene Glycol and Vegetable Glycerin (PG:VG) 
Male and female WT mice were exposed to either HEPA- and charcoal-filtered air (control) or $50 \% / 50 \%$ or $30 \% / 70 \%$ propylene glycol:vegetable glycerin (PG: $\mathrm{VG})$ for a single $6 \mathrm{~h}$ period or 4 days (6h/day). Aerosol from the PG:VG mixtures were generated using a refillable, clear tank (approximately $5 \mathrm{~mL}$ ) atomizer with a coil resistance of 1.8 Ohm (Mistic Electronic Cigarettes; Charlotte, NC) coupled with a rechargeable battery from a blu Plus+ e-cig $(3.7 \mathrm{~V})$. The atomizer tank was weighed before and after the exposure session to determine solution consumption. The vaping protocol of the aerosolized 50\%/50\% PG:VG consisted of a 4s puff, 50-60 $\mathrm{ml}$ puff, 2 puffs $/ \mathrm{min} \times 9 \mathrm{~min}=$ 18 puffs $=1$ session; each $6 \mathrm{~h}$ exposure period has 20 sessions $(1$ session $=9$ min vaping followed by 9 min bias air flow). The vaping protocol of the aerosolized 30\%/70\% PG:VG consisted of $4 \mathrm{~s}$ puff, $91 \mathrm{ml}$ puff, 2 puffs $/ \min \times 9 \mathrm{~min}=18$ puffs $=1$ session; each $6 \mathrm{~h}$ exposure period has 20 sessions ( 1 session $=9$ min vaping followed by 9 min bias air flow $)$. Mice did not have access to food or water during the exposures.

\section{d. Formaldehyde (FR)}

Male and female WT mice were exposed to either HEPA- and charcoal-filtered air (control) or formaldehyde (FR) for 4 days ( 2 or 5 ppm, 6h/day) or 2 weeks ( 5 ppm; 6h/day, 7 days/week) using a custom exposure system and certified permeation tubes (Kin-Tek; LaMarque, TX) as previously described (151). FR vapor was diluted with HEPA-filtered room air, and the FR concentration was continuously monitored using an in-line photoionization detector (MultiRae, Rae Industries; Sunnyvale, CA) upstream of a polycarbonate cage (about $31 \mathrm{~L}$ ). Air or FR was delivered through a fine mesh screen at 8-10 L per minute via a custom cyclone-type top that distributed gas within $10 \%$ of the mean concentration at six locations in the cage (Teague Enterprises; Woodland, CA). Mice 
did not have access to food or water during the exposure.

e. Acetaldehyde (AA)

Male and female WT mice were exposed to either HEPA- and charcoal-filtered air (control) or acetaldehyde (AA) for 4 days (5 ppm, 6h/day) or 2 weeks (5 ppm; 6h/day, 7 days/week) and euthanized immediately following exposure on the final day. The experimental protocol for the exposure is the same as described for FR above with the substitution of AA vapor.

\section{f. Acrolein}

Male WT mice were exposed to either HEPA- and charcoal-filtered air (control) or acrolein for 4 days ( 1 ppm, 6h/day) and euthanized immediately following exposure on the final day. The experimental protocol for the exposure is the same as described for FR above with the substitution of acrolein vapor.

\section{g. Crotonaldehyde (CR)}

Male and female WT mice and male TRPA1-null mice were exposed to either HEPA- and charcoal-filtered air (control) or crotonaldehyde (CR) for 4 days (1 or 3 ppm, 6h/day) or 12 weeks ( 1 ppm; 6h/day, 5 days/week) and euthanized immediately following exposure on the final day. The experimental protocol for the exposure is the same as described for FR above with the substitution of CR vapor.

\section{Urine Collection}

For each type of exposure, urine was collected using rodent metabolic chambers (Nalgene, Lab Products Inc.; Seaford, DE). After exposure, mice were placed singly per metabolic cage (Harvard Apparatus; Cambridge, MA) with 3\% glucose/0.125\% saccharin solution drinking water but without food to collect urine (in graduated cylinders in $4{ }^{\circ} \mathrm{C}$ 
water-jacketed organ baths) in $1 \mathrm{~h}$ increments up to $3 \mathrm{~h}$ post-exposure followed by a 3-16h (overnight, $\mathrm{O} / \mathrm{N}$; mice given food) urine collection $(151,300)$. Collected urine samples were centrifuged (1800xg, $5 \mathrm{~min}$; to pellet any feces or food particles) in cold immediately after collection before being decanted and stored at $-80^{\circ} \mathrm{C}$.

\section{Urine Metabolite Quantification}

Collected urine was used to quantify levels of volatile organic compound (VOC) metabolites and metabolites of saturated aldehydes as described below.

\section{a. Formate and Acetate}

Urinary levels of formate and acetate, the metabolites of formaldehyde (FR) and acetaldehyde (AA), respectively, were measured by gas chromatography-mass spectrometry (GC-MS) as adapted and modified from previous reports $(301,302)$. In short, $20 \mu \mathrm{L}$ of collected urine was mixed with $20 \mu \mathrm{L}$ sodium phosphate $(0.5 \mathrm{M}, \mathrm{pH} 8.0)$ containing ${ }_{13} \mathrm{C}$-formate $(2.3 \mathrm{mM})$ and ${ }_{13} \mathrm{C}_{1}$-acetate $(0.23 \mathrm{mM})$ internal standards, and pentafluorobenzyl bromide $(130 \mu \mathrm{L}, 0.1 \mathrm{M})$. The mixture was vortexed for 1 minute and then incubated at $60^{\circ} \mathrm{C}$ for fifteen minutes, and the resulting reaction products were extracted using hexane $(300 \mu \mathrm{L})$ before being transferred to glass tubes for GC-MS analysis. The peak areas of analytes were quantified using at least 12 data points across the measurement peaks, and TargetLynx 4.1 (Waters Corporation) was used for peak integration, calibration, and quantification of analyze concentrations. Measured formate and acetate sample concentrations were corrected for the natural abundance of ${ }_{13} \mathrm{C}$ isotopes.

\section{b. Volatile Organic Compounds (VOCs) and Tobacco Alkaloids}

Urinary levels of volatile organic compound (VOC) metabolites, including the 
urinary metabolites of acrolein (3-HPMA) and crotonaldehyde (CR) (HPMMA), and tobacco alkaloids metabolites were measured by ultra-performance liquid chromatography tandem mass spectrometry (UPLC-MS/MS) as previously described with slight modifications $(293,303)$. Briefly, $10 \mu \mathrm{L}$ of sample urine was combined with $440 \mu \mathrm{L}$ ammonium acetate $(15 \mathrm{mM}, \mathrm{pH} 6.8)$ and $50 \mu \mathrm{L}$ internal standard and filtered through a 0.2 $\mu \mathrm{m}$ PTFE membrane into a $2 \mathrm{~mL}$ UPLC vial. The samples were then applied to an ACQUITY UPLC core system (Acquity UPLC HSS T3 column (150 mm x $2.1 \mathrm{~mm}, 1.8$ $\mu \mathrm{M})$ ) and a Quattro Premier XE triple quadrupole MS coupled with an electrospray source (Waters Corporation). Ammonia acetate $(15 \mathrm{mM}, \mathrm{pH} 6.8)$ and acetonitrile were used as mobile phase for chromatographic separation. The peak areas of analytes were quantified using at least 12 data points across the measurement peaks, and TargetLynx 4.1 (Waters Corporation) was used for peak integration, calibration, and quantification of analyze concentrations.

\section{Tissue Collection and Storage}

Immediately following the final exposure, mice were anesthetized with sodium pentobarbital ( $\approx 150 \mathrm{mg} / \mathrm{kg}$, i.p.) followed by ventral thoracotomy and exsanguination via cardiac puncture for blood collection in EDTA-coated syringes. Whole blood was taken for complete blood cell count (CBC) analysis $(50 \mu \mathrm{L})$ and circulating angiogenic cell (CAC) analysis $(300 \mu \mathrm{L})$. The remaining blood was collected for additional tests or immediately centrifuged ( $4.6 \mathrm{rpm}, 20 \mathrm{~min}, 4^{\circ} \mathrm{C}$ ) to separate plasma; plasma was subsequently stored at $-80^{\circ} \mathrm{C}$ for further analysis. Collected tissues (heart, lung, kidney, liver, pancreas, spleen, fat pad, and/or skeletal muscle) were frozen in liquid nitrogen and stored at $-80^{\circ} \mathrm{C}$ for further analysis. 


\section{Complete Blood Cell Count (CBC)}

Complete blood cell counts (CBCs) were measured (20 $\mu \mathrm{L}$ blood) with a hematology analyzer calibrated with multispecies hematological reference controls (Hemavet 950FS; Drew Scientific, Inc.; Miami Lakes, FL) as described (151).

\section{Plasma Biomarkers Quantification}

Plasma total cholesterol, high-density lipoprotein (HDL), low-density lipoprotein (LDL), triglycerides, albumin, total protein, aspartate transaminase (AST), alanine transaminase (ALT), lactate dehydrogenase $(\mathrm{LDH})$, creatine kinase $(\mathrm{CK})$, and creatinine were measured on a Cobas Mira Plus clinical chemistry Autoanalyzer (Roche Diagnostics; Indianapolis, IN) as previously described (151) or on a Vet Axcel clinical chemistry system (Alfa Wassermann Diagnostic Technologies, LLC; West Caldwell, NJ).

\section{Circulating Angiogenic Cell (CAC) Quantification}

A $300 \mu \mathrm{L}$ sample of whole blood was lysed (3 $\mathrm{mL}$ BD PharmLyse, BD BioSciences, San Jose, CA; $10 \mathrm{~min}, \mathrm{RT}$ ), and after centrifugation (5 min, 500xg, RT), the supernatant was aspirated. The cell pellet was vortexed and resuspended in $1 \%$ BSA/PBS and divided into 2 equal fractions (i.e., isotype control or Flk-1/Sca-1 labeling). After another centrifugation (5 $\mathrm{min}, 500 x \mathrm{~g}, \mathrm{RT}$ ), mononuclear cells were re-suspended in $1 \%$ BSA/PBS with $5 \mu \mathrm{L}$ anti-mouse CD16/CD32 Fc Block (0.5 $\mu \mathrm{g}$; BD Biosciences), and the samples incubated for on ice. After 10 minutes, $5 \mu \mathrm{L}$ of staining cocktail containing antiSca-1 (FITC; BD BioSciences) and anti-Flk-1 (APC, BD BioSciences) antibodies was added to each sample and the samples incubated on ice for 30 minutes. Cells were then washed with $1 \%$ BSA/PBS and centrifuged (5 min, 500xg, RT). After being resuspended 
in $250 \mu \mathrm{L}$ of $1 \% \mathrm{BSA} / \mathrm{PBS}, 5 \mu \mathrm{L}$ of propidium iodide stock staining solution (ThermoFisher Scientific) was added to each tube, and cells were analyzed using a LSRII flow cytometer (BD BioSciences). Based on forward and side scatter, small non-debris events in a sub-lymphocyte population (3-5 $\mu \mathrm{m}$; sized using calibrated beads, Invitrogen, Carlsbad, CA, USA) were gated electronically and displayed in a two-color dot plot. Data were analyzed using FlowJo software (FlowJo LLC; Ashland, OR), and double positive events were normalized per $1 \mu \mathrm{l}$ of whole blood.

\section{Leukocyte Subpopulation Quantification}

A $100 \mu \mathrm{L}$ sample of whole blood was lysed $(1 \mathrm{~mL}$ BD PharmLyse, BD BioSciences, San Jose, CA; $10 \mathrm{~min}, \mathrm{RT}$ ) and after centrifugation (5 min, 500xg, RT), the supernatant was aspirated. The cell pellet was vortexed and resuspended with $1 \mathrm{~mL} 1 \%$ BSA/PBS and again centrifuged (5 min, 500xg, RT), aspirated, and vortexed. This process was repeated a third time before the addition of $5 \mu \mathrm{L}$ anti-mouse CD16/CD32 Fc Block (50 $\mu \mathrm{g} / \mathrm{mL}$; BD Biosciences) and incubation on ice. After 10 minutes, the cells were incubated on ice for 30 minutes with a cocktail containing FITC-anti-Nk1.1 (0.5 $\mu \mathrm{g})$, PEanti-Ly6C (0.125 $\mu \mathrm{g})$, PerCPe710-anti-CD8 (0.125 $\mu \mathrm{g})$, PECy7-anti-CD62 (0.25 $\mu \mathrm{g})$, APC-anti-CD19 (0.125 $\mu \mathrm{g})$, Alexa 700-antiGr-1 (0.125 $\mu \mathrm{g})$, APCe780-anti-CD3 (1 $\mu \mathrm{g})$, eVolve605-CD11b (1 $\mu \mathrm{g})$, and e650-anti-CD4 (1 $\mu \mathrm{g})$. The stained cells were once again centrifuged (5 min, 500xg, RT), aspirated, and vortexed before resuspension in $250 \mu \mathrm{L} 1 \%$ BSA/PBS before analysis on an LSR II flow cytometer (high speed, 90 seconds) (BD BioSciences). Cell numbers were analyzed using the FlowJo software (FlowJo LLC) and normalized per $1 \mu \mathrm{l}$ of whole blood. Monocytes double positive for Ly6C and CD62L were defined as a Ly6Chigh subpopulation. 


\section{Statistical Analyses}

Statistical analyses of flow cytometry data were performed using FlowJo V10 (FlowJo LLC; Ashland, OR) and SigmaPlot 12.5 (Systat Software, Inc.; San Jose, CA). Analyses of changes in body and tissue parameters as well as analyses of the results of CBC, plasma biomarker, and urine metabolite testing were performed using Excel 2016 (Microsoft; Redmond, WA) and SigmaPlot 12.5 (Systat Software, Inc.). Rank sum tests with Bonferroni's post-test were used to determine statistical significance when comparing two groups. Two-way ANOVA with Dunn's post-test was used when comparing multiple groups. Statistical significance assumed at $\mathrm{p}<0.05$ or as indicated. Data are expressed as the mean \pm SE or as indicated. 


\section{CHAPTER III}

\section{ASSOCIATIONS BETWEEN CIRCULATING ANGIOGENIC CELLS AND USE \\ OF TRADITIONAL AND EMERGING TOBACCO PRODUCTS}

\section{A. Introduction}

According to the World Health Organization, ischemic heart disease and stroke have been the leading causes of death worldwide since 2000 , accounting for almost $27 \%$ of reported global deaths (2). In the United States, cardiovascular disease (CVD) constitutes a significant health and economic burden. The American Heart Association estimates that by 2035 , more than $45 \%$ of the adult US population will have some form of CVD, and costs for medical care related to CVD will reach almost $\$ 1$ trillion (304). The development of CVD has been linked to the development of endothelium dysfunction; endothelium function is a balance of injury and repair, in part attributed to circulating angiogenic cells (CACs), wherein CAC levels are associated with endothelium health (6266). Many studies have linked decreased levels of CACs with well-known CVD risk factors, including hypertension $(70,305)$, total (66) and low-density lipoprotein (LDL) (70, 306) cholesterol, diabetes (307, 308), flow-mediated brachial reactivity (66), and an increased Framingham Risk Score $(66,309,310)$. Furthermore, levels of CACs have been shown to be decreased in individuals with more severe CVD (311-314), and Hill et al. (66) 
have proposed that CACs may serve as a surrogate biomarker for vascular health and overall CVD risk.

Smoking has also been associated with CACs $(315,316)$. Smoking reduction and cessation have been linked to increased endothelium function attributed to greater levels or function of CACs (317-319), and specific components of traditional cigarettes and other tobacco products have been directly shown to alter levels of CACs $(151,320,321)$. Although the effects of electronic cigarettes (e-cigs) on CACs are still debated (322-324), the many shared components between mainstream cigarette smoke (MCS) and e-cigs may contribute to changes in the number and function of CACs in a way that is likely to contribute to CVD development. Thus, the goal of this study was to examine the effects of personal exposures, specifically exposures to MCS and/or secondhand cigarette smoke (SHS) and e-cig aerosol, on hematological and endothelium-related changes in a human cohort.

\section{B. Experimental Procedures}

\section{Study Population}

Participants were recruited for the Louisville Healthy Heart Study (LHHS) as described in Chapter II, Section A.1.

\section{Eligibility}

Interested individuals were questioned regarding the eligibility requirements outlined in Chapter II, Section A.2. Individuals who met the eligibility requirements were enrolled in the study.

\section{Case Definition}

Study participants who were enrolled through the University of Louisville and 
KentuckyOne Health hospitals cardiovascular clinic systems were defined as undergoing primary or secondary treatment based on the criteria outlined in Chapter II, Section A.3.

\section{Recruitment}

Details of participant recruitment are given in Chapter II, Section A.4.

\section{Questionnaire}

Each participant was administered a questionnaire as described in Chapter II, Section A.5. The questionnaire was designed to collect relevant information regarding the participant's demographics, medical history, and product use.

\section{Biological Sample Collection and Processing}

Upon confirming eligibility and obtaining written consent, blood and urine samples were collected, aliquoted, and stored as described in Chapter II, Section A.6.

\section{Complete Blood Cell Count (CBC)}

A sample of collected blood from the Vacutainer ${ }^{\circledR} \mathrm{K}_{2}$ EDTA tube was used for a complete blood cell count (CBC) as described in Chapter II, Section A.7.

\section{Circulating Angiogenic Cell (CAC) Quantification}

Florescence-activated cell sorting (FACS) flow cytometry was used to analyze the number of CACs in blood samples collected in the Vacutainer® CPT Mononuclear Cell Preparation Tube as described in Chapter II, Section A.8. The gating scheme for this analysis is shown in Figure 3.1. The 15 CAC populations are described in Table 3.1.

\section{Peripheral Blood Mononuclear Cell Aggregate (PMA) Quantification}

FACS flow cytometry was used to analyze the number of platelet mononuclear cell aggregates (PMAs) in blood samples collected in the Vacutainer ${ }^{\circledR}$ ACD tube as described in Chapter II, Section A.9. 


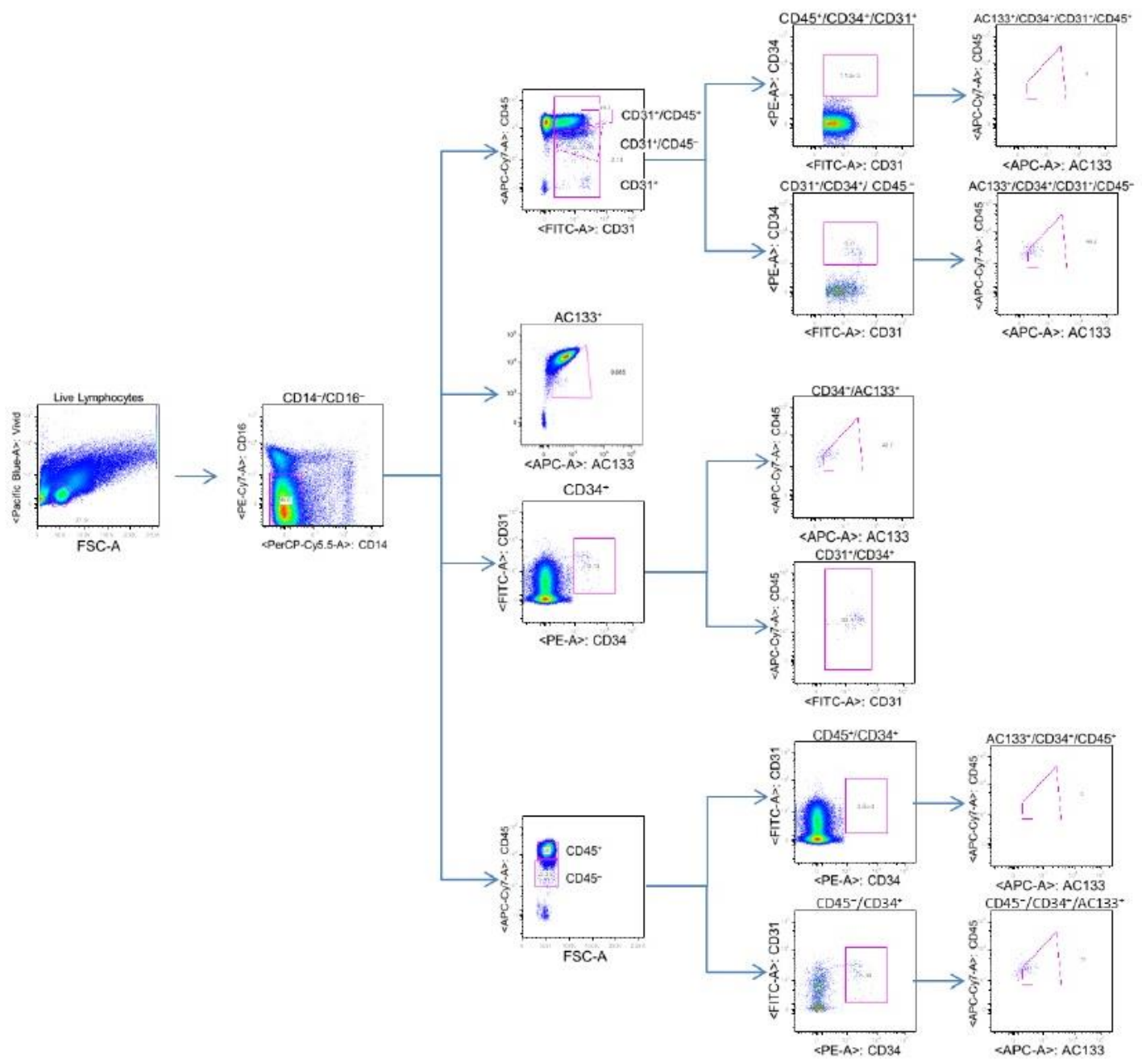

Fig. 3.1. Flow cytometry gating scheme for circulating angiogenic cell (CAC) populations. Abbr.: FSC, forward scatter. 
Table 3.1. Antigenic identity of circulating angiogenic cells (CACs).

\begin{tabular}{|c|c|c|c|}
\hline \multicolumn{2}{|c|}{ Cell Group } & Antigenic Marker & Cell Differentiation State \\
\hline \multirow{3}{*}{$\frac{\Xi}{\text { D }}$} & CAC-9 & CD34+ & Stem \\
\hline & CAC-13 & CD34+/ACC133+ & Stem/Early Progenitor \\
\hline & $\mathrm{CAC}-15$ & $\begin{array}{l}\text { CD34+/ACC133+/ } \\
\text { CD45dim }\end{array}$ & $\begin{array}{l}\text { Stem/Early Progenitor/ } \\
\text { Non-hematopoietic }\end{array}$ \\
\hline \multirow{4}{*}{ 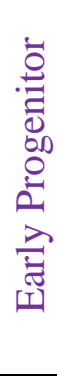 } & CAC-11 & AC133+ & Early Progenitor \\
\hline & CAC-5 & $\mathrm{AC} 133+/ \mathrm{CD} 31+$ & Early Progenitor/Endothelial \\
\hline & CAC-4 & $\begin{array}{l}\mathrm{AC} 133+/ \mathrm{CD} 34+/ \\
\mathrm{CD} 31_{+} / \mathrm{CD} 45_{+}\end{array}$ & $\begin{array}{l}\text { Early Progenitor/Stem/ } \\
\text { Endothelial/Hematopoietic }\end{array}$ \\
\hline & CAC-14 & $\begin{array}{l}\mathrm{AC} 133+/ \mathrm{CD} 34+/ \\
\mathrm{CD} 45+\end{array}$ & $\begin{array}{l}\text { Early } \\
\text { Progenitor/Stem/Hematopoietic }\end{array}$ \\
\hline \multirow{5}{*}{ 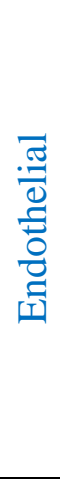 } & CAC-10 & CD31+ & Endothelial \\
\hline & CAC-6 & CD31+/CD34+ & Endothelial/Stem \\
\hline & CAC-1 & $\begin{array}{l}\text { CD31+/CD34+/ } \\
\text { CD45dim }\end{array}$ & $\begin{array}{l}\text { Endothelial/Stem/ } \\
\text { Non-hematopoietic }\end{array}$ \\
\hline & CAC-7 & $\begin{array}{l}\text { CD31+/CD34+/ } \\
\text { AC133-/CD45dim }\end{array}$ & $\begin{array}{l}\text { Endothelial/Stem/ } \\
\text { Late Progenitor/Non-hematopoietic }\end{array}$ \\
\hline & CAC -3 & $\begin{array}{l}\text { CD31+/CD34+/ } \\
\text { AC133+/CD45dim }\end{array}$ & $\begin{array}{l}\text { Endothelial/Stem/ } \\
\text { Early Progenitor/Non-hematopoietic }\end{array}$ \\
\hline \multirow{3}{*}{ 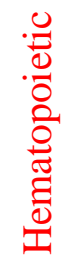 } & $\mathrm{CAC}-12$ & $\mathrm{CD} 45_{+}$ & Hematopoietic \\
\hline & CAC-2 & $\begin{array}{l}\text { CD45+/CD34+/ } \\
\text { CD31+ }\end{array}$ & Hematopoietic/Stem/Endothelial \\
\hline & CAC-8 & $\begin{array}{l}\text { CD45+/CD34+/ } \\
\text { CD31+/AC133- }\end{array}$ & $\begin{array}{l}\text { Hematopoietic/Stem/ } \\
\text { Endothelial/Late Progenitor }\end{array}$ \\
\hline
\end{tabular}




\section{Urine Metabolite Quantification}

Collected random catch urine samples were used to quantify levels of creatinine and volatile organic compound (VOC) and tobacco alkaloids metabolites using analytical methods as described in Chapter II, Section A.10.

\section{Statistical Analyses}

Differences between demographics, measures of systemic injury, urinary metabolites, and CACs were assessed as described in Chapter II, Section A.11.

\section{Results}

\section{Baseline Characteristics by Tobacco Product Use Categories}

Overall, the mean (SE) age of the Louisville Healthy Heart study population was $49( \pm 1)$ years, and the majority of participants were women $(66 \%)$ (Table 3.2). E-cig or dual users were significantly younger than never or former smokers and cigarette only smokers (Table 3.2). There were not notable sex differences, with similar levels of cigarette only and e-cig or dual use between males and females, and both cigarette only and e-cig or dual users were predominately white (68\% and $71 \%$, respectively), although these differences were not statistically significant (Table 3.2). Current nonusers of tobacco were more educated and had higher annual income than current users of tobacco products (Table 3.2). Never smokers reported higher prevalence of high blood cholesterol and hypertension in their family history, while former smokers reported a lower family prevalence of diabetes (Table 3.2). Current tobacco product users reported more secondhand smoke exposure, but reports of alcohol consumption and regular exercise were similar between all use groups (Table 3.2). 
Table 3.2. Demographic characteristics by tobacco product use categories.

\begin{tabular}{|c|c|c|c|c|c|c|}
\hline $\begin{array}{c}\text { Continuous Variables - } \\
\text { mean (SE) }\end{array}$ & Total $(n=143)$ & $\begin{array}{c}\text { Never Smokers } \\
(\mathrm{n}=62)\end{array}$ & $\begin{array}{c}\begin{array}{c}\text { Former Smokers } \\
(\mathbf{n}=25)\end{array} \\
\end{array}$ & $\begin{array}{c}\text { CIG Only } \\
(n=28)\end{array}$ & $\begin{array}{c}\begin{array}{c}\text { E-Cig or Dual } \\
(n=28)\end{array} \\
\end{array}$ & p-value \\
\hline BMI & $30(1)$ & $30(1)$ & $30(1)$ & $29(1)$ & $30(1)$ & 0.263 \\
\hline $\begin{array}{l}\text { Sex } \\
\quad \text { Female } \\
\text { Male }\end{array}$ & $\begin{array}{l}95(66) \\
48(34)\end{array}$ & $\begin{array}{l}47(76) \\
15(24)\end{array}$ & $\begin{array}{l}18(72) \\
7(28)\end{array}$ & $\begin{array}{l}14(50) \\
14(50)\end{array}$ & $\begin{array}{l}16(57) \\
12(43)\end{array}$ & 0.065 \\
\hline $\begin{array}{l}\text { Family Medical History } \\
\text { Diabetes } \\
\text { High Blood Cholesterol } \\
\text { Hypertension } \\
\text { Premature CVD } \\
\end{array}$ & $\begin{array}{l}114(80) \\
66(46) \\
50(35) \\
96(67) \\
38(27) \\
\end{array}$ & $\begin{array}{l}54(87) \\
33(53) \\
29(47) \\
47(76) \\
13(21) \\
\end{array}$ & $\begin{array}{l}17(68) \\
6(24) \\
8(32) \\
17(68) \\
4(16) \\
\end{array}$ & $\begin{array}{l}23(82) \\
12(43) \\
9(32) \\
18(64) \\
11(39) \\
\end{array}$ & $\begin{array}{l}20(71) \\
15(54) \\
4(14) \\
14(50) \\
10(36) \\
\end{array}$ & $\begin{array}{l}0.126 \\
\mathbf{0 . 0 1 4} \\
\mathbf{0 . 0 2 5} \\
\mathbf{0 . 0 1 5} \\
\mathbf{0 . 0 2 8} \\
\end{array}$ \\
\hline $\begin{array}{l}\text { Education } \\
\text { < HS } \\
\text { Some HS/HS Grad } \\
\text { Some College } \\
\text { 2-4 Year Degree } \\
\text { Masters/Doctorate } \\
\end{array}$ & $\begin{array}{l}9(6) \\
22(15) \\
44(31) \\
50(35) \\
17(12) \\
\end{array}$ & $\begin{array}{l}2(3) \\
6(10) \\
10(16) \\
30(49) \\
13(21)\end{array}$ & $\begin{array}{l}3(12) \\
4(16) \\
5(20) \\
11(44) \\
2(8) \\
\end{array}$ & $\begin{array}{l}3(11) \\
5(18) \\
15(54) \\
4(14) \\
1(4) \\
\end{array}$ & $\begin{array}{l}1(4) \\
7(25) \\
14(50) \\
5(18) \\
1(4) \\
\end{array}$ & $<0.001$ \\
\hline
\end{tabular}




\begin{tabular}{|c|l|l|l|l|l|l|}
\hline $\begin{array}{c}\text { Annual Household Income } \\
<\$ 20,000\end{array}$ & $18(13)$ & $5(8)$ & $2(8)$ & $5(18)$ & $6(21)$ \\
$\$ 20,000-\$ 45,000$ & $37(26)$ & $10(16)$ & $5(20)$ & $11(39)$ & $11(39)$ \\
$\$ 45,000-\$ 89,999$ & $47(33)$ & $21(34)$ & $11(44)$ & $8(29)$ & $7(25)$ \\
$>\$ 90,000$ & $41(29)$ & $26(42)$ & $7(28)$ & $4(14)$ & $4(14)$ \\
\hline
\end{tabular}

Values = mean \pm SE. Abbr.: BMI, body mass index; CIG, cigarette; CVD, cardiovascular disease; E-cig, electronic cigarette; HS, high school. 
Two-thirds of participants $(\mathrm{n}=94,66 \%)$ reported some form of CVD history, with almost half of those individuals reporting a diagnosis of hypertension $(n=69,49 \%)$ and approximately one-third reporting a diagnosis of high blood cholesterol $(n=47,33 \%)$ (Table 3.3). Additionally, over half of the participants reported taking some form of CVD medication $(\mathrm{n}=76,53 \%)$ (Table 3.3). By tobacco product use category, fewer e-cig or dual users reported significant CVD history than both never and former smokers as well as less use of CVD medication than all other use categories (Table 3.3). E-cig or dual users were also less likely to have high blood cholesterol and hypertension than all other use categories and to use aspirin or beta-blockers than former smokers, while never smokers were less likely to have hypertension and to use beta blockers than former smokers (Table 3.3). Former smokers and cigarette only users showed similar likelihoods of having some form of CVD and using CVD medication (Table 3.3).

\section{Tobacco Product Use Categories and Hematological Measures}

E-cig or dual users had significantly higher levels of leukocytes than never smokers, driven by a significant increase in granulocytes, as well as an increased level of hemoglobin (Table 3.4). Cigarette only users had an increased red cell distribution width compared with both never smokers and e-cig or dual users (Table 3.4). Although platelets levels (measured by $\mathrm{CBC}$ ) were unchanged between the groups, platelet monocyte aggregates (PMAs; measured by flow cytometry) were significantly different between the categories, with e-cig or dual users showing significantly lower levels of PMAs compared with never smokers, former smokers, and cigarette only users (Table 3.4). Never smokers and former smokers showed no significant differences between any of the hematological measures measured by CBC (Table 3.4). 
Table 3.3. Medical history and medication use by tobacco product use categories.

\begin{tabular}{|c|c|c|c|c|c|c|}
\hline Categorical Variables - n (\%) & $\begin{array}{c}\text { Total } \\
(n=143)\end{array}$ & $\begin{array}{c}\text { Never } \\
\text { Smokers } \\
(n=62)\end{array}$ & $\begin{array}{c}\text { Former } \\
\text { Smokers } \\
(\mathrm{n}=\mathbf{2 5}) \\
\end{array}$ & $\begin{array}{c}\text { CIG Only } \\
(\mathbf{n}=28)\end{array}$ & $\begin{array}{c}\text { E-Cig } \\
\text { or Dual } \\
(n=28)\end{array}$ & p-value \\
\hline \multicolumn{7}{|l|}{ Medical History } \\
\hline CVD History & $94(66)$ & $44(71)$ & $21(84)$ & $18(64)$ & $11(39)$ & 0.005 \\
\hline Angina & $13(9)$ & $4(7)$ & $5(20)$ & $4(14)$ & $0(0)$ & 0.055 \\
\hline Atrial Fibrillation/Flutter & $8(6)$ & $4(7)$ & $2(8)$ & $2(7)$ & $0(0)$ & 0.793 \\
\hline Blood Clot & $7(5)$ & $2(3)$ & $3(12)$ & $2(7)$ & $0(0)$ & 0.135 \\
\hline CABG/PCI/Stents & $15(11)$ & $6(10)$ & $3(12)$ & $4(14)$ & $2(7)$ & 0.651 \\
\hline Heart Failure & $9(6)$ & $1(2)$ & $4(16)$ & $4(15)$ & $0(0)$ & 0.004 \\
\hline Heart Valve Disease & $5(4)$ & $2(3)$ & $2(8)$ & $1(4)$ & $0(0)$ & 0.508 \\
\hline High Blood Cholesterol & $47(33)$ & $22(36)$ & $12(48)$ & $9(32)$ & $4(14)$ & 0.034 \\
\hline Hypertension & $69(49)$ & $31(50)$ & $20(80)$ & $14(52)$ & $4(14)$ & $<0.001$ \\
\hline Heart Attack & $11(8)$ & $4(7)$ & $3(12)$ & $4(14)$ & $0(0)$ & 0.126 \\
\hline Peripheral Artery Disease & $5(4)$ & $3(5)$ & $2(8)$ & $0(0)$ & $0(0)$ & 0.246 \\
\hline Stroke & $7(5)$ & $2(3)$ & $1(4)$ & $2(7)$ & $2(7)$ & 0.809 \\
\hline Other Significant Medical Condition(s) & $39(27)$ & $21(34)$ & $7(28)$ & $4(14)$ & $7(25)$ & 0.288 \\
\hline Diabetes & $28(20)$ & $13(21)$ & $8(32)$ & $4(14)$ & $3(11)$ & 0.234 \\
\hline $\mathrm{BMI} \geq 30$ & $64(45)$ & $26(42)$ & $11(44)$ & $11(39)$ & $16(57)$ & 0.374 \\
\hline \multicolumn{7}{|l|}{ Medication Use } \\
\hline CVD Medication & $76(53)$ & $38(61)$ & $18(72)$ & $15(54)$ & $5(18)$ & $<0.001$ \\
\hline ACE Inhibitor & $26(19)$ & $12(20)$ & $6(24)$ & $7(27)$ & $1(4)$ & 0.122 \\
\hline Angiotensin-receptor Blocker & $12(9)$ & $4(7)$ & $4(16)$ & $4(15)$ & $0(0)$ & 0.147 \\
\hline Aspirin & $33(24)$ & $13(22)$ & $11(44)$ & $8(31)$ & $1(4)$ & 0.006 \\
\hline Beta-blocker & $30(22)$ & $10(17)$ & $12(48)$ & $8(31)$ & $0(0)$ & $<0.001$ \\
\hline Calcium-channel Blocker & $16(12)$ & $7(12)$ & $5(20)$ & $4(15)$ & $0(0)$ & 0.144 \\
\hline Diuretics & $28(21)$ & $11(18)$ & $8(32)$ & $7(27)$ & $2(8)$ & 0.196 \\
\hline Statins & $31(22)$ & $13(21)$ & $9(36)$ & $7(25)$ & $2(7)$ & 0.070 \\
\hline
\end{tabular}




\begin{tabular}{|c|l|l|l|l|l|l|}
\hline Other Medication & $120(84)$ & $54(87)$ & $23(92)$ & $24(86)$ & $19(68)$ & 0.099 \\
Oral Contraceptives & $49(34)$ & $16(26)$ & $7(28)$ & $14(50)$ & $12(43)$ & 0.091 \\
\hline
\end{tabular}

Values = mean \pm SE. Abbr.: ACE, angiotensin-converting enzyme; BMI, body mass index; CABG, coronary artery bypass grafting; CIG, cigarette; CVD, cardiovascular disease; E-cig, electronic cigarette; PCI, percutaneous coronary intervention. 
Table 3.4. Hematological measures by tobacco product use categories.

\begin{tabular}{|c|c|c|c|c|c|c|}
\hline $\begin{array}{c}\text { Complete Blood Cell Count }- \\
\text { mean } \pm \text { SE }\end{array}$ & Total $(n=139)$ & $\begin{array}{c}\text { Never } \\
\text { Smokers } \\
(n=61) \\
\end{array}$ & $\begin{array}{c}\text { Former } \\
\text { Smokers } \\
(\mathbf{n}=\mathbf{2 4}) \\
\end{array}$ & $\begin{array}{l}\text { CIG Only } \\
(\mathbf{n}=27)\end{array}$ & $\begin{array}{l}\text { E-Cig or Dual } \\
\qquad(\mathrm{n}=27)\end{array}$ & p-value \\
\hline White Blood Cell $(\mathrm{K} / \mu \mathrm{L})$ & $6.43 \pm 0.17$ & $5.82 \pm 0.20$ & $6.10 \pm 0.37$ & $7.05 \pm 0.42$ & $7.49 \pm 0.42$ & 0.002 \\
\hline Lymphocytes (\%) & $30.89 \pm 0.62$ & $32.17 \pm 1.02$ & $30.19 \pm 1.10$ & $31.81 \pm 1.52$ & $27.68 \pm 1.19$ & 0.098 \\
\hline Monocytes (\%) & $6.90 \pm 0.16$ & $7.12 \pm 0.25$ & $7.46 \pm 0.33$ & $6.41 \pm 0.35$ & $6.41 \pm 0.42$ & 0.049 \\
\hline Granulocytes (\%) & $62.27 \pm 0.65$ & $60.49 \pm 1.05$ & $63.21 \pm 0.93$ & $61.78 \pm 1.58$ & $65.93 \pm 1.37$ & 0.016 \\
\hline Red Blood Cell (M/ $\mu \mathrm{L})$ & $4.68 \pm 0.05$ & $4.6 \pm 0.06$ & $4.68 \pm 0.12$ & $4.52 \pm 0.09$ & $4.92 \pm 0.14$ & 0.107 \\
\hline Hemoglobin $(\mathrm{g} / \mathrm{dL})$ & $14.1 \pm 0.1$ & $13.8 \pm 0.2$ & $13.9 \pm 0.3$ & $13.9 \pm 0.4$ & $15.0 \pm 0.4$ & 0.029 \\
\hline Hematocrit (\%) & $42.0 \pm 0.5$ & $41.4 \pm 0.5$ & $41.7 \pm 1.0$ & $41.3 \pm 1.2$ & $44.4 \pm 1.3$ & 0.171 \\
\hline Mean Corpuscular Volume (fL) & $89.8 \pm 0.5$ & $89.2 \pm 0.6$ & $89.3 \pm 0.9$ & $91.1 \pm 1.6$ & $90.2 \pm 1.1$ & 0.621 \\
\hline Mean Corpuscular Hemoglobin (pg) & $30.3 \pm 0.3$ & $29.8 \pm 0.3$ & $30.0 \pm 0.5$ & $30.9 \pm 0.8$ & $30.9 \pm 1.0$ & 0.534 \\
\hline $\begin{array}{l}\text { Mean Corpuscular Hemoglobin } \\
\text { Concentration }(\mathrm{g} / \mathrm{dL})\end{array}$ & $33.7 \pm 0.3$ & $33.4 \pm 0.2$ & $33.6 \pm 0.5$ & $34.0 \pm 0.8$ & $34.3 \pm 1.1$ & 0.831 \\
\hline Red Cell Distribution Width (\%) & $14.8 \pm 0.1$ & $14.5 \pm 0.1$ & $15.0 \pm 0.3$ & $15.6 \pm 0.4$ & $14.2 \pm 0.4$ & 0.008 \\
\hline Platelets $(\mathrm{K} / \mu \mathrm{L})$ & $263 \pm 6$ & $256 \pm 8$ & $270 \pm 16$ & $259 \pm 16$ & $276 \pm 13$ & 0.551 \\
\hline PMAs $(\%)$ & $23.3 \pm 0.8$ & $23.9 \pm 1.1$ & $22.8 \pm 1.8$ & $23.7 \pm 2.2$ & $21.6 \pm 1.7$ & $<0.001$ \\
\hline Mean Platelet Volume (fL) & $8.4 \pm 0.1$ & $8.3 \pm 0.2$ & $8.7 \pm 0.4$ & $8.4 \pm 0.3$ & $8.4 \pm 0.2$ & 0.787 \\
\hline
\end{tabular}

Values = mean \pm SE. Abbr.: CIG, cigarette; E-cig, electronic cigarette; PMAs, platelet mononuclear cell aggregates 


\section{Tobacco Products Use Categories and Urinary VOC Metabolites}

Analysis of urinary VOC and tobacco alkaloid metabolites showed that never and former smokers had lower mean levels of most VOC and tobacco alkaloid metabolites compared with current users of tobacco products (Table 3.5). Never smokers and former smokers showed no significantly different levels of measured VOC or tobacco alkaloid metabolites (Table 3.5). All measured metabolites except $\mathrm{N}$-acetyl-S-(benzyl)-L-cysteine (BMA) showed significant differences between the tobacco product use categories, and the majority of VOC metabolites were higher in cigarette only users and/or e-cig or dual users than in never or former smokers (Table 3.5). Almost one-third of the VOC metabolites were significantly different between cigarette only users and e-cig or dual users, with levels of cyanoethyl mercapturic acid (CEMA), 3-hydroxypropylmercapturic acid (3HPMA), Nacetyl-S-(2-carbamoylethyl)-L-cysteine (AAMA), N-acetyl-S-(4-hydroxy-2-buten-1-yl)L-cysteine (3MHBMA), N-acetyl-S-(3-hydroxypropyl-1-methyl)-L-cysteine (HPMMA), $\mathrm{N}$-acetyl-S-(N-methylcarbamoyl)-L-cysteine (AMCC), and anatabine (ANTB) significantly higher in cigarette only users compared with e-cig or dual users (Table 3.5). Interestingly, while never smokers showed significantly lower levels of AMCC, N-acetylS-(2-hydroxypropyl)-L-cysteine (2HPMA), 2-methylhippuric acid (2MHA), and 3methylhippuric acid + 4-methylhippuric acid (3MHA + 4MHA), compared with both cigarette only users and e-cig or dual users, levels of these metabolites in former smokers were not significantly different compared with e-cig or dual users (Table 3.5). Additionally, former smokers had levels of anabasine (ANB) that were not significantly different from either e-cig or dual users or cigarette only users, although levels were also not significantly different from those found in never smokers (Table 3.5). Unsurprisingly, 
Table 3.5. Mean urinary volatile organic compound (VOC) metabolite levels by tobacco product use categories (ng/mg).

\begin{tabular}{|c|c|c|c|c|c|c|c|}
\hline Parent Compound & Analyte & Total $(n=103)$ & $\begin{array}{c}\text { Never } \\
\text { Smokers } \\
(n=43) \\
\end{array}$ & $\begin{array}{c}\text { Former } \\
\text { Smokers } \\
(n=17) \\
\end{array}$ & $\begin{array}{l}\text { CIG Only } \\
(n=23)\end{array}$ & $\begin{array}{l}\text { E-Cig or Dual } \\
\quad(n=20)\end{array}$ & p-value \\
\hline Acrolein & $\begin{array}{l}\text { CEMA } \\
\text { 3НРMA }\end{array}$ & $\begin{array}{l}167.8 \pm 17.3 \\
822.8 \pm 107.6\end{array}$ & $\begin{array}{l}72.0 \pm 14.0 \\
313.8 \pm 67.5\end{array}$ & $\begin{array}{l}71.4 \pm 13.6 \\
267.1 \pm 38.7\end{array}$ & $\begin{array}{l}365.0 \pm 39.7 \\
1,837.9 \pm 280.6\end{array}$ & $\begin{array}{l}228.9 \pm 33.6 \\
1,222.0 \pm 280.7\end{array}$ & $\begin{array}{l}<0.001 \\
<0.001\end{array}$ \\
\hline Acrylamide & AAMA & $103.9 \pm 8.7$ & $67.8 \pm 6.0$ & $69.0 \pm 10.5$ & $192.3 \pm 25.9$ & $109.5 \pm 16.0$ & $<0.001$ \\
\hline 1,3-Butadiene & $\begin{array}{l}\text { DHBMA } \\
\text { 3MHBMA }\end{array}$ & $\begin{array}{l}422.0 \pm 20.1 \\
25.1 \pm 3.5\end{array}$ & $\begin{array}{l}343.4 \pm 17.3 \\
5.8 \pm 0.6\end{array}$ & $\begin{array}{l}410.9 \pm 53.2 \\
4.6 \pm 0.5 \\
\end{array}$ & $\begin{array}{l}573.0 \pm 56.5 \\
65.7 \pm 8.6\end{array}$ & $\begin{array}{l}427.0 \pm 37.3 \\
37.5 \pm 7.9\end{array}$ & $\begin{array}{l}0.001 \\
<0.001\end{array}$ \\
\hline Crotonaldehyde & HPMMA & $606.3 \pm 70.5$ & $240.3 \pm 32.3$ & $256.6 \pm 50.5$ & $1,347.0 \pm 185.2$ & $838.7 \pm 168.2$ & $<0.001$ \\
\hline $\begin{array}{l}\mathrm{N}, N- \\
\text { Dimethylformamide }\end{array}$ & AMCC & $284.8 \pm 22.3$ & $154.2 \pm 13.2$ & $227.5 \pm 34.0$ & $526.1 \pm 52.9$ & $336.8=$ & $<0.001$ \\
\hline $\begin{array}{l}\text { Ethylbenzene, } \\
\text { styrene }\end{array}$ & PGA & $290.6 \pm 15.5$ & $234.0 \pm 17.2$ & $220.2 \pm 27.7$ & $407.9 \pm 36.5$ & $337.2 \pm 37.0$ & $<0.001$ \\
\hline Propylene Oxide & 2HPMA & $61.8 \pm 8.8$ & $50.3 \pm 17.8$ & $57.4 \pm 21.8$ & $87.9 \pm 10.0$ & $60.3 \pm 10.4$ & $<0.001$ \\
\hline Styrene & MA & $261.0 \pm 16.5$ & $171.9 \pm 14.9$ & $180.0 \pm 21.0$ & $391.5 \pm 40.2$ & $371.6 \pm 33.8$ & $<0.001$ \\
\hline Toluene & BMA & $15.1 \pm 2.1$ & $15.2 \pm 3.2$ & $11.7 \pm 3.4$ & $18.1 \pm 6.1$ & $14.5 \pm 3.7$ & 0.752 \\
\hline Xylene & $\begin{array}{l}2 \mathrm{MHA} \\
3 \mathrm{MHA}+ \\
4 \mathrm{MHA}\end{array}$ & $\begin{array}{l}56.4 \pm 7.7 \\
403.9 \pm 46.1\end{array}$ & $\begin{array}{l}22.2 \pm 4.5 \\
250.1 \pm 76.3\end{array}$ & $\begin{array}{l}41.8 \pm 13.4 \\
275.9 \pm 85.3\end{array}$ & $\begin{array}{l}131.6 \pm 24.5 \\
725.5 \pm 90.2\end{array}$ & $\begin{array}{l}56.0 \pm 12.0 \\
473.5 \pm 72.3\end{array}$ & $\begin{array}{l}<0.001 \\
<0.001\end{array}$ \\
\hline Anabasine & ANB & $4.7 \pm 0.4$ & $2.8 \pm 0.3$ & $4.5 \pm 1.1$ & $7.5 \pm 1.0$ & $5.6 \pm 0.9$ & $<0.001$ \\
\hline Anatabine & ANTB & $3.1 \pm 0.5$ & $0.8 \pm 0.1$ & $1.1 \pm 0.3$ & $8.1 \pm 1.5$ & $4.0 \pm 1.4$ & $<0.001$ \\
\hline Nicotine & $\begin{array}{l}\mathrm{NIC} \\
\mathrm{COT} \\
3 \mathrm{HC}\end{array}$ & $\begin{array}{l}657.2 \pm 130.4 \\
527.0 \pm 91.6 \\
1,716.0 \pm 258.2\end{array}$ & $\begin{array}{l}11.3 \pm 2.4 \\
2.3 \pm 0.3 \\
9.5 \pm 3.1\end{array}$ & $\begin{array}{l}13.9 \pm 2.9 \\
2.6 \pm 0.6 \\
11.7 \pm 3.7 \\
\end{array}$ & $\begin{array}{l}1,350.4 \pm 287.6 \\
1,413.8 \pm 242.4 \\
4,234.2 \pm 453.8\end{array}$ & $\begin{array}{l}1,795.5 \pm 443.8 \\
1,081.3 \pm 215.7 \\
3,937.7 \pm 682.6\end{array}$ & $\begin{array}{l}<0.001 \\
<0.001 \\
<0.001\end{array}$ \\
\hline
\end{tabular}

Values = mean \pm SE. Abbr.: AAMA, N-acetyl-S-(2-carbamoylethyl)-L-cysteine; AMCC, N-acetyl-S-(N-methylcarbamoyl)-L- 
cysteine; ANB, anabasine; ANTB, anatabine; BMA, N-acetyl-S-(benzyl)-L-cysteine; CEMA, N-acetyl-S-(2-carboxyethyl)-L-cysteine; CIG, cigarette; COT, cotinine; DHBMA, N-acetyl-S-(3,4-dihydroxybutyl)-L-cysteine; E-cig, electronic cigarette; 3HC, trans-3'hydroxycotinine; 2HPMA, N-acetyl-S-(2-hydroxypropyl)-L-cysteine; 3HPMA, N-acetyl-S-(3-hydroxypropyl)-L-cysteine; HPMMA, N-acetyl-S-(3-hydroxypropyl-1-methyl)-L-cysteine; MA, mandelic acid; 2MHA, 2-methylhippuric acid; 3MHA, 3-methylhippuric acid; 4MHA, 4-methylhippuric acid; 3MHBMA, N-acetyl-S-(4-hydroxy-2-buten-1-yl)-L-cysteine; NIC, nicotine; PGA, phenylglyoxylic acid. 
metabolites of tobacco alkaloids (nicotine (NIC), cotinine (COT), and trans-3'hydroxycotinine (3HC)) were significantly increased in cigarette only users and e-cig or dual users compared with never or former smokers, but levels were not significantly different between the two current product use categories (Table 3.5).

\section{Tobacco Product Use Categories and CACs}

In the fully adjusted (adjusted for age, sex, CVD history, use of CVD medications, family history of CVD, SHS exposure, and SES (income category used as proxy indicator)) multivariable-linear regression model testing the association between tobacco product use categories and log-transformed CAC levels, levels of CAC-12 (CD45+) were significantly decreased in e-cig or dual users compared with never smokers $(-6.6 \% ; p=0.008)$ (Table 3.6). There were no significant differences in the levels of the other fourteen measured CAC populations between any of the tobacco product use categories (Table 3.6). In order to explore dimension reduction of the demographic adjustments used in the fully adjusted model, a random forest model was used to select the demographic variables that varied the most across the four tobacco product use categories independently of smoking status. Based on the resulting variable importance plot (Fig. 3.3), multivariable-adjusted linear regression models were again used to assess the relationship between log-transformed CAC measures and tobacco product use categories adjusting for age and SES. When the model was adjusted only for age and SES, levels of CAC-12 (CD45+) remained decreased in ecig or dual users compared with never smokers $(-6.4 \% ; \mathrm{p}=0.004)$, and CAC-3 (CD31+/CD34+/AC133+/CD45dim) was also found to be significantly different between never smokers and e-cig or dual users, with e-cig or dual users showing significantly increased levels $(+120 \%$; $\mathrm{p}=0.03)$ (Table 3.7, Fig. 3.3). 
Table 3.6. Percent change in levels of circulating angiogenic cells (CACs) comparing tobacco product use categories with never smokers in the fully adjusted model.

\begin{tabular}{|c|c|c|c|}
\hline CAC & $\begin{array}{c}\text { Former Smoker, } \\
\%(95 \% \mathrm{CI})\end{array}$ & $\begin{array}{l}\text { CIG Only, } \\
\%(95 \% \text { CI })\end{array}$ & $\begin{array}{c}\text { E-cig or Dual User, } \\
\%(95 \% \mathrm{CI})\end{array}$ \\
\hline CAC-1 (CD31+/CD34+/CD45dim) & $15.5(-20.2$ to 67.0$)$ & $-5.8(-33.2$ to 32.8$)$ & $4.3(-30.7$ to 56.9$)$ \\
\hline $\mathrm{CAC}-2\left(\mathrm{CD}_{4}+/ \mathrm{CD34}_{+} / \mathrm{CD31}+\right)$ & $2.3(-29.9$ to 49.4$)$ & $-1.3(-30.6$ to 40.4$)$ & $-15.4(-44.3$ to 28.6$)$ \\
\hline CAC-3 (CD31+/CD34+/AC133+/CD45dim) & $44.0(-29.0$ to 192.1$)$ & $8.0(-44.1$ to 108.6$)$ & $75.8(-19.6$ to 284.4$)$ \\
\hline CAC-4 (AC133+/CD34+/CD31+/CD45+) & $-15.9(-50.0$ to 41.2$)$ & $-14.9(-47.5$ to 37.9$)$ & $-7.0(-47.6$ to 65.0$)$ \\
\hline CAC-5 (AC133+/CD31+) & $8.0(-32.6$ to 73.1$)$ & $-17.3(-46.7$ to 28.2$)$ & $-25.9(-56.0$ to 24.8$)$ \\
\hline CAC-6 (CD31+/CD34+) & $-3.9(-40.0$ to 54.0$)$ & $-20.0(-48.4$ to 24.1$)$ & $-30.2(-58.6$ to 17.6$)$ \\
\hline CAC-7 (CD31+/CD34+/AC133-/CD45dim) & $13.9(-21.2$ to 64.7$)$ & $-5.9(-33.2$ to 32.6$)$ & $1.9(-32.2$ to 53.2$)$ \\
\hline CAC-8 (CD45+/CD34+/CD31+/AC133-) & $5.1(-27.9$ to 53.2$)$ & $0.9(-29.0$ to 43.2$)$ & $-16.1(-44.7$ to 27.3$)$ \\
\hline CAC-9 (CD34+) & $-6.3(-32.8$ to 30.6$)$ & $-8.8(-33.0$ to 24.2$)$ & $-7.9(-36.2$ to 32.9$)$ \\
\hline CAC-10 (CD31+) & $17.8(-10.5$ to 55.1$)$ & $8.3(-16.1$ to 39.8$)$ & $-8.7(-32.6$ to 23.7$)$ \\
\hline CAC-11 (AC133+) & $25.3(-13.7$ to 81.9$)$ & $-1.9(-30.6$ to 38.8$)$ & $-24.1(-49.8$ to 14.7$)$ \\
\hline CAC-12 (CD45+) & $1.3(-3.1$ to 5.9$)$ & $-2.8(-6.7$ to 1.3$)$ & $-6.6(-11.1$ to -1.9$)$ \\
\hline $\mathrm{CAC}-13\left(\mathrm{CD34}_{+} / \mathrm{ACC133}_{+}\right)$ & $-6.0(-38.5$ to 43.7$)$ & $-0.3(-32.8$ to 48.0$)$ & $18.8(-25.7$ to 89.9$)$ \\
\hline $\mathrm{CAC}-14\left(\mathrm{AC}^{233+/ C D 34+/ C D 45+)}\right.$ & $-10.0(-40.3$ to 35.7$)$ & $-12.5(-40.2$ to 28.2$)$ & $14.7(-27.2$ to 80.6$)$ \\
\hline CAC-15 (CD34+/ACC133+/CD45dim) & $10.6(-28.9$ to 72.1$)$ & $-3.0(-35.7$ to 46.4$)$ & $26.5(-22.5$ to 106.4$)$ \\
\hline
\end{tabular}


Estimates were derived using the formula $(\mathrm{e} \beta-1) \times 100 \%$, where $\beta$ represents the $\beta$-coefficient of the multivariable-adjusted association between product use categories and ln (CAC levels). Models adjusted for age, sex, cardiovascular disease history, use of cardiovascular medications, family history of CVD, secondhand smoke exposure, and socioeconomic status (income category used as proxy indicator). Bolded values are statistically significant at p<0.05. Abbr.: CAC, circulating angiogenic cell; CI, confidence interval; CIG, cigarette; E-cig, electronic cigarette. 


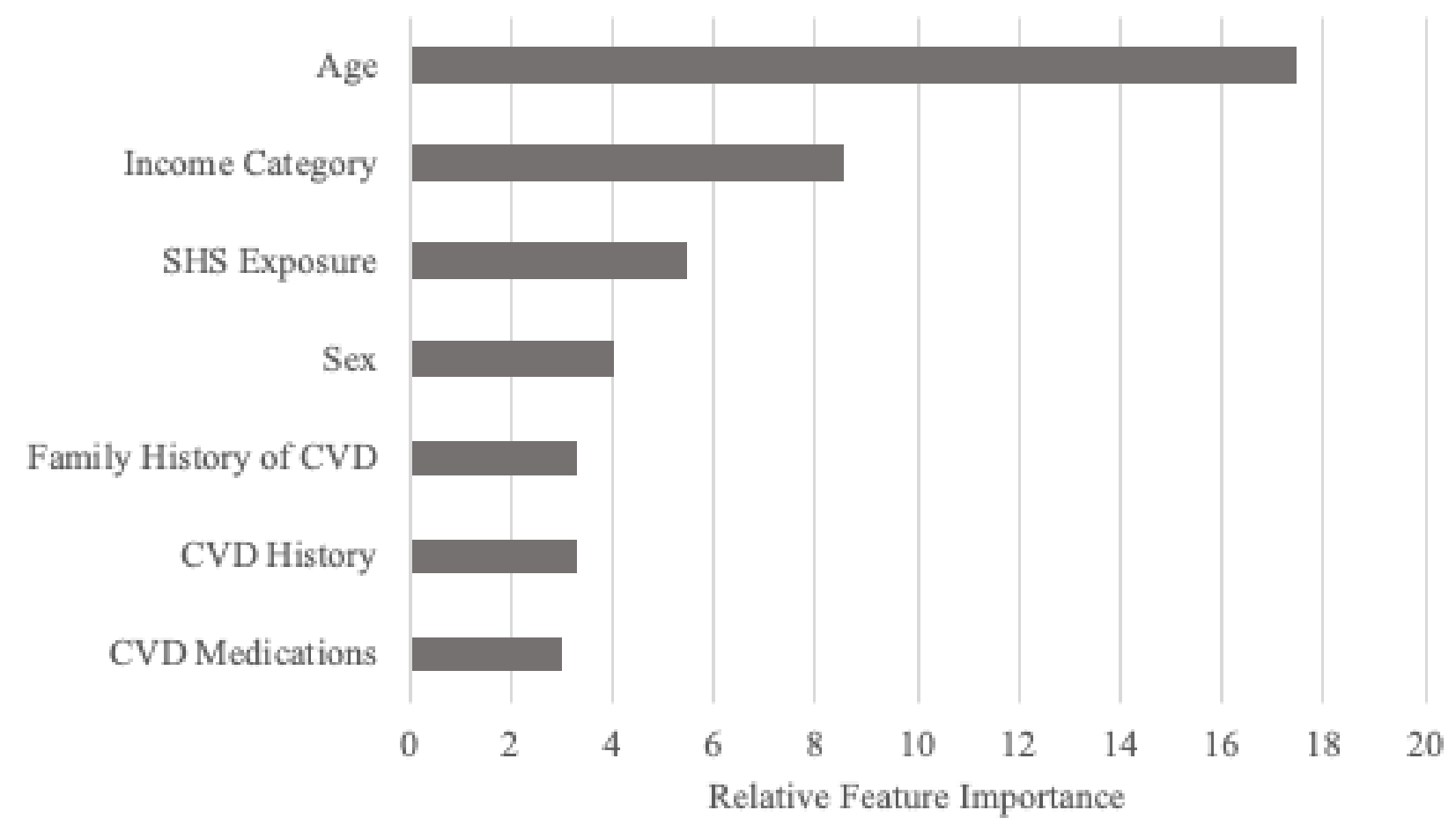

Fig. 3.2. Relative feature importance of demographic variables on levels of circulating angiogenic cells (CACs). A random forest model was used to determine the most important demographic variables influencing levels of CACs independently of tobacco product use category. Importance was calculated by reduction of gini impurity at relevant branchings. Abbr.: CAC, circulating angiogenic cell; CVD, cardiovascular disease; SHS, secondhand smoke. 
Table 3.7. Percent change in levels of circulating angiogenic cells (CACs) comparing tobacco product use categories to never smokers in the secondary adjusted model.

\begin{tabular}{|c|c|c|c|}
\hline $\mathbf{C A C}$ & $\begin{array}{c}\text { Former Smoker, } \\
\%(95 \% \mathrm{CI})\end{array}$ & $\begin{array}{l}\text { CIG Only, } \\
\%(95 \% \text { CI })\end{array}$ & $\begin{array}{c}\text { E-cig or Dual User, } \\
\%(95 \% \mathrm{CI})\end{array}$ \\
\hline CAC-1 (CD31+/CD34+/CD45dim) & $23.7(-13.3$ to 76.4$)$ & $-4.6(-31.4$ to 32.8$)$ & $10.8(-22.6$ to 58.7$)$ \\
\hline CAC-2 (CD45+/CD34+/CD31+) & $4.7(-26.8$ to 49.9$)$ & $-1.4(-29.4$ to 37.7$)$ & $-13.3(-39.7$ to 24.6$)$ \\
\hline CAC-3 (CD31+/CD34+/AC133+/CD45dim) & $38.5(-29.8$ to 173.2$)$ & $24.5(-33.8$ to 134.2$)$ & $120.3(10.8$ to 337.8$)$ \\
\hline CAC-4 (AC133+/CD34+/CD31+/CD45+) & $-20.2(-51.2$ to 30.6$)$ & $-8.3(-42.0$ to 44.9$)$ & $11.3(-32.4$ to 83.1$)$ \\
\hline CAC-5 (AC133+/CD31+) & $7.2(-31.5$ to 67.8$)$ & $-15.8(-44.5$ to 27.7$)$ & $-19.1(-48.6$ to 27.2$)$ \\
\hline CAC-6 (CD31+/CD34+) & $0.6(-35.6$ to 57.4$)$ & $-18.9(-46.5$ to 23.0$)$ & $-28.0(-54.2$ to 13.2$)$ \\
\hline CAC-7 (CD31+/CD34+/AC133-/CD45dim) & $23.1(-13.7$ to 75.7$)$ & $-5.7(-32.3$ to 31.2$)$ & $6.9(-25.4$ to 53.1$)$ \\
\hline CAC-8 (CD45+/CD34+/CD31+/AC133-) & $8.6(-24.1$ to 55.3$)$ & $0.0(-28.3$ to 39.5$)$ & $-15.1(-40.9$ to 21.9$)$ \\
\hline CAC-9 (CD34+) & $-3.9(-29.9$ to 31.7$)$ & $-8.9(-32.0$ to 22.2$)$ & $-8.8(-33.7$ to 25.5$)$ \\
\hline $\mathrm{CAC}-10(\mathrm{CD31}+)$ & $19.2(-8.4$ to 55.1$)$ & $9.1(-14.6$ to 39.4$)$ & $-2.2(-25.1$ to 27.6$)$ \\
\hline CAC-11 (AC133+) & $21.5(-15.0$ to 73.6$)$ & $0.7(-27.7$ to 40.4$)$ & $-17.4(-42.4$ to 18.5$)$ \\
\hline CAC-12 (CD45+) & $0.5(-3.7$ to 4.9$)$ & $-2.4(-6.2$ to 1.5$)$ & $-6.4(-10.3$ to -2.2$)$ \\
\hline CAC-13 (CD34+/ACC133+) & $-7.0(-38.1$ to 39.5$)$ & $5.4(-27.8$ to 53.7$)$ & $35.5(-10.1$ to 104.3$)$ \\
\hline CAC-14 (AC133+/CD34+/CD45+) & $-13.6(-41.5$ to 27.4$)$ & $-9.8(-37.2$ to 29.5$)$ & $25.4(-15.4$ to 85.8$)$ \\
\hline CAC-15 (CD34+/ACC133+/CD45dim) & $10.0(-28.2$ to 68.5$)$ & $3.6(-30.3$ to 54.1$)$ & $44.8(-5.9$ to 122.9$)$ \\
\hline
\end{tabular}


Estimates were derived using the formula $(\mathrm{e} \beta-1) \times 100 \%$, where $\beta$ represents the $\beta$-coefficient of the multivariable-adjusted association between product use categories and $\ln$ (CAC levels). Models adjusted for age and socioeconomic status (income category used as proxy indicator). Bolded values are statistically significant at $\mathrm{p}<0.05$. Abbr.: CAC, circulating angiogenic cell; CI, confidence interval; CIG, cigarette; E-cig, electronic cigarette. 


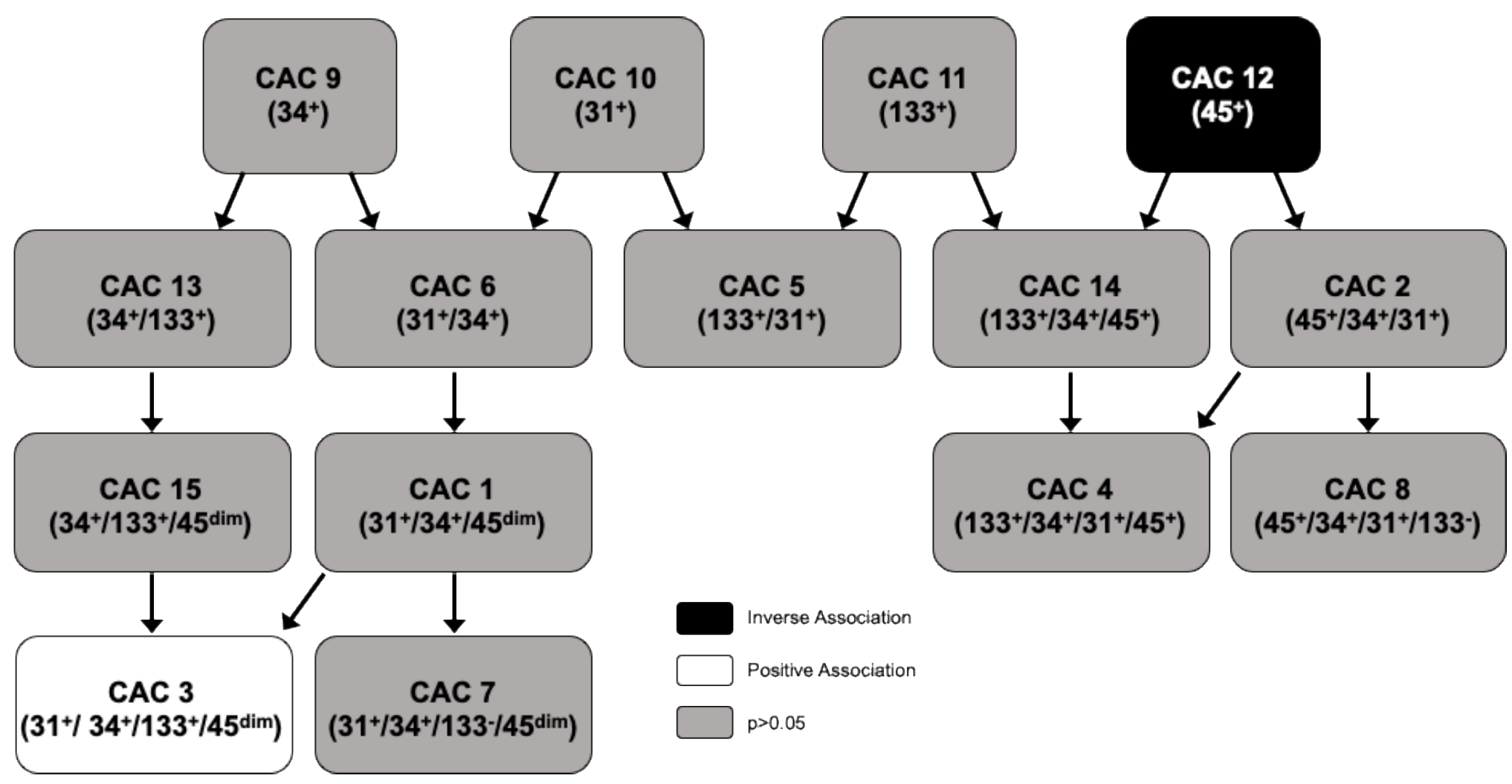

Fig. 3.3. Interrelationships between circulating angiogenic cells (CACs) associated with e-cig or dual use compared with never smokers. Levels of 15 antigenetically defined populations of CACs were measured in 103 participants and examined for their association with tobacco product use categories adjusted for age and socioeconomic status. Associations were only found between ecig or dual users and CAC-3 (31+/34+/133+/45dim) and CAC-12 (45+) compared with levels of CACs measured in never smokers. 


\section{Discussion}

The Louisville Healthy Heart Study represents one of the first human cohort studies to examine the associations between a large number of CAC populations, urinary VOC and tobacco alkaloid metabolites, and specific types of tobacco product use, including e-cig or dual e-cig and traditional cigarette users. Although many adults are using e-cigs as an alternative to traditional cigarettes (325), there has been a marked increase in e-cig use among youths $(83,182,183)$. Although the current study cohort did not include any individuals under the age of 18 (based on exclusion criteria), this trend in the use of e-cig devices is reflected in our cohort, as e-cig or dual users were significantly younger than never and former smokers and cigarette only users. The use of e-cigs by younger individuals - and especially individuals who may not have previously been using tobacco products - is a cause for concern as e-cigs have the potential to create a new generation of individuals addicted to nicotine (326). The current cohort also contained a greater number of females than males (66\% vs $34 \%$ ), although this ratio was not statistically significant. As males are more likely than women to be smokers (83), there may exist relationships between tobacco product users and the measured outcomes that were not noted due to the imbalance of sex in the participants in the current cohort. Use of tobacco products has also previously been associated with lower education levels and lower household incomes, as was seen in our cohort (83).

Likely due to their younger age, e-cig or dual users were found to have a lower prevalence of high blood cholesterol and hypertension than never smokers, former smokers, or cigarette only users and to have a lower usage of CVD medicines. Increased blood pressure and levels of cholesterol are known to increase with age $(327,328)$, so it is 
unsurprising that the youngest participants on average in this study have less prevalence of major cardiovascular conditions. Perhaps also unsurprising is the significantly lower prevalence of hypertension in never smokers than in former smokers as well as the lower use of beta-blockers, which are generally prescribed to help lower blood pressure. Although several epidemiological studies have paradoxically shown decreased blood pressure in smokers compared with nonsmokers or former smokers (329-332), smoking is still considered a risk factor for hypertension (333-335). However, there is evidence associating previous smoking with increased blood pressure, as former smokers have been shown to have increased blood pressure and hypertension compared with never smokers $(331,336)$. This may be due to the development of arterial stiffness, which is closely linked with the development of hypertension (337) and which has been shown to be exacerbated by smoking (338-341). Although this increased arterial stiffness has been shown to be reversible, it may take more than a decade to achieve levels of stiffness similar to those found in never smokers (342). This persistent stiffness even after smoking cessation may be an important factor in the lower prevalence of hypertension seen in our never-smoking participants compared with those who had formerly smoked.

Hematological measures showed significantly increased leukocytes in e-cig or dual users compared with never smokers, driven by an increase in granulocytes, although the specific type(s) of granulocyte being affected—neutrophils, eosinophils, or basophils—is unknown. It is likely, however, that this increase is at least in part due to increased neutrophils, an increase that has been associated with the use of cigarettes (343-347), as neutrophils serve as mediators of inflammation (348-350). Although leukocytes were increased in cigarette only users, this increase was not significant compared with never 
smokers. Several epidemiological studies have found increased levels of leukocytes in cigarette smokers $(345,351-355)$, a change likely indicative of the persistent inflammation induced by MCS $(345,354,356-358)$, and while the effects of e-cigs on hematological measures are still not well defined, a small study of cigarette smokers and never-smokers compared $\mathrm{CBC}$ markers after cigarette and e-cig use and found that although leukocytes, lymphocytes, and granulocytes were increased after cigarette smoking, they were unchanged after e-cig use (359). It is therefore likely that the increase in leukocytes seen in our e-cig or dual use group is mostly contributable to increased levels of leukocytes in individuals using both traditional and emerging tobacco products.

Measures by $\mathrm{CBC}$ also showed increased hemoglobin in e-cig or dual users. Although some prior studies have shown increases in hemoglobin in smokers $(339,349$, 354), these changes were not seen in the current study. As previously mentioned, there are few studies of the hematological impacts of e-cig use, but Okuni-Watanabe et al. (360) report a case study showing that hemoglobin, as well as hematocrit, was initially increased in an e-cig user but decreased upon the cessation of e-cig use. Although this case report was based on a single individual—an individual who was also a former heavy smoker-it does suggest the potential for e-cig-related changes in erythrocytic measures. Additional studies are needed to better clarify the effects of e-cig use on hematological measures in both e-cig only users and in dual users.

Red blood cell distribution width (RDW) was found to be significantly higher in cigarette only users compared with both never smokers and e-cig or dual users. Elevated RDW has been previously seen in smokers (361-363) and has been associated with increased morbidity and mortality $(362,364-367)$. Although the mechanism between 
RDW and subsequent morbidity and mortality has not been clarified, this finding in our study may be predictive of future cardiovascular events in the cigarette-smoking participants. Contrastingly, platelet mononuclear cell aggregates (PMAs), a marker of increased thrombosis, were found to be significantly decreased in e-cig or dual users compared with all other groups. Smoking has been long been known to increase platelet aggregation (368), as well as platelet leukocyte aggregation (369), and studies of e-cig exposure have shown similar outcomes (370-372). However, although our e-cig or dual user participants showed no significant difference in platelet count (as measured by $\mathrm{CBC}$ ) compared with any other use group, they showed decreased PMAs. The reason for this decrease is unknown, and future studies of cigarette only, e-cig only, and dual users should continue to measure PMAs and measure additional markers of thrombosis to better understand this finding.

Unsurprisingly, most urinary VOC metabolites showed higher levels in current tobacco product users compared with never and/or former smokers. Cigarettes are a known source of VOCs (160) as well as one of the leading sources of nicotine (373), and both cigarette only users and e-cig or dual users also showed increased levels of tobacco alkaloids compared with both never and former smokers. Concentrations of nicotine can vary in e-cigs, as different flavorings can contain different levels of nicotine (374), as can levels of VOCs in relation to varying power outputs $(96,375)$, making measurements of these compounds in e-cig aerosols difficult. However, several studies have shown that, as in the current study, levels of VOCs generated by e-cig devices are lower than levels generated by MCS but still increased compared with levels in nonsmokers (180, 207, 211, 375). Our study supports these findings, as several urinary VOC metabolites were 
significantly lower in e-cig or dual users compared with cigarette only smokers, including both metabolites of acrolein (CEMA and 3HPMA) and the metabolite of crotonaldehyde (HPMMA), despite similar levels of urinary cotinine. Although these data support the hypothesis that e-cigs may be less harmful than traditional cigarettes, they also contribute to the growing body of evidence against the claims that e-cigs are harmless $(227,376-378)$. More studies are needed to better clarify levels of VOCs and tobacco alkaloids generated by e-cigs compared with traditional cigarettes and to determine whether or not any differences in levels between the two types of products indicate a decreased risk of tobacco product-related CVD.

CACs were also examined in relation to tobacco product use categories. Previous research has shown associations between MCS and CACs $(72,316,318,320)$ as well as between acrolein, one of the major constituents of tobacco smoke, and CACs $(71,74,151)$. In our models, age and SES were identified as variables affecting levels of CACs independent of tobacco product use categories. Age is a known risk factor for CVD and is included as a variable in a number of CVD risk score calculations (379-382). Age is also associated with decreased endothelium function (383-385), likely through decreased levels and function of CACs $(385,386)$. Likewise, SES has been shown to have a significant impact on CVD health (387), with this impact thought to be the result of factors such as poor diet (388), decreased access to health care (389), increased presence of roadways (294), and decreased levels of greenness (295) or some combination of these factors and/or others. Both greenness (295) and presence of roadways (294) have previously been associated with changes in the levels of CACs.

Of the fifteen CAC populations measured in our cohort, only two showed 
significant associations with any of the tobacco product use categories compared with never smokers after adjustment for age and SES: CAC-12 (45+) and CAC-3 $(31+/ 34+/ 133+/ 45 \mathrm{dim})$. CAC-12 is a circulating hematopoietic cell which expresses the leukocyte antigen CD45. In our cohort, e-cig or dual users had significantly lower levels of these cells than never smokers. Although exposure to MCS is associated with decreased levels of CACs, there are still gaps in our understanding of the effect of e-cigs on CACs, particularly populations such as CAC-12, which is a hematopoietic cell but not a hematopoietic stem cell (CD45+/CD34+/CD31+) or an endothelial progenitor cell (CD45 $\left.\mathrm{dim} / \mathrm{CD} 34+/ \mathrm{CD} 31_{+}\right)$. In a previous study, Yeager et al. (295) found that CAC-12, among many of the measured CAC populations, was inversely associated with greenness while other CAC populations derived from CAC-12 (CAC-14 and CAC-4) were positively associated. The presence of the CD45 antigen appeared to contribute to the reparative capacity of the cells positively associated with nearby greenness, as cells displaying the same endothelial and stem cell markers (CD31+/CD34+/133+) but not displaying the CD45 antigen (CD45- or CD45dim) did not show the same response (295). Additional research is needed in order to better understand the true significance of the CD45 antigen in CACs and its potential contribution to the regenerative and reparative capacities of hematopoietic stem cells.

Conversely, the results of our study showed an increase in CAC-3 in e-cig or dual use compared with no product use, past or present. Although smoking has been found to decrease level of CACs, it may be that, as seen with higher levels of roadway proximity (294), the increased levels of CAC-3 measured in e-cig or dual users are indicative of a low yet persistent level of vascular injury that necessitates increased levels of this early 
progenitor cell population for repair of the endothelium. This insult may be mild enough to induce increased production of CAC-3 without affecting cell mobilization from the bone marrow, an outcome noted in response to exposure to $\mathrm{PM}_{2.5}$ (67) and acrolein (74). Regardless, as levels of the $\mathrm{CAC}$ populations that give rise to $\mathrm{CAC}-3$ were not significantly affected by the use of cigarettes and/or e-cigs, the changes noted in CAC-3 may indicate that this population is particularly sensitive to some component to which e-cig or dual users are exposed.

Interestingly, a study of CACs in type 2 diabetics showed that of the CAC populations measured, only CAC-3 was associated with peripheral endothelium function as measured by reactive hyperemia index (RHI), with individuals with higher levels of CAC-3 showing better endothelium function (390). CAC-3 was thus considered the CAC most reflective of endothelium health and was suggested as a specifically sensitive predictor of CVD risk (390). These data also seem to support the hypothesis that the increased levels of CAC-3 in e-cig or dual users compared with never smokers is reflective of increased endothelium function as the vasculature works to repair low levels of injury. Similar increases in CACs are noted in response to acute exposure to e-cig aerosol (322) as well as to MCS (315) and PM $(73,391)$, while chronic exposures to MCS or PM cause decreases in CACs as endothelium damage overwhelms repair capabilities. As e-cig use is still a fairly acute phenomenon — that is, the majority of users have not been using e-cigs long enough to develop any potential adverse health outcomes that may be associated with chronic use - the increased levels of CAC-3 noted in our study may be reflective of the increase in CACs related to acute exposure, while the increase in dual users may be reflective of the increases in CACs noted with lighter cigarette use (318). Additional 
studies of more chronic e-cig and dual use would help clarify whether this increase persists or whether, as with MCS and PM, the levels of CAC-3 decrease.

Given that both these cell populations were only associated with the e-cig or dual use group, we cannot definitively say whether these changes were induced by sole e-cig use or by the combined use of cigarettes and e-cigs. However, as no significant differences were seen in the level of these CACs between cigarette only smokers and never smokers, it is likely that the changes seen in CAC-3 and CAC-12 are related more so to the use of ecigs. Additional studies with a larger population of e-cig users are needed to better understand these relationships, particularly the relationship with CAC-3, which may specifically have the potential to serve as a biomarker for endothelial function and disease development as previously suggested (390).

The Louisville Healthy Heart Study represents a cross-sectional study examining associations between the use of tobacco products and fifteen antigenetically distinct CAC populations. Although our small number of e-cig only users required their inclusion in an 'e-cig or dual use' group and thus somewhat limited our capability of thoroughly assessing the effect of these devices on CACs as well as on urinary VOC and tobacco alkaloid metabolites and hematological measures, a number of significant differences were noted between this group and the group of individuals smoking cigarettes alone. These differences suggest that e-cig use may produce harmful effects in these outcomes that are distinct from those seen in response to use of cigarettes in addition to the outcomes showing similar responses, such as increased leukocytes. Furthermore, as most e-cig users are actually dual users $(392,393)$, the results of our combined e-cig or dual use group may be more reflective of real-world exposure to e-cigs and subsequent health outcomes. Our e- 
cig or dual user group was significantly younger than other use groups, supporting national studies reporting increased use of e-cigs among younger generations $(182,183,394)$, and reported less prevalence of CVD, likely related to their age. Because a number of participants were recruited from CVD clinics at nearby hospitals, the prevalence of CVD and use of CVD medications were unsurprisingly similar between never smokers, former smokers, and cigarette smokers.

Also unsurprising were the significantly higher levels of urinary VOC metabolites in both groups of current tobacco product use compared with never and former smokers, as tobacco products are known to be a significant source of VOCs (160). However, comparison of urinary VOC metabolite levels between cigarette only smokers and e-cig or dual users showed that the use of cigarettes alone resulted in significantly higher levels of several metabolites (CEMA, 3HPMA, AAMA, 3MHBMA, HPMMA, AMCC, and ANTB). Nonetheless, the levels of these metabolites were still significantly higher in ecig or dual users compared with never or former smokers, so a decreased but continued use of traditional cigarettes in combination with the use of e-cigs will likely still result in levels of VOC exposure that are capable of contributing to CVD development. Likewise, because e-cigs are often considered to be safer than traditional cigarettes $(173,175,181)$, e-cig only users may not be aware of the high levels of VOCs to which they are being exposed and the associated health risks.

Analysis of CACs revealed that two cell populations were significantly different between e-cig or dual users and never smokers. CAC-3, an early endothelial progenitor cell population, was significantly increased in e-cig or dual users, while CAC-12, a hematopoietic cell, was significantly decreased. The increased levels of CAC-3 are likely 
reflective of a repair mechanism in response to a low but persistent level of injury in the endothelium. The cause in the decrease in levels of CAC-12, despite being strongly associated with e-cig or dual use as evidenced by its significance in all tested models, is less clear and will require additional, more specific studies to better understand. That both these differences were significant despite a small sample in our e-cig or dual use group speaks to their importance and potentially to their relevance in other CVD effects induced by the use of e-cigs alone or in combination with traditional cigarettes.

With the increasing use of e-cigs and other ENDS, it is essential to understand the health risks associated with their use. Although few studies have examined the risks of ecigs on cardiovascular health, the changes noted in hematological measures and in CACs in the LHHS and in other human cohorts (322) demonstrate the potential for these devices to contribute to increased CVD risk, as does the evidence of high levels of VOCs in e-cig aerosol both in our study and in others (395). Additional research is needed to clarify the results of these early e-cig studies and to explore the specific findings shown in the current study. Furthermore, more studies focusing on e-cig and dual users would help elucidate how the combined use of e-cigs and traditional cigarettes may change CVD risk, that is, whether combined use is healthier or safer than use of cigarettes alone. A better understanding of the impacts of e-cigs on overall health would help medical professionals develop better methods of care for both acute and chronic adverse effects resulting from the use of these products. 


\section{CHAPTER IV}

\section{SYSTEMIC, HEMATOLOGICAL, AND ENDOTHELIUM-RELATED EFFECTS OF MAINSTREAM CIGARETTE SMOKE, E-CIGARETTE AEROSOL, AND PROPYLENE GLYCOL:VEGETABLE GLYCERIN IN MICE}

\section{A. Introduction}

Cardiovascular disease (CVD) is the leading cause of morbidity and mortality worldwide $(1,2)$. In 2016, more than one-fourth of the almost 57 million global deaths were attributed to CVD, and ischemic heart disease and stroke have been classified as the two leading causes of death for the last 20 years (2). In the United States alone, on average, an individual dies of CVD every 38 seconds; this means that more than 2,000 individuals die every day from some form of CVD (1). Although a number of physical, behavioral, and socioeconomic factors have been linked to CVD (3), tobacco smoke has been characterized as the most significant modifiable risk factors in CVD development $(14,75)$. Tobacco smoke contains thousands of compounds, including up to 100,000 unidentified compounds (396), and many of these can contribute to disease development even in very small quantities $(82,396)$. Extensive evidence demonstrates that exposure to both mainstream (MCS) $(80,82)$ and secondhand (SHS) $(80,84)$ cigarette smoke increase the risk for CVD. Smoking has been shown to increase the risk of heart attack, coronary artery disease, atherosclerosis, and stroke (85). Smoking has also been linked to endothelium 
dysfunction (85), a sine qua non for CVD. However, the pathophysiological mechanisms through which tobacco smoke mediates and promotes CVD are not well understood.

Although American smoking rates are decreasing $(1,119)$, the number of individuals using electronic cigarettes (e-cigs) is increasing, especially among youths and never smokers $(1,182,183,325,397)$, and although e-cigs are promoted as being healthier than traditional cigarettes $(173-175,177,184)$, their true health effects are not yet known. Early studies of e-cigs have shown that the generated aerosol can contain many harmful or potentially harmful components, including particulates $(184,198-202)$, metals $(184,203$ 205), and volatile organic compounds (VOCs) including aldehydes (96, 180, 199, 201, 206-213). The potential health effects of the liquid components are also causing growing concern, as humectants including propylene glycol (PG) and vegetable glycerin (VG) have been linked to aldehyde generation $(168,214-216)$ and as many of the flavoring additives used to make flavored e-cig liquids have been deemed safe for ingestion but not necessarily for inhalation (218). As the morbidity and mortality from e-cigarette, or vaping, product use associated lung injury (EVALI) has increased in the United States over the past year (225-229), it has become imperative to study the health effects of e-cigs and their various components. Thus, the goal of these experiments was to determine the effects of MCS, ecig aerosol, and PG:VG on CACs as well as systemic and hematological biomarkers.

\section{B. Experimental Procedures}

\section{Exposures}

Animals were exposed to mixtures or humectants via full-body inhalation as described in Chapter II, Sections B.2. Upon completion of the exposure, mice were euthanized and tissues collected as described in Chapter II, Section B.5. 


\section{Urine Collection}

Urine samples were collected post-exposure as described in Chapter II, Section B.3.

\section{Urine Metabolite Quantification}

Collected urine was used to quantify levels of VOC and tobacco alkaloid metabolites using analytical methods as described in Chapter II, Section B.4.

\section{Complete Blood Cell Count (CBC)}

A sample of whole blood was collected after the final day of exposure for a complete blood cell count (CBC) as described in Chapter II, Section B.6.

\section{Plasma Biomarkers Quantification}

Plasma was prepared from each sample for an analysis of a number of biomarkers as described in Chapter II, Section B.7.

\section{Circulating Angiogenic Cell (CAC) Quantification}

Flow cytometry was used to analyze the number of circulating angiogenic cells (CACs) in each sample as described in Chapter II, Section B.8.

\section{Statistical Analyses}

Statistical analyses were performed as described in Chapter II, Section B.10.

\section{Results}

\section{Urinary Metabolites in MCS-Exposed Mice}

The use of tobacco products is the leading cause of preventable death both nationally and worldwide (1). Mainstream cigarette smoke (MCS) has been shown to contain thousands of compounds (396), and many of these have been implicated in disease development $(80-82,396)$. To better understand how specific components of MCSmainly, aldehydes - may be contributing to disease development, specifically CVD, we 
measured urinary formate, acetate, 3-hydroxypropylmercapturic acid (3HPMA), and 3hydroxy-1-methylpropylmercapturic acid (HPMMA), as well as total urinary nicotine metabolites (nicotine, cotinine, and trans-3'-hydroxycotinine (3HC)) by gas chromatography/mass spectrometry (GC-MS) or ultra-performance liquidchromatography tandem mass spectrometry (UPLC-MS/MS) as biomarkers of MCSrelated aldehyde exposure. For this, male mice were exposed to MCS (50\% of smoke of $12 \mathrm{KY}$ Reference 3R4F cigarettes, 6h), and urine was collected immediately after this single exposure session. Urinary levels of formate $(\mathrm{p}=0.01)$, 3HPMA ( $\mathrm{p}=0.01)$, HPMMA $(\mathrm{p}<0.001)$, and total nicotine metabolites $(\mathrm{p}<0.001)$ measured within the first 3 hours postexposure significantly exceeded levels measured after a 6h exposure to HEPA (Fig. 4.1). Levels of formate and acetate after the overnight (3-16h) collection were elevated compared with levels 0 -3h post-exposure; these elevations are related to generation by food, as mice were given free access to food during this collection period (Fig. 4.1).

\section{Systemic Toxicity of Acute Exposure to Inhaled MCS}

Tobacco use is responsible for an estimated 7 million global deaths each year (398), with many of these deaths due to CVD (399). Tobacco smoke contains thousands of compounds, including many unidentified compounds, many of which can contribute to CVD development even in very small quantities $(82,396)$. We therefore tested for changes in systemic and hematological biomarkers in mice exposed to MCS (50\% of smoke of 12 KY Reference 3R4F cigarettes). Acute (6h/day; 4d) exposure had modest effects on organ and body weights (or ratios) and CBCs. MCS exposure caused a lower final body weight $(-9 \% ; \mathrm{p}=0.003)$, liver/body weight ratio $(-17 \% ; \mathrm{p}=0.02)$, and, modestly, heart/body weight ratio $(-8 \% ; \mathrm{p}=0.06)$ (Table 4.1) than exposure to air. The overall leukocyte count was 


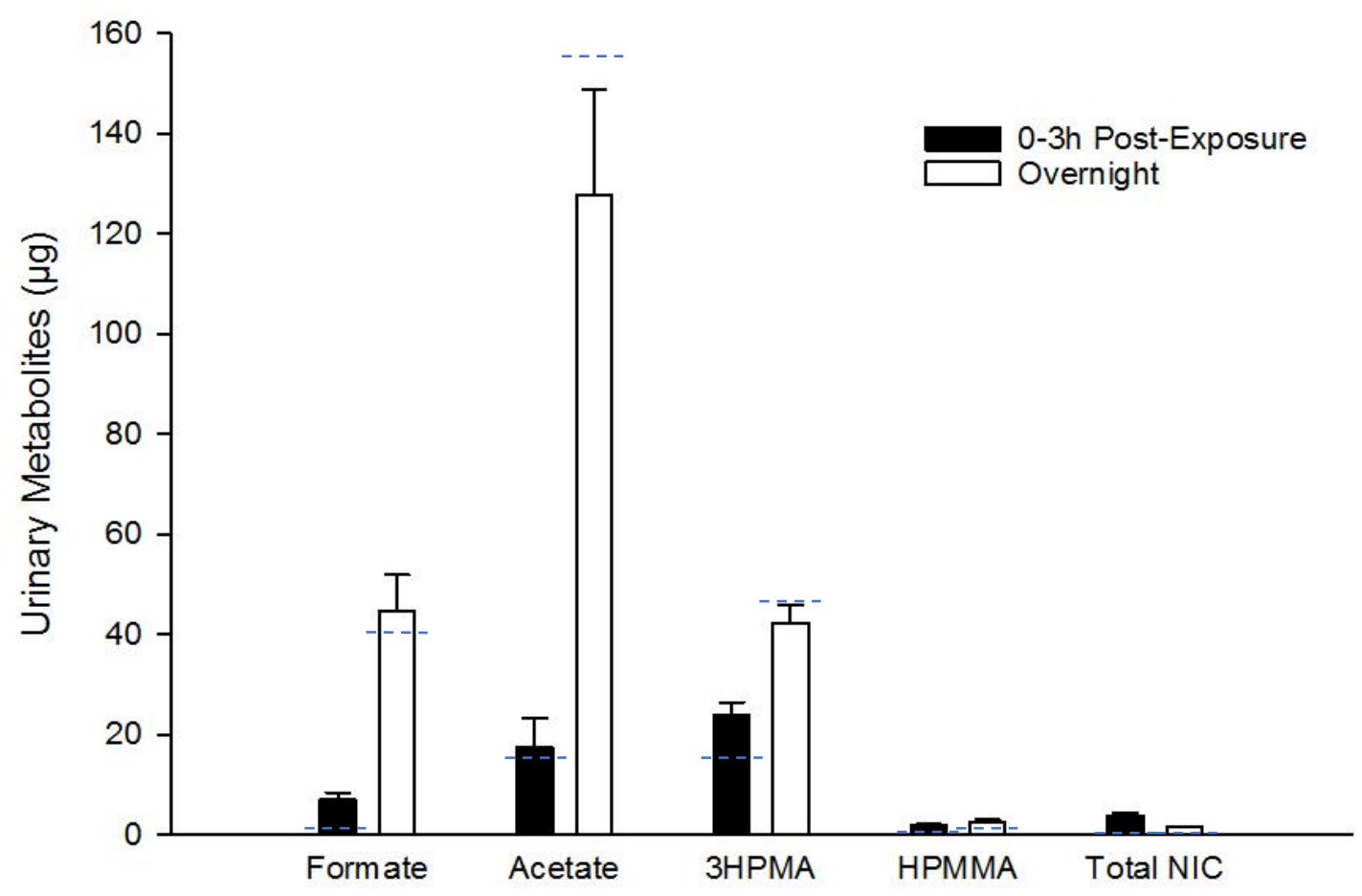

Fig. 4.1. Urinary metabolites after acute mainstream cigarette smoke (MCS) exposure. Male mice were exposed (6h) to mainstream cigarette smoke (MCS; 50\% of smoke of $12 \mathrm{KY}$ Reference 3R4F cigarettes) after which urine was collected at $0-1,1-2$, 2-3, and 3-16h (overnight) and aldehyde and tobacco alkaloid metabolites quantified. Values are mean $\pm S E$ ( $n=12$ mice per group). Dashed lines indicate metabolite measures ( $\mu \mathrm{g})$ after HEPA exposure (6h). Abbr.: 3HPMA, 3-hydroxypropylmercapturic acid; HPMMA, 3-hydroxy-1-methylpropylmercapturic acid; Total NIC, total urinary nicotine metabolites (nicotine, cotinine, and trans-3'-hydroxycotinine). 
Table 4.1. Systemic parameters of male C57BL/6 mice acutely exposed to either air or mainstream cigarette smoke (MCS; 50\% of smoke of $12 \mathrm{KY}$ Reference 3R4F cigarettes).

\begin{tabular}{|c|c|c|}
\hline \multirow[b]{2}{*}{ Variable } & \multicolumn{2}{|c|}{ Exposure (4 days) } \\
\hline & Air Control & MCS \\
\hline Final BWT (g) & $30 \pm 0$ & $27 \pm 0 *$ \\
\hline Heart/BWT (mg/g) & $5.5 \pm 0.2$ & $5.0 \pm 0.1 \#$ \\
\hline Lung/BWT (mg/g) & $5.9 \pm 0.2$ & $5.7 \pm 0.3$ \\
\hline Liver/BWT (mg/g) & $48.4 \pm 2.6$ & $40.4 \pm 0.7 *$ \\
\hline Kidney/BWT (mg/g) & $13.9 \pm 0.4$ & $13.3 \pm 0.1$ \\
\hline \multicolumn{3}{|c|}{ Plasma Measurements } \\
\hline Cholesterol (mg/dL) & $102.97 \pm 2.56$ & $100.25 \pm 3.72$ \\
\hline Triglycerides (mg/dL) & $28.75 \pm 6.19$ & $25.91 \pm 2.11$ \\
\hline Albumin (g/dL) & $3.53 \pm 0.17$ & $3.96 \pm 0.04 *$ \\
\hline Total Protein (g/dL) & $4.88 \pm 0.19$ & $4.94 \pm 0.05$ \\
\hline ALT (U/I) & $21.55 \pm 1.19$ & $22.63 \pm 0.65$ \\
\hline AST (U/I) & $52.67 \pm 5.41$ & $62.93 \pm 6.89$ \\
\hline CK (U/I) & $251.56 \pm 45.78$ & $234.16 \pm 31.19$ \\
\hline LDH (U/I) & $185.12 \pm 5.76$ & $187.65 \pm 9.32$ \\
\hline Creatinine (mg/dL) & $0.49 \pm 0.10$ & $0.35 \pm 0.01$ \\
\hline
\end{tabular}

Values = mean \pm SE ( $n=4-7$ mice per group $)$; Creatinine compared using Rank Sum Test. Abbr.: ALT, alanine aminotransferase; AST, aspartate aminotransferase; BWT, body weight; CK, creatine kinase; LDH, lactate dehydrogenase; MCS, mainstream cigarette smoke;

*, $\mathrm{p}<0.05$ compared with air control based on Bonferroni's or Dunn's post-test; \#, $0.05 \leq \mathrm{p} \leq 0.10$ compared with air control based on Bonferroni's or Dunn's post-test. 
significantly decreased with exposure $(-60 \% ; \mathrm{p}=0.01)$, driven by a decrease in lymphocytes $(-58 \% ; \mathrm{p}=0.01)$, as was mean corpuscular volume $(-10 \% ; \mathrm{p}=0.002)$, while mean corpuscular hemoglobin concentration was increased (+11\%; p<0.001) (Table 4.2). Plasma levels of liver (ALT, AST), muscle (CK), and LDH enzymes and creatinine were unaffected by exposure, but albumin was significantly increased $(+12 \%$; $\mathrm{p}=0.004)$ (Table 4.1).

\section{Acute Exposure to MCS and Circulating Angiogenic Cells}

Acute (4d) exposure to MCS (50\% of smoke of $12 \mathrm{KY}$ Reference 3R4F cigarettes) caused a significant decrease in levels of Sca-1+ cells $(-56 \%$; p=0.03) (Fig. 4.2A) but did not change the levels of CACs (Fig. 4.2B).

\section{Urinary Metabolites in JUUL E-Cig-Exposed Mice}

The use of e-cig devices has been increasing dramatically in both adolescents and adults over the past several years $(83,181-183,325)$. Although e-cigs are commonly touted as a healthier alternative to traditional cigarettes $(173-175,177,181,184)$, the health effects of these devices are still largely unknown. Furthermore, a number of studies have detected harmful constituents including aldehydes in the aerosol of these devices, which may indicate that e-cigs can contribute to adverse health risks in a fashion similar to traditional cigarettes $(96,180,207,211)$. To better characterize exposure to e-cig aerosol, we measured urinary formate, acetate, 3HPMA, and total urinary nicotine metabolites (nicotine, cotinine, and 3HC) by GC-MS and UPLC-MS/MS to evaluate the presence of aldehydes in aerosols generated by e-cig devices. For this, male mice were exposed to aerosol from flavored JUUL e-cig devices (Virginia (VA) Tobacco, Mango, or Menthol; 
Table 4.2. Hematological measures of male C57BL/6 mice acutely exposed to either air or mainstream cigarette smoke (MCS; 50\% of smoke of $12 \mathrm{KY}$ Reference 3R4F cigarettes).

\begin{tabular}{|l|c|c|}
\hline \multicolumn{1}{|c|}{ Hematological Measurements } & \multicolumn{2}{c|}{ Exposure (4 days) } \\
\hline White Blood Cell (K/uL) & Air Control & MCS \\
\hline Neutrophils (K/uL) & $1.69 \pm 0.21$ & $0.68 \pm 0.09^{*}$ \\
\hline Lymphocytes (K/uL) & $0.47 \pm 0.09$ & $0.15 \pm 0.02$ \\
\hline Monocytes (K/uL) & $1.17 \pm 0.17$ & $0.50 \pm 0.07^{*}$ \\
\hline Eosinophils (K/uL) & $0.05 \pm 0.01$ & $0.03 \pm 0.01$ \\
\hline Basophils (K/uL) & $0 \pm 0$ & $0 \pm 0$ \\
\hline Red Blood Cell (M/uL) & $0 \pm 0$ & $0 \pm 0$ \\
\hline Hemoglobin (g/dL) & $8.31 \pm 0.14$ & $8.78 \pm 0.14$ \\
\hline Hematocrit (\%) & $11.6 \pm 0.5$ & $12.1 \pm 0.1$ \\
\hline Mean Corpuscular Volume (fL) & $39.0 \pm 1.8$ & $36.8 \pm 0.6$ \\
\hline Mean Corpuscular Hemoglobin (pg) & $46.8 \pm 1.4$ & $41.9 \pm 0.6^{*}$ \\
\hline Mean Corpuscular Hemoglobin Concentration (g/dL) & $13.9 \pm 0.4$ & $13.8 \pm 0.1$ \\
\hline Red Cell Distribution Width (\%) & $29.7 \pm 0.1$ & $32.9 \pm 0.3^{*}$ \\
\hline Platelets (K/uL) & $17.6 \pm 0.2$ & $17.9 \pm 0.2$ \\
\hline Mean Platelet Volume (fL) & $912 \pm 105$ & $1049 \pm 28$ \\
\hline
\end{tabular}

Values $=$ mean \pm SE ( $\mathrm{n}=4-5$ mice per group $)$; Abbr.: MCS, mainstream cigarette smoke; $*, \mathrm{p}<0.05$ compared with air control based on Bonferroni's or Dunn's post-test. 
A.
B.

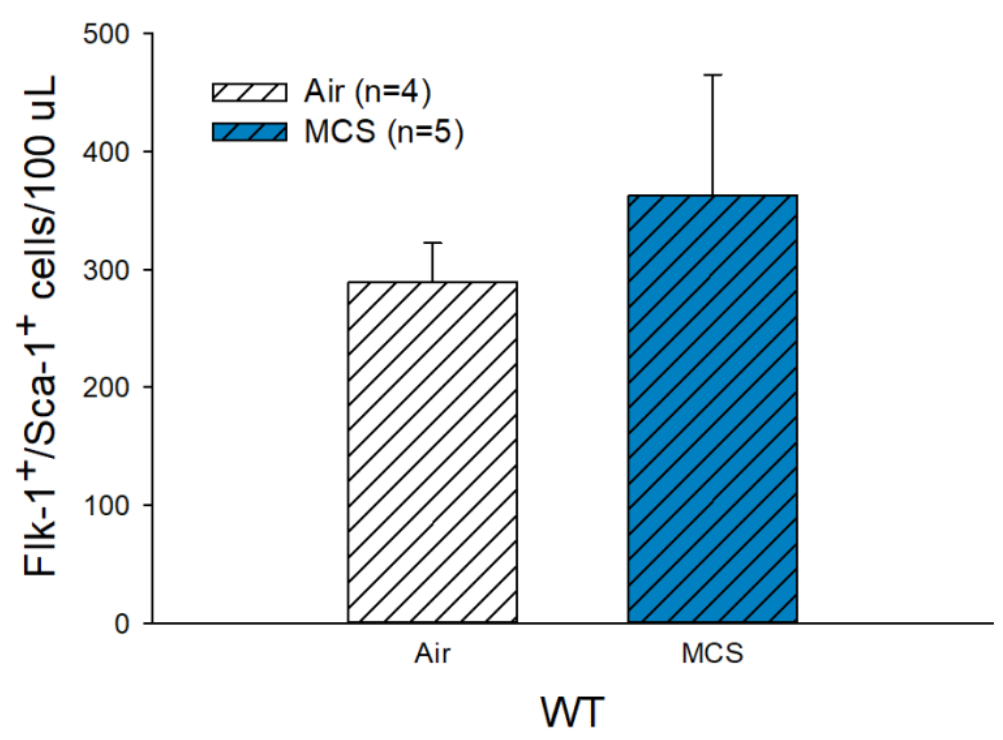

Fig. 4.2. Effects of acute mainstream cigarette smoke (MCS) exposure on circulating stem cells in male mice. Hematopoietic stem cells (Sca-1+) and circulating angiogenic cells (CACs) were measured by flow cytometry in male C57BL/6J (wild type, WT) mice exposed to HEPA-filtered air or MCS (50\% of smoke of 12 KY Reference 3R4F Cigarettes, 6h/d) for 4 days. After 4 days of exposure to MCS, the level of circulating Sca-1+ cells was significantly decreased in WT mice (A), however, no change in the level of CACs $($ Flk-1+/Sca- $1+$ cells $)$ was detected relative to air-exposed control mice $(\mathbf{B})$. Values $=$ mean $\pm \mathrm{SE}(\mathrm{n}=4-5$ mice per group). $*, \mathrm{p}<0.05$ compared with air control based on Bonferroni's or Dunn's post-test. 
6h), and urine was collected immediately after the exposure sessions. Although acrolein has been measured in e-cig aerosol, urinary levels of 3HPMA after the JUUL exposures were not significantly different from levels after acute air exposure (6h) (Figs. 4.3, 4.4, \& 4.5). Likewise, levels of urinary formate and acetate were not significantly different from levels in air-exposed mice (Figs. 4.3, 4.4, \& 4.5). Measures of total urinary nicotine metabolites, however, were significantly elevated post-JUUL exposure (VA Tobacco: $\mathrm{p}<0.001$; Mango and Menthol: $\mathrm{p}=0.002$ ); mice exposed to either JUUL Mango or JUUL Menthol showed similar levels of urinary nicotine $(\mathrm{p}=0.2)$, which were significantly higher than levels in mice exposed to JUUL VA Tobacco ( $\mathrm{p}=0.02$ for both) (Figs. 4.3, 4.4, \& 4.5). Levels of formate and acetate after the overnight (3-16h) collection were elevated compared with levels $0-3 \mathrm{~h}$ post-exposure for exposure to all three JUUL products; these elevations are likely related to generation by food, as mice were given free access to food during this collection period (Figs. 4.3, 4.4, \& 4.5).

\section{Systemic Toxicity of Acute Exposure to Inhaled JUUL Mango E-cig Aerosol}

Because of the known health effects of aldehydes found to be generated in the aerosol of e-cigarette (e-cig) devices $(96,180,207-213)$ and because of the potential risks of flavorings used in these devices $(183,218,220-223)$, we tested for changes in systemic and hematological biomarkers in female mice exposed to aerosol from a JUUL e-cig device with mango flavoring (JUUL Mango). Acute (6h/day; 4d) exposure had a number of significant effects on organ and body weights (or ratios) and $\mathrm{CBC}$. For example, exposure to JUUL Mango caused a greater change in body weight compared with air-exposed control mice $(-3 \mathrm{~g}$ vs $-2 \mathrm{~g} ; \mathrm{p}=0.01)$, with a modest increase in heart/body weight ratio $(+6 \%$; 


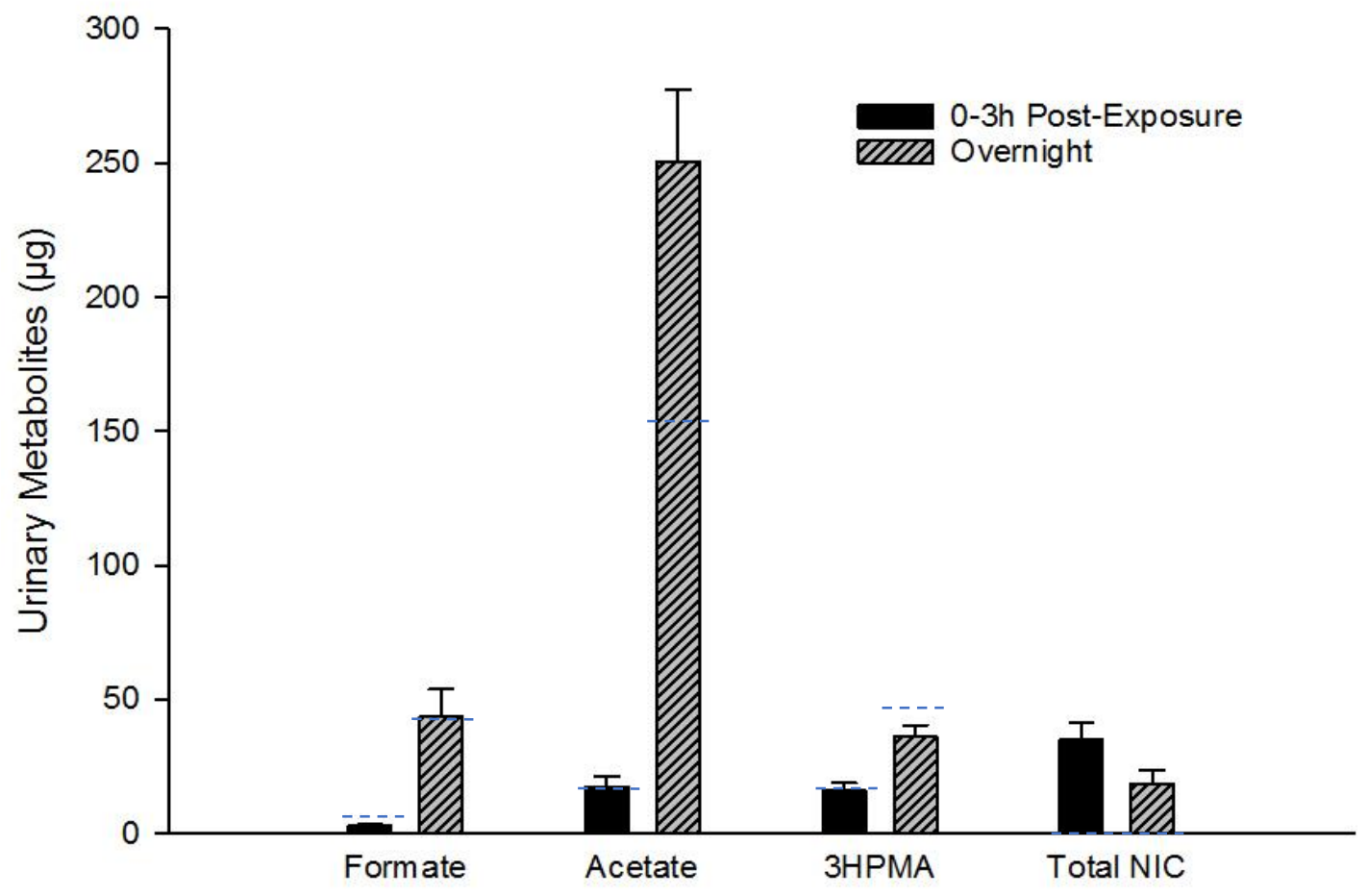

Fig. 4.3. Urinary metabolites after acute electronic cigarette aerosol (e-cig; JUUL VA Tobacco) exposure. Male mice were exposed (6h) to e-cig aerosol (JUUL VA Tobacco) after which urine was collected at $0-1,1-2,2-3$, and 3-16h (overnight) and aldehyde and tobacco alkaloid metabolites quantified. Values are mean $\pm \mathrm{SE}$ ( $\mathrm{n}=5$ mice per group). Dashed lines indicate metabolite measures $(\mu \mathrm{g})$ after HEPA exposure (6h). Abbr.: 3HPMA, 3-hydroxypropylmercapturic acid; Total NIC, total urinary nicotine metabolites (nicotine, cotinine, and trans-3'-hydroxycotinine). 


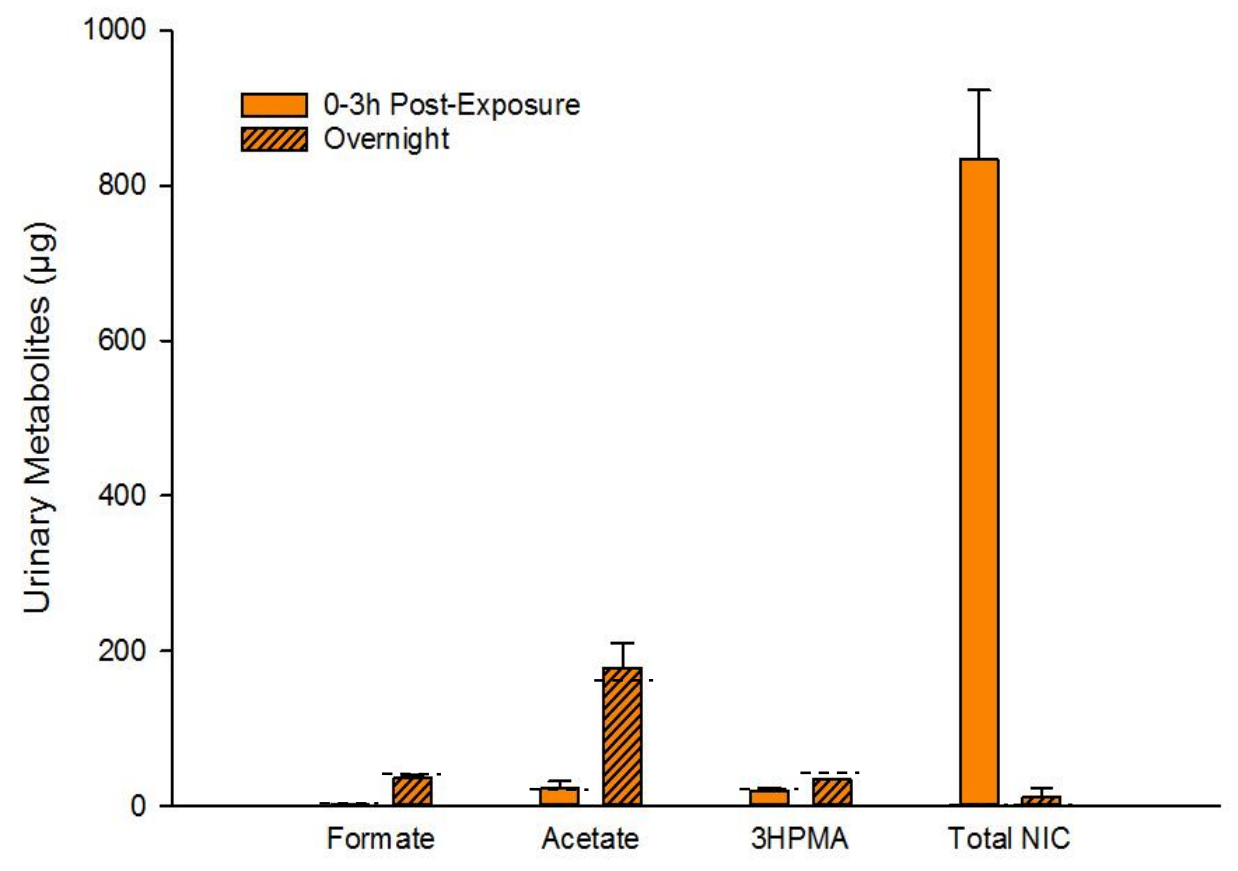

Fig. 4.4. Urinary metabolites after acute electronic cigarette aerosol (e-cig; JUUL Mango) exposure. Male mice were exposed (6h) to e-cig aerosol (JUUL VA Mango) after which urine was collected at $0-1,1-2,2-3$, and 3-16h (overnight) and aldehyde and tobacco alkaloid metabolites quantified. Values are mean $\pm \mathrm{SE}$ ( $\mathrm{n}=4$ mice per group). Dashed lines indicate metabolite measures $(\mu \mathrm{g})$ after HEPA exposure (6h). Abbr.: 3HPMA, 3-hydroxypropylmercapturic acid; Total NIC, total urinary nicotine metabolites (nicotine, cotinine, and trans-3'-hydroxycotinine). 


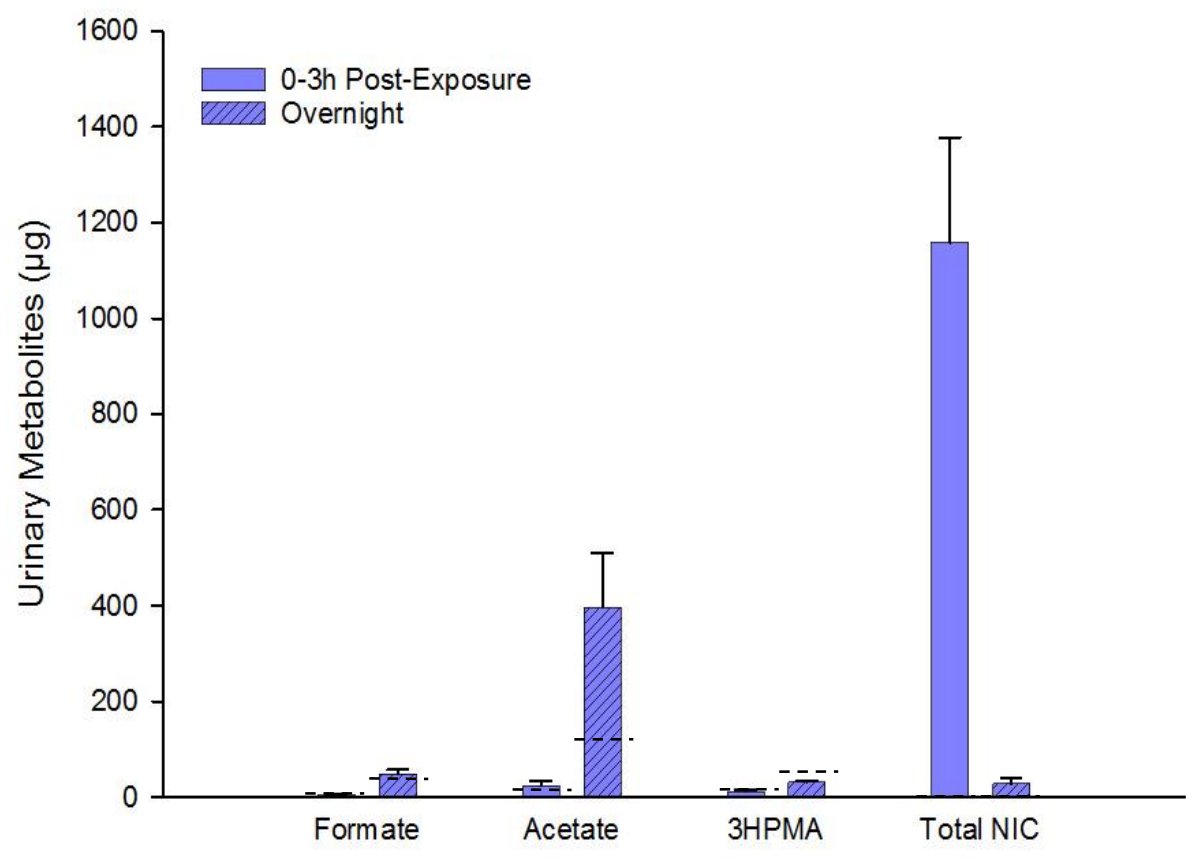

Fig. 4.5. Urinary metabolites after acute electronic cigarette aerosol (e-cig; JUUL Menthol) exposure. Male mice were exposed (6h) to e-cig aerosol (JUUL Menthol) after which urine was collected at $0-1,1-2,2-3$, and 3-16h (overnight) and aldehyde and tobacco alkaloid metabolites quantified. Values are mean $\pm \mathrm{SE}$ ( $\mathrm{n}=4$ mice per group). Dashed lines indicate metabolite measures $(\mu \mathrm{g})$ after HEPA exposure (6h). Abbr.: 3HPMA, 3-hydroxypropylmercapturic acid; Total NIC, total urinary nicotine metabolites (nicotine, cotinine, and trans-3'-hydroxycotinine). 
$\mathrm{p}=0.06)$ and a significant increase in kidney/body weight ratio $(+7 \% ; \mathrm{p}=0.045)$ (Table 4.3).

The overall leukocyte count was significantly decreased $(-48 \% ; \mathrm{p}=0.001)$, driven by decreases in neutrophils $(-54 \% ; \mathrm{p}=0.004)$, lymphocytes $(-46 \% ; \mathrm{p}=0.002)$, and monocytes $(-48 \% ; \mathrm{p}=0.06)$ (Table 4.4). However, changes in erythrocytic measures were only seen in hemoglobin $(+3 \% ; \mathrm{p}=0.02)$ and hematocrit $(+4 \% ; \mathrm{p}=0.05)$ (Table 4.4). Although plasma levels of muscle (CK) and LDH enzyme and creatinine were unchanged in exposed mice, exposure caused modestly significant increases in both albumin $(+8 \% ; \mathrm{p}=0.06)$ and AST $(+34 \% ; \mathrm{p}=0.06)($ Table 4.3$)$.

\section{Acute JUUL Mango E-cig Aerosol Exposure and CACs}

Acute (4d) exposure to JUUL Mango aerosol caused modest decreases in levels of both Sca- $1+$ cells $(-67 \% ; \mathrm{p}=0.06)$ and CACs $(-75 \% ; \mathrm{p}=0.06)($ Fig. 4.6A \& B).

\section{Systemic Toxicity of Acute Exposure to blu Plus+ E-cig Aerosol}

E-cig aerosol has been linked to negative health effects (174, 190-192), but the mechanism of these effects has not been identified. Thus, we tested for changes in systemic and hematological biomarkers in mice exposed to e-cig (blu Plus+) aerosol (4d). Acutelyexposed male mice showed a significantly decreased final body weight $(-6 \% ; \mathrm{p}=0.003)$ but no changes in measured organ/body weight ratios (Table 4.5). Although plasma levels of liver (ALT, AST), muscle (CK), and LDH enzymes and creatinine were unaffected by exposure, triglycerides were modestly decreased $(-12 \% ; \mathrm{p}=0.09)$ (Table 4.5). Measures of toxicity by $\mathrm{CBC}$ showed considerable effects. For example, e-cig exposure significantly decreased leukocyte count $(-36 \% ; \mathrm{p}<0.001)$, driven by decreases in all leukocyte subpopulations (Table 4.6). Increases in mean corpuscular hemoglobin $(+7 \%$; $p<0.001)$, mean corpuscular hemoglobin concentration $(+6 \%$; $\mathrm{p}<0.001)$, and, modestly, hemoglobin 
Table 4.3. Systemic parameters of female C57BL/6 mice acutely exposed to either air or electronic cigarette aerosol (e-cig; JUUL

Mango).

\begin{tabular}{|c|c|c|}
\hline \multirow[b]{2}{*}{ Variable } & \multicolumn{2}{|c|}{ Exposure (4 days) } \\
\hline & Air Control & JUUL Mango \\
\hline Change in BWT (g) & $-2 \pm 0$ & $-3 \pm 0 *$ \\
\hline Heart/BWT (mg/g) & $5.0 \pm 0.1$ & $5.3 \pm 0.1 \#$ \\
\hline Lung/BWT (mg/g) & $6.5 \pm 0.2$ & $6.6 \pm 0.2$ \\
\hline Liver/BWT (mg/g) & $42.9 \pm 0.7$ & $43.5 \pm 1.0$ \\
\hline Kidney/BWT (mg/g) & $12.7 \pm 0.2$ & $13.7 \pm 0.3^{*}$ \\
\hline \multicolumn{3}{|c|}{ Plasma Measurements } \\
\hline Cholesterol (mg/dL) & $59.40 \pm 1.12$ & $56.20 \pm 1.77$ \\
\hline HDL (mg/dL) & $30.40 \pm 0.93$ & $29.20 \pm 1.24$ \\
\hline LDL (mg/dL) & $21.44 \pm 0.42$ & $20.20 \pm 1.10$ \\
\hline Triglycerides (mg/dL) & $37.80 \pm 0.92$ & $34.00 \pm 3.61$ \\
\hline Albumin (g/dL) & $2.68 \pm 0.07$ & $2.90 \pm 0.05 \#$ \\
\hline Total Protein (g/dL) & $4.46 \pm 0.09$ & $4.66 \pm 0.09$ \\
\hline ALT (U/I) & $7.20 \pm 0.92$ & $7.60 \pm 0.60$ \\
\hline AST (U/I) & $35.75 \pm 2.63$ & $47.80 \pm 4.42 \#$ \\
\hline CK (U/I) & $105.25 \pm 7.12$ & $175.60 \pm 47.54$ \\
\hline LDH (U/I) & $120.20 \pm 19.34$ & $117.80 \pm 6.15$ \\
\hline Creatinine (mg/dL) & $0.19 \pm 0.01$ & $0.19 \pm 0.01$ \\
\hline
\end{tabular}

Values = mean \pm SE (n=5-10 mice per group); Abbr.: ALT, alanine aminotransferase; AST, aspartate aminotransferase; BWT, body weight; CK, creatine kinase; E-cig, electronic cigarette; HDL, high-density lipoprotein; LDH, lactate dehydrogenase; LDL, low-density lipoprotein; *, $\mathrm{p}<0.05$ compared with air control; \#, $0.05 \leq \mathrm{p} \leq 0.10$ compared with air control. 
Table 4.4. Hematological measures of female C57BL/6 mice acutely exposed to either air or electronic cigarette aerosol (e-cig; JUUL

Mango).

\begin{tabular}{|l|c|c|}
\hline \multicolumn{1}{|c|}{ Hematological Measurements } & \multicolumn{2}{c|}{ Exposure (4 days) } \\
\hline White Blood Cell (K/uL) & Air Control & JUUL Mango \\
\hline Neutrophils (K/uL) & $1.15 \pm 0.13$ & $0.60 \pm 0.07^{*}$ \\
\hline Lymphocytes (K/uL) & $0.23 \pm 0.03$ & $0.11 \pm 0.01^{*}$ \\
\hline Monocytes (K/uL) & $0.88 \pm 0.10$ & $0.48 \pm 0.06^{*}$ \\
\hline Eosinophils (K/uL) & $0.03 \pm 0.01$ & $0.02 \pm 0 \#$ \\
\hline Basophils (K/uL) & $0 \pm 0$ & $0 \pm 0$ \\
\hline Red Blood Cell (M/uL) & $0 \pm 0$ & $0 \pm 0$ \\
\hline Hemoglobin (g/dL) & $9.07 \pm 0.08$ & $9.33 \pm 0.14$ \\
\hline Hematocrit (\%) & $12.6 \pm 0.1$ & $13.0 \pm 0.1^{*}$ \\
\hline Mean Corpuscular Volume (fL) & $46.9 \pm 0.5$ & $48.6 \pm 0.5 \#$ \\
\hline Mean Corpuscular Hemoglobin (pg) & $51.7 \pm 0.3$ & $51.5 \pm 0.2$ \\
\hline Mean Corpuscular Hemoglobin Concentration (g/dL) & $13.8 \pm 0.1$ & $13.8 \pm 0.1$ \\
\hline Red Cell Distribution Width (\%) & $26.8 \pm 0.1$ & $26.7 \pm 0.2$ \\
\hline Platelets (K/uL) & $16.3 \pm 0.1$ & $16.2 \pm 0.1$ \\
\hline Mean Platelet Volume (fL) & $723 \pm 15$ & $694 \pm 24$ \\
\hline
\end{tabular}

Values $=$ mean $\pm \mathrm{SE}(\mathrm{n}=10$ mice per group $) ;$ Abbr.: E-cig, electronic cigarette; ${ }^{*}, \mathrm{p}<0.05$ compared with air control; \#, $0.05 \leq \mathrm{p} \leq 0.10$ compared with air control. 
A.

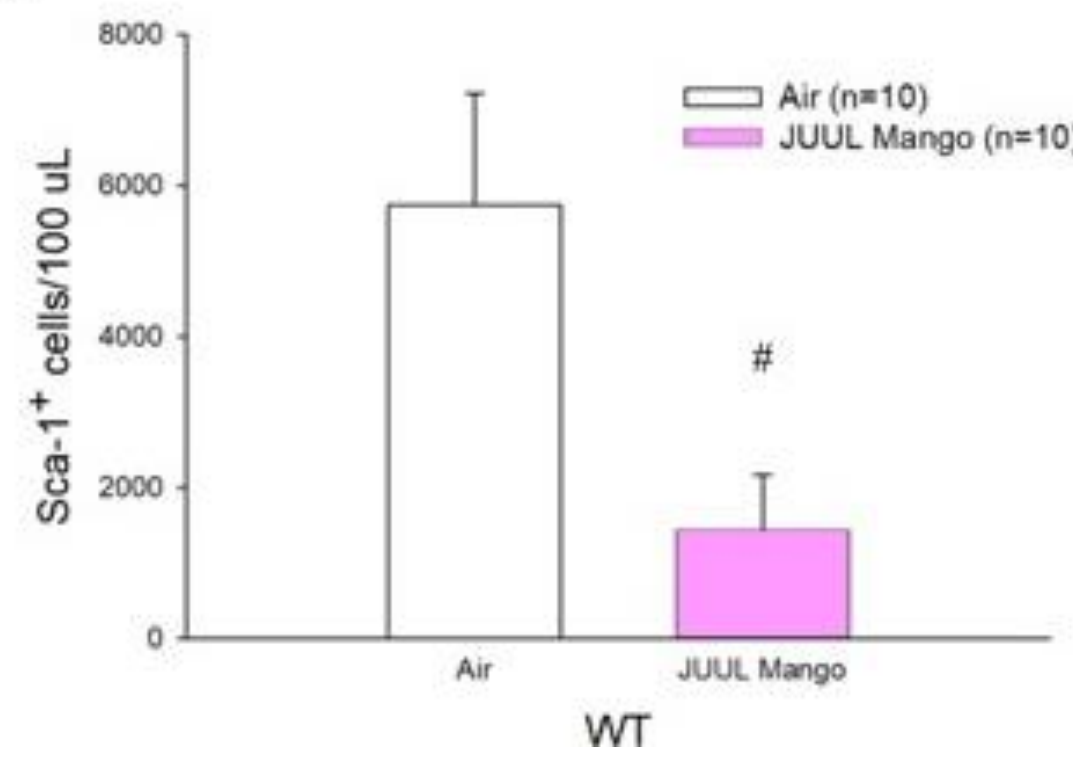

B.

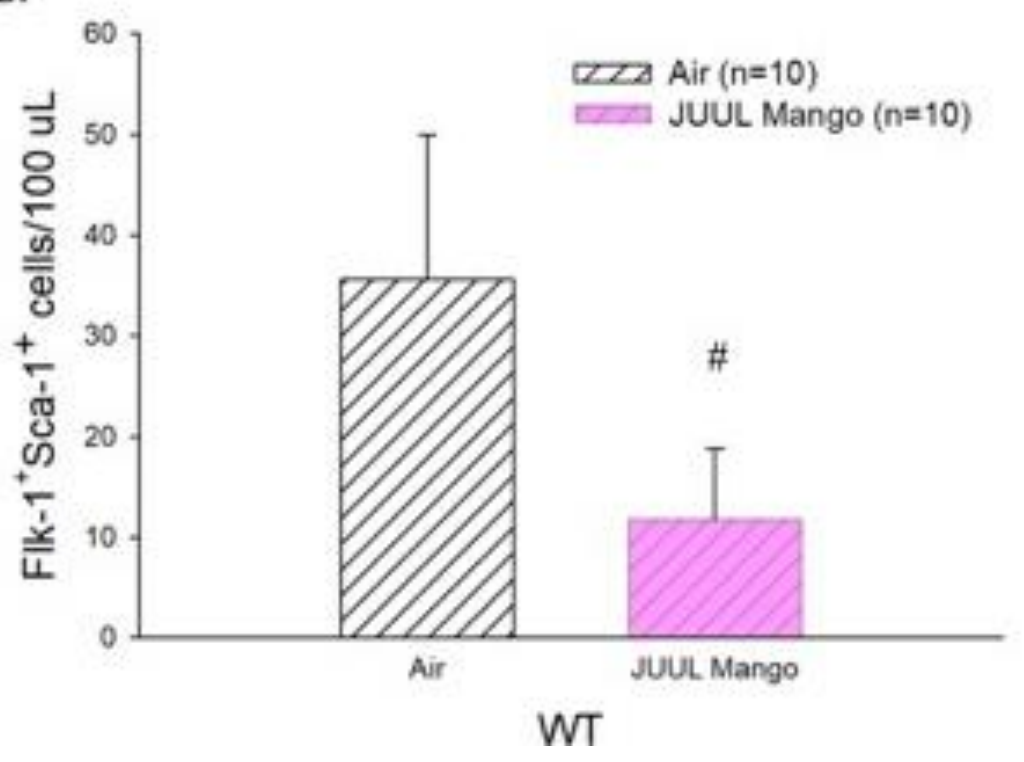

Fig. 4.6. Effects of acute electronic cigarette (e-cig; JUUL Mango) aerosol exposure on circulating stem cells in female mice.

Hematopoietic stem cells (Sca-1+) and circulating angiogenic cells (CACs) were measured by flow cytometry in female C57BL/6J (wild type, WT) mice exposed to HEPA-filtered air or JUUL Mango e-cigarette (e-cig) aerosol (6h/d) for 4 days. After 4 days of exposure to JUUL Mango, the level of both circulating Sca-1+ cells (A) and the level of CACs (Flk-1+/Sca-1+ cells) (B) decreased relative to airexposed control mice. Values $=$ mean $\pm \mathrm{SE}(\mathrm{n}=5$ mice per group). \#, $0.05 \leq \mathrm{p} \leq 0.10$ compared with air control. 
Table 4.5. Systemic parameters of male C57BL/6 mice acutely exposed to either air or electronic cigarette aerosol (e-cig; blu Plus+).

\begin{tabular}{|c|c|c|}
\hline \multirow[b]{2}{*}{ Variable } & \multicolumn{2}{|c|}{ Exposure (4 days) } \\
\hline & Air Control & blu Plus+e-cig \\
\hline Final BWT (g) & $27 \pm 0$ & $25 \pm 0 *$ \\
\hline Heart/BWT (mg/g) & $5.1 \pm 0.1$ & $5.2 \pm 0.1$ \\
\hline Lung/BWT (mg/g) & $5.6 \pm 0.1$ & $5.8 \pm 0.2$ \\
\hline Liver/BWT (mg/g) & $45.0 \pm 0.8$ & $44.3 \pm 0.8$ \\
\hline Kidney/BWT (mg/g) & $12.1 \pm 0.3$ & $12.3 \pm 0.2$ \\
\hline \multicolumn{3}{|c|}{ Plasma Measurements } \\
\hline Cholesterol (mg/dL) & $83.25 \pm 2.53$ & $78.45 \pm 2.78$ \\
\hline HDL (mg/dL) & $49.57 \pm 1.04$ & $44.85 \pm 5.50$ \\
\hline LDL (mg/dL) & $5.84 \pm 0.42$ & $6.09 \pm 1.07$ \\
\hline Triglycerides (mg/dL) & $44.19 \pm 2.09$ & $39.01 \pm 1.69_{\#}$ \\
\hline Albumin (g/dL) & $2.86 \pm 0.04$ & $2.93 \pm 0.05$ \\
\hline Total Protein (g/dL) & $4.04 \pm 0.16$ & $3.89 \pm 0.16$ \\
\hline ALT (U/I) & $23.46 \pm 2.46$ & $20.18 \pm 2.08$ \\
\hline AST (U/I) & $53.03 \pm 6.73$ & $40.94 \pm 2.89$ \\
\hline CK (U/I) & $178.42 \pm 18.62$ & $157.50 \pm 11.00$ \\
\hline LDH (U/I) & $166.19 \pm 12.36$ & $155.11 \pm 11.36$ \\
\hline Creatinine (mg/dL) & $0.23 \pm 0.01$ & $0.24 \pm 0.01$ \\
\hline
\end{tabular}

Values = mean \pm SE (n=9-10 mice per group); Abbr.: ALT, alanine aminotransferase; AST, aspartate aminotransferase; BWT, body weight; CK, creatine kinase; E-cig, electronic cigarette; HDL, high-density lipoprotein; LDH, lactate dehydrogenase; LDL, low-density lipoprotein; *, $\mathrm{p}<0.05$ compared with air control; \#, $0.05 \leq \mathrm{p} \leq 0.10$ compared with air control. 
Table 4.6. Hematological measures of male C57BL/6 mice acutely exposed to either air or electronic cigarette aerosol (e-cig; blu Plus+).

\begin{tabular}{|l|c|c|}
\hline \multicolumn{1}{|c|}{ Hematological Measurements } & \multicolumn{2}{c|}{ Exposure (4 days) } \\
\hline \multicolumn{1}{|c|}{ bir Control } & blu Plus+ e-cig \\
\hline White Blood Cell (K/uL) & $1.80 \pm 0.16$ & $1.15 \pm 0.12^{*}$ \\
\hline Neutrophils (K/uL) & $0.45 \pm 0.07$ & $0.28 \pm 0.05^{*}$ \\
\hline Lymphocytes (K/uL) & $1.29 \pm 0.11$ & $0.85 \pm 0.08^{*}$ \\
\hline Monocytes (K/uL) & $0.06 \pm 0.01$ & $0.03 \pm 0^{*}$ \\
\hline Eosinophils (K/uL) & $0.01 \pm 0$ & $0 \pm 0^{*}$ \\
\hline Basophils (K/uL) & $0 \pm 0$ & $0 \pm 0$ \\
\hline Red Blood Cell (M/uL) & $7.66 \pm 0.29$ & $7.98 \pm 0.33$ \\
\hline Hemoglobin (g/dL) & $10.5 \pm 0.5$ & $11.7 \pm 0.6 *$ \\
\hline Hematocrit (\%) & $33.6 \pm 1.4$ & $35.1 \pm 1.6$ \\
\hline Mean Corpuscular Volume (fL) & $43.7 \pm 0.2$ & $42.3 \pm 1.7$ \\
\hline Mean Corpuscular Hemoglobin (pg) & $13.7 \pm 0.1$ & $14.6 \pm 0.4 *$ \\
\hline Mean Corpuscular Hemoglobin Concentration (g/dL) & $31.3 \pm 0.2$ & $33.3 \pm 0.4 *$ \\
\hline Red Cell Distribution Width (\%) & $16.9 \pm 0.2$ & $17.4 \pm 0.4$ \\
\hline Platelets (K/uL) & $592 \pm 21$ & $4.2 \pm 0$ \\
\hline Mean Platelet Volume (fL) & $605 \pm 30$ & $4.4 \pm 0.2$ \\
\hline
\end{tabular}

Values $=$ mean $\pm \mathrm{SE}(\mathrm{n}=9-10$ mice per group); Abbr.: E-cig, electronic cigarette; $*, \mathrm{p}<0.05$ compared with air control; \#, 0.05 $\leq \mathrm{p} \leq 0.10$ compared with air control. 
$(+11 \% ; \mathrm{p}=0.10)$ were also seen in e-cig-exposed mice compared with air-exposed control mice (Table 4.6).

\section{Acute blu Plus+ E-cig Aerosol Exposure and Circulating Angiogenic Cells}

Male mice acutely exposed to e-cig (blu Plus+) aerosol (4d) showed no changes in the levels of CACs or Sca-1+ cells (Fig. 4.7A \& B).

\section{Urinary Metabolites in PG:VG-Exposed Mice}

PG and VG are commonly used as humectants in e-cig devices (179). The ratio of these compounds can vary between different e-cig devices, as well as with user modification, and variations in these ratios may influence the generation of different compounds in the e-cig aerosol (179). To better characterize exposure to PG:VG in e-cig devices and to determine how varying ratios may impact aldehyde production, we measured urinary formate, acetate, 3HPMA, HPMMA, and/or total urinary nicotine metabolites by GC-MS and UPLC-MS/MS to evaluate the presence of PG:VG-related aldehydes in aerosols generated by e-cig devices. For this, male mice were exposed to PG:VG (50\%:50\% or 30\%:70\%, 6h), and urine was collected immediately after exposure. Urinary levels of formate during the first three hours post-exposure were significantly increased in mice exposed to 50\%:50\% PG:VG compared with control mice $(\mathrm{p}=0.003)$ (Fig. 4.8), while urinary acetate was increased with exposure to 30\%:70\% PG:VG $(\mathrm{p}=0.047)$ (Fig. 4.9). Similarly, the first three hours post-exposure showed significantly increased levels of urinary 3HPMA after exposure to either ratio of $\mathrm{PG}: \mathrm{VG}(\mathrm{p}<0.001$ for 50\%50\% PG:VG; p=0.02 for 30\%:70\% PG:VG) (Figs. $4.8 \& 4.9$ ). Mice exposed to 50\%:50\% PG:VG also showed a small but measurable level of HPMMA, the major urinary metabolite of crotonaldehyde, although the measured level was not significantly different 
A.
B.

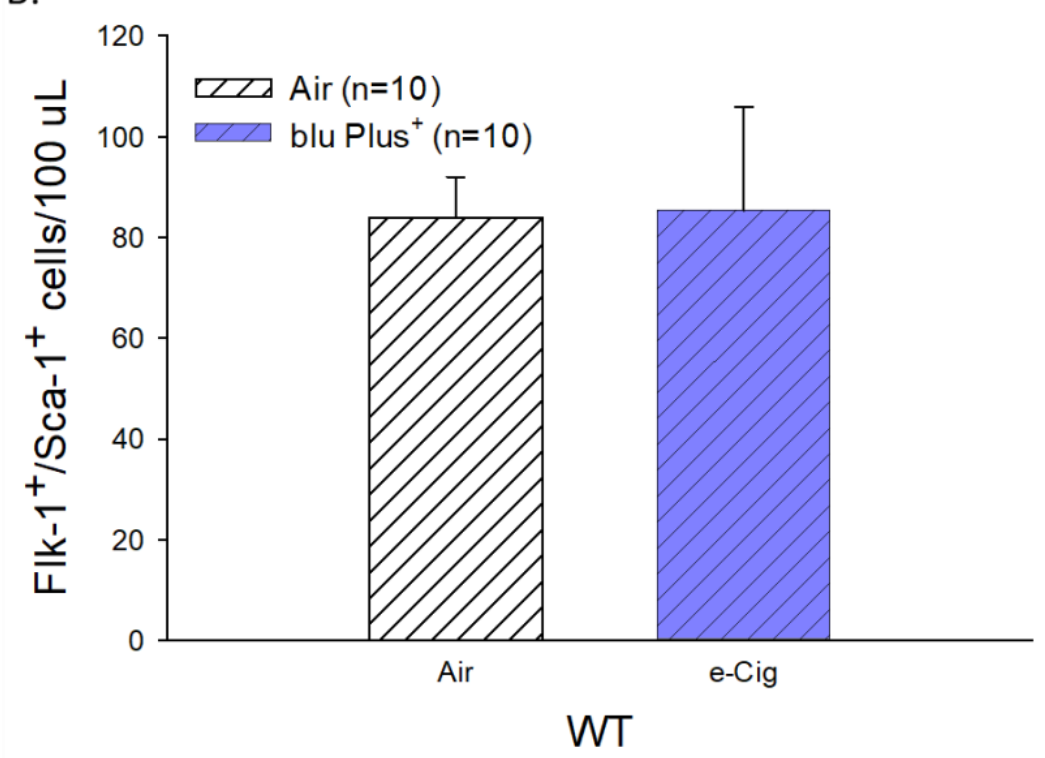

Fig. 4.7. Effects of acute electronic cigarette (e-cig; blu Plus+) aerosol exposure on circulating stem cells in male mice. Hematopoietic stem cells (Sca-1+) and circulating angiogenic cells (CACs) were measured by flow cytometry in male C57BL/6J (wild type, WT) mice exposed to HEPA-filtered air or e-cig aerosol (blu Plus+, 6h/d) for 4 days. Levels of circulating Sca-1+ cells (A) and CACs $($ Flk-1+/Sca-1+ cells; $\mathbf{B})$ were unchanged in e-cig aerosol-exposed mice relative to air control mice. Values $=$ mean $\pm \mathrm{SE}(\mathrm{n}=10$ mice per group). 


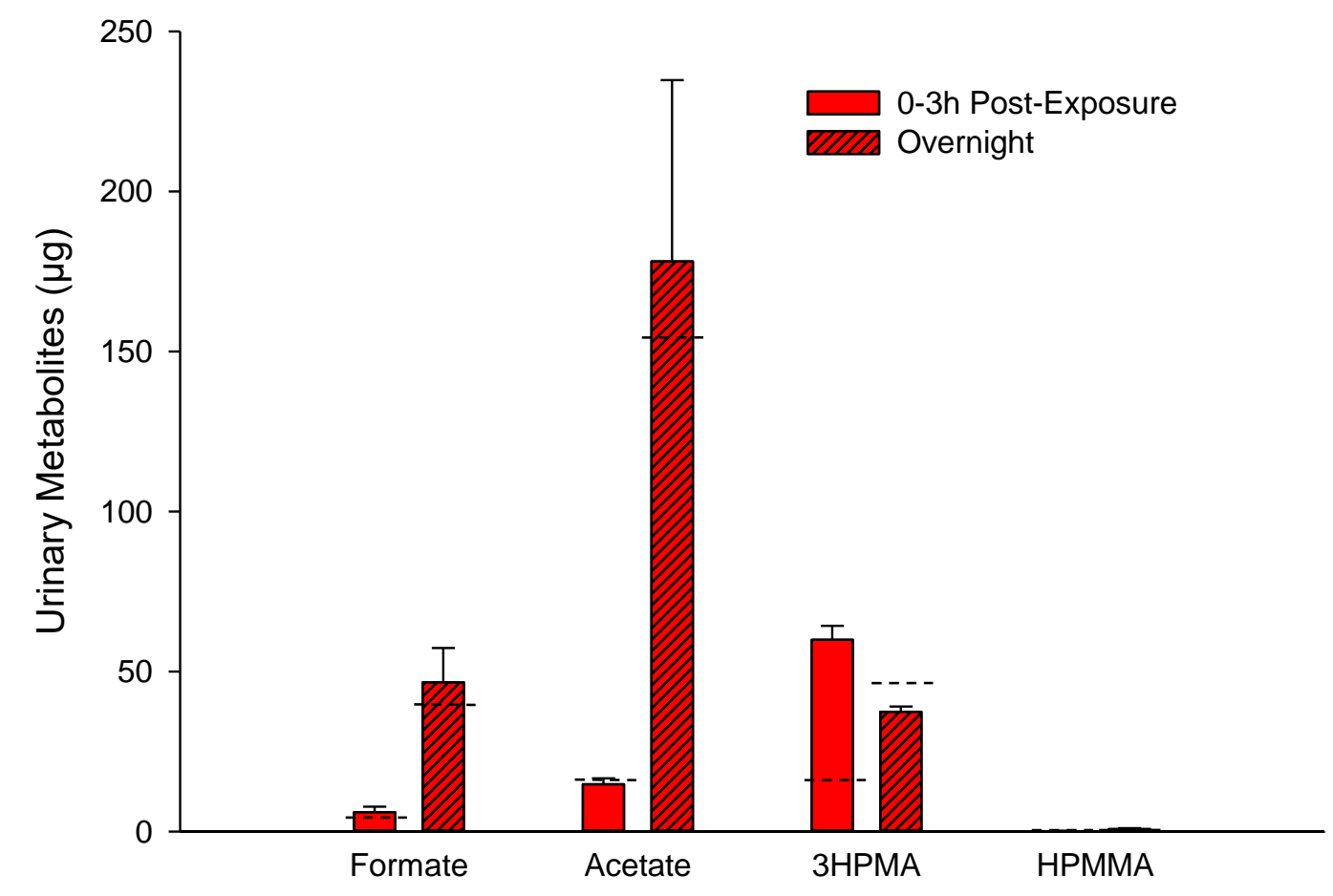

Fig. 4.8. Urinary metabolites after acute propylene glycol:vegetable glycerin (PG:VG; 50\%:50\%) exposure. Male mice were exposed (6h) to PG:VG (50\%:50\%) after which urine was collected at $0-1,1-2,2-3$, and $3-16 \mathrm{~h}$ (overnight) and aldehyde metabolites quantified. Values are mean $\pm \mathrm{SE}$ ( $\mathrm{n}=5$ mice per group). Dashed lines indicate metabolite measures $(\mu \mathrm{g})$ after HEPA exposure (6h). Abbr.: 3HPMA, 3-hydroxypropylmercapturic acid; PG:VG, propylene glycol:vegetable glycerin. 


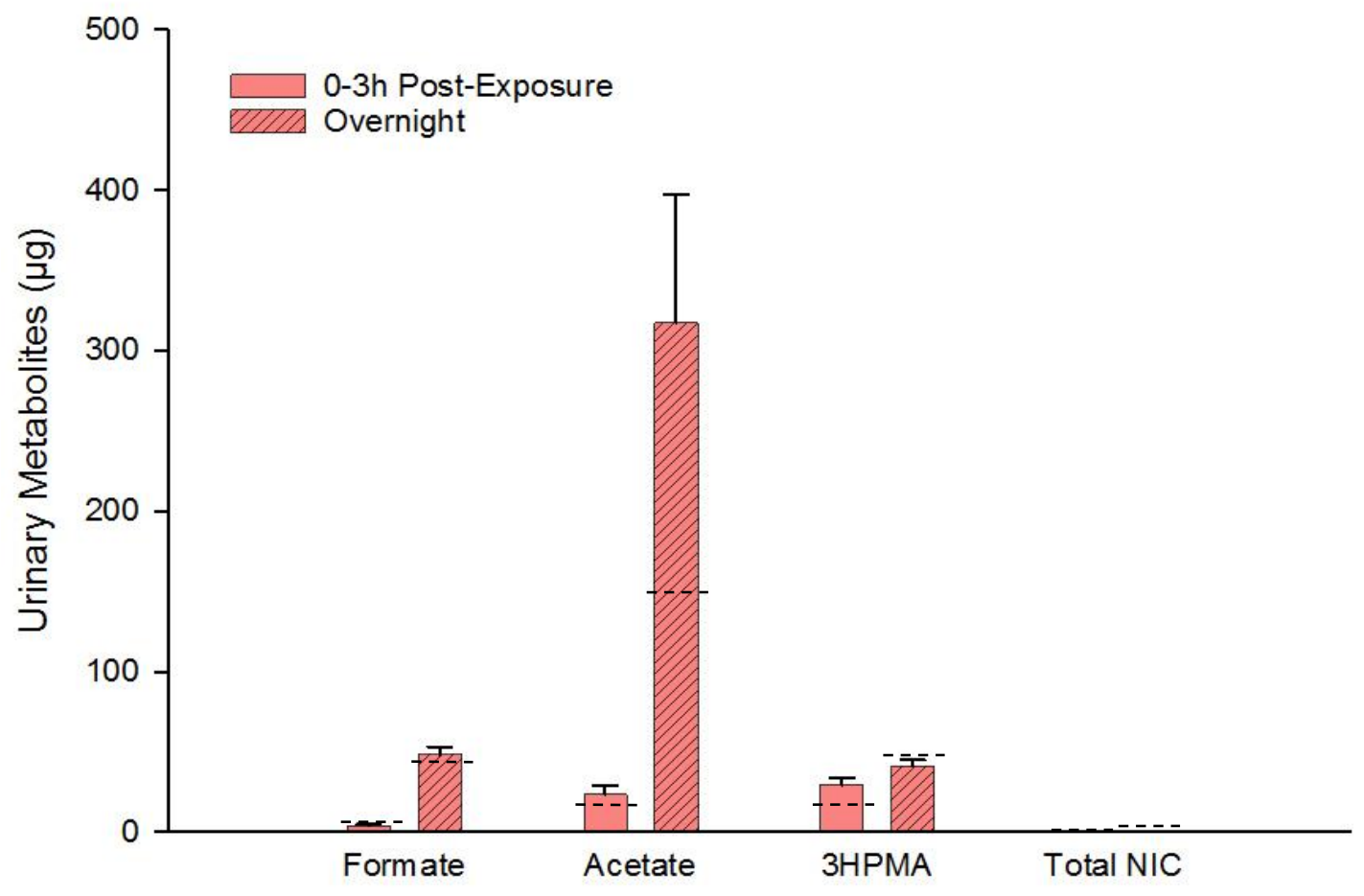

Fig. 4.9. Urinary metabolites after acute propylene glycol:vegetable glycerin (PG:VG; 30\%:70\%) exposure. Male mice were exposed (6h) to PG:VG (30\%:70\%) after which urine was collected at $0-1,1-2,2-3$, and 3-16h (overnight) and aldehyde and tobacco alkaloid metabolites quantified. Values are mean $\pm \mathrm{SE}$ ( $\mathrm{n}=4$ mice per group). Dashed lines indicate metabolite measures $(\mu \mathrm{g})$ after HEPA exposure (6h). Abbr.: 3HPMA, 3hydroxypropylmercapturic acid; HPMMA, 3-hydroxy-1-methylpropylmercapturic acid; PG:VG, propylene glycol:vegetable glycerin; Total NIC, total urinary nicotine metabolites (nicotine, cotinine, and trans-3'-hydroxycotinine). 
than that measured after air exposure (Fig. 4.8). Levels of formate and acetate after the overnight (3-16h) collections were elevated compared with levels 0-3h post-exposure with either ratio of PG:VG; these elevations are likely related to generation by food, as mice were given free access to food during this collection period (Figs. 4.8 \& 4.9).

\section{Systemic Toxicity of Acute Exposure to Inhaled PG:VG}

Because PG and VG, two humectants commonly used in e-cig devices, have been shown to generate measurable levels of aldehydes $(168,214-216)$, we tested for changes in systemic and hematological biomarkers in female mice exposed to PG:VG (30\%:70\%). PG:VG-exposed female mice showed a greater change in body weight during the exposure period ( $-2 \mathrm{~g}$ vs $-1 \mathrm{~g}$; $\mathrm{p}=0.01)$ but no changes in measured organ/body weight ratios (Table 4.7). Acute exposure had considerable effects on CBC measures. Exposed mice showed a significant decrease in leukocytes $(-47 \%$; $\mathrm{p}=0.003)$, driven by decreases in both neutrophils $(-54 \% ; \mathrm{p}=0.01)$ and lymphocytes $(-46 \% ; \mathrm{p}=0.003)$ (Table 4.8). Red blood cell levels were increased $(+6 \% ; \mathrm{p}<0.001)$ as were measures of hemoglobin $(+4 \% ; \mathrm{p}=0.002)$ and hematocrit $(+6 \%$; $\mathrm{p}=0.002)$ in PG:VG-exposed mice, while mean platelet volume (4\%; $\mathrm{p}=0.01)$ and, modestly, mean corpuscular hemoglobin $(-2 \% ; \mathrm{p}=0.06)$ were decreased

(Table 4.8). Measures of systemic biomarkers showed no changes in muscle (CK) or LDH enzymes or creatinine but did show a significant increase in cholesterol $(+15 \%$; $p=0.03)$ driven by an increase in LDL $(+26 \%$; $=0.03$ ) (Table 4.7). Conversely, ALT was significantly decreased in exposed mice $(-20 \% ; \mathrm{p}=0.02)$ (Table 4.7).

\section{Acute Exposure to Inhaled PG:VG and Circulating Angiogenic Cells}

Female mice exposed to 30\%:70\% PG:VG showed no change in the levels of CACs or Sca-1+ cells (Fig. 4.10A \& B). 
Table 4.7. Systemic parameters of female C57BL/6 mice acutely exposed to either air or propylene glycol:vegetable glycerin (PG:VG; 30\%:70\%).

\begin{tabular}{|c|c|c|}
\hline \multirow[b]{2}{*}{ Variable } & \multicolumn{2}{|c|}{ Exposure (4 days) } \\
\hline & Air Control & 30\%:70\% PG:VG \\
\hline Change in BWT (g) & $-1 \pm 0$ & $-2 \pm 0^{*}$ \\
\hline Heart/BWT (mg/g) & $5.2 \pm 0.2$ & $4.8 \pm 0.1$ \\
\hline Lung/BWT (mg/g) & $6.4 \pm 0.1$ & $6.3 \pm 0.2$ \\
\hline Liver/BWT (mg/g) & $38.8 \pm 1.3$ & $38.0 \pm 0.6$ \\
\hline Kidney/BWT (mg/g) & $12.7 \pm 0.3$ & $11.8 \pm 0.2$ \\
\hline \multicolumn{3}{|c|}{ Plasma Measurements } \\
\hline Cholesterol (mg/dL) & $44.74 \pm 1.31$ & $51.45 \pm 1.77 *$ \\
\hline HDL (mg/dL) & $25.78 \pm 1.05$ & $30.36 \pm 2.00$ \\
\hline LDL (mg/dL) & $12.80 \pm 0.85$ & $16.14 \pm 0.47 *$ \\
\hline Triglycerides (mg/dL) & $30.81 \pm 5.26$ & $26.28 \pm 1.54$ \\
\hline Albumin (g/dL) & $2.82 \pm 0.07$ & $2.98 \pm 0.07$ \\
\hline Total Protein (g/dL) & $3.78 \pm 0.11$ & $3.94 \pm 0.05$ \\
\hline ALT (U/I) & $20.20 \pm 0.56$ & $16.18 \pm 0.34^{*}$ \\
\hline AST (U/I) & $64.10 \pm 4.40$ & $57.11 \pm 4.33$ \\
\hline CK (U/I) & $164.16 \pm 11.04$ & $158.73 \pm 24.29$ \\
\hline LDH (U/I) & $125.46 \pm 8.91$ & $127.20 \pm 10.71$ \\
\hline Creatinine (mg/dL) & $0.21 \pm 0.04$ & $0.17 \pm 0.01$ \\
\hline
\end{tabular}

Values = mean \pm SE (n=5-10 mice per group); Abbr.: ALT, alanine aminotransferase; AST, aspartate aminotransferase; BWT, body weight; CK, creatine kinase; HDL, high-density lipoprotein; LDH, lactate dehydrogenase; LDL, low-density lipoprotein; PG:VG, propylene glycol:vegetable glycerin; *, p<0.05 compared with air control. 
Table 4.8. Hematological measures of female C57BL/6 mice acutely exposed to either air or propylene glycol:vegetable glycerin (PG:VG; 30\%:70\%).

\begin{tabular}{|l|c|c|}
\hline \multicolumn{1}{|c|}{ Hematological Measurements } & \multicolumn{2}{c|}{ Exposure (4 days) } \\
\hline White Blood Cell (K/uL) & Air Control & 30\%:70\% PG:VG \\
\hline Neutrophils (K/uL) & $1.34 \pm 0.16$ & $0.70 \pm 0.05^{*}$ \\
\hline Lymphocytes (K/uL) & $0.30 \pm 0.06$ & $0.14 \pm 0.01^{*}$ \\
\hline Monocytes (K/uL) & $1.00 \pm 0.11$ & $0.54 \pm 0.04^{*}$ \\
\hline Eosinophils (K/uL) & $0.04 \pm 0.01$ & $0.02 \pm 0.01$ \\
\hline Basophils (K/uL) & $0 \pm 0$ & $0 \pm 0$ \\
\hline Red Blood Cell (M/uL) & $0 \pm 0$ & $0 \pm 0$ \\
\hline Hemoglobin (g/dL) & $8.73 \pm 0.07$ & $9.29 \pm 0.07^{*}$ \\
\hline Hematocrit (\%) & $12.1 \pm 0.1$ & $12.6 \pm 0.1^{*}$ \\
\hline Mean Corpuscular Volume (fL) & $44.9 \pm 0.4$ & $47.6 \pm 0.5^{*}$ \\
\hline Mean Corpuscular Hemoglobin (pg) & $51.5 \pm 0.5$ & $51.2 \pm 0.2$ \\
\hline Mean Corpuscular Hemoglobin Concentration (g/dL) & $13.9 \pm 0.2$ & $13.6 \pm 0.1 \#$ \\
\hline Red Cell Distribution Width (\%) & $27.0 \pm 0.3$ & $26.5 \pm 0.2$ \\
\hline Platelets (K/uL) & $17.0 \pm 0.2$ & $16.8 \pm 0.1$ \\
\hline Mean Platelet Volume (fL) & $656 \pm 27$ & $710 \pm 36$ \\
\hline
\end{tabular}

Values $=$ mean \pm SE $(\mathrm{n}=10$ mice per group $) ;$ Abbr.: PG:VG, propylene glycol:vegetable glycerin; $*$, $\mathrm{p}<0.05$ compared with air control; $\#, 0.05 \leq \mathrm{p} \leq 0.10$ compared with air control. 
A.

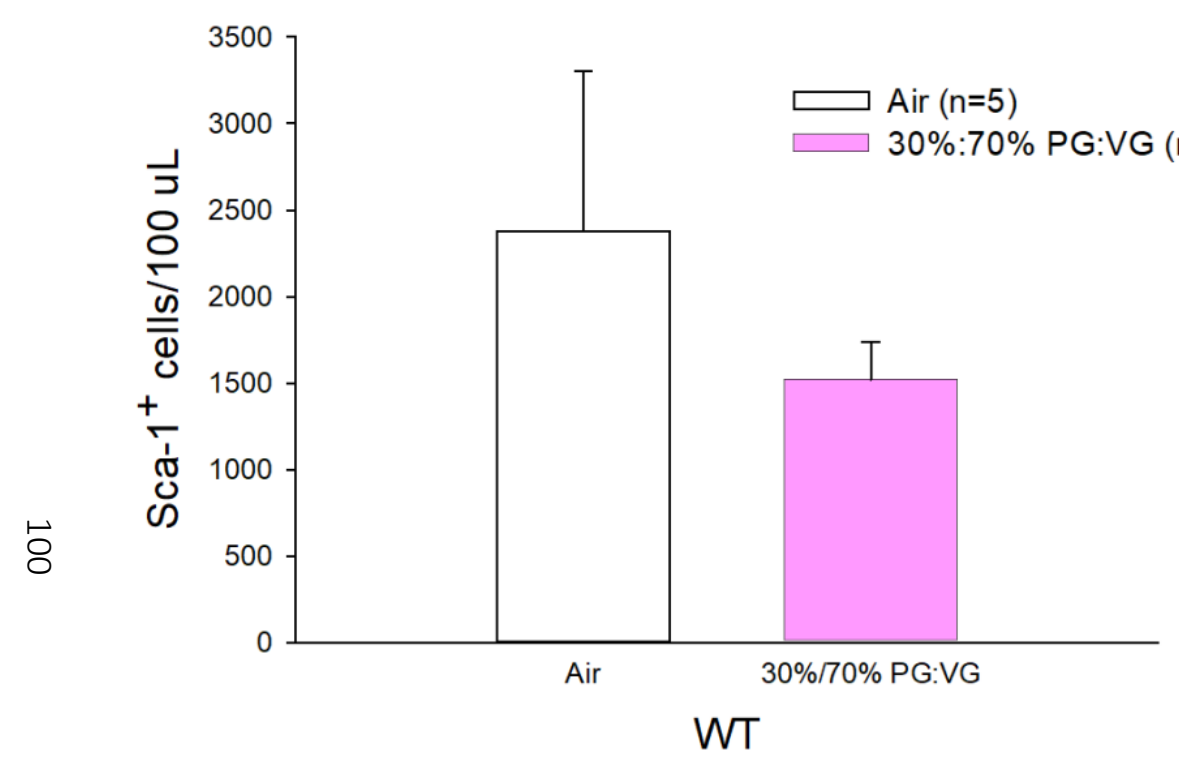

B.

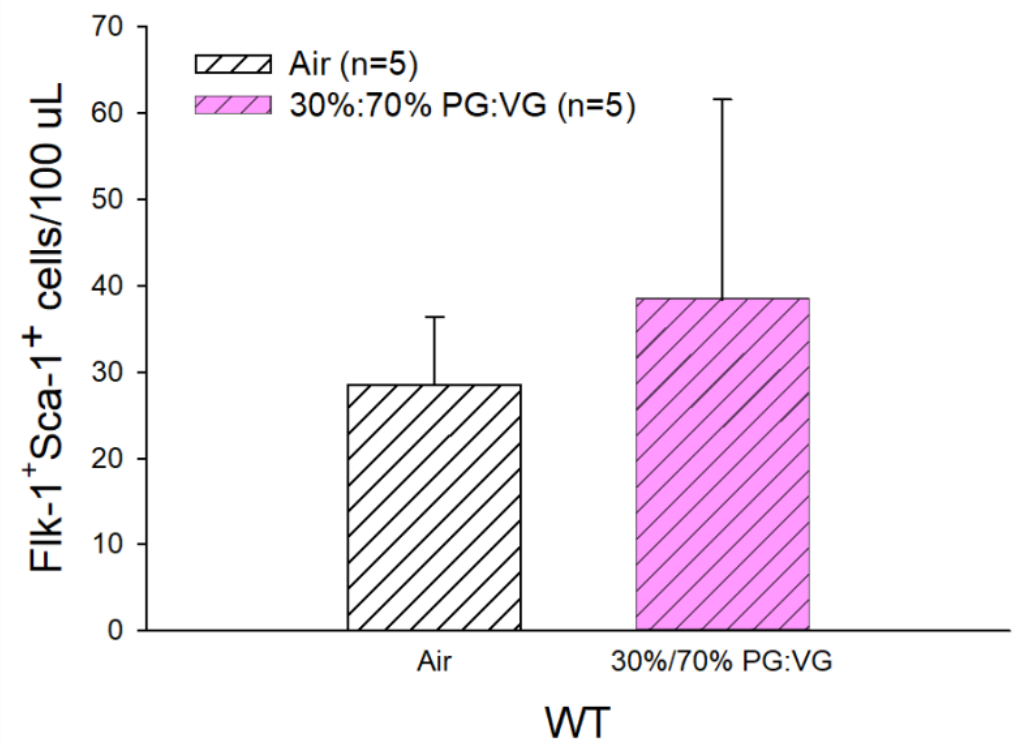

Fig. 4.10. Effects of acute propylene glycol:vegetable glycerin (PG:VG; 30\%:70\%) exposure on circulating stem cells in female mice. Hematopoietic stem cells (Sca-1+) and circulating angiogenic cells (CACs) were measured by flow cytometry in female C57BL/6J (wild type, WT) mice exposed to HEPA-filtered air or PG:VG (30\%:70\%, 6h/d) for 4 days. Levels of circulating Sca-1+ cells (A) and CACs $($ Flk-1+/Sca-1+ cells; B) were unchanged in PG:VG-exposed mice relative to air control mice. Values $=$ mean \pm SE $(n=5$ mice per group). 


\section{Discussion}

Tobacco smoking is the most significant modifiable risk factor in the development of CVD (14). As previously mentioned, tobacco smoke contains hundreds of thousands of compounds (396), and while the number of compounds generated in e-cig aerosols is still unknown, early analyses of the aerosol have shown that these devices still generate many of the harmful compounds found in $\operatorname{MCS}(96,168,400)$. The goal of this study was to examine the systemic, hematological, and endothelium-related effects of MCS, e-cig aerosol, and humectants used in e-cigs.

The results of these inhalation exposure studies revealed a number of important and novel observations and also confirmed findings previously described in the literature. Urinary analysis of male mice exposed to MCS showed that traditional cigarettes contain high levels of acrolein (measured via its urinary metabolite, 3HPMA), confirming previous data $(114,117,147)$, but levels of other measured aldehyde metabolites are low in comparison. This may indicate that acrolein is responsible for the majority of the effects noted in response to MCS exposure; the significant role of acrolein in the toxicity of MCS has already been shown $(145,149)$. Exposure to JUUL VA Tobacco and Mango showed similar levels of urinary 3HPMA, suggesting that acrolein may also play a role in the health outcomes of these flavorings. However, exposure to aerosol from a JUUL device with either VA Tobacco, Mango, or Menthol flavoring significantly increased levels of total urinary nicotine metabolites (nicotine, cotinine, and 3HC). Nicotine, an important constituent in MCS, stimulates the sympathetic nervous system and has been shown to increase heart rate, blood pressure, platelet aggregation, myocardial contractility, and atherosclerotic development (401). JUUL devices use nicotine salts to deliver nicotine to users, and these salts allow for higher levels of nicotine delivery than other e-cig devices, 
with one JUUL pod capable of providing a dose of nicotine similar to that of an entire pack of traditional cigarettes (402). Thus, it may be that the effects of JUUL devices are related to high levels of nicotine, although additional studies are needed to better understand the relationship between the levels of nicotine found in JUUL devices and our cardiovascular outcomes of interest.

Interestingly, although the levels of total urinary nicotine metabolites were increased in response to exposure to all of the flavored JUUL products compared with airexposed control mice and MCS-exposed mice, levels were significantly higher after exposure to the mango and menthol flavorings than after exposure to VA Tobacco. This may suggest that the Mango and Menthol flavorings differ from VA Tobacco in some way that allows for greater nicotine exposure. Although there has not been much study of the JUUL Mango flavoring, menthol has long been used to provide a cooling or mint flavor in traditional cigarettes. Many studies have shown that the use of mentholated cigarettes allows for greater exposure to harmful constituents of MCS compared with the use of nonmentholated cigarettes $(403,404)$, whether through the attenuation of the respiratory irritation typically induced by MCS (405) or through decreased nicotine metabolism (406). The menthol flavoring used in JUUL devices may be inducing a similar effect and thus allowing for greater nicotine exposure. These differences in measures of urinary metabolites between flavors emphasizes the need to study individual e-liquid flavors to determine how they may induce different, specific health effects. Additionally, nationally representative survey data collected from approximately 14,000 middle and high school aged individuals showed that mint and mango were the most commonly used flavorings in JUUL devices (407), indicating the importance of further study of these flavors in 
particular.

Analysis of the urinary metabolites in mice exposed to $30 \%: 70 \%$ PG:VG or 50\%:50\% PG:VG showed significant increases in 3HPMA compared with baseline measures but varied in levels of formate and acetate. Thus, as with individual flavors, these data indicate the need to determine how varying ratios of $\mathrm{PG}: \mathrm{VG}$ used in different e-cig devices may have varying health effects.

Acute exposure to MCS induced leukocyte suppression, driven by a decrease in lymphocytes. This is contrary to what is seen in epidemiological studies, where smokers show increased levels of leukocytes driven by increases in neutrophils, lymphocytes, and monocytes $(345,351-355)$. These changes are likely linked to the systemic inflammatory response activated by MCS exposure and the subsequent increase in the release of cells from the bone marrow $(408,409)$. The difference in outcomes may be due to the duration of our exposure; many epidemiological studies of smoking examine individuals who are chronic smokers and who may already have a significantly altered inflammatory response, while our mice are naïve animals never exposed to MCS or other air pollutants and whose response may be more similar to that seen in non-smokers.

In fact, analysis of more than 7,000 never-smoking adults from the third National Health and Nutrition Examination Survey found that participants with low but detectable cotinine levels - that is, individuals with secondhand smoke exposure-actually had decreased leukocyte levels compared with individuals without detectable cotinine levels (410). The authors of the study do not propose a hypothesis for why their leukocyte data are contrary to that seen in other epidemiological studies, but it does suggest that there are perhaps still nuances in the relationship between leukocytes and MCS exposure that need 
to be explored, perhaps in relation to levels of circulating Sca-1+ cells, the hematological stem cell population from which leukocytes are derived. Acute MCS exposure in the current study did not change levels of CACs but did significantly decrease levels of Sca$1+$ cells, and although the production of leukocytes occurs within the bone marrow, the decreased levels of Sca-1+ cells in the circulation may reflect decreased levels of these stem cells within the bone marrow as well, thus limiting leukocyte production. Additional studies of these stem cells in the bone marrow after MCS exposure could help clarify these outcomes.

Additional studies of the hematological response to MCS largely show that smoking increases several erythrocytic measures, including hemoglobin and platelets (345, $355,411-413)$; levels of red blood cells are also often found to be increased $(345,414)$, although this is not always observed (355). Changes in erythrocytic measures, such as increased levels of hemoglobin, that are commonly seen in smokers are believed to be a compensatory mechanism to account for lower blood oxygen levels. Smoking introduces other gases such as carbon monoxide into the bloodstream where they can displace oxygen and lead to the activation of compensatory pathways that upregulate the production of hematological factors. Changes in mean corpuscular volume, mean corpuscular hemoglobin, and mean corpuscular hemoglobin concentration are also indices based on the composition of the hemoglobin found in red blood cells, and as hemoglobin changes in response to MCS exposure, so do these indices. The current MCS study confirms these findings; data reveal that the exposed mice have altered erythrocytic measures, showing significant changes in hemoglobin, hematocrit, mean corpuscular hemoglobin, and mean corpuscular hemoglobin concentration, as well as mean platelet volume, an indicator of 
platelet size. The presence of these changes but not changes within leukocytes which are also commonly noted in smokers may indicate that changes in the hematological parameters occur earlier than those in leukocyte levels, although additional studies including chronic exposures to MCS are needed to confirm this hypothesis.

Electronic tobacco products such as e-cigs have not been in use for a period long enough to thoroughly determine the chronic outcomes of use, but acute studies using e-cig aerosol or various e-cig flavoring chemicals have shown that they have the potential to contribute to the development of CVD in a fashion similar to traditional cigarettes, including the development of endothelium dysfunction $(190,241)$, platelet activation and aggregation (370-372), and increases in markers of inflammation $(415,416)$. The results of our acute blu Plus+ e-cig exposure study largely agree with these early reports of e-cig toxicity. Leukocyte count was significantly decreased with exposure, driven by decreases in neutrophils, lymphocytes, and monocytes, and a handful of erythrocytic measures, including hemoglobin, were increased in response to exposure. Measures of endothelium function, however, did not indicate that blu Plus+ e-cig aerosol was contributing to dysfunction, as there was no change in the level of CACs in response to exposure. As there were also no change in CACs after MCS exposure, these outcomes may indicate that the endothelium dysfunction noted in response to exposure to these tobacco products takes longer than four days to develop. Nonetheless, the fact that MCS- and e-cig aerosolexposures both show a number of similar outcomes that could contribute to the development of CVD may indicate that these outcomes are being driven by shared constituents, a premise that may be particularly important to the FDA as they seek to regulate ENDS. 
Because risk for and prevalence of CVD have been shown to differ between men and women (417-422), we also examined the effects of tobacco product exposures on female mice. Like their MCS- and blu Plus+-exposed male counterparts, female mice acutely (4d) exposed to JUUL Mango showed a significant decrease in overall leukocyte count in addition to changes in erythrocytic measures, as did female mice acutely (4d) exposed to $30 \%: 70 \%$ PG:VG. However, female mice exposed to JUUL Mango also showed modest decreases in levels of both Sca-1+ cells and CACs; although MCS exposure decreased the level of Sca-1+ cells in male mice, there was no change in CACs, and neither male mice exposed to blu Plus+ e-cig aerosol or female mice exposed to 30\%:70\% PG:VG showed changes in either cell type. Measures of systemic biomarkers showed that while female mice exposed to JUUL Mango only had minimal effects (modestly increased albumin and AST), female mice exposed to 30\%:70\% PG:VG had significantly increased cholesterol, driven by an increase in LDL. The risk of coronary heart disease has been correlated with levels of LDL (423), and a high level of cholesterol is a known risk factor for ischemic and coronary heart diseases (424). Although males are more likely than females to use traditional cigarettes $(182,325,425,426)$, and early reports of e-cig use suggest that males are also more likely than females to use these devices $(182,325,427$ 429), there is preliminary data indicating that female smokers may have a greater prevalence of tobacco-related negative health outcomes than male smokers $(426,430)$. The change in Sca-1+ cells and CACs noted in female mice exposed to JUUL Mango may indicate a sex-dependent susceptibility to some component of this particular flavor, as these changes are not seen after exposure to $30 \%: 70 \%$ PG:VG, the ratio of humectants commonly used in JUUL devices, in female mice. These data may also indicate that the 
development of endothelium dysfunction is one of the earliest changes noted in response to exposure to JUUL Mango aerosol. Conversely, the change in cholesterol noted in 30\%:70\% PG:VG-exposed female mice may indicate that the e-cig humectants are first affecting systemic biomarkers rather than inducing changes in the endothelium. Additional studies are needed to determine whether these effects are seen in male mice exposed to 30\%:70\% PG:VG and JUUL Mango, to examine potential sex-dependent differences related to other e-cig flavors, and to better understand the vascular, hematological, and systemic effects of other e-cig flavors in general. Likewise, chronic exposure to both MCS and e-cig aerosols could reveal further sex-dependence in biomarkers of cardiovascular health

In summary, the studies presented in this chapter have demonstrated the impacts of traditional and emerging tobacco products on systemic and vascular health. In particular, these studies showed that exposure to e-cig aerosol (blu Plus+ and JUUL) produced levels of urinary aldehyde and tobacco alkaloid metabolites at levels similar to those found after MCS exposure, indicating that ENDS generate harmful and potentially harmful constituents much like MCS. Furthermore, data from systemic and hematological measures indicate that, like exposure to MCS, exposure to e-cig aerosol can significantly decrease levels of leukocytes, which could put users at a higher risk for infection. The decrease in CACs seen in female mice exposed to JUUL Mango but not 30\%:70\% PG:VG may indicate the potential for endothelium injury related to this specific flavor, but a comparable study in male mice exposed to JUUL Mango is needed to determine whether this specific outcome is sex-dependent. Likewise, the increase in cholesterol and LDL seen in female mice exposed to $30 \%: 70 \%$ PG:VG may indicate the potential for significant 
systemic effects known to contribute to the development of CVD in response to humectant exposure, but, again, a comparable study in male mice is needed to determine whether these outcomes are sex-dependent. As the use of emerging tobacco products continues to increase - particularly among youths — and as diseases such as EVALI continue to impact e-cig users, these studies provide novel insight into the health impacts of e-cigs and related components. Furthermore, these studies show similarities in exposure to aerosol from ecigs and smoke from traditional cigarettes, whose roles in morbidity and mortality are wellknown, and emphasize the need to study the health effects of e-cigs in both males and females in order to curtail another tobacco-related health epidemic. 


\section{CHAPTER V}

\section{SYSTEMIC, HEMATOLOGICAL, AND ENDOTHELIUM-RELATED EFFECTS OF FORMALDEHYDE AND ACETALDEHYDE IN MICE}

\section{A. Introduction}

The saturated aldehydes formaldehyde (FR) and acetaldehyde (AA) are the smallest of the aldehydes. Both of these compounds can be found in a variety of food and drinks $(99,105,106)$, and each is produced endogenously in small amounts $(93,97,98,105,106)$. However, anthropogenic sources account for the majority of human exposure. FR is used in many consumer products, including carpets, manufactured wood products, paints, and preservatives (102), and both FR and AA are formed as products of combustion from industry, vehicle exhaust, and fires $(101-103,105,106)$. Exposure to mainstream cigarette (MCS) or secondhand smoke can significantly increase the level of exposure to FR and AA. The World Health Organization estimates that smoking 20 cigarettes a day can increase exposure to FR up to $300 \%(99,114,117)$. Most AA exposure comes from food and drinks, so any inhalation can significantly increase exposure, with studies estimating that MCS can more than double AA exposure $(107,109,117,118)$. The Institute of Medicine ranks FR and AA as two of the most significant toxins in MCS, particularly in relation to cardiovascular disease (CVD) (96). The negative cardiovascular effects of FR and AA exposure have been previously documented. FR has been shown to cause 
hematotoxicity, decreasing both red $(93,120,122)$ and white $(93,120-122)$ blood cell and platelet counts $(93,120,122)$ as well as levels of myeloid progenitor cells $(120,122)$. FR has also been linked to decreases in blood pressure, both via inhalation in rats (123) and ingestion in humans (128); in vitro studies show a concentration-dependent vascular relaxation in response to FR that supports these findings (124-127). Occupational exposures show also an association between increased exposure to FR and an increased incidence of heart disease (129-131). The cardiovascular effects of AA have largely been studied in relation to aldehyde dehydrogenase (ALDH2), the enzyme responsible for AA metabolism, and specifically the ALDH2 mutation that prevents AA metabolism, thus allowing blood levels of AA to increase (132). Increased blood levels of AA have been linked to a number of CVDs $(132,134-138)$ and changes in hematological factors (139142).

Despite the significant exposure to FR and AA from MCS - as well as from electronic cigarette (e-cig) aerosol, as these devices have been shown to generate a variety of aldehydes $(96,180,207-213)$ - and despite the high percent of the world's population affected by mutated ALDH2 and who are thus more susceptible to AA exposure (133), few studies have addressed the effect of these compounds on endothelium function. Thus, the goal of these studies was to measure concentration- and duration-dependent as well as sexdependent changes in biomarkers of vascular injury, specifically circulating angiogenic cells (CACs), and systemic and hematological biomarkers in mice exposed to FR and AA.

\section{B. Experimental Procedures}

\section{Exposures}

Animals were exposed to constituents via full-body inhalation as described in 
Chapter II, Sections B.2. Upon completion of the exposure, mice were euthanized and tissues collected as described in Chapter II, Section B.5.

\section{Urine Collection}

Urine samples were collected post-exposure as described in Chapter II, Section B.3.

\section{Urine Metabolite Quantification}

Collected urine was used to quantify levels of volatile organic compounds (VOC), saturated aldehyde, and tobacco alkaloid metabolites using analytical methods as described in Chapter II, Section B.4.

\section{Complete Blood Cell Count (CBC)}

A sample of whole blood was collected after the final day of exposure for a complete blood cell count (CBC) as described in Chapter II, Section B.6.

\section{Plasma Biomarkers Quantification}

Plasma was prepared from each sample for an analysis of a number of biomarkers as described in Chapter II, Section B.7.

\section{Circulating Angiogenic Cell (CAC) Quantification}

Flow cytometry was used to analyze the number of CACs in each sample as described in Chapter II, Section B.8.

\section{Statistical Analyses}

Statistical analyses were performed as described in Chapter II, Section B.10.

\section{Results}

1. Urinary Formaldehyde Metabolite in MCS-, PG:VG-, and FormaldehydeExposed Mice

Although exposure to FR occurs frequently through ingestion of various food 
products or by exposure to exhaust and other combustion products (100-104), the use of tobacco products results in a much greater level of exposure to FR $(93,95,99)$. Furthermore, although formate, the primary urinary metabolite of FR, has not been validated as a urinary biomarker of tobacco exposure $(431,432)$, studies have shown that it can be used in conjunction with other biomarkers as an indicator of tobacco product use or exposure $(168,293,433)$. To better understand how FR-alone exposure related to MCS exposure from a known number of cigarettes and to e-cig exposure from known ratios of PG:VG, we measured urinary formate by UPLC-MS/MS to confirm that the concentration of FR used in our FR-alone exposure was comparable to exposures from real-world sources. For this, male mice were exposed to FR ( 5 ppm, $6 \mathrm{~h}$ ), MCS (50\% of smoke of 12 KY Reference 3R4F cigarettes, $6 \mathrm{~h})$, or PG:VG (50\%:50\% or 30\%:70\%, 6h), and urine was collected immediately after the exposure sessions.

Urinary formate levels in the first three hours post-exposure were not significantly different between mice exposed to FR (5 ppm) or to 30\%:70\% PG:VG compared with baseline, but mice exposed to MCS or 50\%:50\% PG:VG showed significantly increased levels ( $\mathrm{p}=0.009$ and $\mathrm{p}=0.003$, respectively) (Fig. 5.1). Additionally, levels of formate in MCS-exposed mice were significantly higher than levels in FR-exposed mice (Fig. 5.1). Formate levels in FR-exposed mice were not significantly different from those in PG:VGexposed mice, likely indicating that the concentration of FR generated for the FR-alone exposure (5 ppm) is similar to the levels generated in PG:VG, regardless of the ratio (Fig. 5.1). Levels of formate after the overnight (3-16h) collections were elevated compared with levels $0-3 \mathrm{~h}$ post-exposure with all exposures; these elevations are likely related to generation by food, as mice were given free access to food during this collection period 


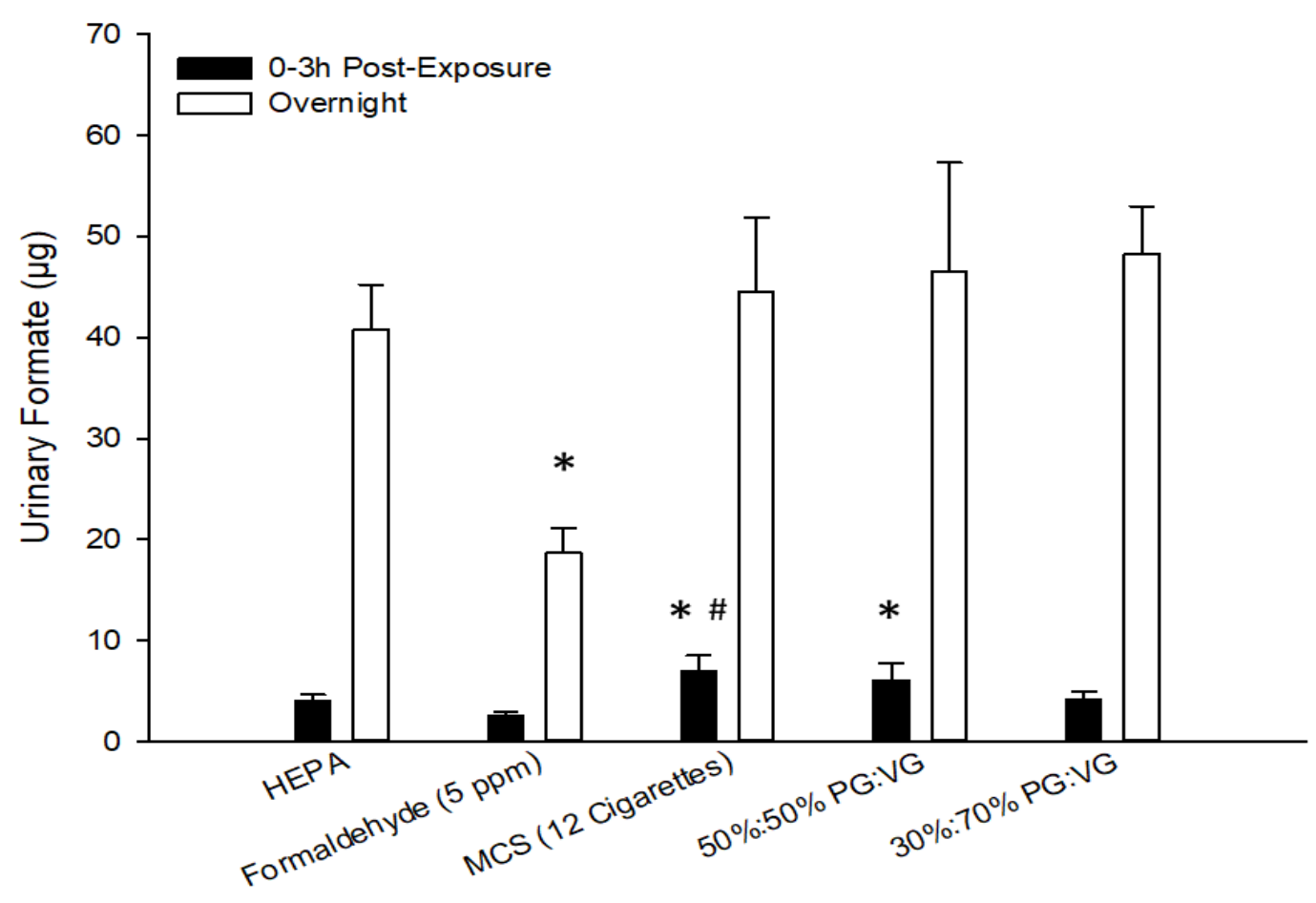

Fig. 5.1. Urinary formate levels in formaldehyde- or tobacco product-exposed male mice. Male mice were exposed (6h) to formaldehyde (5 ppm), mainstream cigarette smoke (MCS; 50\% smoke of 12 cigarettes), or propylene glycol:vegetable glycerin (PG:VG; 50\%:50\% or 30\%:70\%, 6h) after which urine was collected at $0-1,1-2,2-3$, and $3-16 \mathrm{~h}$ (overnight) and formate quantified. Values are mean $\pm \mathrm{SE}$ ( $\mathrm{n}=4-12$ mice per group). *, p $<0.05$ compared with HEPA; \#, p $<0.05$ compared with formaldehyde. 
(Fig. 5.1). Additionally, mice exposed to FR had lower levels of formate after the overnight exposure compared with baseline (Fig. 5.1).

\section{Systemic Toxicity of Two-Week Exposure to Inhaled Formaldehyde}

Because of the potential for MCS to significantly increase levels of FR exposure $(99,114,117)$, we tested for changes in systemic and hematological biomarkers in mice exposed to FR (5 ppm). Body weight and organ/body weight ratios showed significant changes between the air- and FR-exposed groups; exposure to 5 ppm FR caused decreases in final body weight $(-8 \% ; \mathrm{p}=0.02)$ and the liver/body weight ratio $(-5 \% ; \mathrm{p}=0.08)$ and an increase in the lung/body weight ratio $(+12 \%, \mathrm{p}=0.002)$ (Table 5.1). Two weeks of exposure had modest to considerable effects on $\mathrm{CBC}$ and plasma biomarkers. Exposed mice had a decreased level of leukocytes $(-46 \% ; \mathrm{p}=0.004)$, driven by decreases in all leukocyte subpopulations, but an increased level of red blood cells (+9\%; p=0.01) (Table 5.2). Likewise, hemoglobin levels $(+7 \% ; \mathrm{p}=0.02)$ were increased, while mean corpuscular hemoglobin concentration $(-2 \% ; \mathrm{p}=0.08)$, platelet count $(-17 \% ; \mathrm{p}=0.01)$, and mean platelet

volume $(-12 \% ; \mathrm{p}=0.002)$ were all decreased (Table 5.2). A low volume of plasma limited additional measurements of systemic toxicity, but exposed mice showed a significant decrease in cholesterol compared with air-exposed control mice $(-32 \% ; \mathrm{p}=0.03)$ (Table 5.1).

\section{Two-Week Exposure to Formaldehyde and Circulating Angiogenic Cells}

Decreased levels of CACs have been associated with increased CVD risk, and it is thought that CAC levels serve as a predictor of overall cardiovascular health and future cardiovascular events (66). Altered CAC levels are noted in individuals exposed to secondhand smoke (72) and during smoking cessation (318). Like the changes seen in 
Table 5.1. Systemic parameters of male C57BL/6 mice acutely exposed to either air or formaldehyde (FR; 1, 2, or 5 ppm).

\begin{tabular}{|c|c|c|c|c|c|}
\hline \multicolumn{6}{|c|}{ Exposure } \\
\hline & & \multicolumn{3}{|c|}{4 days } & 2 weeks \\
\hline Variable & Air Control & FR (1 ppm) & FR (2 ppm) & FR (5 ppm) & FR (5 ppm) \\
\hline Final BWT (g) & $28 \pm 0$ & $29 \pm 0$ & $29 \pm 1$ & $27 \pm 1$ & $26 \pm 0 *$ \\
\hline Heart/BWT (mg/g) & $5.0 \pm 0.1$ & $5.0 \pm 0.3$ & $4.9 \pm 0.1$ & $5.1 \pm 0.2$ & $4.8 \pm 0.1$ \\
\hline Lung/BWT (mg/g) & $5.6 \pm 0.1$ & $5.1 \pm 0.2$ & $5.2 \pm 0.1$ & $5.5 \pm 0.2$ & $6.2 \pm 0.1^{*}$ \\
\hline Liver/BWT (mg/g) & $42.1 \pm 0.6$ & $42.9 \pm 1.0$ & $42.3 \pm 0.8$ & $40.5 \pm 1.2$ & $40.2 \pm 1.0$ \\
\hline Kidney/BWT (mg/g) & $12.9 \pm 0.2$ & $12.6 \pm 0.2$ & $12.2 \pm 0.2^{*}$ & $11.2 \pm 0.8^{*}$ & $13.1 \pm 0.3$ \\
\hline \multicolumn{6}{|c|}{ Plasma Measurements } \\
\hline Cholesterol (mg/dL) & $96.03 \pm 3.67$ & $117.89 \pm 2.64^{*}$ & $103.83 \pm 3.13$ & $94.23 \pm 2.32$ & $65.45 \pm 5.29 *$ \\
\hline HDL (mg/dL) & $66.31 \pm 4.33$ & N/A & N/A & $67.88 \pm 2.90$ & N/A \\
\hline Triglycerides (mg/dL) & $47.84 \pm 2.20$ & $44.57 \pm 2.22$ & $33.75 \pm 3.86^{*}$ & $43.64 \pm 2.01$ & $50.48 \pm 2.15$ \\
\hline Albumin (g/dL) & $3.37 \pm 0.06$ & $3.71 \pm 0.05^{*}$ & $3.66 \pm 0.05^{*}$ & $3.21 \pm 0.06$ & N/A \\
\hline Total Protein (g/dL) & $4.74 \pm 0.08$ & $4.50 \pm 0.07 \#$ & $4.67 \pm 0.05$ & $5.18 \pm 0.13^{*}$ & N/A \\
\hline ALT (U/I) & $24.40 \pm 1.65$ & $19.99 \pm 0.71$ & $24.84 \pm 1.82$ & $38.44 \pm 7.75 \#$ & N/A \\
\hline $\operatorname{AST}(\mathrm{U} / \mathrm{I})$ & $77.33 \pm 7.55$ & $40.63 \pm 2.72 *$ & $60.61 \pm 3.96$ & $104.26 \pm 14.34 *$ & N/A \\
\hline CK (U/I) & $277.82 \pm 26.67$ & $169.65 \pm 12.31 *$ & $267.89 \pm 34.08$ & $314.13 \pm 68.39$ & N/A \\
\hline LDH (U/I) & $230.89 \pm 21.17$ & $156.74 \pm 7.25^{*}$ & $263.01 \pm 24.92$ & $188.06 \pm 23.20$ & N/A \\
\hline Creatinine (mg/dL) & $0.31 \pm 0.01$ & $0.30 \pm 0.01$ & $0.38 \pm 0.01 *$ & $0.37 \pm 0.01^{*}$ & N/A \\
\hline
\end{tabular}

Values = mean \pm SE (n=6-11 mice per group); Abbr.: ALT, alanine aminotransferase; AST, aspartate aminotransferase; BWT, body weight; CK, creatine kinase; FR, formaldehyde; HDL, high-density lipoprotein; LDH, lactate dehydrogenase; *, p<0.05 compared with air control; \#, $0.05 \leq \mathrm{p} \leq 0.10$ compared with air control. 
Table 5.2. Hematological measures of male C57BL/6 mice acutely exposed to either air or formaldehyde (FR; 1,2 , or 5 ppm).

\begin{tabular}{|l|l|c|c|c|c|}
\hline & & \multicolumn{3}{|c|}{ 4 days weeks } \\
\hline \multicolumn{1}{|c|}{ Hematological Measurements } & Air Control & FR (1 ppm) & FR (2 ppm) & FR (5 ppm) & FR (5 ppm) \\
\hline White Blood Cell (K/uL) & $2.35 \pm 0.18$ & $2.11 \pm 0.23$ & $2.06 \pm 0.10$ & $2.94 \pm 0.49$ & $1.27 \pm 0.15^{*}$ \\
\hline Neutrophils (K/uL) & $0.66 \pm 0.11$ & $0.27 \pm 0.03^{*}$ & $0.46 \pm 0.04$ & $0.79 \pm 0.16$ & $0.31 \pm 0.05 \#$ \\
\hline Lymphocytes (K/uL) & $1.60 \pm 0.12$ & $1.71 \pm 0.19$ & $1.44 \pm 0.11$ & $2.07 \pm 0.40$ & $0.92 \pm 0.11^{*}$ \\
\hline Monocytes (K/uL) & $0.07 \pm 0.01$ & $0.08 \pm 0.02$ & $0.06 \pm 0.01$ & $0.06 \pm 0.01$ & $0.04 \pm 0.01^{*}$ \\
\hline Eosinophils (K/uL) & $0.01 \pm 0$ & $0.06 \pm 0.01^{*}$ & $0.01 \pm 0$ & $0.01 \pm 0.01$ & $0 \pm 0 \#$ \\
\hline Basophils (K/uL) & $0.01 \pm 0$ & $0 \pm 0$ & $0 \pm 0$ & $0.01 \pm 0$ & $0 \pm 0 *$ \\
\hline Red Blood Cell (M/uL) & $9.54 \pm 0.16$ & $9.49 \pm 0.13$ & $9.13 \pm 0.26$ & $8.45 \pm 0.17^{*}$ & $10.39 \pm 0.11^{*}$ \\
\hline Hemoglobin (g/dL) & $13.6 \pm 0.2$ & $13.6 \pm 0.1$ & $12.3 \pm 0.3^{*}$ & $12.3 \pm 0.2 \#$ & $14.6 \pm 0.1^{*}$ \\
\hline Hematocrit (\%) & $41.3 \pm 0.7$ & $45.1 \pm 0.5^{*}$ & $39.0 \pm 1.1 \#$ & $35.0 \pm 0.7^{*}$ & $43.9 \pm 0.5$ \\
\hline Mean Corpuscular Volume (fL) & $43.3 \pm 0.3$ & $47.5 \pm 0.3^{*}$ & $42.7 \pm 0.2$ & $41.5 \pm 0.3^{*}$ & $42.2 \pm 0.4$ \\
\hline Mean Corpuscular Hemoglobin & $14.2 \pm 0.1$ & $14.3 \pm 0.1$ & $13.6 \pm 0.2^{*}$ & $14.6 \pm 0.1 \#$ & $14.0 \pm 0.1 \#$ \\
\hline $\begin{array}{l}\text { Mean Corpuscular Hemoglobin } \\
\text { Concentration (g/dL) }\end{array}$ & $32.9 \pm 0.3$ & $30.1 \pm 0.2^{*}$ & $31.7 \pm 0.3$ & $35.3 \pm 0.4^{*}$ & $33.2 \pm 0.3$ \\
\hline Red Cell Distribution Width (\%) & $18.4 \pm 0.2$ & N/A & $17.6 \pm 0.2^{*}$ & $17.6 \pm 0.4$ & $17.9 \pm 0.3$ \\
\hline Platelets (K/uL) & $735 \pm 24$ & $800 \pm 16$ & $810 \pm 34$ & $715 \pm 30$ & $612 \pm 55^{*}$ \\
\hline Mean Platelet Volume (fL) & $4.8 \pm 0.1$ & $6.0 \pm 0 *$ & $4.7 \pm 0.1$ & $4.5 \pm 0$ & $4.3 \pm 0.1^{*}$ \\
\hline
\end{tabular}

Values $=$ mean \pm SE ( $=6$-11 mice per group); Abbr.: FR, formaldehyde; ${ }^{*}, \mathrm{p}<0.05$ compared with air control; \#, $0.05 \leq \mathrm{p} \leq 0.10$ compared with air control. 
response to tobacco smoke, there was a significant decrease in the level of CACs (Flk$1+/$ Sca- $1+$ cells $)$ after two weeks of FR exposure (5 ppm; $-56 \%, \mathrm{p}=0.003)$ (Fig. 5.2B). Levels of Sca-1+ cells, the larger hematopoietic stem cell population from which CACs are derived, were also significantly decreased compared with air-exposed control mice (-50\%; $\mathrm{p}=0.01)($ Fig. 5.2A).

\section{Systemic Toxicity of Acute Exposure to Inhaled Formaldehyde}

Because two weeks of exposure to FR (5 ppm) had specific systemic toxicity that included changes in body weight, CBC (leukocytes, red blood cells, hemoglobin), plasma factors (cholesterol), and CACs (decreased), we performed acute FR exposures to better understand the temporal relationships between these factors. Thus, we tested whether acute FR exposures would induce rapid toxicity that was otherwise compensated for in a longer setting. Acute exposure of male mice (1, 2, or $5 \mathrm{ppm} ; 4$ days) had limited effects on organ and body weights (or ratios) or in leukocytes, but caused significant changes in erythrocytes and related measures (Tables $5.1 \& 5.2$ ). Exposure to a higher concentration of FR ( $5 \mathrm{ppm})$ caused no change in overall body weight but induced a significant decrease in kidney/body weight ratio $(-13 \% ; \mathrm{p}=0.001)$ (Table 5.1). Although the overall leukocyte count and counts of subpopulations were unchanged, FR-exposed mice showed decreased red blood cell count $(-11 \% ; \mathrm{p}=0.003)$, hemoglobin $(-9 \% ; \mathrm{p}=0.10)$, hematocrit $(-15 \%$; $\mathrm{p}<0.001)$, and mean corpuscular volume $(-4 \% ; \mathrm{p}=0.003)$ but increased mean corpuscular hemoglobin $(+3 \% ; \mathrm{p}=0.06)$ and mean corpuscular hemoglobin concentration $(+7 \%$; $\mathrm{p}=0.002$ ) (Table 5.2). Acute exposure to FR (5 ppm) did not affect plasma levels of CK (striated muscle) or LDH (non-specific cell toxicity) enzymes, but did increase levels of plasma total protein $(+9 \% ; \mathrm{p}=0.01)$, creatinine $(+20 \%$; $=0.03)$, AST (liver) $(+35 \%$; 
A.

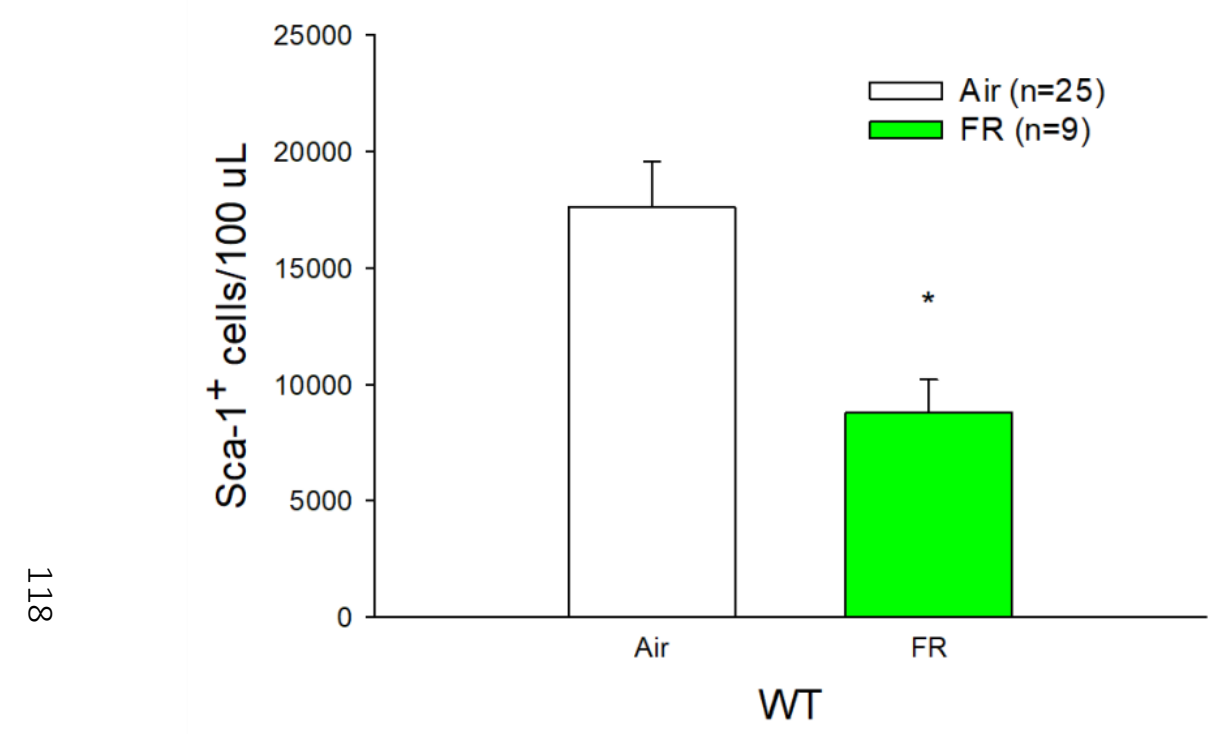

B.

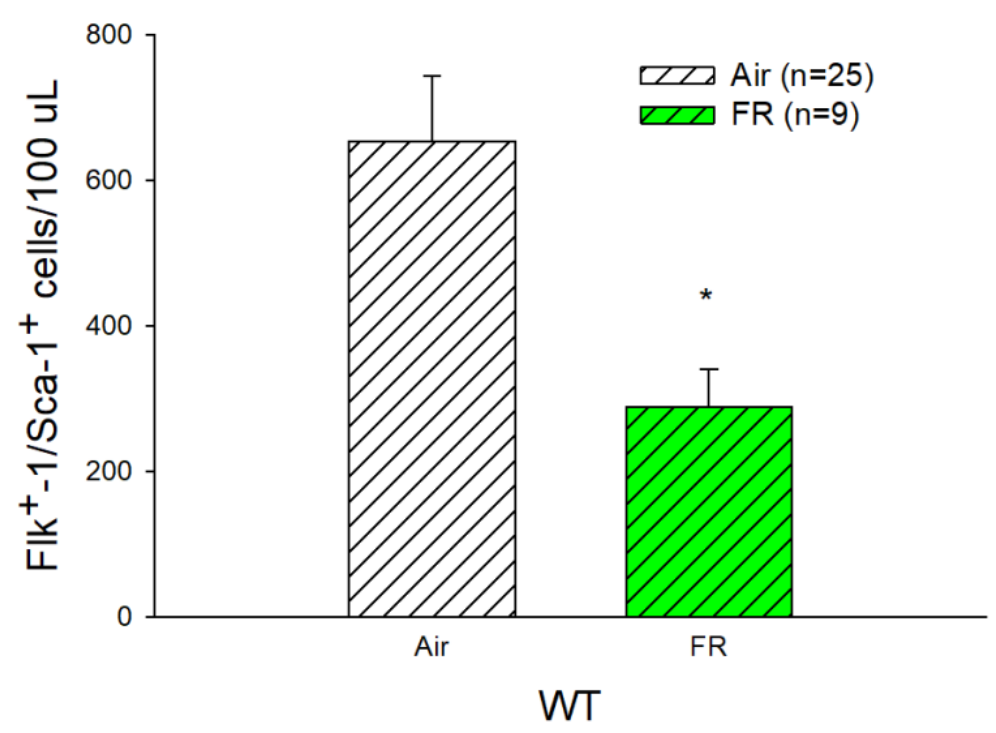

Fig. 5.2. Effects of two-week formaldehyde (FR; 5 ppm) exposure on circulating stem cells in male mice. Hematopoietic stem cells (Sca-1+) and circulating angiogenic cells (CACs) were measured by flow cytometry in male C57BL/6J (wild type, WT) mice exposed to HEPA-filtered air or formaldehyde $(5 \mathrm{ppm}, 6 \mathrm{~h} / \mathrm{d})$ for two weeks. After two weeks of exposure to FR, the levels of both circulating Sca-1+ cells $(\mathbf{A})$ and CACs $($ Flk-1+/Sca-1+ cells $)(\mathbf{B})$ were significantly decreased in WT mice. Values $=$ mean \pm SE $(n=9$ 25 mice per group). *, $\mathrm{p}<0.05$ compared with air control. 
$\mathrm{p}=0.03)$, and, modestly, ALT (liver) $(+58 \%$; $\mathrm{p}=0.07)$ compared with the air-exposed control group (Table 5.1).

Unsurprisingly, acute (4d) exposure to FR at lower concentrations (1 and 2 ppm) showed similar but diminished effects overall on markers of systemic and hematological toxicity than $5 \mathrm{ppm}$ exposure. Exposure to FR (2 ppm) showed a similar decrease in kidney/body weight ratio $(-6 \% ; \mathrm{p}=0.04)$ but no changes in leukocytes (Tables 5.1 \& 5.2). Measures of hemoglobin and hematocrit were likewise decreased $(-9 \% ; \mathrm{p}=0.02$ and $-6 \%$; $\mathrm{p}=0.08$, respectively), but red cell distribution width was also increased $(-4 \% ; \mathrm{p}=0.02)$, while mean corpuscular hemoglobin was decreased rather than increased as was seen at a higher FR concentration $(5 \mathrm{ppm})(-5 \%$; $<$ <0.001) (Table 5.2). Creatinine was again increased $(+23 \%$; $<<0.001)$, and changes in triglycerides $(-29 \% ; \mathrm{p}=0.003)$ and albumin $(+9 \% ; \mathrm{p}=0.002)$ were also induced at this level of exposure $(2 \mathrm{ppm})$ (Table 5.1). At the lowest-tested concentration of FR (1 ppm), acute (4d) exposure caused no changes in organ or body weights (or ratios) (Table 5.1). Plasma levels of triglycerides, ALT, and creatinine remained unchanged, however, levels of plasma cholesterol and albumin were increased $(+23 \% ; \mathrm{p}=0.004$ and $+10 \% ; \mathrm{p}=0.01$, respectively $)$, while levels of plasma total protein (5\%; $\mathrm{p}=0.08)$, AST $(-47 \% ; \mathrm{p}=0.001), \mathrm{CK}(-39 \% ; \mathrm{p}=0.04)$, and LDH $(-32 \% ; \mathrm{p}=0.01)$ were decreased (Table 5.1). Although there was no change in overall leukocyte count, neutrophil count was decreased $(-60 \% ; \mathrm{p}=0.04)$ and eosinophils increased $(+438 \%$; $\mathrm{p}<0.001)$ (Table 5.2). Contrary to changes seen at higher FR concentrations ( 2 and/or 5 ppm), both hematocrit $(+9 \% ; \mathrm{p}=0.01)$ and mean corpuscular volume $(+10 \% ; \mathrm{p}<0.001)$ were increased and mean corpuscular hemoglobin concentration decreased $(-8 \% ; \mathrm{p}<0.001)$ compared with air-exposed control mice; mean platelet volume was also increased with 
low level (1 ppm) FR exposure (+24\%; p=0.002) (Table 5.2).

In female mice, acute exposure to FR ( 2 or 5 ppm; 4 days) had limited to modest effects on organ and body weights (or ratios), CBC, or plasma biomarkers (Tables 5.3 \& 5.4). For example, female mice acutely exposed to 2 ppm FR had no change in overall body weight or organ/body weight ratios (Table 5.3) and only increases in eosinophils $(+231 \% ; \mathrm{p}=0.01)$ and basophils $(+141 \% ; \mathrm{p}=0.09)$ (Table 5.4). Plasma levels in liver (ALT, AST) and CK (muscle) enzymes and creatinine were unchanged, but LDH and, modestly, albumin were increased $(+37 \% ; \mathrm{p}=0.03$ and $+6 \%$; $=0.09$, respectively) (Table 5.3). Surprisingly, 4 days of FR (5 ppm) exposure in female mice has a similarly minimal effect on markers of systemic toxicity. Cholesterol $(-32 \% ; \mathrm{p}=0.02)$, triglycerides $(-14 \% ; \mathrm{p}=0.05)$, albumin $(-14 \% ; \mathrm{p}=0.01)$, and total protein $(-9 \% ; \mathrm{p}=0.03)$ were all decreased, as were monocyte levels $(-37 \% ; \mathrm{p}=0.04)$ and mean platelet volume $(-9 \% ; \mathrm{p}=0.004)$ (Table 5.3).

\section{Acute Exposure to Formaldehyde and Circulating Angiogenic Cells}

Acute (4d) exposure to 5 ppm FR increased the levels of CACs in male mice $(+62 \% ; \mathrm{p}=0.002)$, but there was no change in the level of Sca-1+ cells (Fig. 5.3A \& B). Acute (4d) exposure to 2 ppm FR did not change the level of Sca-1+ cells or CACs (Fig. 5.4A \& B). Likewise, female mice had no change in the levels of CACs or Sca-1+ cells with acute FR (2 or 5 ppm) exposure (Figs. 5.5A \& B and 5.6A \& B).

\section{Urinary Acetaldehyde Metabolite in MCS-, PG:VG-, and Acetaldehyde- Exposed Mice}

Although exposure to AA occurs largely through the ingestion of various food products, particularly via alcohol consumption (105-107), the use of tobacco products can result in a much greater level of exposure to AA $(93,95,99)$. Like formate, acetate, the 
Table 5.3. Systemic parameters of female C57BL/6 mice acutely exposed to either air or formaldehyde (FR; 2 or 5 ppm).

\begin{tabular}{|c|c|c|c|}
\hline \multicolumn{4}{|c|}{ Exposure } \\
\hline Variable & Air Control & FR (2 ppm) & FR (5 ppm) \\
\hline Final BWT (g) & $26 \pm 1$ & $25 \pm 1$ & $26 \pm 1$ \\
\hline Heart/BWT (mg/g) & $4.8 \pm 0.1$ & $5.0 \pm 0.1$ & N/A \\
\hline Lung/BWT (mg/g) & $7.0 \pm 0.3$ & $6.6 \pm 0.2$ & N/A \\
\hline Liver/BWT (mg/g) & $43.5 \pm 1.0$ & $41.9 \pm 1.7$ & N/A \\
\hline Kidney/BWT (mg/g) & $11.5 \pm 0.2$ & $11.8 \pm 0.2$ & N/A \\
\hline \multicolumn{4}{|c|}{ Plasma Measurements } \\
\hline Cholesterol (mg/dL) & $69.58 \pm 5.64$ & $78.89 \pm 3.13$ & $48.09 \pm 4.01 *$ \\
\hline HDL (mg/dL) & $62.82 \pm 2.73$ & $55.89 \pm 3.15$ & N/A \\
\hline Triglycerides (mg/dL) & $31.17 \pm 2.04$ & $35.76 \pm 3.51$ & $26.83 \pm 1.82 \#$ \\
\hline Albumin (g/dL) & $3.03 \pm 0.08$ & $3.21 \pm 0.06 \#$ & $2.60 \pm 0.06^{*}$ \\
\hline Total Protein (g/dL) & $4.93 \pm 0.23$ & $5.17 \pm 0.10$ & $4.47 \pm 0.20 *$ \\
\hline ALT (U/I) & $20.32 \pm 1.31$ & $21.24 \pm 1.43$ & $16.77 \pm 1.29$ \\
\hline AST (U/I) & $77.51 \pm 5.80$ & $94.87 \pm 13.23$ & $77.62 \pm 15.65$ \\
\hline CK (U/I) & $240.68 \pm 33.97$ & $318.58 \pm 46.05$ & $229.07 \pm 50.25$ \\
\hline LDH (U/I) & $158.19 \pm 13.62$ & $217.01 \pm 28.28 *$ & $139.31 \pm 15.86$ \\
\hline Creatinine (mg/dL) & $0.29 \pm 0.02$ & $0.32 \pm 0.01$ & $0.25 \pm 0.01$ \\
\hline
\end{tabular}

Values = mean \pm SE (n=6-10 mice per group); Abbr.: ALT, alanine aminotransferase; AST, aspartate aminotransferase; BWT, body weight; CK, creatine kinase; FR, formaldehyde; HDL, high-density lipoprotein; LDH, lactate dehydrogenase; *, p<0.05 compared with air control; \#, $0.05 \leq \mathrm{p} \leq 0.10$ compared with air control. 
Table 5.4. Hematological measures of female C57BL/6 mice acutely exposed to either air or formaldehyde (FR; 2 or 5 ppm).

\begin{tabular}{|l|c|c|c|}
\hline \multicolumn{3}{|c|}{ Exposure } \\
\hline \multicolumn{1}{|c|}{ Hematological Measurements } & Air Control & FR (2 ppm) & FR (5 ppm) \\
\hline White Blood Cell (K/uL) & $2.20 \pm 0.26$ & $2.24 \pm 0.24$ & $2.13 \pm 0.34$ \\
\hline Neutrophils (K/uL) & $0.55 \pm 0.07$ & $0.57 \pm 0.07$ & $0.75 \pm 0.20$ \\
\hline Lymphocytes (K/uL) & $1.57 \pm 0.21$ & $1.54 \pm 0.18$ & $1.34 \pm 0.19$ \\
\hline Monocytes (K/uL) & $0.08 \pm 0.01$ & $0.09 \pm 0.01$ & $0.05 \pm 0.01^{*}$ \\
\hline Eosinophils (K/uL) & $0.01 \pm 0.01$ & $0.03 \pm 0.01 *$ & $0 \pm 0$ \\
\hline Basophils (K/uL) & $0 \pm 0$ & $0.01 \pm 0.01 \#$ & $0 \pm 0$ \\
\hline Red Blood Cell (M/uL) & $8.46 \pm 0.23$ & $8.68 \pm 0.27$ & $8.93 \pm 0.20$ \\
\hline Hemoglobin (g/dL) & $12.2 \pm 0.4$ & $13.0 \pm 0.3$ & $12.1 \pm 0.2$ \\
\hline Hematocrit (\%) & $35.9 \pm 1.0$ & $37.6 \pm 1.1$ & $37.5 \pm 0.7$ \\
\hline Mean Corpuscular Volume (fL) & $42.5 \pm 0.3$ & $43.3 \pm 0.4$ & $42.0 \pm 0.4$ \\
\hline Mean Corpuscular Hemoglobin & $14.5 \pm 0.3$ & $15.0 \pm 0.2$ & $13.6 \pm 0.2$ \\
\hline $\begin{array}{l}\text { Mean Corpuscular Hemoglobin } \\
\text { Concentration (g/dL) }\end{array}$ & $34.0 \pm 14.0$ & $34.5 \pm 0.3$ & $32.3 \pm 0.2$ \\
\hline Red Cell Distribution Width (\%) & $17.5 \pm 0.2$ & $17.5 \pm 0.2$ & $17.6 \pm 0.2$ \\
\hline Platelets (K/uL) & $803 \pm 45$ & $737 \pm 31$ & $684 \pm 49$ \\
\hline Mean Platelet Volume (fL) & $4.9 \pm 0.1$ & $4.9 \pm 0.1$ & $4.4 \pm 0.1 *$ \\
\hline
\end{tabular}

Values $=$ mean $\pm \mathrm{SE}$ ( $\mathrm{n}=6$-10 mice per group); Abbr.: FR, formaldehyde; $*$, $\mathrm{p}<0.05$ compared with air control; \#, $0.05 \leq \mathrm{p} \leq 0.10$ compared with air control. 
A

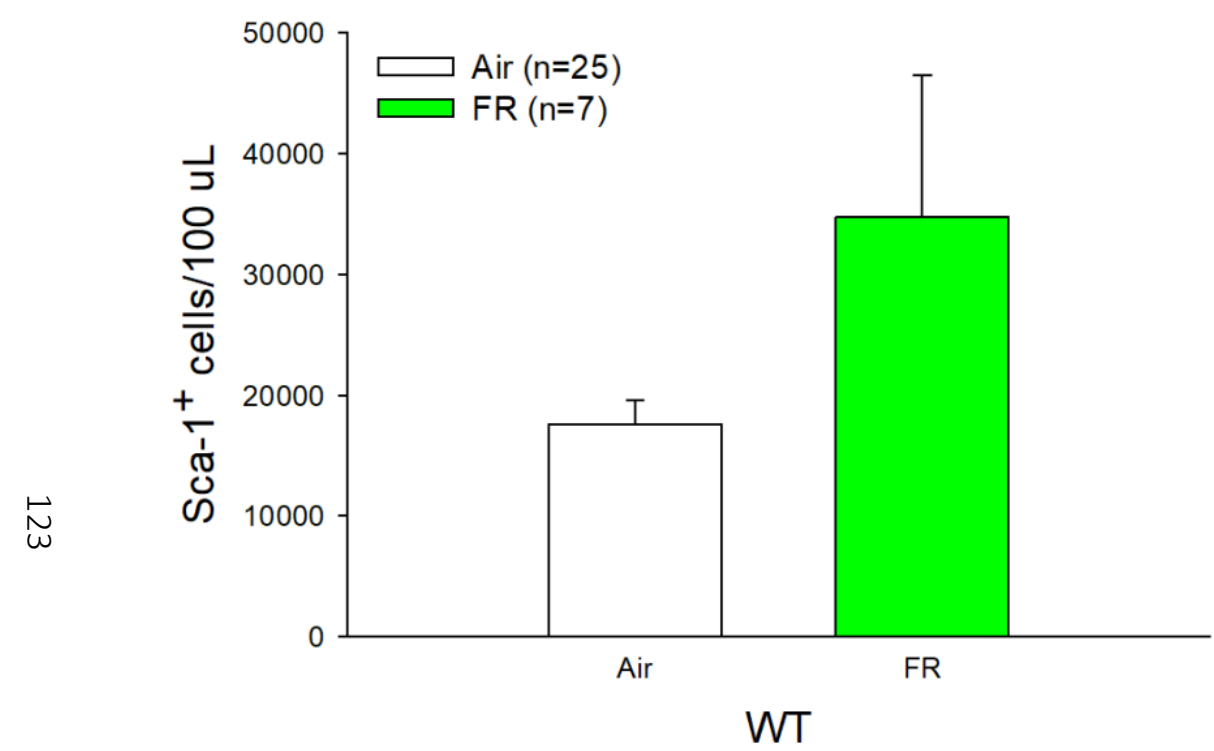

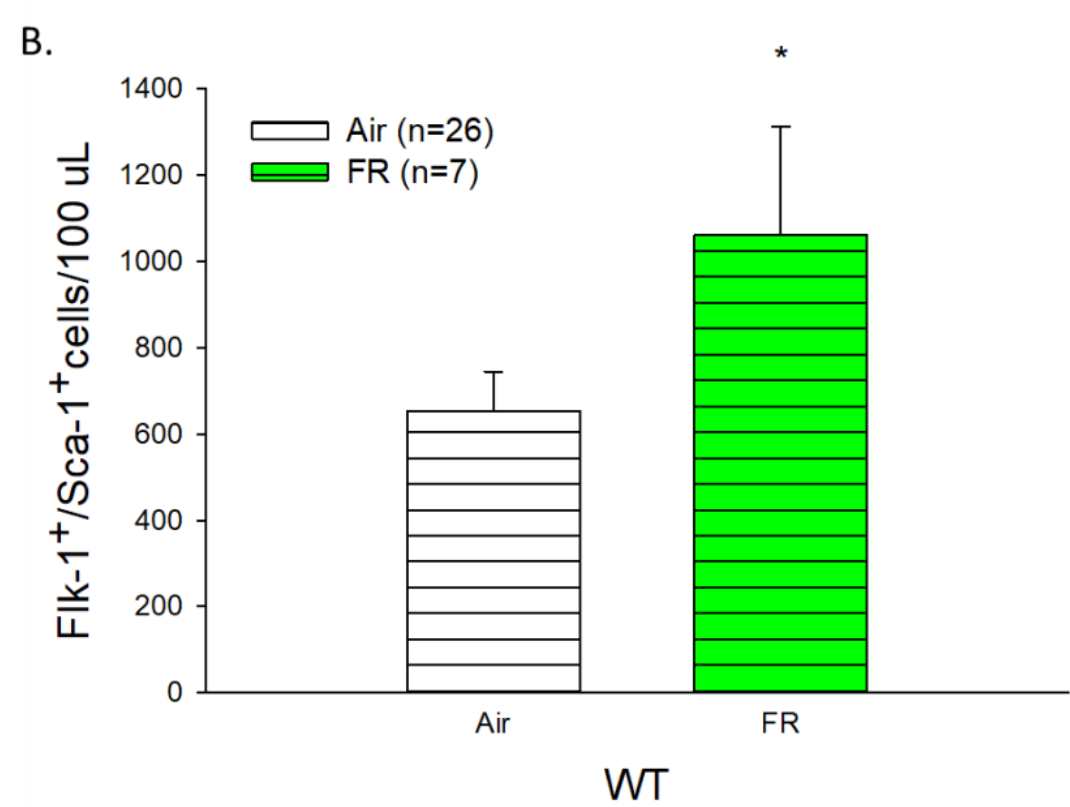

Fig. 5.3. Effects of acute formaldehyde (FR; 5 ppm) exposure on circulating stem cells in male mice. Hematopoietic stem cells (Sca-1+) and circulating angiogenic cells (CACs) were measured by flow cytometry in male C57BL/6J (wild type, WT) mice exposed to HEPA-filtered air or formaldehyde $(5 \mathrm{ppm}, 6 \mathrm{~h} / \mathrm{d})$ for 4 days. After four days of exposure to FR, the levels of circulating Sca-1+ cells were unchanged (A), however, the level of CACs (Flk-1+/Sca-1+ cells) was significantly increased in WT mice $(\mathbf{B}) . \quad$ Values $=$ mean \pm $\mathrm{SE}$ ( $\mathrm{n}=7-26$ mice per group). *, $\mathrm{p}<0.05$ compared with air control. 
A.

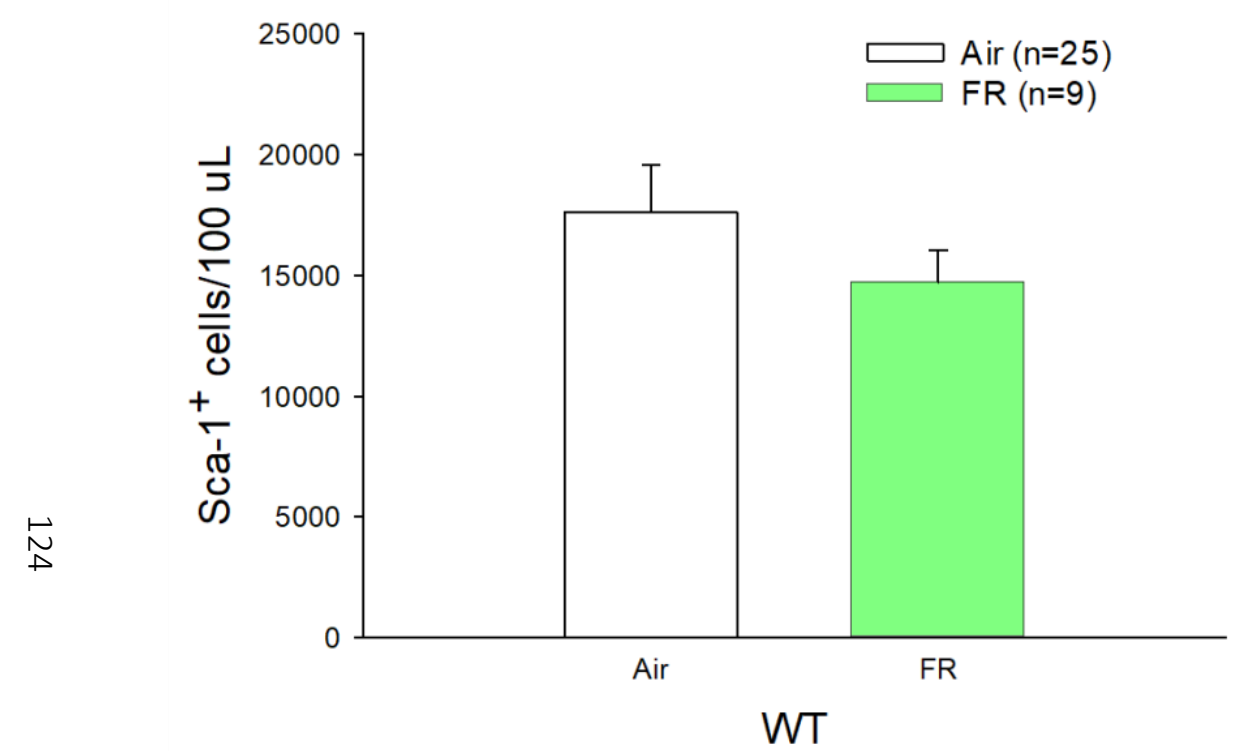

B.

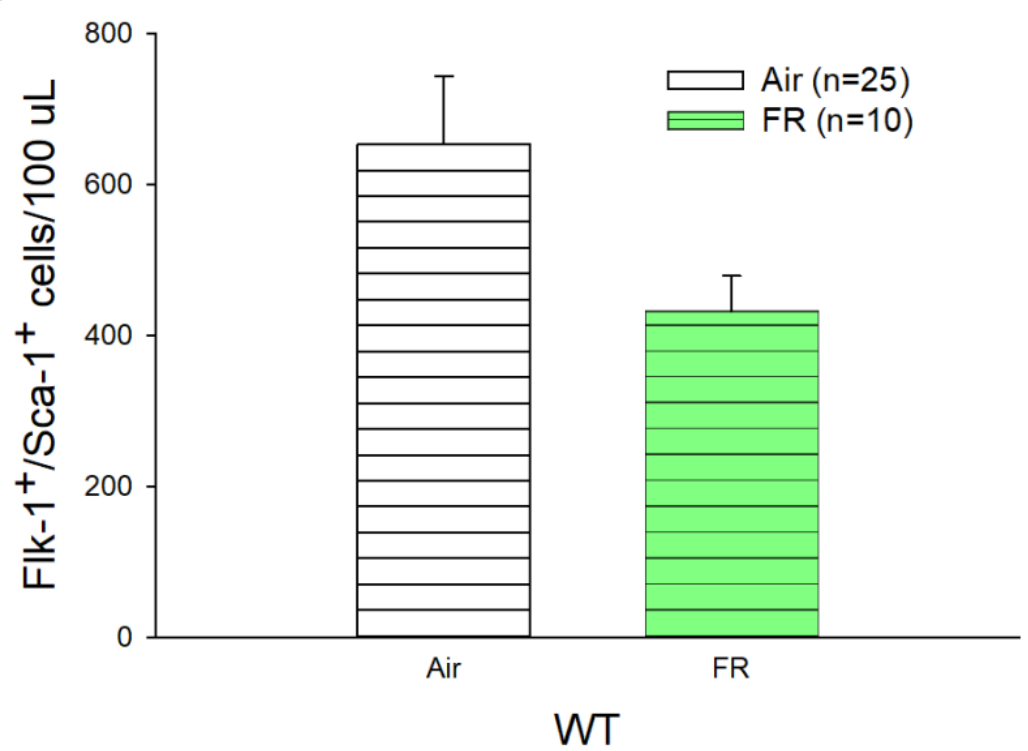

Fig. 5.4. Effects of acute formaldehyde (FR; 2 ppm) exposure on circulating stem cells in male mice. Hematopoietic stem cells (Sca-1+) and circulating angiogenic cells (CACs) were measured by flow cytometry in male C57BL/6J (wild type, WT) mice exposed to HEPA-filtered air or formaldehyde ( 2 ppm, 6h/d) for 4 days. Levels of circulating Sca-1+ cells (A) and CACs (Flk-1+/Sca-1+ cells; B) were unchanged in FR-exposed mice relative to air control mice. Values = mean $\pm \mathrm{SE}$ ( $\mathrm{n}=9-25$ mice per group). 
A.

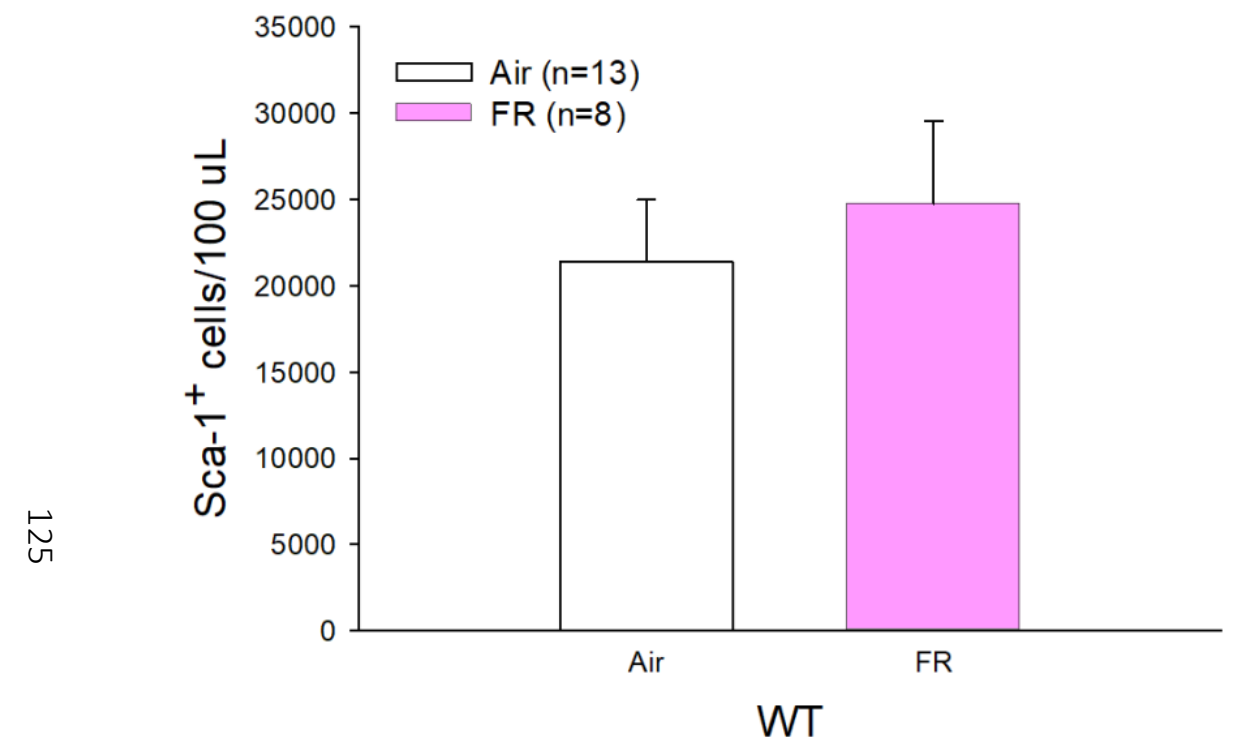

B.

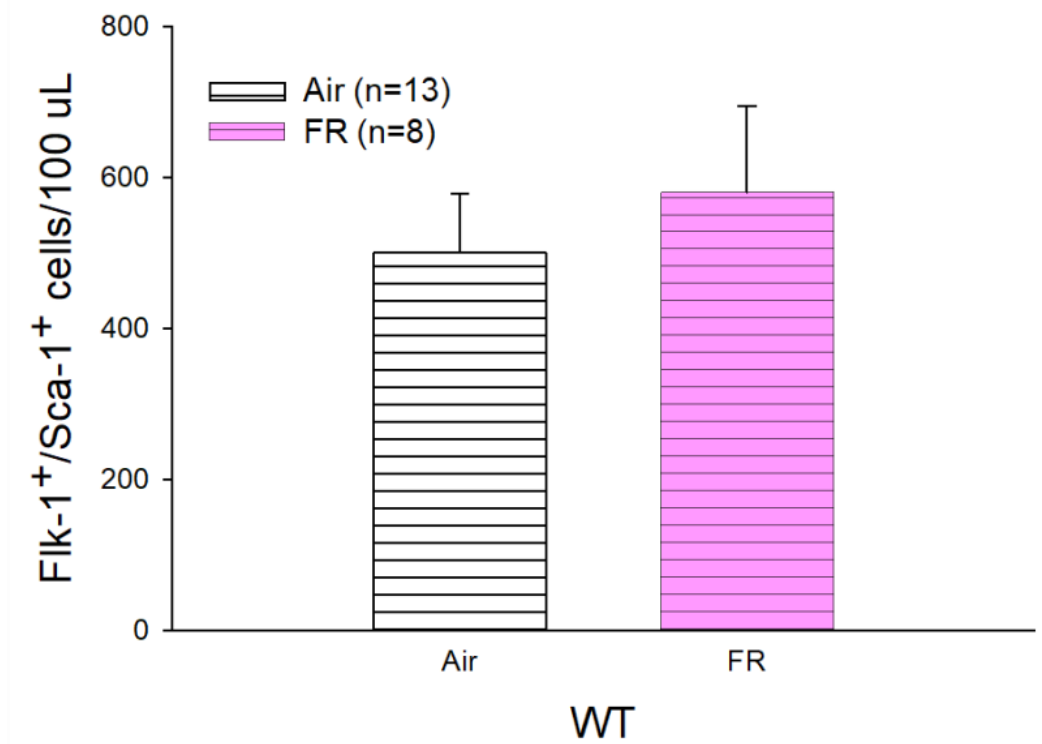

Fig. 5.5. Effects of acute formaldehyde (FR; 2 ppm) exposure on circulating stem cells in female mice. Hematopoietic stem cells (Sca-1+) and circulating angiogenic cells (CACs) were measured by flow cytometry in female C57BL/6J (wild type, WT) mice exposed to HEPA-filtered air or formaldehyde ( 2 ppm, 6h/d) for 4 days. Levels of circulating Sca-1+ cells (A) and CACs (Flk-1+/Sca-1+ cells; B) were unchanged in FR-exposed mice relative to air control mice. Values = mean $\pm \mathrm{SE}$ ( $\mathrm{n}=8-13$ mice per group). 


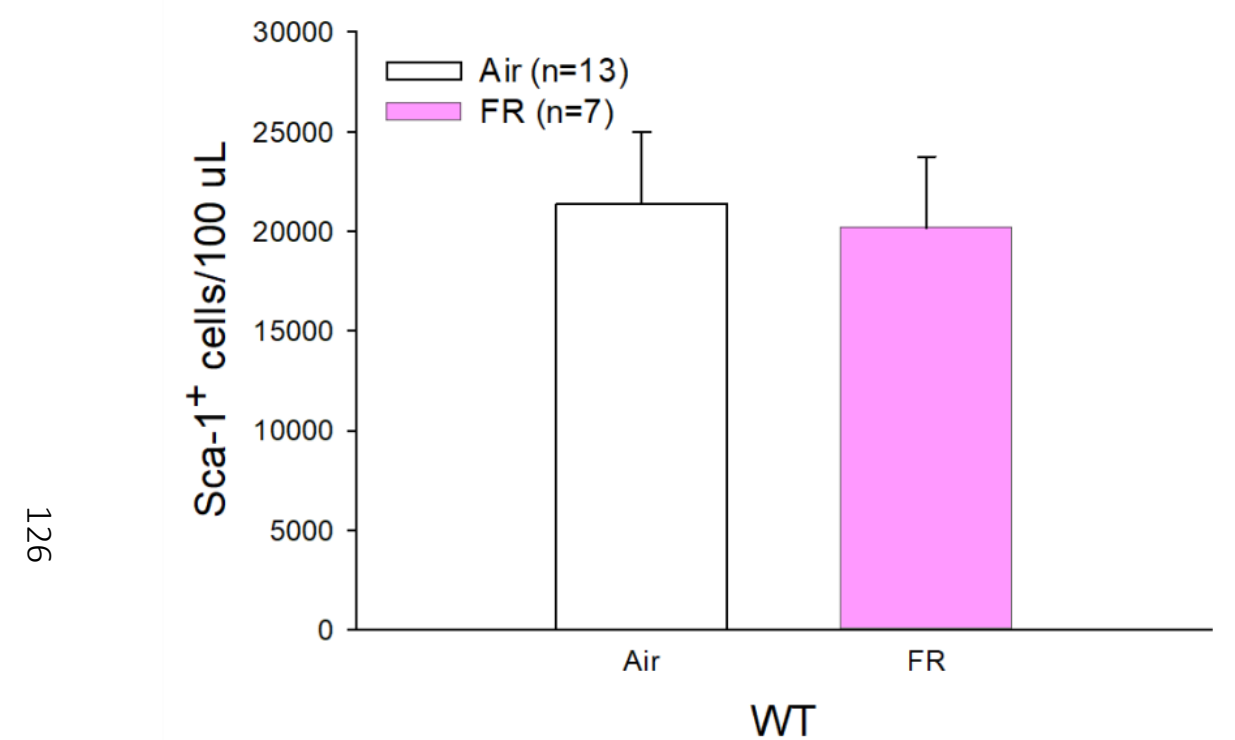

B.

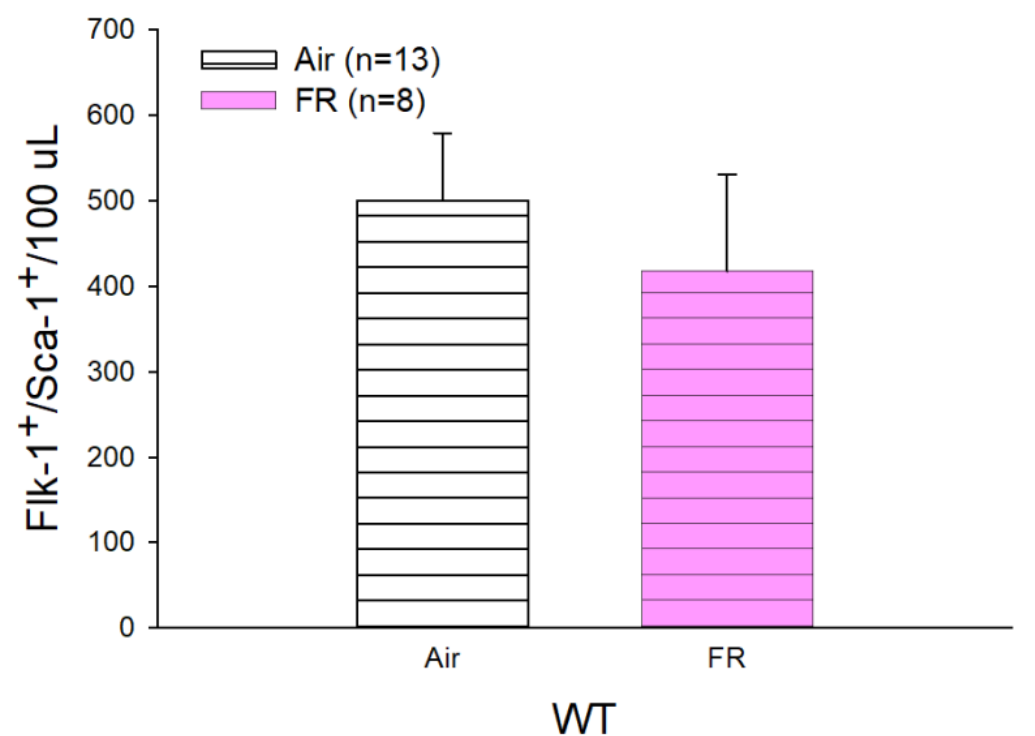

Fig. 5.6. Effects of acute formaldehyde (FR; 5 ppm) exposure on circulating stem cells in female mice. Hematopoietic stem cells (Sca-1+) and circulating angiogenic cells (CACs) were measured by flow cytometry in female C57BL/6J (wild type, WT) mice exposed to HEPA-filtered air or formaldehyde ( 5 ppm, 6h/d) for 4 days. Levels of circulating Sca-1+ cells (A) and CACs (Flk-1+/Sca-1+ cells; B) were unchanged in FR-exposed mice relative to air control mice. Values = mean $\pm \mathrm{SE}$ ( $\mathrm{n}=7-13$ mice per group). 
primary urinary metabolite of AA, has not been validated as a urinary biomarker of tobacco exposure $(431,432)$, but it has been shown to increase with tobacco-product use $(168,293$, 434). To better understand how exposure to AA-alone related to MCS exposure from a known number of cigarettes and to e-cig exposure from known ratios of PG:VG, we measured urinary acetate by UPLC-MS/MS to confirm that the concentration of AA used in our AA-alone exposure was comparable to exposures from real-world sources. For this, male mice were exposed to AA (5 ppm, 6h), MCS (50\% of smoke of $12 \mathrm{KY}$ Reference 3R4F cigarettes $6 \mathrm{~h})$, or PG:VG $(50 \%: 50 \%$ or $30 \%: 70 \%, 6 \mathrm{~h})$, and urine was collected immediately after the exposure sessions. Measures of urinary acetate in AA-, MCS-, and 50\%:50\% PG:VG-exposed mice were not significantly different from baseline during the first three hours post-exposed, but mice exposed to 30\%:70\% PG:VG showed increased levels ( $\mathrm{p}=0.047$ ) (Fig. 5.7). As the levels of acetate in AA-exposed mice were not significantly different from MCS-exposed mice, this likely indicates that the concentration of AA generated for the AA-alone exposure (5 ppm) is similar to the level generated in MCS (Fig. 5.7). Levels of acetate after the overnight (3-16h) collections were elevated compared with levels 0-3h post-exposure with all exposures; these elevations are likely related to generation by food, as mice were given free access to food during this collection period (Fig. 5.7). Additionally, levels of acetate during the overnight collection were significantly higher in 30\%:70\% PG:VG-exposed mice compared with baseline (Fig. 5.7).

\section{Systemic Toxicity of Two-Week Exposure to Inhaled Acetaldehyde}

Because of the potential for MCS to significantly increase levels of AA exposure $(107,118)$, we tested for changes in systemic and hematological biomarkers in mice exposed to AA (5 ppm). Two weeks of exposure had limited to modest effects on organ 


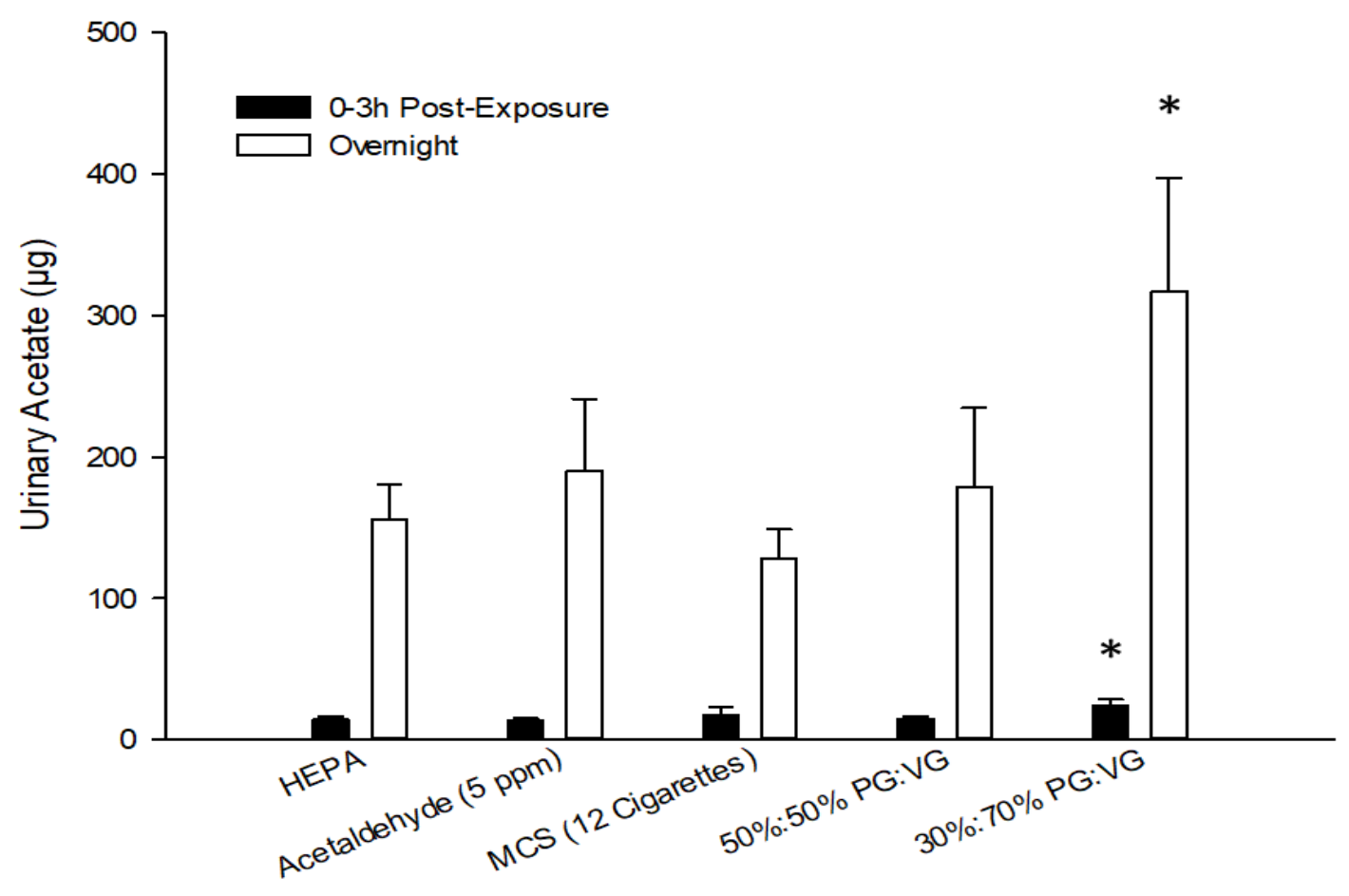

Fig. 5.7. Urinary acetate levels in acetaldehyde- or tobacco product-exposed male mice. Male mice were exposed (6h) to acetaldehyde (5 ppm), mainstream cigarette smoke (MCS; 50\% smoke of 12 cigarettes), or propylene glycol:vegetable glycerin (PG:VG; $50 \%: 50 \%$ or $30 \%: 70 \%, 6 \mathrm{~h})$ after which urine was collected at $0-1,1-2,2-3$, and $3-16 \mathrm{~h}$ (overnight) and acetate quantified. Values are mean $\pm \mathrm{SE}(\mathrm{n}=4-12$ mice per group). *, $\mathrm{p}<0.05$ compared with HEPA. 
and body weights (or ratios), $\mathrm{CBC}$, or plasma markers (Tables $5.5 \& 5.6$ ). For example, exposure to 5 ppm AA caused a modest decrease in final body weight $(-4 \% ; \mathrm{p}=0.09)$ but a slight increase in the heart/body weight ratio $(+8 \% ; \mathrm{p}=0.02)($ Table 5.5$)$, and although low plasma volumes limited many other measures of systemic toxicity, AA-exposed mice showed a modest decrease in cholesterol $(-16 \% ; \mathrm{p}=0.05)$ compared with their air-exposed control counterparts (Table 5.6).

\section{Two-Week Exposure to Acetaldehyde and Circulating Angiogenic Cells}

Despite the minimal systemic effects noted in AA-exposed male mice ( $5 \mathrm{ppm} ; 2$ weeks), AA induced a modest increase in the level of Sca- $1+$ cells $(+58 \% ; \mathrm{p}=0.06)$, although CACs were not significantly changed (Fig. 5.8A \& B).

\section{Systemic Toxicity of Acute Exposure to Inhaled Acetaldehyde}

Although two weeks of exposure to AA (5 ppm) has minimal systemic toxicity (decreased body weight, decreased cholesterol, increased neutrophils), we performed acute (4d) AA exposures to test whether acute CR exposure would induce rapid toxicity that was otherwise compensated for in a longer setting. Male mice acutely exposed to AA (5 ppm) showed an increased body weight $(+11 \%$; $\mathrm{p}=0.01)$, a modestly increased heart/body weight ratio $(+9 \% ; \mathrm{p}=0.06)$, and a modestly decreased lung/body weight ratio $(-6 \% ; \mathrm{p}=0.06)$ (Table 5.5). Plasma levels of cholesterol, HDL, LDL, and albumin were unchanged with acute exposure, while levels of total protein were modestly decreased $(-4 \% ; \mathrm{p}=0.07)$ (Table 5.5). Acute (4d) exposure to AA (5 ppm) induced a number of hematological changes, decreasing red blood count, hemoglobin, and hematocrit levels $(-14 \%, \mathrm{p}=0.01 ;-16 \%$, $\mathrm{p}=0.05$; and $-12 \%, \mathrm{p}=0.03$, respectively), while increasing mean platelet volume $(+6$; $\mathrm{p}=0.04)$ (Table 5.6). No changes in body weight, organ/body weight ratios, or CBC were 
Table 5.5. Systemic parameters of male C57BL/6 mice acutely exposed to either air or acetaldehyde (AA; 5 ppm).

\begin{tabular}{|c|c|c|c|}
\hline \multicolumn{4}{|c|}{ Exposure } \\
\hline \multirow[b]{2}{*}{ Variable } & \multirow[b]{2}{*}{ Air Control } & \multicolumn{2}{|c|}{ AA (5 ppm) } \\
\hline & & 4 days & 2 weeks \\
\hline Final BWT (g) & $28 \pm 0$ & $31 \pm 1 *$ & $27 \pm 1 \#$ \\
\hline Heart/BWT (mg/g) & $4.8 \pm 0$ & $5.3 \pm 0.2 \#$ & $5.2 \pm 0.2 *$ \\
\hline Lung/BWT (mg/g) & $5.5 \pm 0$ & $5.2 \pm 0.1 \#$ & $5.7 \pm 0.2$ \\
\hline Liver/BWT (mg/g) & $40.0 \pm 1$ & $38.4 \pm 1.3$ & $41.9 \pm 1.1$ \\
\hline Kidney/BWT (mg/g) & $12.9 \pm 1$ & $13.2 \pm 0.5$ & $12.7 \pm 0.3$ \\
\hline \multicolumn{4}{|c|}{ Plasma Measurements } \\
\hline Cholesterol (mg/dL) & $65.05 \pm 3.46$ & $72.37 \pm 4.30$ & $54.84 \pm 3.35^{*}$ \\
\hline HDL (mg/dL) & $41.68 \pm 4.60$ & $48.86 \pm 4.24$ & $35.24 \pm 1.06$ \\
\hline LDL (mg/dL) & $5.49 \pm 0.91$ & $5.71 \pm 1.07$ & N/A \\
\hline Triglycerides (mg/dL) & $49.76 \pm 1.71$ & N/A & $52.19 \pm 2.07$ \\
\hline Albumin (g/dL) & $2.94 \pm 0.05$ & $2.87 \pm 0.04$ & $2.91 \pm 0.06$ \\
\hline Total Protein (g/dL) & $4.98 \pm 0.08$ & $4.76 \pm 0.06 \#$ & $4.85 \pm 0.12$ \\
\hline
\end{tabular}

Values = mean \pm SE (n=5-10 mice per group); Abbr.: AA, acetaldehyde; BWT, body weight; HDL, high-density lipoprotein; LDL, low-density lipoprotein; *, $\mathrm{p}<0.05$ compared with air control; \#, $0.05 \leq \mathrm{p} \leq 0.10$ compared with air control. 
Table 5.6. Hematological measures of male C57BL/6 mice acutely exposed to either air or acetaldehyde (AA; 5 ppm).

\begin{tabular}{|c|c|c|c|}
\hline \multirow{2}{*}{ Hematological Measurements } & \multicolumn{3}{|c|}{ Exposure } \\
\hline & Air Control & \multicolumn{2}{|c|}{ AA (5 ppm) } \\
\hline White Blood Cell $(\mathrm{K} / \mathrm{uL})$ & $1.10 \pm 0.15$ & $1.24 \pm 0.19$ & $1.29 \pm 0.18$ \\
\hline Lymphocytes (K/uL) & $0.74 \pm 0.11$ & $0.73 \pm 0.10$ & $0.66 \pm 0.06$ \\
\hline Monocytes (K/uL) & $0.04 \pm 0$ & $0.05 \pm 0.01$ & $0.03 \pm 0.01$ \\
\hline Eosinophils (K/uL) & $0 \pm 0$ & $0 \pm 0$ & $0 \pm 0$ \\
\hline Hemoglobin (g/dL) & $12.5 \pm 0.3$ & $10.5 \pm 0.8^{*}$ & $13.2 \pm 0.1$ \\
\hline Hematocrit (\%) & $35.6 \pm 0.4$ & $31.5 \pm 2.2 *$ & $35.9 \pm 0.4$ \\
\hline Mean Corpuscular Volume (fL) & $40.7 \pm 0.3$ & $41.8 \pm 0.2$ & $40.4 \pm 0.3$ \\
\hline Mean Corpuscular Hemoglobin (pg) & $14.2 \pm 0.2$ & $13.9 \pm 0.2$ & $14.8 \pm 0.1$ \\
\hline Mean Corpuscular Hemoglobin Concentration (g/dL) & $35.0 \pm 0.8$ & $33.1 \pm 0.4$ & $36.6 \pm 0.3$ \\
\hline
\end{tabular}

Values $=$ mean $\pm \mathrm{SE}(\mathrm{n}=5-10$ mice per group); Abbr.: AA, acetaldehyde; $*, \mathrm{p}<0.05$ compared with air control; \#, $0.05 \leq \mathrm{p} \leq 0.10$ compared with air control. 
A.

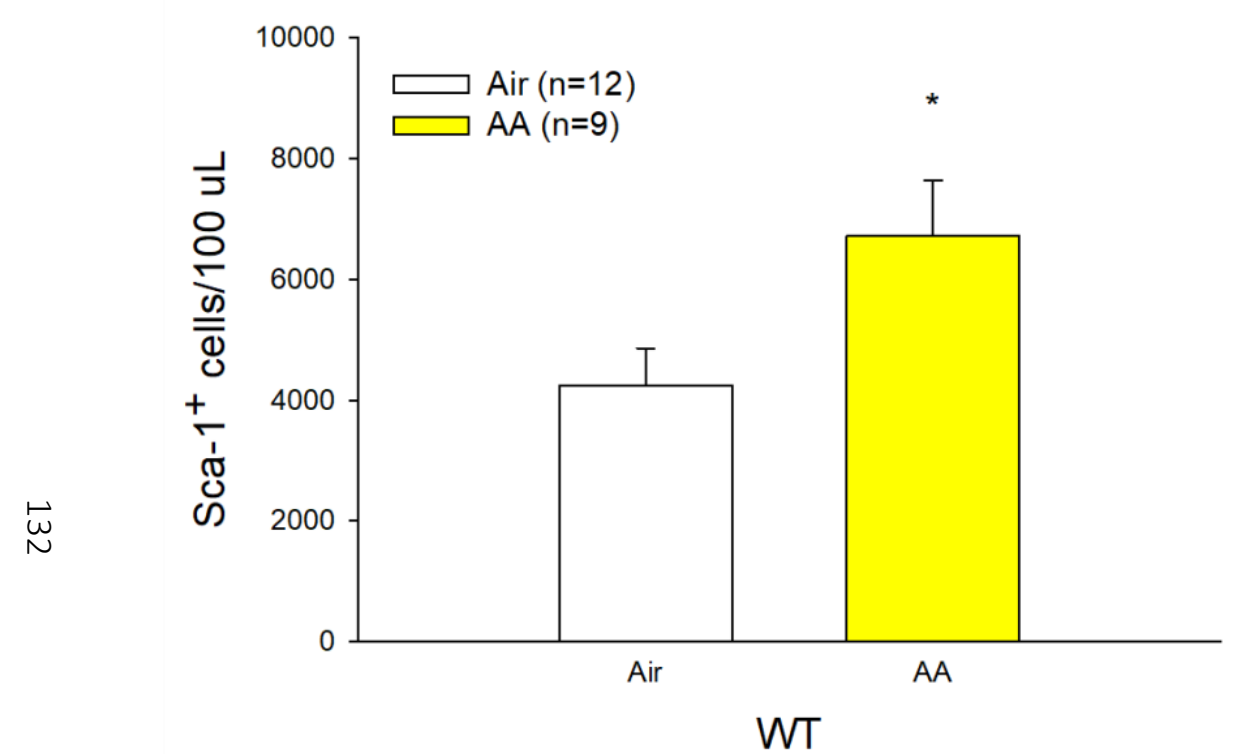

B.

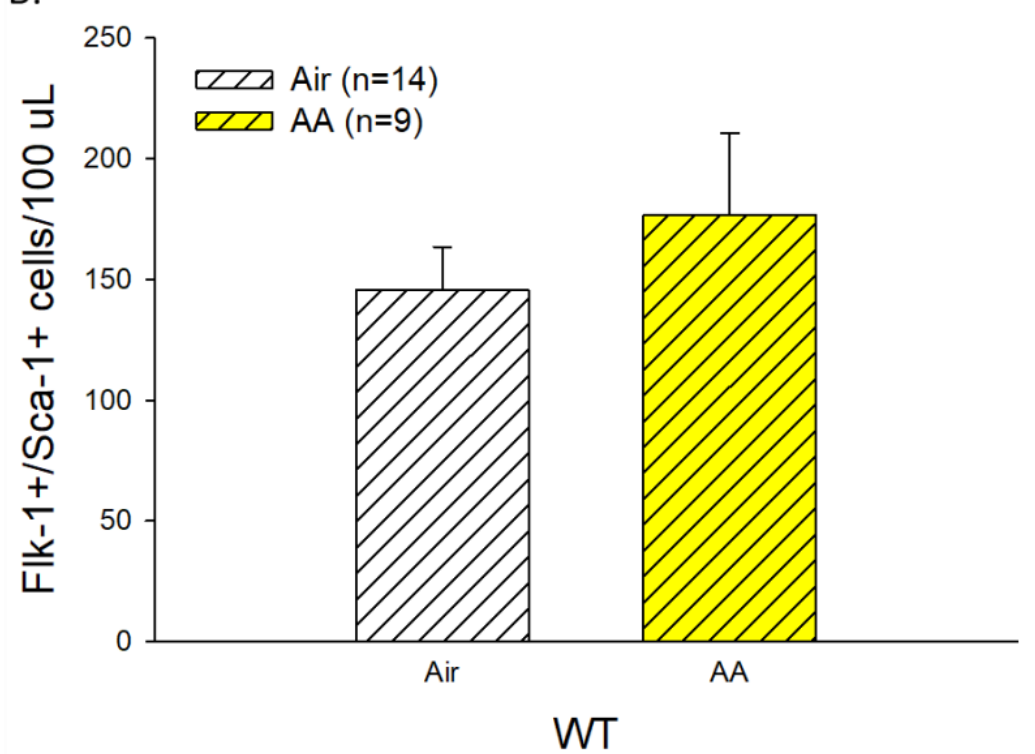

Fig. 5.8. Effects of two-week acetaldehyde (AA; 5 ppm) exposure on circulating stem cells in male mice. Hematopoietic stem cells (Sca-1+) and circulating angiogenic cells (CACs) were measured by flow cytometry in male C57BL/6J (wild type, WT) male mice exposed to HEPA-filtered air or acetaldehyde (5 ppm, 6h/d) for two weeks. After two weeks of exposure to AA, the levels of circulating Sca-1+ cells were significantly increased in WT mice (A), however, no change in the level of CACs (Flk-1+/Sca-1+ cells) was detected relative to air-exposed control mice $(\mathbf{B})$. Values $=$ mean $\pm \mathrm{SE}$ ( $\mathrm{n}=9-14$ mice per group). *, $\mathrm{p}<0.05$ compared with air control. 
observed in female mice exposed for 4 days to 5 ppm AA (Table 5.7); low plasma volumes prevented additional measures of systemic toxicity.

\section{Acute Exposure to Acetaldehyde and Circulating Angiogenic Cells}

Neither male nor female mice acutely exposed to AA ( $5 \mathrm{ppm}$; 4d) showed changes in the levels of CACs or Sca-1+ cells (Figs. 5.9A \& B, 5.10A \& B).

\section{Discussion}

Exposures to FR and AA have been shown to have negative cardiovascular effects, and they are known to be significant toxins in MCS (96). Although exposure to these saturated aldehydes is fairly ubiquitous, exposure to MCS or secondhand smoke can significantly increase the level of exposure (117). Likewise, new electronic tobacco products including e-cigs, which have become increasingly popular among adolescents $(182,183)$, have been shown to produce high levels of FR and AA $(96,180)$, potentially subjecting a new segment of the population to acute, high-level exposures of these compounds. There are still gaps in our understanding of how saturated aldehydes such as FR and AA contribute to the endothelium dysfunction and subsequent CVD linked to MCS and e- cig aerosol exposures. The goal of the current studies was to measure biomarkers of systemic and vascular injury in mice exposed to FR or AA. Furthermore, these studies explored the dependence of concentration, duration, and sex on these outcomes.

Measures of formate, the urinary metabolite of FR, showed that FR generation may differ significantly between tobacco products. Levels of formate in MCS-exposed mice were significantly higher than levels after exposure to $5 \mathrm{ppm}$ FR. Levels of formate differed even between different compositions of PG:VG, with 50\%:50\% PG:VG, the composition used in blu Plus+ e-cigs, generating higher levels of FR than 30\%:70\% 
Table 5.7. Systemic changes in female C57BL/6 mice acutely exposed to either air or acetaldehyde (AA; 5 ppm).

\begin{tabular}{|l|c|c|}
\hline \multicolumn{1}{|c|}{ Variable } & \multicolumn{2}{c|}{ Exposure } \\
\hline \multicolumn{1}{|c|}{ Air Control } & AA (5 ppm) \\
\hline Final BWT (g) & $21 \pm 0$ & $23 \pm 1$ \\
\hline Heart/BWT (mg/g) & $5.0 \pm 0.2$ & $5.0 \pm 0.2$ \\
\hline Lung/BWT (mg/g) & $7.0 \pm 0.2$ & $6.8 \pm 0.2$ \\
\hline Liver/BWT (mg/g) & $44.8 \pm 0.9$ & $46.1 \pm 1.8$ \\
\hline Kidney/BWT (mg/g) & $12.6 \pm 0.4$ & $13.0 \pm 0.3$ \\
\hline White Blood Cell (K/uL) & & \\
\hline Neutrophils (K/uL) & $1.60 \pm 0.34$ & $1.15 \pm 0.17$ \\
\hline Lymphocytes (K/uL) & $0.45 \pm 0.14$ & $0.28 \pm 0.03$ \\
\hline Monocytes (K/uL) & $1.10 \pm 0.19$ & $0.82 \pm 0.14$ \\
\hline Eosinophils (K/uL) & $0.04 \pm 0.01$ & $0.04 \pm 0.01$ \\
\hline Basophils (K/uL) & $0 \pm 0$ & $0 \pm 0$ \\
\hline Red Blood Cell (M/uL) & $0 \pm 0$ & $0 \pm 0$ \\
\hline Hemoglobin (g/dL) & $9.23 \pm 0.05$ & $9.32 \pm 0.11$ \\
\hline Hematocrit (\%) & $11.9 \pm 0.1$ & $11.7 \pm 0.2$ \\
\hline Mean Corpuscular Volume (fL) & $38.5 \pm 0.3$ & $38.1 \pm 0.5$ \\
\hline Mean Corpuscular Hemoglobin (pg) & $41.7 \pm 0.3$ & $40.9 \pm 0.7$ \\
\hline Mean Corpuscular Hemoglobin Concentration (g/dL) & $13.0 \pm 0.1$ & $12.5 \pm 0.3$ \\
\hline Red Cell Distribution Width (\%) & $31.0 \pm 0.3$ & $30.6 \pm 0.4$ \\
\hline Platelets (K/uL) & $17.7 \pm 0.2$ & $17.6 \pm 0.1$ \\
\hline Mean Platelet Volume (fL) & $695 \pm 36$ & $680 \pm 22$ \\
\hline & $4.4 \pm 0.1$ & $4.4 \pm 0.1$ \\
\hline
\end{tabular}

Values $=$ mean \pm SE ( $\mathrm{n}=5-6$ mice per group); Abbr.: AA, acetaldehyde; $\mathbf{B W T}$, body weight. 
A.

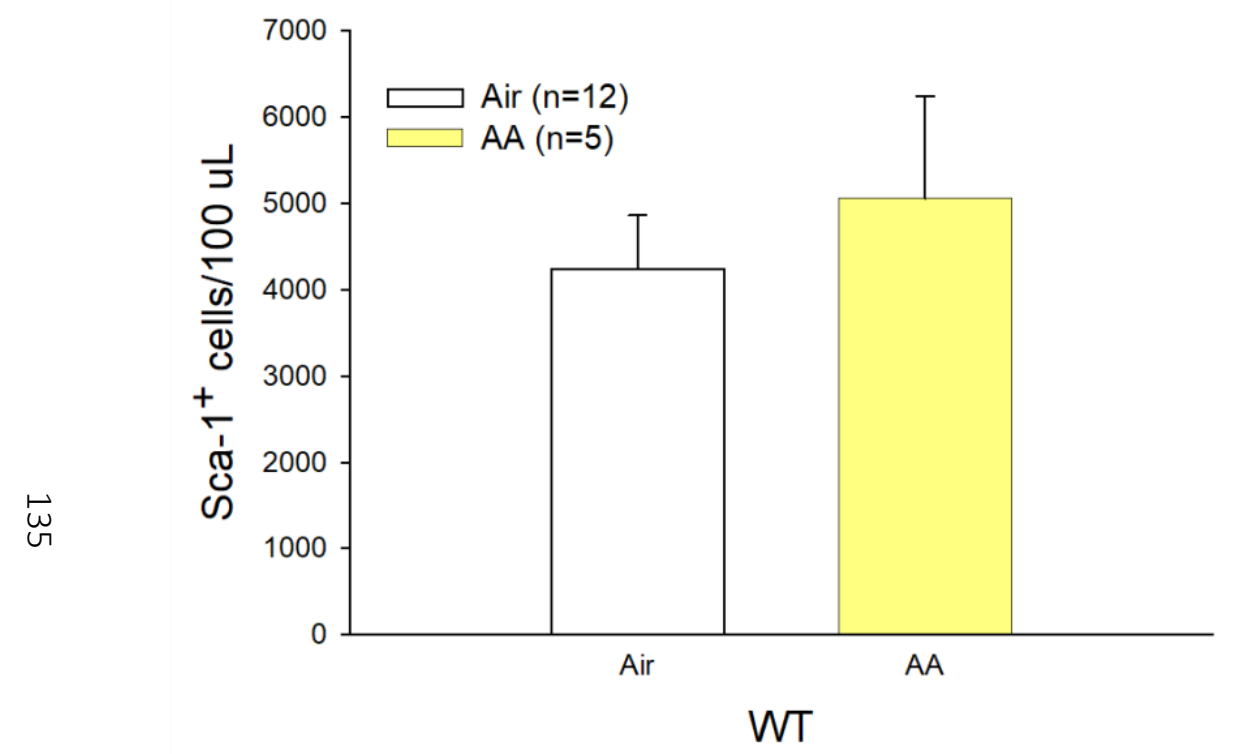

B.

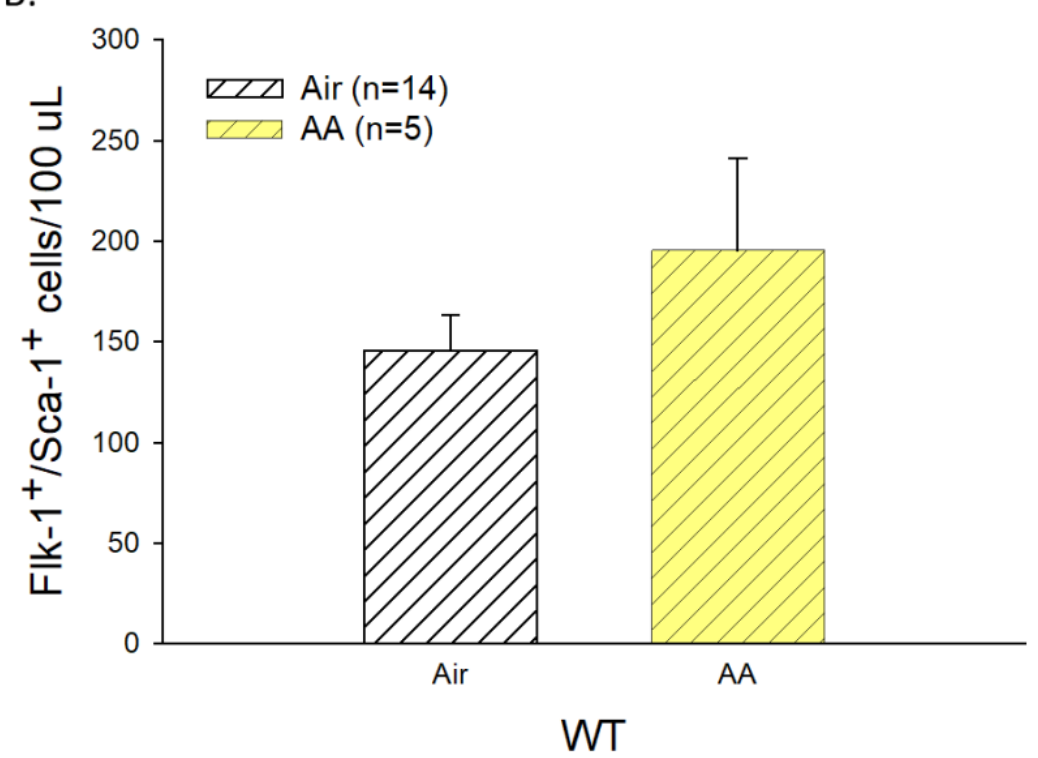

Fig. 5.9. Effects of acute acetaldehyde (AA; 5 ppm) exposure on circulating stem cells in male mice. Hematopoietic stem cells (Sca-1+) and circulating angiogenic cells (CACs) were measured by flow cytometry in male C57BL/6J (wild type, WT) mice exposed to HEPA-filtered air or acetaldehyde (5 ppm, 6h/d) for 4 days. Levels of circulating Sca-1+ cells (A) and CACs (Flk-1+/Sca-1+ cells; B) were unchanged in AA-exposed mice relative to air control mice. Values = mean $\pm \mathrm{SE}(\mathrm{n}=5-14$ mice per group). 
A.

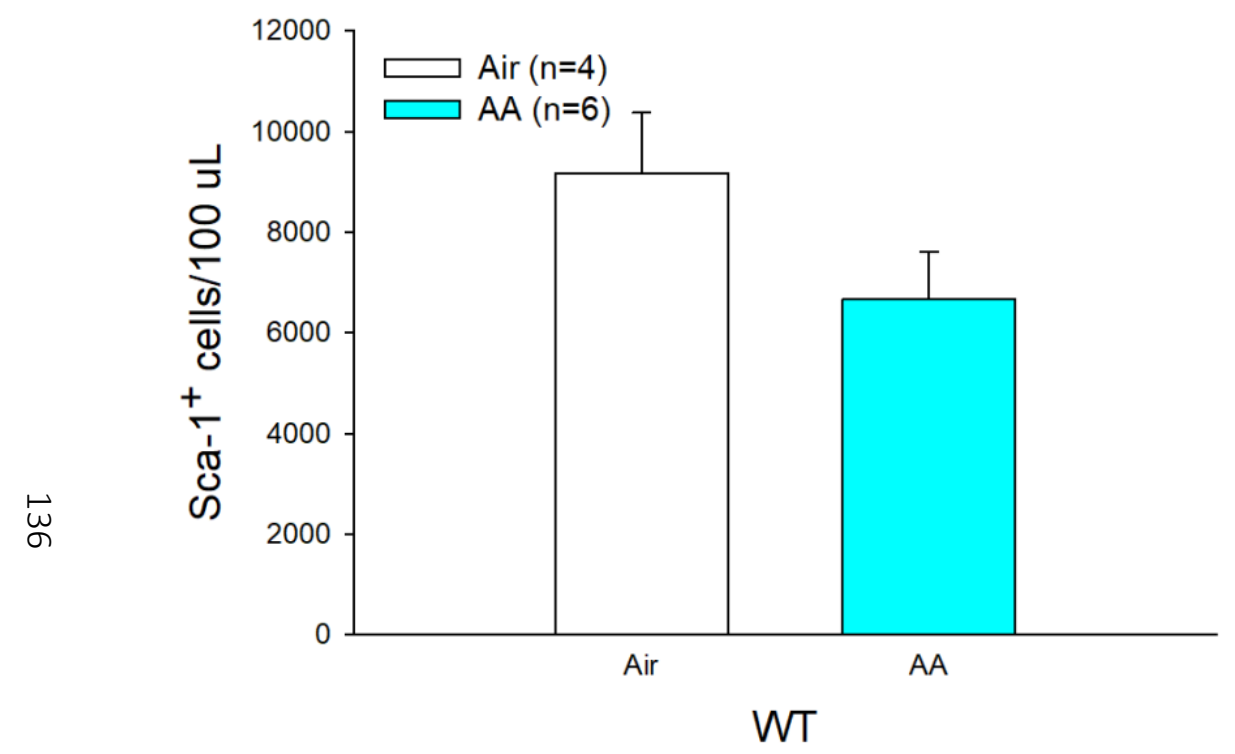

B.

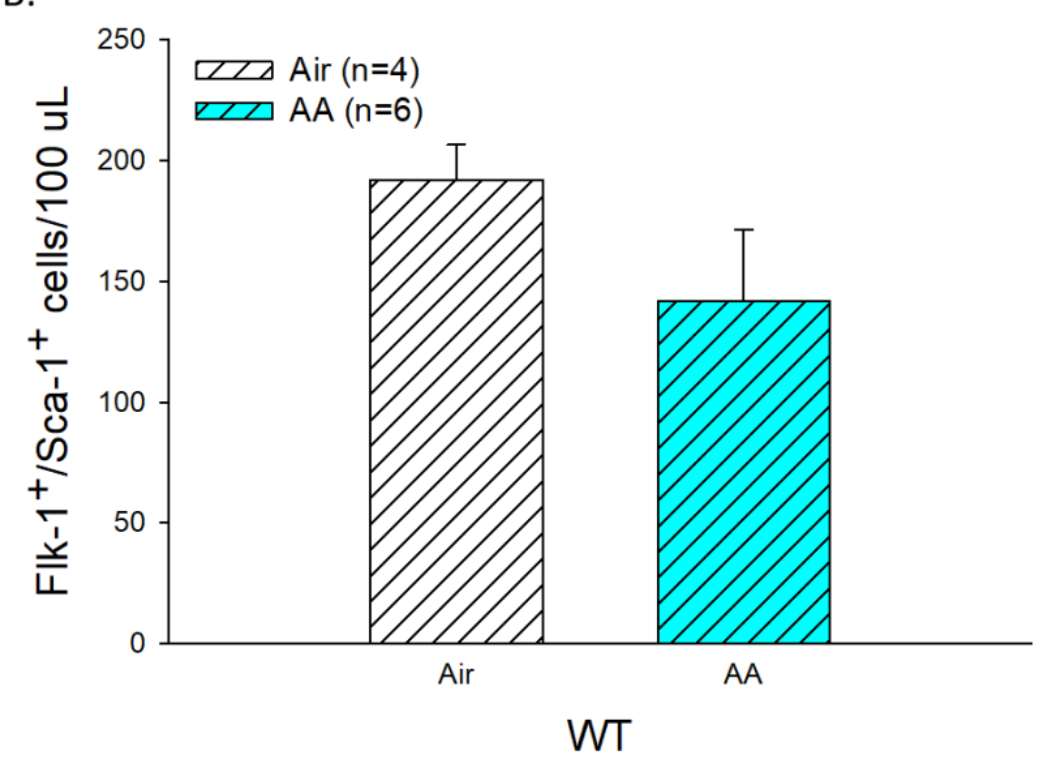

Fig. 5.10. Effects of acute acetaldehyde (AA; 5 ppm) exposure on circulating stem cells in female mice. Hematopoietic stem cells (Sca-1+) and circulating angiogenic cells (CACs) were measured by flow cytometry in female C57BL/6J (wild type, WT) mice exposed to HEPA-filtered air or acetaldehyde $(5 \mathrm{ppm}, 6 \mathrm{~h} / \mathrm{d})$ for 4 days. Levels of circulating Sca-1+ cells (A) and CACs (Flk-1+/Sca-1+ cells; B) were unchanged in AA-exposed mice relative to air control mice. Values = mean $\pm \mathrm{SE}$ ( $\mathrm{n}=7-13$ mice per group). 
PG:VG, the composition used in JUUL devices, although this was not a significant difference. As formate measured in MCS-exposed mice was not significantly different than measured in PG:VG-exposed mice, the current data would suggest that e-cigs do not generate more FR than traditional cigarettes. Additionally, the data suggest that, as there was no significant increase in formate with an increased level of VG in the tested PG:VG ratios, VG is not the primary producer of FR in e-cigs. Previous data, however, have shown that the generation of FR does in fact differ between MCS and e-cigs. Ogunwale et al. (96) report significantly higher levels of FR generation in MCS than in aerosol from a number of different blu cartridges, although levels of FR similar to levels in MCS were measured in a handful of different e-cig liquids outside of the blu brand. As our study only exposed mice to $50 \%$ of smoke from $12 \mathrm{KY} 3 \mathrm{R} 4 \mathrm{~F}$ cigarettes, it may be that the amount of FR generated was not sufficient to show a significant increase over levels from PG:VG exposure despite showing a significant increase compared with baseline. Additional studies exposing mice to $100 \%$ of the smoke from the same number of cigarettes may show results similar to those found in the literature. Furthermore, measures of aldehyde generation in PG- and VG-alone showed higher levels of FR per puff with VG-alone compared with PG-alone (168). These differences may indicate that PG and VG together are capable of generating FR at different concentrations than generated by the individual humectants.

Measures of urinary acetate, the urinary metabolite of AA, also differed between MCS and PG:VG exposures, with levels measured in MCS-exposed mice similar to levels in 50\%:50\% PG:VG-exposed mice but lower than those in 30\%:70\% PG:VG-exposed mice. This again seems to contradict what has previously been reported concerning levels 
of AA in MCS and e-cigs (96), but the similar levels measured in 50\%:50\% and 30\%:70\% PG:VG-exposed mice is comparable to the similar levels of acetate measured after PG- and VG-alone exposures (168). Exposure to $5 \mathrm{ppm}$ AA produced urinary acetate levels similar to those measured in the 50\%:50\% PG:VG-exposed mice, likely indicating that the level of AA generated by the equivalent blu Plus+ e-cig devices is around $5 \mathrm{ppm}$.

Studies have shown that additional factors such as the voltage of the e-cig device can influence aldehyde generation $(96,208)$. Additionally, flavored e-cig liquids have been shown to produce high levels of both FR and AA $(213,435)$, and as the use of ENDS has grown increasingly customizable (436), accurate measurements of aldehyde generationas well as generation of other harmful constituents-have become more difficult. This makes research on the generation of aldehydes by individual components of e-cigs even more imperative, as a baseline understanding of potential product harm must be established in order to understand how the more complex web of factors contributes to e-cig-related health effects. As research into e-cigs and their health effects is still a fairly new field, it is not unexpected that studies may produce varying results, especially as different products, flavors, and humectants may be used for testing. This emphasizes the complexity of e-cigs as well as the need for more research. Nonetheless, our studies show that the concentrations used for our aldehyde-alone exposures produce urinary metabolites at similar levels as produced in MCS- and PG:VG-exposed mice, justifying the use of these concentrations as relevant real-world exposure levels.

Overall, exposures to FR seemed to produce little to moderate systemic effects. Although some significant changes were seen in response to acute exposure to FR at 1, 2, and $5 \mathrm{ppm}$, these changes are unlikely to be physiologically significant, with changed 
measurements typically still within the normal physiological range for specific biomarkers. However, two weeks of exposure to FR $(5 \mathrm{ppm})$ produced significant changes in hematological measures; two-week-exposed male mice showed significant decreases in leukocytes and in platelets, changes which have been previously reported $(120,121)$. Interestingly, after two weeks, we saw an increase in red blood cell count rather than a decrease as reported in the literature (120), but a significant decrease in red blood cells was seen after four days of exposure at the same level $(5 \mathrm{ppm})$. These results could indicate that FR causes a decrease in red blood cells early on but that homeostatic mechanisms were able to compensate after a longer exposure duration. A similar increase was noted in rats exposed to high-level FR (437), although the authors note that the increased levels were not outside the expected range for that strain of rat and were therefore uncertain of its clinical relevance. Likewise, our values remain within the normal range for this strain of mice, so while the values may not indicate physiological significance, this pattern may point towards changes in red blood cell production that could contribute to cardiovascular injury or disease development. Hemoglobin showed a similar pattern of change between four days and two weeks of exposure at 5 ppm FR.

Biomarkers of vascular injury showed no change after four days of FR (2 ppm), but CACs (Flk-1+/Sca-1+ cells) were significantly increased after four days of FR at a higher concentration (5 ppm), and there was also a trend towards increased levels of Sca-1+ cells. After two weeks of exposure ( $5 \mathrm{ppm})$, however, both cell types were decreased, which may indicate that there was significant injury early on which necessitated increased levels of CACs for repair but that these increased levels could not be maintained to repair consistent vascular injury occurring over a longer exposure period. CACs play a role in maintaining 
proper endothelium function $(62,63)$, and decreased levels of these cells can allow for the development of endothelium dysfunction $(66,70)$. Exposure to other aldehydes such as acrolein $(71,74,151)$ as well as MCS itself $(72,318)$ have also been shown to decrease the levels and function of CACs and contribute to endothelium dysfunction, although the shorter time frame in which these effects are produced likely indicates that acrolein is more toxic for these cells than FR. Wheat et al. (74) have shown that acrolein decreases levels of CACs in the blood through the inhibition of vascular endothelial growth factor-induced mobilization of these cells from the bone marrow; future studies of FR exposed should examine levels of CACs in the bone marrow to determine whether this aldehyde's effect on CACs is the result of this mechanism. Nonetheless, the decrease in CACs noted after two weeks of exposure may indicate that FR is capable of inducing endothelium dysfunction, which has been previously suggested (438). However, ex vivo studies in blood vessels did not observe evidence of endothelium dysfunction even in response to high levels of FR (127). This discrepancy could indicate that the effect of FR on endothelium function varies between intact animals and ex vivo vessels, but more studies are needed to better understand this relationship.

Exposure to AA (5 ppm, 4 days and 2 weeks) showed minimal systemic and hematological effects. Unlike FR, AA-exposed mice showed no significant changes in overall leukocyte counts, and although mice exposed to AA for four days showed decreases in red blood cell count, hemoglobin, and hematocrit, these changes were not seen after two weeks of exposure. Two weeks of exposure did cause a significant increase in Sca-1+ cells, but CACs remained unchanged. One study has found a link between AA and the potential development of endothelium dysfunction (439), but this hypothesis is still largely 
unexplored. Although our findings do not suggest that AA is linked to the development of endothelium dysfunction, studies with a larger number of mice and for longer durations would allow for a more definitive clarification of the relationship between AA and endothelium dysfunction.

We also sought to determine whether saturated aldehyde-induced effects on systemic and hematological biomarkers and CACs are sex-dependent. Like their male counterparts, female WT mice exposed to FR (2 or 5 ppm; 4d) or AA (5 ppm; 4d) showed minimal cardiovascular effects. The few significant changes noted in response to FR exposure (2 or $5 \mathrm{ppm}$ ) are not likely to be physiologically significant, and no changes were noted in AA-exposed mice. Likewise, neither FR nor AA exposure in female mice induced changes in CACs. The difference in outcomes between male and female mice exposed to FR or AA at the same concentration and for similar durations may indicate that the aldehyde-induced cardiovascular changes are sex-dependent, particularly the FR-induced effects in CACs, although more robust studies are needed to truly make this determination. Although a small number of studies have reported sex differences in response to other aldehydes $(269,440)$, sex-related differences in acetaldehyde exposures have mainly been explored in relation to AA ingestion rather than inhalation (441-444), and of the historical studies examining long-term effects of formaldehyde exposure (445-449), only Maronpot et al. (445) report significant differences between males and females. Our studies therefore represent novel observations regarding the potential sex-dependence of full-body saturated aldehyde inhalation exposure on cardiovascular effects in mice, especially in regard to differences in biomarkers of vascular injury in response to FR exposure.

In summary, the studies presented in this chapter have demonstrated the importance 
of research into the saturated aldehydes FR and AA as potential contributors of systemic and vascular injury in response to inhalation exposure. Levels of these constituents were measured at levels comparable to those generated in response to exposure from MCS and varying compositions of $\mathrm{PG}: \mathrm{VG}$, indicating their real-world relevance in determining the health effects of e-cig use. Two weeks of exposure to FR (5 ppm) in particular induced significant systemic changes that confirm the results of previous studies and implicate FR as a promoter of CVD. This exposure also induced changes in CACs that suggest FR may play a role in the development of endothelium dysfunction in vivo. Furthermore, the CAC effects induced by FR seem to be sex-dependent, although additional studies are needed to confirm this finding. As smoking conventional cigarettes remains a global health threat and as the number of e-cig users continues to increase, these studies provide novel insight into the role of saturated aldehydes in the development of CVD and suggest differences between male and females that could play an important role in the therapeutic treatment of FR exposure. 


\section{CHAPTER VI}

\section{SYSTEMIC, HEMATOLOGICAL, AND ENDOTHELIUM-RELATED EFFECTS OF ACROLEIN AND CROTONALDEHYDE IN MICE}

\section{A. Introduction}

The unsaturated aldehydes acrolein and crotonaldehyde (CR) are classified as eye, nose, skin, and respiratory irritants, with inhalation of even low levels capable of producing extreme respiratory distress $(94,155)$. Exposure to both acrolein and CR can occur naturally through foods and drinks (94), and each is produced endogenously in small amounts $(94,155-157)$. Most exposure to these compounds, however, occurs via anthropogenic sources, including vehicular exhaust, industrial emissions, and smoke from fires $(94,155-157)$, with a large portion of exposure related to tobacco smoke $(90,114$, $117,147,156,159)$. Acrolein in particular plays a significant role in mainstream cigarette smoke (MCS) and its related health effects; Stabbert et al. (149) have shown that the majority of the cytotoxicity related to the gas vapor constituents of MCS is due to acrolein, and Haussmann (145) reports that almost $90 \%$ of the non-cancer health risk (cardiovascular and pulmonary disease) associated with smoking is likely due to acrolein.

Much epidemiological and experimental evidence exists linking acrolein with increased cardiovascular disease (CVD) risk. Both acute and chronic inhalation exposures 
to acrolein have been shown to suppress levels of circulating angiogenic cells (CACs) (74, 151), enhance thrombotic markers (450), and initiate endothelium dysfunction in susceptible mice (150), while ingestion promotes atherosclerosis in mice (153). Moreover, urinary levels of 3-hydroxypropylmercapturic acid (3HPMA), the primary urinary metabolite of acrolein, are associated with increased CVD risk, decreased levels of CACs, and increased platelet-leukocyte aggregation in humans (71). By comparison, inhalation exposure to 1,3-butadiene, a parent source of $\mathrm{CR}$, accelerates arteriosclerotic lesion development in cockerels (167), and is linked with an increased risk of atherosclerosis in African-American factory workers (166). Likewise, African-American men who worked in a 1,3-butadiene production plant for at least 6 months had increases in standardized mortality ratio for arteriosclerotic heart disease (165). Furthermore, although CR has been shown to induce oxidative stress in cells $(163,164)$ and to cause cardiomyocyte dysfunction (164), CR inhalation studies with measures of cardiovascular injury have not been performed. Thus, the goal of these studies was to address these gaps in knowledge regarding the potential cardiovascular toxicity of CR.

\section{B. Experimental Procedures}

\section{Exposures}

Animals were exposed to constituents via full-body inhalation as described in Chapter II, Sections B.2. Upon completion of the exposure, mice were euthanized and tissues collected as described in Chapter II, Section B.5.

\section{Urine Collection}

Urine samples were collected post-exposure as described in Chapter II, Section B.3.

\section{Urine Metabolite Quantification}


Collected urine was used to quantify levels of volatile organic compound (VOC) and tobacco alkaloid metabolites using analytical methods as described in Chapter II, Section B.4.

\section{Complete Blood Cell Count (CBC)}

A sample of whole blood was collected after the final day of exposure for a complete blood cell count (CBC) as described in Chapter II, Section B.6.

\section{Plasma Biomarkers Quantification}

Plasma was prepared from each sample for an analysis of a number of biomarkers as described in Chapter II, Section B.7.

\section{Circulating Angiogenic Cell (CAC) Quantification}

Flow cytometry was used to analyze the number of CACs in each sample as described in Chapter II, Section B.8.

\section{Leukocyte Subpopulation Quantification}

Flow cytometry was used to analyze the leukocyte subpopulations in each sample as described in Chapter II, Section B.9.

\section{Statistical Analyses}

Statistical analyses were performed as described in Chapter II, Section B.10.

\section{Results}

1. Urinary Acrolein Metabolite in MCS-, PG:VG-, and Acrolein-Exposed Mice

The urinary metabolite of acrolein, 3HPMA, has been positively associated with increased CVD risk in smokers (71) and has been shown to be a useful biomarker of exposure to acrolein from various sources $(147,151)$. However, the use of 3HPMA as a 
biomarker of e-cig exposure has not been studied. To better understand how acrolein-alone exposure related to e-cig exposure from known ratios of PG:VG, we measured urinary 3HPMA by UPLC-MS/MS as a biomarker of acrolein exposure. For this, male mice were exposed to acrolein ( $1 \mathrm{ppm}, 6 \mathrm{~h})$, MCS (50\% of smoke of $12 \mathrm{KY}$ Reference 3R4F cigarettes, 6h), or PG:VG (50\%:50\% or 30\%:70\%, 6h), and urine was collected immediately after the exposure sessions. Acrolein alone exposure $(\mathrm{p}=0.02)$ as well as exposure to MCS $(\mathrm{p}=0.005)$ and both 50\%:50\% $(\mathrm{p}<0.001)$ and 30\%:70\% PG:VG $(\mathrm{p}=0.02)$ caused a significant increase in levels of 3HPMA in the first three hours post exposure compared with baseline (Fig. 6.1). Levels of 3HPMA in acrolein-exposed mice were similar to levels in MCS-exposed and 30\%:70\% PG:VG-exposed mice, but mice exposed to 50\%:50\% PG:VG showed significantly higher levels compared to acrolein-alone mice ( $\mathrm{p}=0.01)$ (Fig.

6.1). Comparison of 3HPMA levels in PG:VG-exposed mice showed that mice exposed to 50\%:50\% PG:VG also had a significantly higher level of 3HPMA compared with mice exposed to 30\%:70\% PG:VG (p=0.02) (Fig. 6.1).

\section{Systemic Toxicity of Acute Exposure to Inhaled Acrolein}

The unsaturated aldehyde acrolein is found in high levels in MCS $(90,114,117$, 147) and is suggested to be one of the most significant factors in MCS-induced cardiovascular toxicity $(145,149)$. Environmental exposure to acrolein has been linked to an increased CVD risk score, in part through decreased levels of select CAC populations (71), and direct inhalation exposure in mice has also caused the suppression of CACs (74, 151) as well as the related development of endothelium dysfunction (150). Chronic inhalation exposure (1 ppm) showed significant decreases in white blood cells count driven by decreases in neutrophils, lymphocytes, and monocytes (151). Because of these changes 


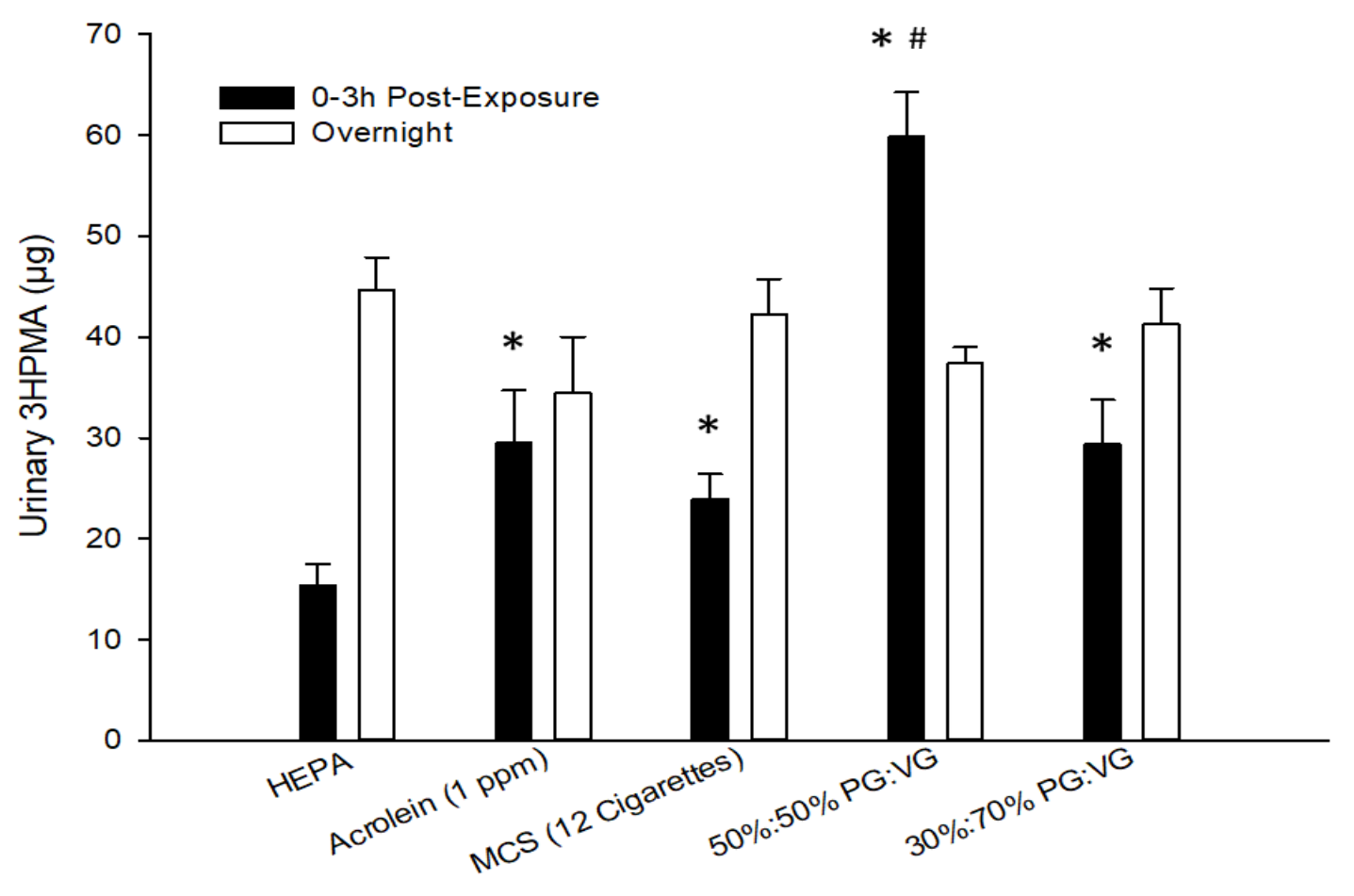

Fig. 6.1. Urinary 3HPMA levels in acrolein- or tobacco product-exposed male mice. Male mice were exposed (6h) to mainstream cigarette smoke (MCS; 50\% smoke of $12 \mathrm{KY}$ Reference 3R4F cigarettes) or propylene glycol:vegetable glycerin (PG:VG; 50\%:50\% or $30 \%: 70 \%, 6 \mathrm{~h}$ ) after which urine was collected at $0-1,1-2,2-3$, and 3-16h (overnight) and 3-hydroxypropylmercapturic acid (3HPMA) quantified. Values are mean $\pm \mathrm{SE}(\mathrm{n}=4-12$ mice per group). *, $\mathrm{p}<0.05$ compared with HEPA; \#, $\mathrm{p}<0.05$ compared with acrolein. 
noted in humans and in chronically-exposed mice, we performed an acute acrolein exposure to better understand the temporal relationship between these factors. Male mice exposed to acrolein $(1 \mathrm{ppm})$ for 4 days $(6 \mathrm{~h} / \mathrm{d})$ had a greater change in overall body weight compared with air-exposed control mice ( $-3 \mathrm{~g}$ versus $-1 \mathrm{~g}$; $\mathrm{p}=0.004)$, and the liver/body weight ratio was significantly decreased $(-10 \% ; \mathrm{p}=0.007)$ (Table 6.1). Exposure had considerable effects on CBC. Exposed mice showed a significant decrease in leukocytes $(-50 \% ; \mathrm{p}=0.02)$, driven by decreases in neutrophils $(-57 \% ; \mathrm{p}=0.02)$, lymphocytes $(-46 \%$; $\mathrm{p}=0.06)$, and monocytes $(-59 \% ; \mathrm{p}=0.03)$ (Table 6.2). Acrolein exposure also induced changes in erythrocytic parameters; red blood cell levels $(+6 \% ; \mathrm{p}=0.05)$ and hemoglobin $(+5 \% ; \mathrm{p}=0.06)$ were modestly increased, while hematocrit was significantly increased $(+6 \% ; \mathrm{p}=0.04)$ (Table 6.2). Measures of systemic biomarkers showed no changes in liver (ALT, AST), muscle (CK) or LDH enzymes or creatinine but did show a modestly significant decrease in triglycerides $(-22 \% ; \mathrm{p}=0.06)$ (Table 6.1).

\section{Acute Exposure to Acrolein and Circulating Angiogenic Cells}

Male mice acutely exposed to acrolein (1 ppm; 4d) showed no changes in the levels of CACs or Sca-1+ cells (Fig. 6.2A \& B).

\section{Urinary Crotonaldehyde Metabolite in MCS-, PG:VG-, and}

\section{Crotonaldehyde-Exposed Mice}

The primary urinary metabolite of CR, 3-hydroxy-1-methylpropylmercapturic acid (HPMMA), is associated with combusted tobacco, and the number of cigarettes per day is a significant predictor of increased urinary HPMMA (159). To better understand how CRalone exposure relates to MCS exposure from a known number of cigarettes and to e-cig exposure using a known ratio of PG:VG, we measured urinary HPMMA by UPLC-MS/MS 
Table 6.1. Systemic parameters of male C57BL/6 mice acutely exposed to either air or acrolein (1 ppm).

\begin{tabular}{|l|c|c|}
\hline \multicolumn{1}{|c|}{ Variable } & Exposure (4 days) \\
\hline Change in BWT (g) & Air Control & Acrolein (1 ppm) \\
\hline Heart/BWT (mg/g) & $-1 \pm 0$ & $-3 \pm 0^{*}$ \\
\hline Lung/BWT (mg/g) & $4.8 \pm 0.1$ & $5.0 \pm 0.1$ \\
\hline Liver/BWT (mg/g) & $5.7 \pm 0.1$ & $5.6 \pm 0.1$ \\
\hline Kidney/BWT (mg/g) Plasma Measurements & $46.0 \pm 1.1$ & $41.3 \pm 0.08^{*}$ \\
\hline \multicolumn{2}{|c|}{} & $12.6 \pm 0.2$ \\
\hline Cholesterol (mg/dL) & $12.8 \pm 0.2$ & $70.67 \pm 2.6$ \\
\hline Triglycerides (mg/dL) & $74.89 \pm 3.35$ & $43.36 \pm 3.36 \#$ \\
\hline Albumin (g/dL) & $55.33 \pm 4.31$ & $2.90 \pm 0.23$ \\
\hline Total Protein (g/dL) & $2.92 \pm 0.13$ & $4.47 \pm 0.18$ \\
\hline AST (U/I) & $3.94 \pm 0.06$ & $60.94 \pm 5.56$ \\
\hline CK (U/I) & $65.64 \pm 6.70$ & $105.87 \pm 21.03$ \\
\hline LDH (U/I) & $144.14 \pm 41.37$ & $164.29 \pm 21.52$ \\
\hline Creatinine (mg/dL) & $178.37 \pm 20.37$ & $0.23 \pm 0.02$ \\
\hline
\end{tabular}

Values = mean \pm SE (n=5-10 mice per group); Abbr.: AST, aspartate aminotransferase; BWT, body weight; CK, creatine kinase; LDH, lactate dehydrogenase; $\#, 0.05 \leq \mathrm{p} \leq 0.10$ compared with air control. 
Table 6.2. Hematological measurements of male C57BL/6 mice acutely exposed to either air or acrolein (1 ppm).

\begin{tabular}{|l|c|c|}
\hline \multicolumn{1}{|c|}{ Hematological Measurements } & Exposure (4 days) \\
\hline \multicolumn{1}{|c|}{ Acrolein (1 ppm) } \\
\hline White Blood Cell (K/uL) & $1.92 \pm 0.34$ & $0.96 \pm 0.13^{*}$ \\
\hline Neutrophils (K/uL) & $0.63 \pm 0.13$ & $0.27 \pm 0.04^{*}$ \\
\hline Lymphocytes (K/uL) & $1.24 \pm 0.23$ & $0.68 \pm 0.09 \#$ \\
\hline Monocytes (K/uL) & $0.04 \pm 0.01$ & $0.02 \pm 0.01^{*}$ \\
\hline Eosinophils (K/uL) & $0 \pm 0$ & $0 \pm 0$ \\
\hline Basophils (K/uL) & $0 \pm 0$ & $0 \pm 0$ \\
\hline Red Blood Cell (M/uL) & $8.83 \pm 0.15$ & $9.38 \pm 0.16 \#$ \\
\hline Hemoglobin (g/dL) & $12.2 \pm 0.3$ & $12.9 \pm 0.2 \#$ \\
\hline Hematocrit (\%) & $44.0 \pm 0.9$ & $46.7 \pm 0.7 *$ \\
\hline Mean Corpuscular Volume (fL) & $49.8 \pm 0.4$ & $49.8 \pm 0.3$ \\
\hline Mean Corpuscular Hemoglobin (pg) & $13.8 \pm 0.1$ & $13.8 \pm 0.1$ \\
\hline Mean Corpuscular Hemoglobin Concentration (g/dL) & $27.8 \pm 0.2$ & $27.6 \pm 0.1$ \\
\hline Red Cell Distribution Width (\%) & $17.5 \pm 0.2$ & $17.1 \pm 0.2$ \\
\hline Platelets (K/uL) & $575 \pm 27$ & $585 \pm 14$ \\
\hline Mean Platelet Volume (fL) & $4.3 \pm 0.1$ & $4.2 \pm 0.1$ \\
\hline
\end{tabular}

Values $=$ mean $\pm \mathrm{SE}(\mathrm{n}=10$ mice per group $) ; *, \mathrm{p}<0.05$ compared with air control; $\#, 0.05 \leq \mathrm{p} \leq 0.10$ compared with air control. 
A.

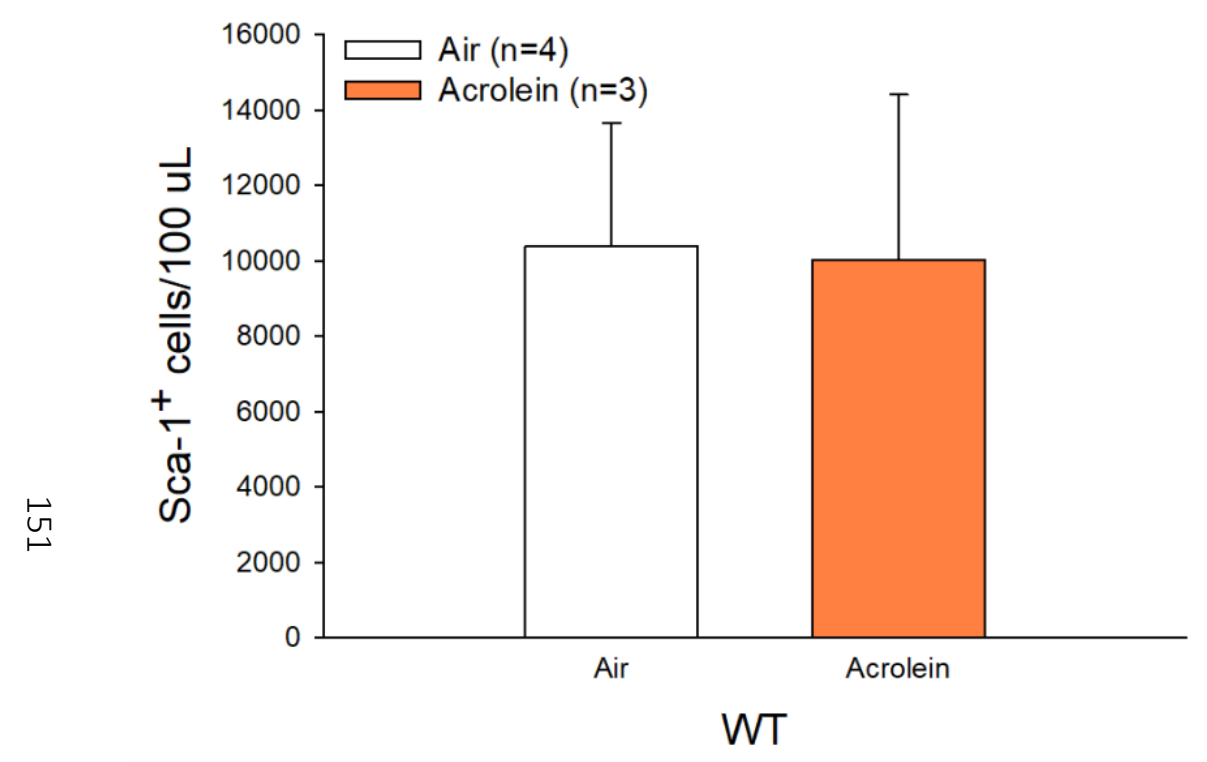

B.

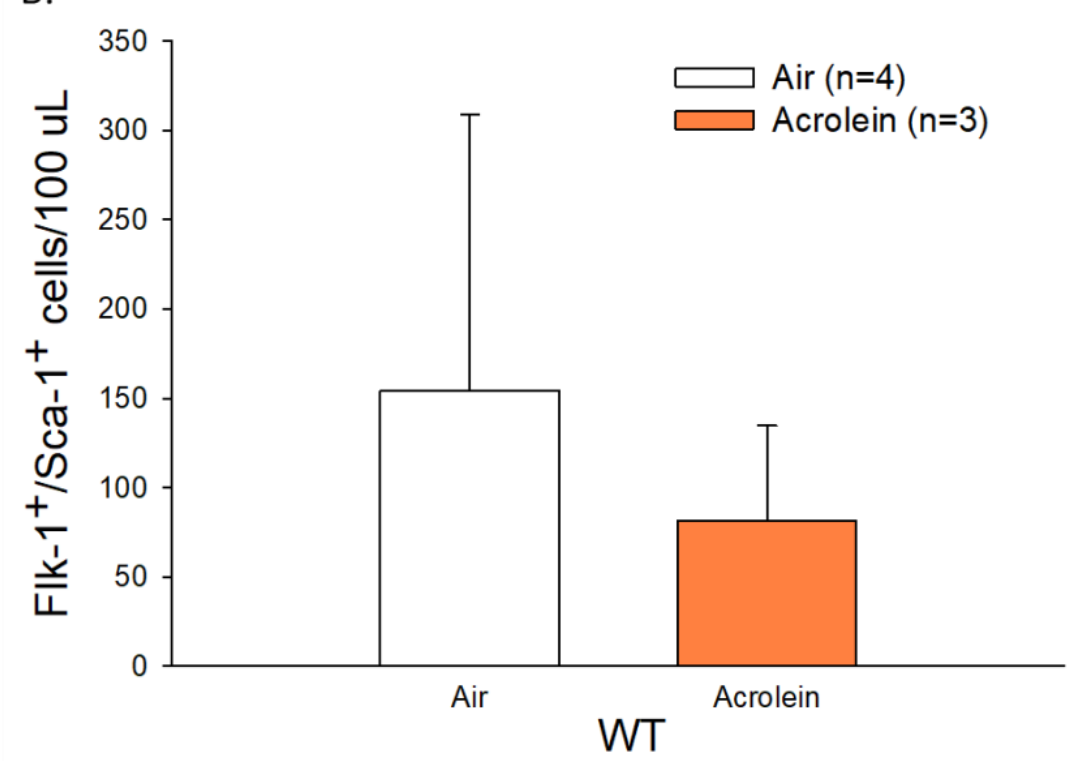

Fig. 6.2. Effects of acute acrolein (1 ppm) exposure on circulating stem cells in male mice. Hematopoietic stem cells (Sca-1+) and circulating angiogenic cells (CACs) were measured by flow cytometry in male C57BL/6J (wild type, WT) mice exposed to HEPAfiltered air or acrolein (1 ppm, 6h/d) for 4 days. Levels of circulating Sca-1+ cells (A) and CACs (Flk-1+/Sca-1+ cells; B) were unchanged in acrolein-exposed mice relative to air-exposed control mice. Values $=$ mean $\pm S E(n=3-4$ mice per group). 
as a biomarker of CR exposure (168). For this, male mice were exposed to CR (1 ppm, 6h), MCS (50\% of smoke of 12 KY Reference 3R4F cigarettes, $6 \mathrm{~h}$ ) or 50\%:50\% PG:VG (6h), and urine was collected immediately after each exposure session. Urinary HPMMA levels were significantly increased in mice exposed to CR $(\mathrm{p}<0.001)$ or MCS $(\mathrm{p}<0.001)$ compared with baseline in the first three hours post exposure, while levels in 50\%:50\% PG:VG-exposed mice remained near baseline (Fig. 6.3). Mice exposed to CR-alone also showed significantly higher levels of urinary HPMMA than mice exposed to MCS $(\mathrm{p}=0.003)$ or $50 \%: 50 \% \mathrm{PG}: \mathrm{VG}(\mathrm{p}=0.007)$ (Fig. 6.3). Unlike humans, laboratory-housed, naïve mice have modest HPMMA levels in urine, indicating minimal CR exposure via exogenous sources including ambient air or intermediary metabolism (Fig. 6.3). After 11 weeks of exposure to $1 \mathrm{ppm}$ CR exposure, the level of urinary HPMMA in the first three hours post-exposure was approximately 5-times that measured following acute MCS exposure (Fig. 6.3), indicating that MCS exposure was equivalent to $0.2 \mathrm{ppm}$ of continuous CR exposure.

\section{Systemic Toxicity of Chronic Exposure to Inhaled Crotonaldehyde}

Because chronic exposure to acrolein (12 weeks, $1 \mathrm{ppm}$ ) induced significant systemic toxicity in mice (151), we tested whether chronic CR (1 ppm) exposure would elicit similar outcomes. Body weight and organ/body weight ratios and $\mathrm{CBCs}$ were not different between the air- and CR- exposed groups (Tables $6.3 \&$ 6.4). Chronic CR also did not affect plasma levels of ALT and AST (liver), CK (striated muscle), or LDH (nonspecific cell toxicity) enzymes or creatinine, but did increase levels of plasma total cholesterol $(+11 \%$; $\mathrm{p}=0.04), \operatorname{HDL}(+16 \%$; $\mathrm{p}=0.01)$, triglycerides $(+25 \%$; $\mathrm{p}=0.01)$, albumin $(+6 \% ; \mathrm{p}=0.01)$, and, modestly, total protein $(+3 \% ; \mathrm{p}=0.08)$ compared with the air-exposed 


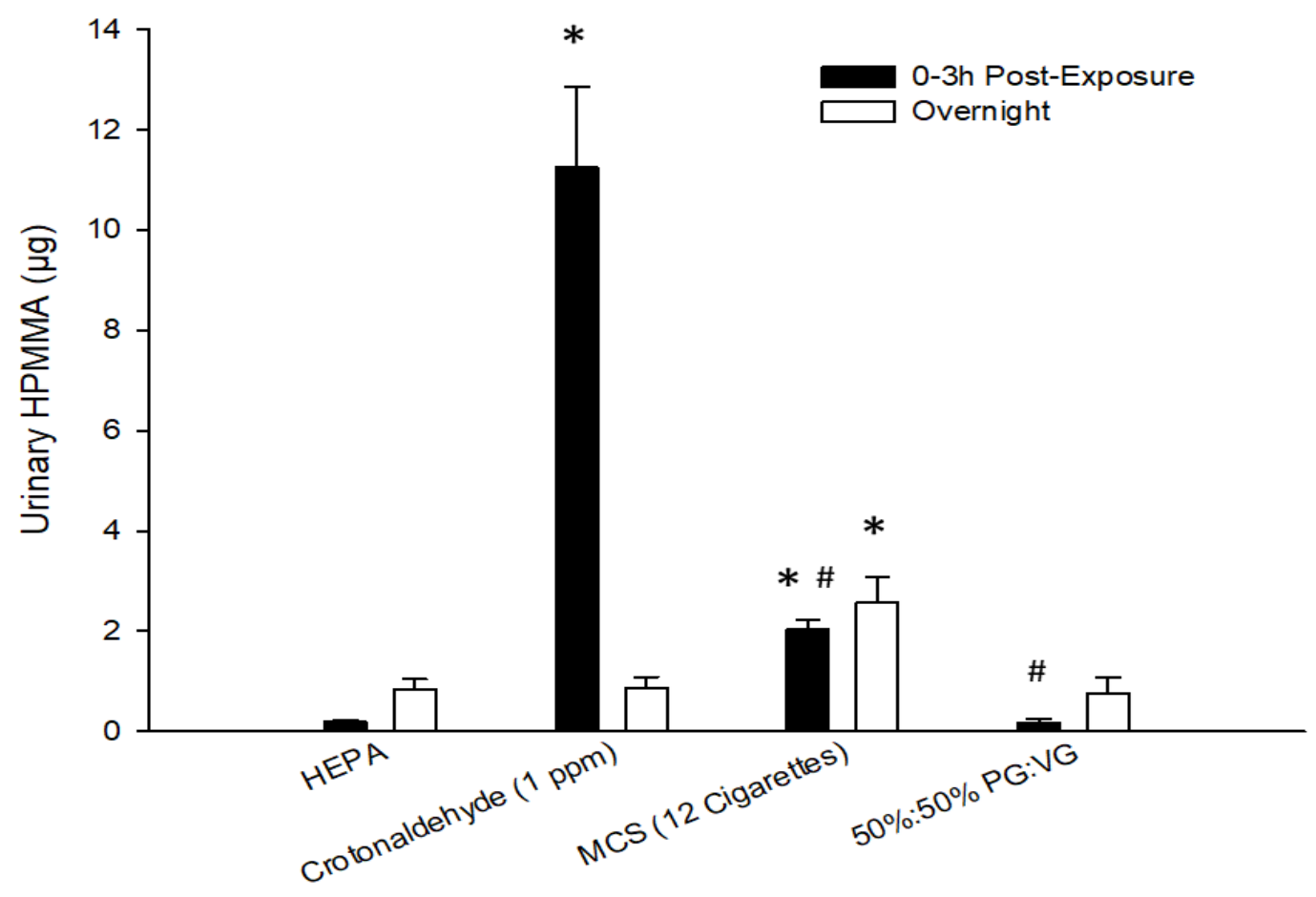

Fig. 6.3. Urinary HPMMA levels in crotonaldehyde- or tobacco product-exposed male mice. Male mice were exposed (6h) to crotonaldehyde (CR; $1 \mathrm{ppm}, 11_{\text {th }}$ week of chronic exposure), mainstream cigarette smoke (MCS; 50\% smoke of $12 \mathrm{KY}$ Reference 3R4F cigarettes), or propylene glycol:vegetable glycerin (PG:VG; 50\%:50\% or 30\%:70\%, 6h) after which urine was collected at $0-1,1-2,2-3$, and 3-16h (overnight) and 3-hydroxy1-methylpropylmercapturic acid (HPMMA) quantified. Values are mean $\pm \mathrm{SE}(\mathrm{n}=5-12$ mice per group). *, $\mathrm{p}<0.05$ compared with HEPA; \#, $\mathrm{p}<0.05$ compared with CR. 
Table 6.3. Systemic parameters in male C57BL/6J mice chronically exposed to either air or crotonaldehyde (CR; 1 ppm).

\begin{tabular}{|l|c|c|}
\hline \multicolumn{1}{|c|}{ Variable } & \multicolumn{2}{c|}{ Exposure } \\
\hline Change in BWT (g) & Air Control & CR (1 ppm) \\
\hline Heart/BWT (mg/g) & $+5 \pm 0$ & $+4 \pm 0$ \\
\hline Lung/BWT (mg/g) & $4.5 \pm 0.1$ & $4.6 \pm 0.1$ \\
\hline Liver/BWT (mg/g) & $5.4 \pm 0.2$ & $5.4 \pm 0.2$ \\
\hline Kidney/BWT (mg/g) & $44.3 \pm 1.6$ & $41.8 \pm 1.7$ \\
\hline Pancreas/BWT (mg/g) & $12.3 \pm 0.3$ & $13.1 \pm 0.5$ \\
\hline Spleen/BWT (mg/g) & $3.9 \pm 0.2$ & $4.6 \pm 0.4$ \\
\hline Epididymal Fat Pad/BWT (mg/g) & $3.1 \pm 0.2$ & $3.0 \pm 0.2$ \\
\hline \multicolumn{1}{|c|}{ Plasma Measurements } & $8.8 \pm 0.5$ & $9.0 \pm 0.8$ \\
\hline Cholesterol (mg/dL) & \multicolumn{2}{|c|}{} \\
\hline HDL (mg/dL) & $58.83 \pm 3.82$ & $69.51 \pm 2.10^{*}$ \\
\hline LDL (mg/dL) & $41.93 \pm 4.04$ & $53.05 \pm 0.94 *$ \\
\hline Triglycerides (mg/dL) & $4.94 \pm 1.01$ & $3.51 \pm 0.33$ \\
\hline Albumin (g/dL) & $37.12 \pm 2.85$ & $56.56 \pm 7.31 *$ \\
\hline Total Protein (g/dL) & $2.81 \pm 0.06$ & $2.89 \pm 0.07 *$ \\
\hline ALT (U/I) & $4.52 \pm 0.08$ & $4.67 \pm 0.08 \#$ \\
\hline AST (U/I) & $19.37 \pm 3.76$ & $15.95 \pm 1.46$ \\
\hline CK (U/I) & $49.43 \pm 13.16$ & $46.74 \pm 4.56$ \\
\hline LDH (U/I) & $260.92 \pm 39.83$ & $149.57 \pm 44.55$ \\
\hline Creatinine (mg/dL) & $83.03 \pm 8.30$ & $83.65 \pm 4.80$ \\
\hline
\end{tabular}

Values = mean \pm SE (n=9-10 mice per group). Abbr.: ALT, alanine aminotransferase; AST, aspartate aminotransferase; BWT, body weight; CK, creatine kinase; CR, crotonaldehyde; HDL, high-density lipoprotein; LDH, lactate dehydrogenase; LDL, low-density 
lipoprotein; *, $\mathrm{p}<0.05$ compared with air control based on Bonferroni’s post-test; \#, $0.05 \leq \mathrm{p} \leq 0.10$ compared with air control based on Bonferroni's post-test. 
Table 6.4. Hematological measures of male C57BL/6J mice chronically exposed to either air or crotonaldehyde (CR; 1 ppm).

\begin{tabular}{|c|c|c|}
\hline & \multicolumn{2}{|c|}{ Exposure } \\
\hline Hematological Measurements & Air Control & CR (1 ppm) \\
\hline White Blood Cell $(\mathrm{K} / \mu \mathrm{L})$ & $1.96 \pm 0.19$ & $2.12 \pm 0.18$ \\
\hline Neutrophils $(K / \mu L)$ & $0.50 \pm 0.08$ & $0.60 \pm 0.07$ \\
\hline Lymphocytes $(\mathrm{K} / \mu \mathrm{L})$ & $1.41 \pm 0.12$ & $1.47 \pm 0.14$ \\
\hline Monocytes $(\mathrm{K} / \mu \mathrm{L})$ & $0.05 \pm 0.01$ & $0.04 \pm 0.01$ \\
\hline Eosinophils $(\mathrm{K} / \mu \mathrm{L})$ & $0 \pm 0$ & $0 \pm 0$ \\
\hline Basophils $(\mathrm{K} / \mu \mathrm{L})$ & $0 \pm 0$ & $0 \pm 0$ \\
\hline Red Blood Cell $(\mathrm{M} / \mu \mathrm{L})$ & $8.30 \pm 0.15$ & $8.32 \pm 0.14$ \\
\hline Hemoglobin (g/dL) & $12.1 \pm 0.1$ & $12.0 \pm 0.1$ \\
\hline Hematocrit $(\%)$ & $35.1 \pm 0.7$ & $34.8 \pm 0.5$ \\
\hline Mean Corpuscular Volume (fL) & $42.3 \pm 0.2$ & $41.8 \pm 0.3$ \\
\hline Mean Corpuscular Hemoglobin (pg) & $14.6 \pm 0.2$ & $14.4 \pm 0.3$ \\
\hline Mean Corpuscular Hemoglobin Concentration (g/dL) & $34.6 \pm 0.5$ & $34.5 \pm 0.5$ \\
\hline Red Cell Distribution Width (\%) & $18.0 \pm 0.3$ & $18.0 \pm 0.2$ \\
\hline Platelets $(\mathrm{K} / \mu \mathrm{L})$ & $728 \pm 30$ & $694 \pm 31$ \\
\hline Mean Platelet Volume (fL) & $4.2 \pm 0.0$ & $4.3 \pm 0.1$ \\
\hline \multicolumn{3}{|l|}{ Blood Immune Cells } \\
\hline NK1.1+-Cells (count/ $\mu \mathrm{L})$ & $8.22 \pm 1.57$ & $7.00 \pm 1.11$ \\
\hline CD19+ B-Cells (count/ $\mu \mathrm{L})$ & $266.72 \pm 31.36$ & $291.24 \pm 51.23$ \\
\hline CD4+ T-Cells (count/ $\mu \mathrm{L})$ & $31.43 \pm 2.91$ & $33.79 \pm 3.10$ \\
\hline CD8+ T-Cells (count/ $\mu \mathrm{L})$ & $0.85 \pm 0.17$ & $1.04 \pm 0.18$ \\
\hline CD11b+ Monocytes $($ count $/ \mu \mathrm{L})$ & $25.01 \pm 1.95$ & $27.06 \pm 2.65$ \\
\hline Ly6Chigh Cells $($ count $/ \mu \mathrm{L})$ & $1.2 \pm 0.2$ & $1.9 \pm 0.4$ \\
\hline Ly6Clow Cells (count/ $\mu \mathrm{L})$ & $23.8 \pm 1.9$ & $25.2 \pm 2.5$ \\
\hline Gr1+ Granulocytes $($ count $/ \mu \mathrm{L})$ & $69.87 \pm 5.21$ & $82.52 \pm 7.51$ \\
\hline
\end{tabular}

Values $=$ mean \pm SE (n=9-10 mice per group). Abbr.: $\mathbf{C R}$, crotonaldehyde. 
control group (Table 6.3).

\section{Chronic Exposure to Crotonaldehyde and Hematology}

Chronic exposures to acrolein (12 weeks, 0.5 and $1 \mathrm{ppm})$ suppress a number of leukocyte subpopulations (151). Thus, in an attempt to understand any selective hematological effects of $\mathrm{CR}$, we used hematological measures to quantify changes in circulating leukocytes and selective immune subpopulations of mice chronically exposed to $\mathrm{CR}$. CR-exposed mice had no changes in major leukocyte classes, and there were no significant changes in levels of NK1.1+-cells, CD19+ B-cells, CD4+ or CD8+ T-cells, Gr1+ granulocytes, CD11b+ monocytes, or Ly6Chigh or Ly6Clow monocyte sub-populations (Table 6.4).

\section{Chronic Exposure to Crotonaldehyde and Circulating Angiogenic Cells}

Decreased levels of CACs have been associated with increased CVD risk, and it is thought that CAC levels serve as a predictor of overall cardiovascular health and future

cardiovascular events (66). Altered CAC levels are noted in individuals exposed to secondhand smoke (72) and during smoking cessation (318). Previously, we have shown that both (74) acute and chronic (151) exposures of mice to $1 \mathrm{ppm}$ acrolein suppressed CACs by $50 \%$. Despite the changes seen with acrolein, there was no significant change in the level of Sca-1+ or CACs (Flk-1+/Sca-1+ cells) after chronic CR exposure (Fig. 6.4A \& B).

\section{Systemic Toxicity of Acute Exposure to Inhaled Crotonaldehyde}

Although chronic exposure to CR (1 ppm) had specific yet limited systemic toxicity, we performed acute CR exposures to better understand the temporal relationships between these factors. Thus, we tested whether acute CR exposures would induce rapid 
A.

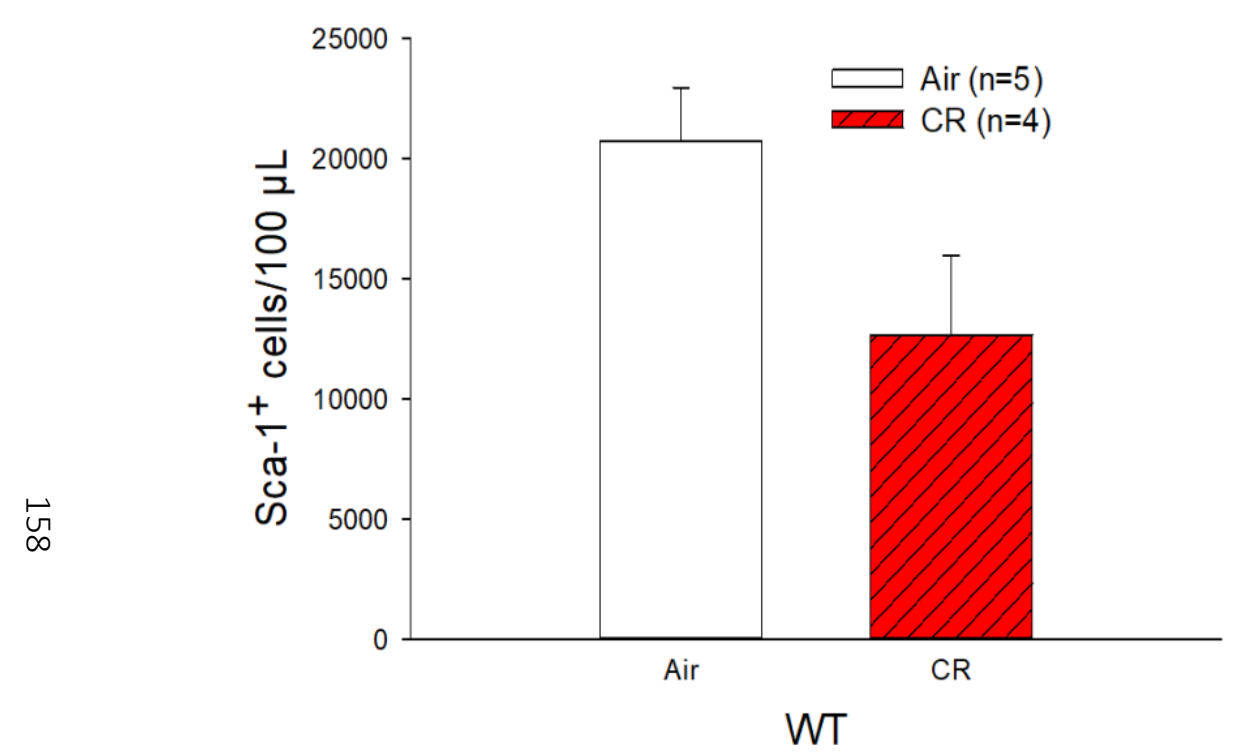

B.

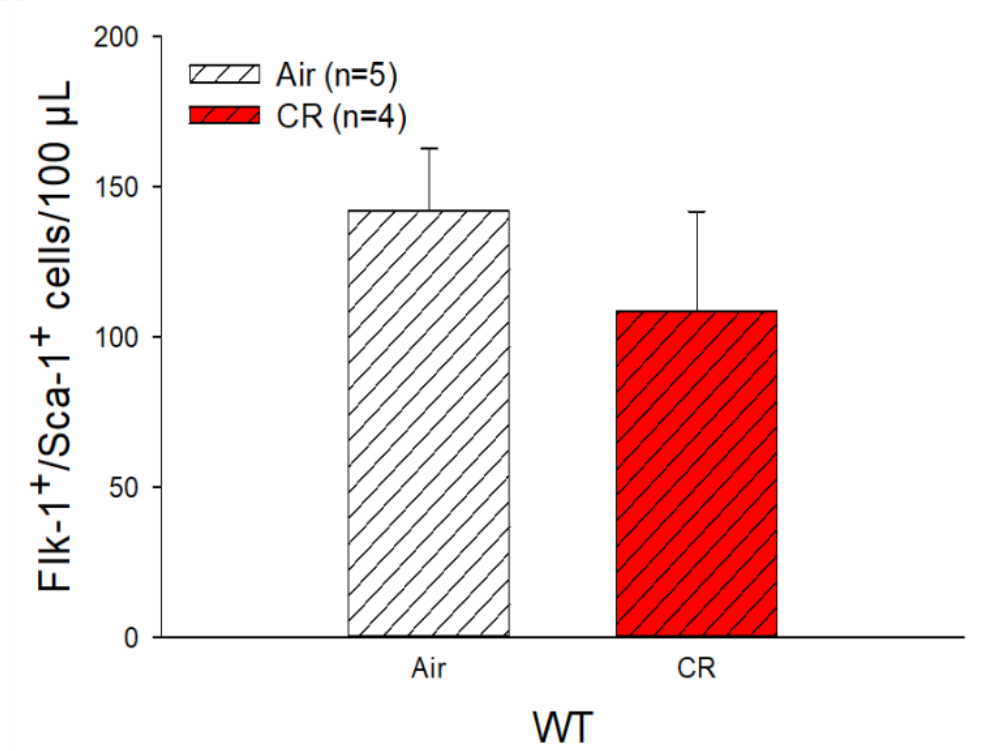

Fig. 6.4. Effects of chronic crotonaldehyde (CR; 1 ppm) exposure on circulating stem cells in male mice. Hematopoietic stem cells (Sca-1+) and circulating angiogenic cells (CACs) were measured by flow cytometry in male C57BL/6J (wild type, WT) mice exposed to HEPA-filtered air or CR (1 ppm, 6h/d) for 12 weeks. Levels of circulating Sca-1+cells (A) and CACs (Flk-1+/Sca-1+cells; B) were unchanged in CR-exposed mice relative to air control mice. Values $=$ mean $\pm \mathrm{SE}$ ( $\mathrm{n}=4-5$ mice per group). 
toxicity that was otherwise compensated for in the chronic setting. Acute exposure of male mice to CR (1 or $3 \mathrm{ppm} ; 4$ days) had limited to modest effects on organ and body weights (or ratios), $\mathrm{CBC}$, and plasma biomarkers. Male mice acutely exposed to $1 \mathrm{ppm} \mathrm{CR}$ had no change in overall body weight, yet had an increased level of neutrophils $(+60 \% ; \mathrm{p}=0.06)$, a decreased mean corpuscular volume $(-6 \%$; $\mathrm{p}<0.001)$, and an increased red blood cell distribution width (+6\%; $\mathrm{p}=0.02$ ) (Table 6.5). Plasma levels of ALT, ASK, CK, and LDH enzymes, albumin, and creatinine were unchanged with acute CR exposure in WT male mice, however, levels of plasma cholesterol, LDL, triglycerides and total protein were decreased $(-14 \%, \mathrm{p}=0.002 ;-6 \%, \mathrm{p}=0.02 ;-13 \%, \mathrm{p}=0.02 ;-24 \%, \mathrm{p}=0.002$, respectively $)$ (Table 6.6). Surprisingly, 4 days of CR (3 ppm) exposure in male mice had less of an effect overall on markers of systemic toxicity. For example, there were no changes in levels of ALT, AST, CK, and LDH enzymes, albumin, or creatinine or any changes in hematological biomarkers, with only modest effects on levels of cholesterol $(+7 \%$; $=0.09)$ and total protein $(+5 \%$; $\mathrm{p}=0.07)$ (Tables 6.6$)$. Similarly, no changes in body weight, organ/body weight ratios, $\mathrm{CBC}$, or plasma biomarkers were observed in female mice exposed for 4 days to $1 \mathrm{ppm} \mathrm{CR}$ (Table 6.7), indicating that male WT mice may be more sensitive to CR than their female counterparts.

\section{Acute Exposure to Crotonaldehyde and Circulating Angiogenic Cells}

Acute (4d) exposure to $1 \mathrm{ppm} \mathrm{CR}$ increased the level of Sca-1+ cells (Fig. 6.5A); Sca-1+ cells make up the hematopoietic stem cell pool from which CACs are derived. However, there was no change in the levels of CACs (Fig. 6.5B). Acute (4d) exposure to 3 ppm CR did not change the levels of Sca-1+ cells or CACs (Fig. 6.6A \& B). Likewise, female WT mice had no change in the levels of CACs or Sca-1+ cells with acute (4d) CR 
Table 6.5. Systemic measures in male C57BL/6J mice acutely exposed to either air or crotonaldehyde (CR; 1 or 3 ppm).

\begin{tabular}{|c|c|c|c|}
\hline \multirow[b]{2}{*}{ Variable } & \multicolumn{3}{|c|}{ Exposure Duration (4 days) } \\
\hline & Air Control & CR (1 ppm) & CR (3 ppm) \\
\hline Change in BWT (g) & $-1 \pm 0$ & $-1 \pm 0$ & $-1 \pm 0$ \\
\hline Heart/BWT (mg/g) & $4.9 \pm 0.1$ & $5.2 \pm 0.1$ & $4.9 \pm 0.1$ \\
\hline Lung/BWT (mg/g) & $5.6 \pm 0.1$ & $6.0 \pm 0.1$ & $5.4 \pm 0.1$ \\
\hline Liver/BWT (mg/g) & $44.6 \pm 0.6$ & $43.7 \pm 1.5$ & $43.6 \pm 0.9$ \\
\hline Kidney/BWT (mg/g) & $12.5 \pm 0.1$ & $12.7 \pm 0.3$ & $12.4 \pm 0.2$ \\
\hline \multicolumn{4}{|l|}{ Hematological Measurements } \\
\hline White Blood Cell $(\mathrm{K} / \mu \mathrm{L})$ & $1.57 \pm 0.13$ & $1.96 \pm 0.16$ & $1.55 \pm 0.14$ \\
\hline Neutrophils $(K / \mu \mathrm{L})$ & $0.39 \pm 0.06$ & $0.62 \pm 0.10 \#$ & $0.29 \pm 0.03$ \\
\hline Lymphocytes $(\mathrm{K} / \mu \mathrm{L})$ & $1.13 \pm 0.09$ & $1.28 \pm 0.10$ & $1.22 \pm 0.11$ \\
\hline Monocytes $(\mathrm{K} / \mu \mathrm{L})$ & $0.04 \pm 0$ & $0.06 \pm 0.01$ & $0.04 \pm 0$ \\
\hline Eosinophils $(\mathrm{K} / \mu \mathrm{L})$ & $0.01 \pm 0$ & $0 \pm 0$ & $0 \pm 0$ \\
\hline Basophils $(\mathrm{K} / \mu \mathrm{L})$ & $0 \pm 0$ & $0 \pm 0$ & $0 \pm 0$ \\
\hline Red Blood Cell $(\mathrm{M} / \mu \mathrm{L})$ & $8.62 \pm 0.10$ & $8.71 \pm 0.10$ & $8.83 \pm 0.11$ \\
\hline Hemoglobin (g/dL) & $11.7 \pm 0.2$ & $11.7 \pm 0.2$ & $11.9 \pm 0.1$ \\
\hline Hematocrit (\%) & $39.2 \pm 0.8$ & $37.5 \pm 0.7$ & $42.1 \pm 0.5$ \\
\hline Mean Corpuscular Volume (fL) & $45.9 \pm 0.5$ & $43.1 \pm 0.5^{*}$ & $47.7 \pm 0.3$ \\
\hline Mean Corpuscular Hemoglobin (pg) & $13.6 \pm 0.2$ & $13.5 \pm 0.3$ & $13.5 \pm 0.1$ \\
\hline Mean Corpuscular Hemoglobin Concentration (g/dL) & $29.6 \pm 0.6$ & $31.4 \pm 1.1$ & $28.3 \pm 0.1$ \\
\hline Red Cell Distribution Width (\%) & $16.9 \pm 0.1$ & $17.9 \pm 0.3^{*}$ & $17.2 \pm 0.3$ \\
\hline Platelets $(\mathrm{K} / \mu \mathrm{L})$ & $616 \pm 16$ & $670 \pm 25$ & $584 \pm 15$ \\
\hline Mean Platelet Volume (fL) & $4.3 \pm 0$ & $4.2 \pm 0.1$ & $4.3 \pm 0$ \\
\hline
\end{tabular}

Values $=$ mean \pm SE (n=9-15 mice per group); Abbr.: BWT, body weight; $\mathbf{C R}$, crotonaldehyde; $*$, p $<0.05$ compared with air control based on Bonferroni's or Dunn's post-test; $0.05 \leq \mathrm{p} \leq 0.10$ compared with air control based on Bonferroni's or Dunn's post-test. 
Table 6.6. Plasma biomarkers in male C57BL/6J mice acutely exposed to either air or crotonaldehyde (CR; 1 or 3 ppm).

\begin{tabular}{|c|c|c|c|}
\hline & \multicolumn{3}{|c|}{ Exposure Duration (4 days) } \\
\hline Plasma Measurements & Air Control & CR (1 ppm) & CR (3 ppm) \\
\hline Cholesterol (mg/dL) & $69.97 \pm 1.83$ & $60.16 \pm 1.84 *$ & $74.72 \pm 1.89_{\#}$ \\
\hline HDL (mg/dL) & $50.96 \pm 1.21$ & $44.57 \pm 2.31^{*}$ & $53.10 \pm 1.43$ \\
\hline LDL (mg/dL) & $4.49 \pm 0.37$ & $4.32 \pm 0.62$ & $5.52 \pm 0.19$ \\
\hline Triglycerides (mg/dL) & $43.76 \pm 1.39$ & $33.36 \pm 2.39 *$ & $41.31 \pm 2.22$ \\
\hline Albumin $(g / d L)$ & $2.81 \pm 0.04$ & $2.65 \pm 0.05$ & $2.85 \pm 0.05$ \\
\hline Total Protein (g/dL) & $4.76 \pm 0.07$ & $4.45 \pm 0.06^{*}$ & $5.01 \pm 0.08 \#$ \\
\hline ALT (U/I) & $23.34 \pm 1.25$ & $23.74 \pm 2.92$ & $19.37 \pm 0.63$ \\
\hline AST (U/I) & $61.99 \pm 4.97$ & $56.39 \pm 6.12$ & $52.86 \pm 4.05$ \\
\hline CK (U/I) & $188.84 \pm 21.28$ & $127.15 \pm 13.94$ & $262.70 \pm 33.38$ \\
\hline LDH (U/I) & $135.90 \pm 7.72$ & $132.28 \pm 11.21$ & $141.10 \pm 10.36$ \\
\hline Creatinine (mg/dL) & $0.28 \pm 0.01$ & $0.24 \pm 0.01 \#$ & $0.31 \pm 0.01$ \\
\hline
\end{tabular}

Values = mean \pm SE (n=5-10 mice per group); Abbr.: ALT, alanine aminotransferase; AST, aspartate aminotransferase; CK, creatine kinase; CR, crotonaldehyde; HDL, high-density lipoprotein; LDH, lactate dehydrogenase; LDL, low-density lipoprotein; *, p<0.05 compared with air control based on Bonferroni's or Dunn's post-test; \#, $0.05 \leq \mathrm{p} \leq 0.10$ compared with air control based on Bonferroni's or Dunn's post-test; $\uparrow, \mathrm{p}<0.05$ for WT vs TRPA1-null based on Dunn's post-test. 
Table 6.7. Systemic measures in female C57BL/6J mice acutely exposed to either air or crotonaldehyde (CR; 1 ppm).

\begin{tabular}{|c|c|c|}
\hline \multirow[b]{2}{*}{ Variable } & \multicolumn{2}{|c|}{ Exposure } \\
\hline & Air Control & CR (1 ppm) \\
\hline Change in BWT (g) & $-1 \pm 0$ & $-1 \pm 0$ \\
\hline Heart/BWT (mg/g) & $5.2 \pm 0.1$ & $5.0 \pm 0.1$ \\
\hline Lung/BWT (mg/g) & $6.6 \pm 0.1$ & $6.3 \pm 0.2$ \\
\hline Liver/BWT (mg/g) & $45.4 \pm 1.8$ & $44.4 \pm 1.4$ \\
\hline Kidney/BWT (mg/g) & $13.3 \pm 0.4$ & $13.1 \pm 0.2$ \\
\hline \multicolumn{3}{|c|}{ Hematological Measurements $(\mathrm{n}=9-10$ mice per group) } \\
\hline White Blood Cell $(\mathrm{K} / \mu \mathrm{L})$ & $1.30 \pm 0.14$ & $1.15 \pm 0.16$ \\
\hline Neutrophils $(\mathrm{K} / \mu \mathrm{L})$ & $0.30 \pm 0.05$ & $0.26 \pm 0.05$ \\
\hline Lymphocytes $(\mathrm{K} / \mu \mathrm{L})$ & $0.96 \pm 0.10$ & $0.86 \pm 0.12$ \\
\hline Monocytes $(\mathrm{K} / \mu \mathrm{L})$ & $0.03 \pm 0$ & $0.03 \pm 0.01$ \\
\hline Eosinophils $(\mathrm{K} / \mu \mathrm{L})$ & $0 \pm 0$ & $0 \pm 0$ \\
\hline Basophils $(\mathrm{K} / \mu \mathrm{L})$ & $0 \pm 0$ & $0 \pm 0$ \\
\hline Red Blood Cell count $(\mathrm{M} / \mu \mathrm{L})$ & $8.99 \pm 0.10$ & $9.05 \pm 0.13$ \\
\hline Hemoglobin (g/dL) & $13.0 \pm 0.2$ & $13.3 \pm 0.1$ \\
\hline Hematocrit (\%) & $44.4 \pm 0.5$ & $44.9 \pm 0.7$ \\
\hline Mean Corpuscular Volume (fL) & $49.4 \pm 0.4$ & $49.6 \pm 0.3$ \\
\hline Mean Corpuscular Hemoglobin (pg) & $14.4 \pm 0.2$ & $14.7 \pm 0.3$ \\
\hline Mean Corpuscular Hemoglobin Concentration & $29.2 \pm 0.5$ & $29.7 \pm 0.5$ \\
\hline Red Cell Distribution Width (\%) & $16.5 \pm 0.2$ & $16.6 \pm 0.2$ \\
\hline Platelets $(\mathrm{K} / \mu \mathrm{L})$ & $499 \pm 16$ & $503 \pm 10$ \\
\hline Mean Platelet Volume (fL) & $4.3 \pm 0$ & $4.2 \pm 0.1$ \\
\hline \multicolumn{3}{|c|}{ Plasma Measurements $(\mathrm{n}=7-8$ mice per group) } \\
\hline Cholesterol (mg/dL) & $57.40 \pm 4.68$ & $54.87 \pm 2.66$ \\
\hline HDL (mg/dL) & $34.37 \pm 1.67$ & $35.34 \pm 2.55$ \\
\hline LDL (mg/dL) & $6.70 \pm 0.45$ & $6.27 \pm 0.40$ \\
\hline
\end{tabular}




\begin{tabular}{|l|c|c|}
\hline Albumin $(\mathbf{g} / \mathbf{d L})$ & $2.91 \pm 0.03$ & $2.92 \pm 0.06$ \\
\hline Total Protein $\mathbf{( g / d L )}$ & $4.54 \pm 0.09$ & $4.56 \pm 0.09$ \\
\hline
\end{tabular}

Values = mean \pm SE. Abbr.: BWT, body weight; CR, crotonaldehyde; HDL, high-density lipoprotein; LDL, low-density lipoprotein. 
A.
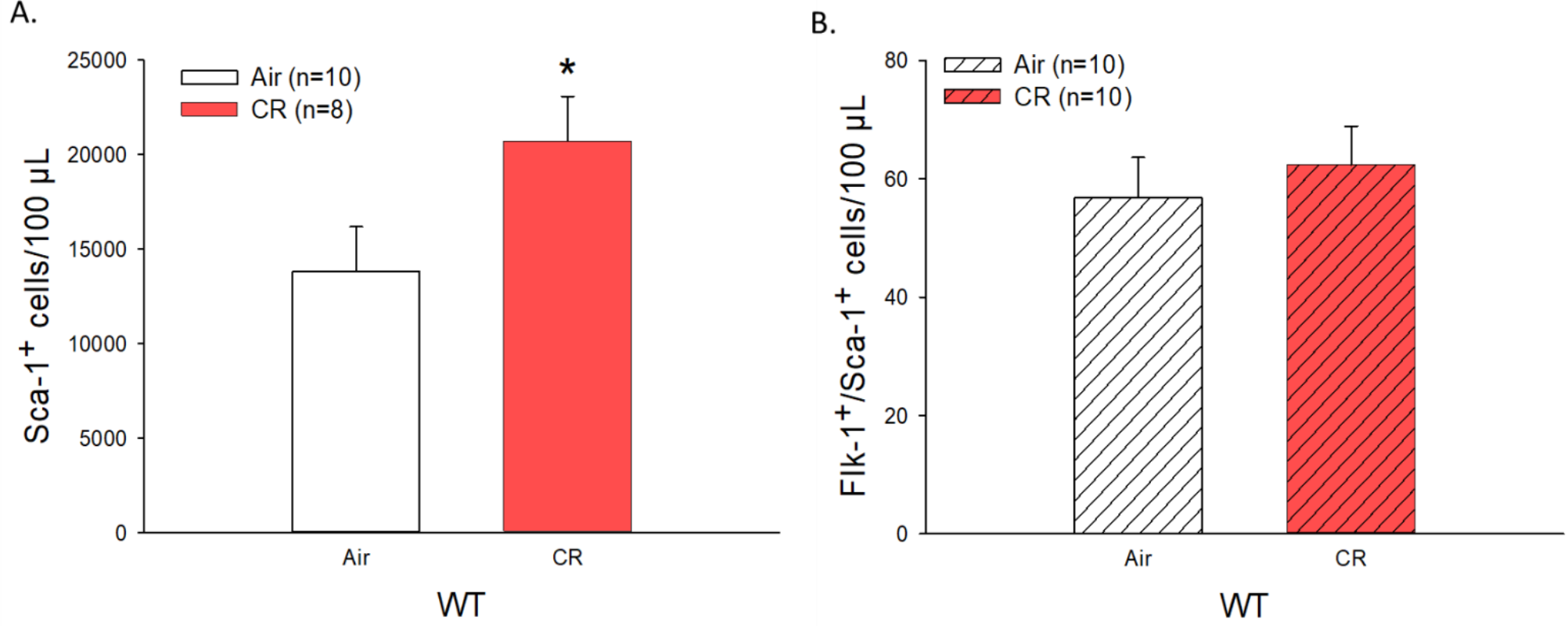

Fig. 6.5. Effects of acute crotonaldehyde $(\mathrm{CR} ; 1 \mathrm{ppm})$ exposure on circulating stem cells in male mice. Hematopoietic stem cells (Sca-1+) and circulating angiogenic cells (CACs) were measured by flow cytometry in male C57BL/6J (wild type, WT) mice exposed to HEPA-filtered air or CR (1 ppm, 6h/d) for 4 days. After 4 days of exposure to CR, the level of circulating Sca-1+ cells was significantly increased in WT mice (A), however, no change in the level of CACs (Flk-1+/Sca-1+ cells) was detected relative to airexposed control mice $(\mathbf{B})$. Values $=$ mean $\pm \mathrm{SE}(\mathrm{n}=10$ mice per group);, $\mathrm{p}<0.05$ compared with air control based on Bonferroni's or Dunn's post-test. 
A.

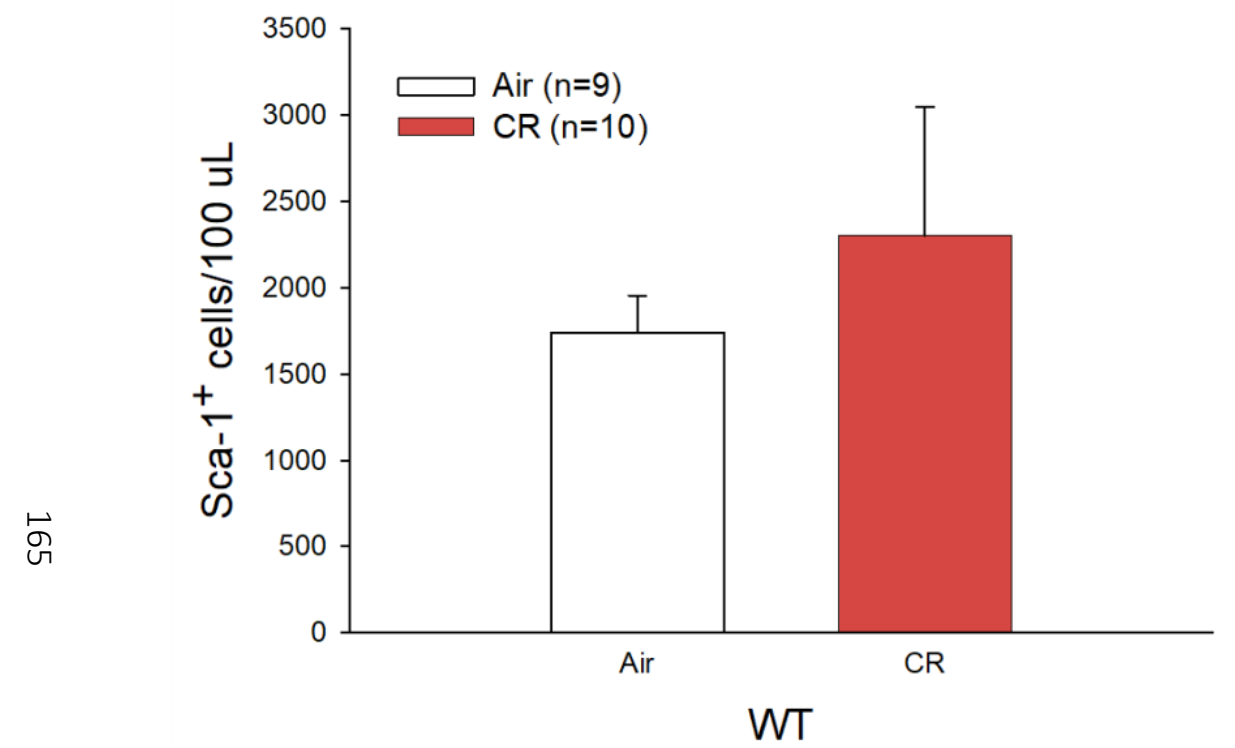

B.

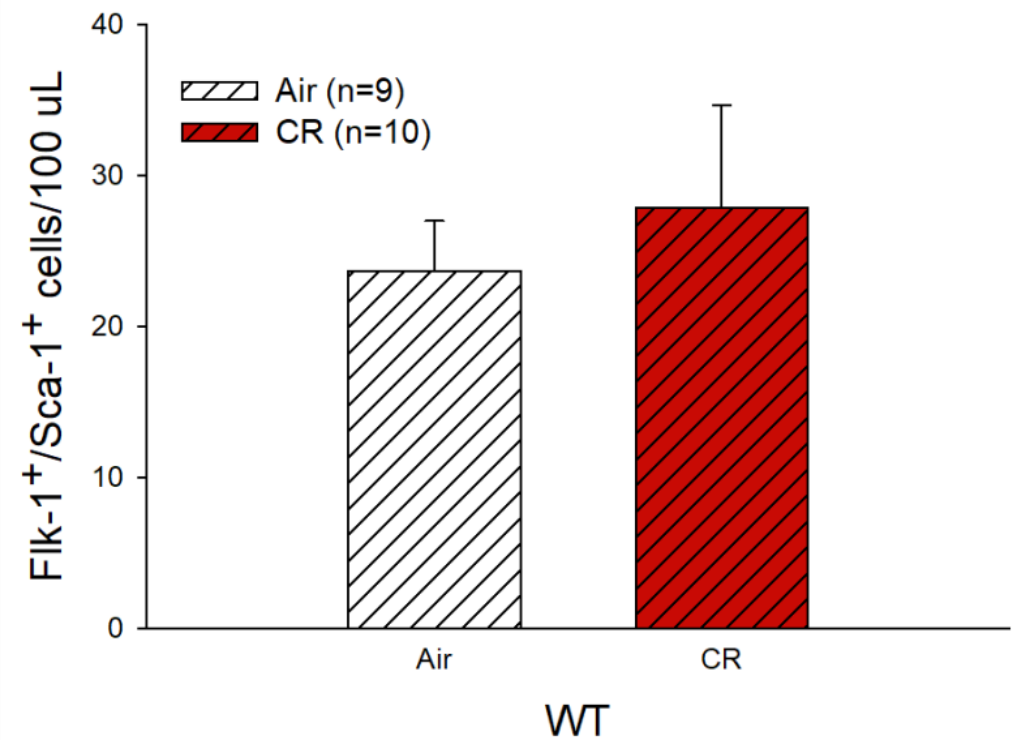

Fig. 6.6. Effects of acute crotonaldehyde (CR; 3 ppm) exposure on circulating stem cells in male mice. Hematopoietic stem cells (Sca-1+) and circulating angiogenic cells (CACs) were measured by flow cytometry in male C57BL/6J (wild type, WT) mice exposed to HEPA-filtered air or CR (1 ppm, 6h/d) for 4 days. Levels of circulating Sca-1+ cells (A) and CACs (Flk-1+/Sca-1+ cells; B) were unchanged in CR-exposed mice relative to air control mice. Values $=$ mean $\pm \mathrm{SE}(\mathrm{n}=9-10$ mice per group). 
(1 ppm) exposure (Fig. 6.7A \& B).

\section{Discussion}

MCS is a known source of the unsaturated aldehydes acrolein (18-98 $\mu \mathrm{g} /$ cigarette) and CR (10-43 $\mu \mathrm{g} / \mathrm{cigarette})(147,451)$, and levels of the major urinary metabolites of acrolein (3HPMA) and CR (HPMMA) are significantly increased in smokers versus nonsmokers $(147,159)$. The results of our urinary metabolite studies showed significant increases in both 3HPMA and HPMMA compared with baseline measures. Exposure to MCS and 30\%:70\% PG:VG produced similar levels of 3HPMA, while 50\%:50\% PG:VG produced a peak level higher than even that measured after acrolein-alone exposure (151). Conklin et al. (168) showed that levels of acrolein detected in PG-alone aerosol were below the detectable limit, while VG-alone produced levels of acrolein comparable to those measured in flavored e-liquids. Our results, however, indicate that PG is likely driving the production of acrolein, again suggesting that perhaps PG and VG in combination are capable of producing levels of aldehydes that vary compared with PG or VG alone. Regardless, it seems clear that the proportion of PG:VG used in an e-cig device can create significant variation in the aldehydes produced. Conversely, while levels of HPMMA were not below the limit of detection in 50\%:50\% PG:VG-exposed mice, they were very low and not significantly different from baseline. Previous studies have reported little if any generation of CR in e-cigs $(96,168)$, so HPMMA should not be used as a biomarker of ecig use. HPMMA is, however, increased after exposure to MCS, meaning HPMMA would be a more selective biomarker for MCS exposure. Chronic exposure to CR-alone showed a rapid increase in urinary HPMMA, with the level after 11 weeks of exposure exceeding that of MCS more than 5-fold within the first three hours post-exposure. This supports 
A.

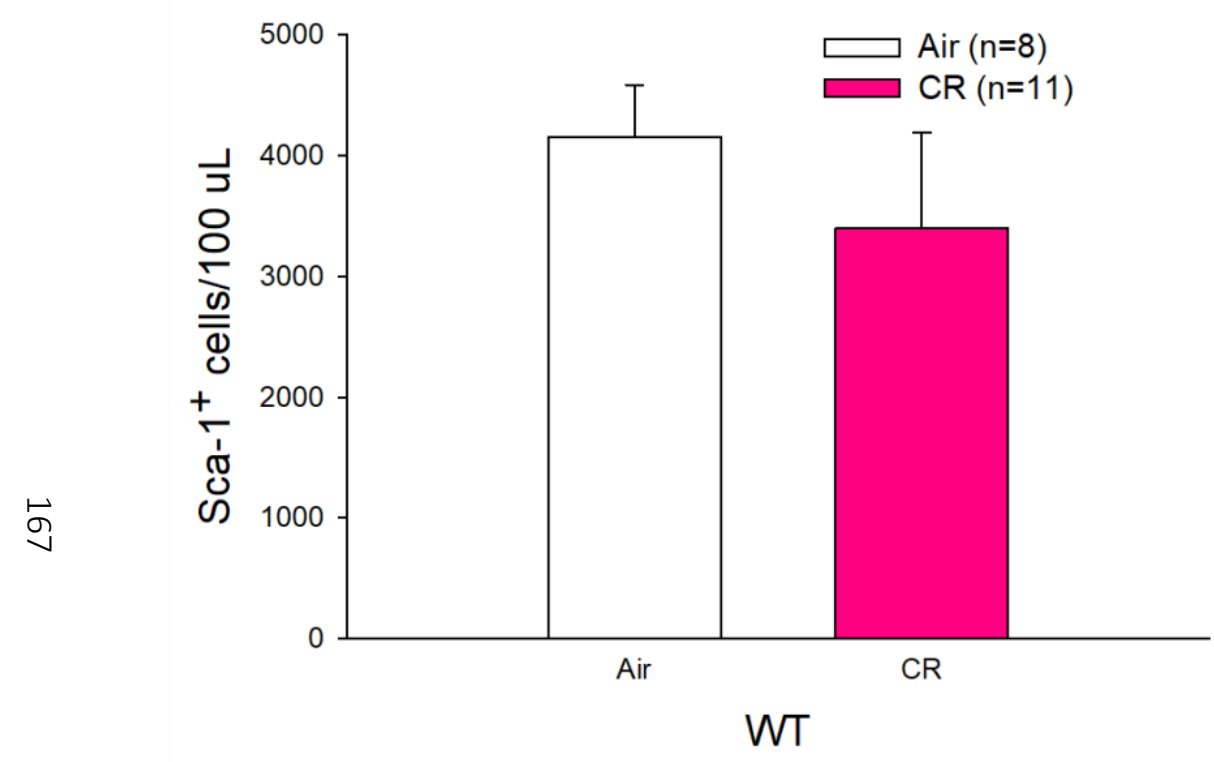

B.

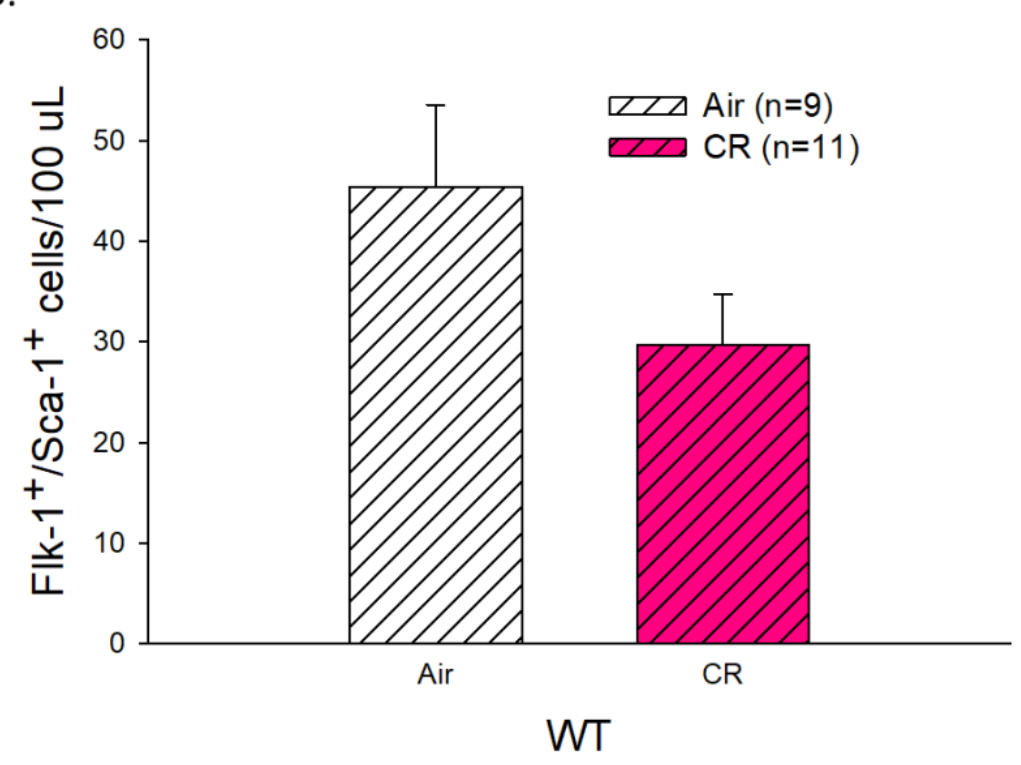

Fig. 6.7. Effects of acute crotonaldehyde (CR; 1 ppm) exposure on circulating stem cells in female mice. Hematopoietic stem cells (Sca-1+) and circulating angiogenic cells (CAC) were measured by flow cytometry gating in female C57BL/6J (wild type, WT) mice exposed to HEPA-filtered air or CR (1 ppm, 6h/d) for 4 days. Levels of circulating Sca-1+ cells (A) and CACs (Flk-1+/Sca-1+ cells; B) were unchanged in CR-exposed mice relative to air control mice. Values = mean $\pm \mathrm{SE}$ ( $\mathrm{n}=8-11$ mice per group). 
previous findings that levels of CR in MCS are not as high as levels of acrolein but that there is a significant level of CR in cigarettes that may contribute to the cardiovascular effects of smoking.

A number of epidemiological and experimental studies have linked acrolein exposure with increased CVD risk. Previous studies in our group have shown that acrolein contributes to the development of CVD through CAC suppression $(74,151)$, platelet activation (450), and endothelium dysfunction (150). However, acute (4d; 1 ppm) exposure to acrolein produced no systemic toxicity as measured by $\mathrm{CBC}$ or measured in plasma biomarkers (74), and even chronic exposure (12 weeks; 1 ppm) only showed significant changes in leukocytes (151). The current acute (4d) study shows significant decreases in leukocytes, but only minimal changes in plasma biomarkers, and while we did not see a significant decrease in levels of CACs as was previously seen after acrolein exposure $(74,151)$, there was a trend towards decreased levels that likely did not reach significance due to our small sample size ( $n=4$ air; $n=3$ acrolein). Although the change in leukocytes seen in the current acute study (1 ppm) vary from the results of the previous study (74), they support the findings of the chronic study (151), indicating that the variation between the acute studies may simply be due to the use of a different set of mice. However, the significant decrease (74) and trending decrease in CACs in the acute studies likely indicate that acrolein primarily induces injury in the endothelium before inducing more systemic changes, although these changes may still occur at a time point earlier than 12 weeks.

Despite the physical and chemical similarities and the shared exposure sources between acrolein and $\mathrm{CR}$ in addition to the well-recognized toxicity of $\mathrm{CR}$, the chronic 
toxicity of inhaled CR is not well studied. To our knowledge, these studies are the first to evaluate the systemic and vascular effects of acute and chronic CR inhalation exposure. Although CR is known to trigger inflammation (91), the current data do not show changes in circulating leukocytes or specific immune cell differentials in either acute (1 or 3 ppm) or chronic exposures, an outcome differing from that after acute (4d, $1 \mathrm{ppm})$ and chronic acrolein exposure (12 weeks; $1 \mathrm{ppm})(74,151)$. Of course, CR exposure could lead to the suppression of immune function $(452,453)$ independent of leukocyte numbers as described for alveolar macrophages following acrolein exposure (454). However, these other studies of CR or acrolein are hard to compare with the current studies due to the high-level CR doses given by intratracheal installation that led to mortality (452) or to the use of in vitro methods $(453,454)$. From the inhalation studies presented here, it can be inferred that the immune system overall appears a less sensitive target of CR exposure than of acrolein.

In other systemic measures, chronic CR exposure results in dyslipidemic changes through increases in both cholesterol and triglycerides. High levels of cholesterol and triglycerides are known risk factors of ischemic (455) and coronary heart disease (456). Triglycerides release pre-formed mediators of oxidative stress that influence endothelial cell function by stimulating intracellular production of reactive oxygen species (457), inducing endothelium dysfunction $(458,459)$, and promoting atherosclerotic plaques (460). Although levels of both cholesterol and triglycerides increased in this chronic study, neither biomarker increased after acute (4d; $1 \mathrm{ppm})$ exposure. In fact, both cholesterol and triglycerides significantly decrease in the CR-exposed group compared with air-exposed control mice, indicating that chronic increases in lipids are not the primary change initiated by CR exposure. 
Over the past decade, studies in our group have shown that exposures of mice and humans to air pollutants (particulate matter (PM2.5), acrolein) lead to the suppression of CACs $(71,73,74)$, yet no changes in CACs were observed in these studies. It can therefore be inferred that $1 \mathrm{ppm} \mathrm{CR}$ is not as potent of a stimulus of CAC suppression as is either PM2.5 or acrolein (1 ppm).

These studies also examined the effect of sex on the measured CR-induced outcomes. Female mice acutely exposed to CR (4d; 1 ppm) showed no significant changes in any systemic or vascular biomarkers, indicating that the effects of CR exposure are likely sex-dependent. Epidemiological studies seem to support this outcome; analysis of male versus female smokers in the National Health and Nutrition Examination Study (NHANES) 2005-2006 and 2011-2012 cohorts showed significant differences in urinary HPMMA (159). As previous studies in acrolein-exposed mice also show that the effects of acrolein are sex-dependent (269), it may be that there are sex differences in the pharmacodynamics and pharmacokinetics in the metabolism of the unsaturated aldehydes. Additional studies of acrolein and CR exposure in both male and female mice should work to identify the mechanism of this female-dependent protection with the idea that this clarification could be used in the development of effective interventions and treatments for unsaturated aldehyde exposure.

In summary, the studies presented in this chapter overall confirm the results of previous work examining the effects of acrolein exposure and have demonstrated the systemic and hematological effects of both acute and chronic CR exposure. In particular, chronic CR exposure was shown to increase levels of plasma biomarkers indicative of dyslipidemia and possibly subsequent promotion of atherosclerotic development. 
Comparisons of the outcomes between acrolein and CR seem to demonstrate that acrolein is more toxic than $\mathrm{CR}$, at least at the concentrations and durations tested. However, the presence of high levels of CR in MCS and other sources, as well as the lack of previous research, demonstrate the need for a better understanding of how CR exposure contributes to the cardiovascular pathology of real-world combustion-related exposures. Furthermore, the sex-dependence shown in relation to acute CR exposure supports the idea of sexdependence in the cardiovascular effects of unsaturated aldehyde exposures, an area that requires additional study. As exposures to acrolein and CR remain a daily occurrence for most of the global population, these studies confirm previous research and provide novel insight into the role of unsaturated aldehydes in the development of CVD, specifically implicating chronic CR exposure as a risk factor for CVD. 


\title{
CHAPTER VII
}

\author{
ROLES OF EXTRACELLULAR SUPEROXIDE DISMUTASE AND TRANSIENT \\ RECEPTOR POTENTIAL ANKYRIN 1 IN THE CARDIOVASCULAR EFFECTS OF \\ TOBACCO PRODUCTS AND CONSTITUENT ALDEHYDES
}

\section{A. Introduction}

One of the mechanisms proposed by Brook et al. (16) to explain how air pollutants may contribute to the development of cardiovascular disease (CVD) is the development of systemic oxidative stress and inflammation within the vasculature. In this hypothesis, oxidative stress results from overproduction of reactive oxygen species (ROS), which are highly reactive, oxygen-containing molecules $(16,40-43)$. ROS have been shown to play a beneficial role when maintained at normal levels (42-44), but excess production can lead to the development of oxidative stress $(40-42,44,47)$, which has been linked to incidence of CVD (42, 48-52). Mechanistically, the ROS-related development of CVD is at least in part mediated by changes in the bioavailability of nitric oxide (NO) and the development of endothelium dysfunction (40-42). The enzyme superoxide dismutase (SOD) indirectly plays an important role in regulating levels of NO in the blood; SOD catalyzes the dismutation of superoxide, but when the enzyme is inactivated, the free superoxide molecules can bind with NO and cause a decrease in free NO levels $(40,41,49,50,53)$. 
The subsequent imbalance of vasoactive factors in the vasculature can result in endothelium dysfunction $(40,41)$, which is one of the earliest vascular changes in a number of CVDs (54-58). Although ROS have been identified as therapeutic targets for the treatment of CVD (59-61), more research is needed to better understand the relationship between these vascular changes and disease development.

One of the many channels activated by ROS is the transient receptor potential ankyrin 1 (TRPA1) channel $(263-266,461,462)$. The TRPA1 channel has also been shown to be activated by a number of other stimuli, including physical factors $(260,262)$ and air pollutants including diesel exhaust, mainstream cigarette smoke (MCS), and aldehydes $(127,260,262,266,268-270)$. TRPA1 is primarily recognized as a pain $(262,272,279)$ or irritant $(262,264,279,286)$ receptor, but a number of other studies have demonstrated the role of TRPA1 in vasodilation $(127,260,265,266,270,272)$. Additionally, Conklin et al. (269) demonstrated that the TRPA1 channel plays an important role in the cardiopulmonary toxicity and lethality induced by high-level exposure to the aldehyde acrolein, and several other studies have also shown that the negative cardiovascular effects of inhaled acrolein are TRPA1-dependent $(261,289,290)$. However, the role of TRPA1 in the cardiovascular toxicity of other aldehydes, including crotonaldehyde (CR), is not well explored. Thus, the goal of these studies was to examine the roles of SOD, specifically extracellular SOD (ecSOD), and the TRPA1 channel in the mechanism through which constituents of personal air pollutant exposures mediate cardiovascular toxicity.

\section{B. Experimental Procedures}

\section{Exposures}

Animals were exposed to compounds or constituents via full-body inhalation as 
described in Chapter II, Sections B.2. Upon completion of the exposure, mice were euthanized and tissues collected as described in Chapter II, Section B.5.

\section{Urine Collection}

Urine samples were collected post-exposure as described in Chapter II, Section B.3.

\section{Urine Metabolite Quantification}

Collected urine was used to quantify levels of volatile organic compound (VOC) and tobacco alkaloid metabolites using analytical methods as described in Chapter II, Section B.4.

\section{Complete Blood Cell Count (CBC)}

A sample of whole blood was collected from each mouse after the final day of exposure for a complete blood cell count (CBC) as described in Chapter II, Section B.6.

\section{Plasma Biomarkers Quantification}

Plasma was prepared from each sample for an analysis of a number of biomarkers as described in Chapter II, Section B.7.

\section{Circulating Angiogenic Cell (CAC) Quantification}

Flow cytometry was used to analyze the number of circulating angiogenic cells (CACs) in each sample as described in Chapter II, Section B.8.

\section{Statistical Analyses}

Statistical analyses were performed as described in Chapter II, Section B.10.

\section{Results}

\section{Acute Exposure to MCS and the Role of TRPA1}


Because TRPA1 activation and neurogenic inflammation are induced by MCS, we performed acute exposures to MCS (4 days; $50 \%$ of smoke of $12 \mathrm{KY}$ Reference 3R4F cigarettes) using TRPA1-null male mice. Similar to the effects seen in MCS-exposed WT mice, TRPA-1 null mice showed a decreased final body weight $(-11 \% ; \mathrm{p}<0.001)$, but no changes in organ/body weight ratios (Table 7.1). Also similar to the WT mice, overall leukocyte count was significantly decreased with MCS exposure $(-51 \% ; \mathrm{p}<0.001)$, although this decrease was driven by decreases in both lymphocytes and monocytes (-34\%; $\mathrm{p}=0.004$ and $-40 \% ; \mathrm{p}=0.02$, respectively) (Table 7.2). MCS exposure otherwise had limited effects on $\mathrm{CBC}$ measures, inducing only a slight but significant increase in red cell distribution width $(+2 \% ; \mathrm{p}=0.03)$ and a modest decrease in platelet count $(-16 \% ; \mathrm{p}=0.06)$ (Table 7.2). Measures of liver (ALT, AST), muscle (CK), and LDH enzymes were unaffected by exposure, but exposed TRPA1-null mice showed a modest decrease in LDL $(-23 \% ; \mathrm{p}=0.05)$ and a modest increase in albumin $(+9 \% ; \mathrm{p}=0.07)$ (Table 7.1).

\section{Acute Exposure to Crotonaldehyde and the Role of TRPA1}

Because TRPA1 activation and neurogenic inflammation are induced by MCS and acrolein (261), we performed acute exposures to CR (4 days; 1 ppm) using TRPA1-null male mice. In contrast to the effects observed in CR-exposed male WT mice, TRPA1-null mice had no systemic changes in CBC (Table 7.3) yet did have a decreased kidney/body weight ratio $(-9 \% ; \mathrm{p}<0.001)$ relative to air-exposed control mice (Table 7.3). Plasma levels of ALT, AST, CK, and LDH enzymes, albumin, and creatinine were unchanged between the air- and CR-exposed TRPA1-null mice (Table 7.4). Cholesterol was decreased by CR exposure in the TRPA1-null mice (-17\%; $\mathrm{p}=0.003$; a change also observed in WT mice), a change due to decreased levels of LDL $(-49 \%$; $p<0.001)$ rather than in HDL level as 
Table 7.1. Systemic parameters of male TRPA1-null mice acutely exposed to either air or mainstream cigarette smoke (MCS; $50 \%$ of smoke of $12 \mathrm{KY}$ Reference 3R4F cigarettes).

\begin{tabular}{|c|c|c|}
\hline \multirow[b]{2}{*}{ Variable } & \multicolumn{2}{|c|}{ Exposure (4 days) } \\
\hline & Air Control & MCS \\
\hline Final BWT (g) & $33 \pm 0$ & $29 \pm 1 *_{\dagger}$ \\
\hline Heart/BWT (mg/g) & $5.5 \pm 0.1$ & $5.6 \pm 0.1 \dagger$ \\
\hline Lung/BWT (mg/g) & $6.1 \pm 0.2$ & $6.0 \pm 0.1$ \\
\hline Liver/BWT (mg/g) & $46.6 \pm 2.2$ & $47.7 \pm 2.2 \dot{\dagger}$ \\
\hline Kidney/BWT (mg/g) & $14.2 \pm 0.6$ & $13.4 \pm 0.4$ \\
\hline \multicolumn{3}{|c|}{ Plasma Measurements } \\
\hline Cholesterol (mg/dL) & $81.83 \pm 4.48$ & $78.00 \pm 3.57$ \\
\hline HDL (mg/dL) & $45.83 \pm 2.26$ & $48.29 \pm 3.41$ \\
\hline LDL (mg/dL) & $26.47 \pm 2.21$ & $20.34 \pm 0.78 \#$ \\
\hline Triglycerides (mg/dL) & $47.67 \pm 1.82 \dagger$ & $46.86 \pm 1.65 \dagger$ \\
\hline Albumin (g/dL) & $2.57 \pm 0.03 \dagger$ & $2.79 \pm 0.06 \# \dagger$ \\
\hline Total Protein (g/dL) & $5.07 \pm 0.11$ & $5.01 \pm 0.07$ \\
\hline ALT (U/I) & $12.67 \pm 2.16$ & $12.00 \pm 1.48 \dagger$ \\
\hline AST (U/I) & $44.17 \pm 3.49$ & $48.43 \pm 10.95$ \\
\hline CK (U/I) & $112.67 \pm 11.78$ & $144.73 \pm 52.39$ \\
\hline LDH (U/I) & $142.67 \pm 9.64 \dagger$ & $134.14 \pm 10.29 \dagger$ \\
\hline
\end{tabular}

Values $=$ mean \pm SE ( $=6-7$ mice per group); HDL \& LDL compared using Rank Sum Test. Abbr.: ALT, alanine aminotransferase; AST, aspartate aminotransferase; BWT, body weight; CK, creatine kinase; HDL, high-density lipoprotein; LDH, lactate dehydrogenase; LDL, low-density lipoprotein; MCS, mainstream cigarette smoke; TRPA1, transient receptor potential ankyrin 1; 
*, $\mathrm{p}<0.05$ compared with air control based on Bonferroni's or Dunn's post-test; \#, $0.05 \leq \mathrm{p} \leq 0.10$ compared with air control based on Bonferroni's or Dunn's post-test; †, p<0.05 for WT vs TRPA1-null based on Dunn's post-test. 
Table 7.2. Hematological measures of male TRPA1-null mice acutely exposed to either air or mainstream cigarette smoke (MCS;

$50 \%$ of smoke of $12 \mathrm{KY}$ Reference 3R4F cigarettes).

\begin{tabular}{|c|c|c|}
\hline & \multicolumn{2}{|c|}{ Exposure (4 days) } \\
\hline Hematological Measurements & Air Control & MCS \\
\hline White Blood Cell (K/uL) & $3.87 \pm 0.41 \dagger$ & $1.91 \pm 0.17 *_{\dagger}$ \\
\hline Neutrophils (K/uL) & $1.65 \pm 0.34$ & $0.46 \pm 0.04$ \\
\hline Lymphocytes (K/uL) & $2.13 \pm 0.20 \dagger$ & $1.40 \pm 0.15^{*} \dagger$ \\
\hline Monocytes (K/uL) & $0.09 \pm 0.01 \dagger$ & $0.05 \pm 0.01 *_{\mathbb{I}}$ \\
\hline Eosinophils (K/uL) & $0 \pm 0$ & $0 \pm 0$ \\
\hline Basophils (K/uL) & $0 \pm 0$ & $0 \pm 0$ \\
\hline Red Blood Cell (M/uL) & $8.91 \pm 0.24 \dagger$ & $8.88 \pm 0.19$ \\
\hline Hemoglobin (g/dL) & $13.1 \pm 0.3 \dagger$ & $13.3 \pm 0.2 \dagger$ \\
\hline Hematocrit (\%) & $44.0 \pm 1.0 \dagger$ & $44.9 \pm 1.0 \dagger$ \\
\hline Mean Corpuscular Volume (fL) & $49.5 \pm 0.8 \dagger$ & $50.6 \pm 0.3 \dagger$ \\
\hline Mean Corpuscular Hemoglobin (pg) & $14.7 \pm 0.3 \dagger$ & $15.0 \pm 0.1 \dagger$ \\
\hline Mean Corpuscular Hemoglobin Concentration (g/dL) & $29.7 \pm 0.4$ & $29.7 \pm 0.2 \dagger$ \\
\hline Red Cell Distribution Width (\%) & $18.5 \pm 0.6 \pi$ & $17.4 \pm 0.3 *$ \\
\hline Platelets $(\mathrm{K} / \mathrm{uL})$ & $880 \pm 108$ & $648 \pm 36 \#$ \\
\hline Mean Platelet Volume (fL) & $4.5 \pm 0$ & $4.3 \pm 0.1$ \\
\hline
\end{tabular}

Values = mean \pm SE (n=6-7 mice per group); Abbr.: MCS, mainstream cigarette smoke; TRPA1, transient receptor potential ankyrin $1 ;{ }^{*}, \mathrm{p}<0.05$ compared with air control based on Bonferroni's or Dunn's post-test; $\#, 0.05 \leq \mathrm{p} \leq 0.10$ compared with air control based on Bonferroni's or Dunn's post-test; †, p<0.05 for WT vs TRPA1-null based on Dunn's post-test; $\llbracket$, $0.05 \leq \mathrm{p} \leq 0.10$ for WT vs TRPA1-null based on Dunn's post-test. 
Table 7.3. Systemic measures in male TRPA1-null mice acutely exposed to either air or crotonaldehyde (CR; $1 \mathrm{ppm})$.

\begin{tabular}{|c|c|c|}
\hline \multirow[b]{2}{*}{ Variable } & \multicolumn{2}{|c|}{ Exposure Duration (4 days) } \\
\hline & Air Control & CR (1 ppm) \\
\hline Change in BWT (g) & $-1 \pm 1$ & $-1 \pm 0$ \\
\hline Heart/BWT (mg/g) & $5.3 \pm 0.2$ & $5.0 \pm 0.2$ \\
\hline Lung/BWT (mg/g) & $5.7 \pm 0.2$ & $5.4 \pm 0.2$ \\
\hline Liver/BWT (mg/g) & $47.8 \pm 2.0 \dagger$ & $47.8 \pm 1.0 \dagger$ \\
\hline Kidney/BWT (mg/g) & $14.2 \pm 0.4 \dagger$ & $12.9 \pm 0.2 *$ \\
\hline \multicolumn{3}{|l|}{ Hematological Measurements } \\
\hline White Blood Cell $(\mathrm{K} / \mu \mathrm{L})$ & $1.70 \pm 0.24$ & $1.57 \pm 0.27$ \\
\hline Neutrophils $(\mathrm{K} / \mu \mathrm{L})$ & $0.59 \pm 0.12$ & $0.47 \pm 0.09$ \\
\hline Lymphocytes $(\mathrm{K} / \mu \mathrm{L})$ & $1.06 \pm 0.12$ & $1.06 \pm 0.18$ \\
\hline Monocytes $(\mathrm{K} / \mu \mathrm{L})$ & $0.05 \pm 0.01$ & $0.08 \pm 0.04$ \\
\hline Eosinophils $(\mathrm{K} / \mu \mathrm{L})$ & $0 \pm 0$ & $0 \pm 0$ \\
\hline Basophils $(\mathrm{K} / \mu \mathrm{L})$ & $0 \pm 0$ & $0 \pm 0$ \\
\hline Red Blood Cell $(\mathrm{M} / \mu \mathrm{L})$ & $8.40 \pm 0.13$ & $8.72 \pm 0.10$ \\
\hline Hemoglobin (g/dL) & $11.6 \pm 0.2$ & $12.1 \pm 0.1$ \\
\hline Hematocrit (\%) & $37.8 \pm 0.7$ & $39.5 \pm 0.4$ \\
\hline Mean Corpuscular Volume (fL) & $45.0 \pm 0.3$ & $45.3 \pm 0.3 \dagger$ \\
\hline Mean Corpuscular Hemoglobin (pg) & $13.9 \pm 0.1$ & $13.8 \pm 0.1$ \\
\hline Mean Corpuscular Hemoglobin Concentration (g/dL) & $30.8 \pm 0.2$ & $30.6 \pm 0.3$ \\
\hline Red Cell Distribution Width (\%) & $18.3 \pm 0.4 \dagger$ & $17.7 \pm 0.3$ \\
\hline Platelets $(\mathrm{K} / \mu \mathrm{L})$ & $869 \pm 51 \dagger$ & $862 \pm 56 \dagger$ \\
\hline Mean Platelet Volume (fL) & $4.5 \pm 0.1 \dagger$ & $4.6 \pm 0.1 \dagger$ \\
\hline
\end{tabular}

Values = mean \pm SE (n=9-15 mice per group); Abbr.: BWT, body weight; CR, crotonaldehyde; TRPA1, transient receptor potential ankyrin 1; *, p<0.05 compared with air control based on Bonferroni’s or Dunn's post-test; $\dagger, \mathrm{p}<0.05$ for WT vs TRPA1-null based on 
Dunn's post-test. 
Table 7.4. Plasma biomarkers in male TRPA1-null mice acutely exposed to either air or crotonaldehyde (CR; 1 ppm).

\begin{tabular}{|l|c|c|}
\hline \multicolumn{1}{|c|}{ Plasma Measurements } & \multicolumn{2}{c|}{ Exposures (4 days) } \\
\hline Cholesterol (mg/dL) & Air Control & CR (1 ppm) \\
\hline HDL (mg/dL) & $81.39 \pm 3.13 \dagger$ & $67.27 \pm 3.48^{*}$ \\
\hline LDL (mg/dL) & $49.95 \pm 2.89$ & $47.34 \pm 2.80$ \\
\hline Triglycerides (mg/dL) & $8.73 \pm 0.96 \dagger$ & $4.49 \pm 0.52^{*}$ \\
\hline Albumin (g/dL) & $40.77 \pm 3.60$ & $27.39 \pm 1.66$ \\
\hline Total Protein (g/dL) & $2.75 \pm 0.10$ & $2.77 \pm 0.07$ \\
\hline ALT (U/I) & $5.11 \pm 0.17 \dagger$ & $4.83 \pm 0.11 \dagger$ \\
\hline AST (U/I) & $28.91 \pm 1.43$ & $33.71 \pm 5.31$ \\
\hline CK (U/I) & $70.62 \pm 4.13$ & $69.88 \pm 8.82$ \\
\hline LDH (U/I) & $222.70 \pm 27.59$ & $233.81 \pm 31.69$ \\
\hline Creatinine (mg/dL) & $138.03 \pm 7.35$ & $160.28 \pm 12.70$ \\
\hline
\end{tabular}

Values = mean \pm SE (n=5-6 mice per group); Abbr.: ALT, alanine aminotransferase; AST, aspartate aminotransferase; CK, creatine kinase; CR, crotonaldehyde; HDL, high-density lipoprotein; LDH, lactate dehydrogenase; LDL, low-density lipoprotein; TRPA1, transient receptor potential ankyrin 1 ; $^{*}, \mathrm{p}<0.05$ compared with air control based on Bonferroni’s or Dunn's post-test; \#, $0.05 \leq \mathrm{p} \leq 0.10$ compared with air control based on Bonferroni's or Dunn's post-test; †, $\mathrm{p}<0.05$ for WT vs TRPA1-null based on Dunn's post-test. 
observed in WT mice (Table 7.4). As with the WT mice, TRPA1-null mice acutely exposed to 1 ppm CR had no change in the levels of circulating Sca-1+ cells or Flk-1+/Sca1+ cells (Fig. 7.1A \& B).

\section{Urinary Metabolites in MCS-Exposed WT and ecSOD-Tg Mice}

The antioxidant enzyme superoxide dismutase (SOD) catalyzes the dismutation of superoxide molecules to prevent their binding with nitric oxide and the subsequent formation of peroxynitrite $(40-42,53)$. Peroxynitrite can disrupt the proper production of nitric oxide, which contributes to the development of endothelium dysfunction $(40,41)$. This dysfunction is one of the early vascular changes noted in a number of CVDs (54-57), but increased levels of SOD, particularly extracellular SOD (ecSOD), have been shown to be protective in cardiac tissues (463). To better understand the role of ecSOD in the cardiovascular-related effects of MCS, we measured urinary metabolites by UPLC-MS/MS and GC-MS/MS in mice exposed to a known number of cigarettes. For this, male mice overexpressing ecSOD (ecSOD-Tg; Tg) and their wild type littermates (WT) were exposed to MCS (50\% of smoke of 12 or $6 \mathrm{KY}$ Reference 3R4F cigarettes, 6h/day, 4d), and urine was immediately collected after the exposure sessions. Urinary levels of 3hydroxypropylmercapturic acid (3HPMA) were not significantly different between WT and Tg mice at baseline (Fig. 7.2A). Likewise, levels of 3HPMA after the first three hours post-MCS exposure were not significantly different from baseline, nor were levels different between background (WT or Tg) or between number of cigarettes (12 or 6) (Fig. 7.2A). Conversely, urinary HPMMA levels were significantly increased in both WT $(\mathrm{p}=0.02)$ and $\operatorname{Tg}(\mathrm{p}=0.003)$ mice exposed to 12 cigarettes and WT $(\mathrm{p}=0.02)$ and $\mathrm{Tg}(\mathrm{p}=0.006)$ mice exposed to 6 cigarettes compared with baseline (Fig 7.2B). However, there were again no 


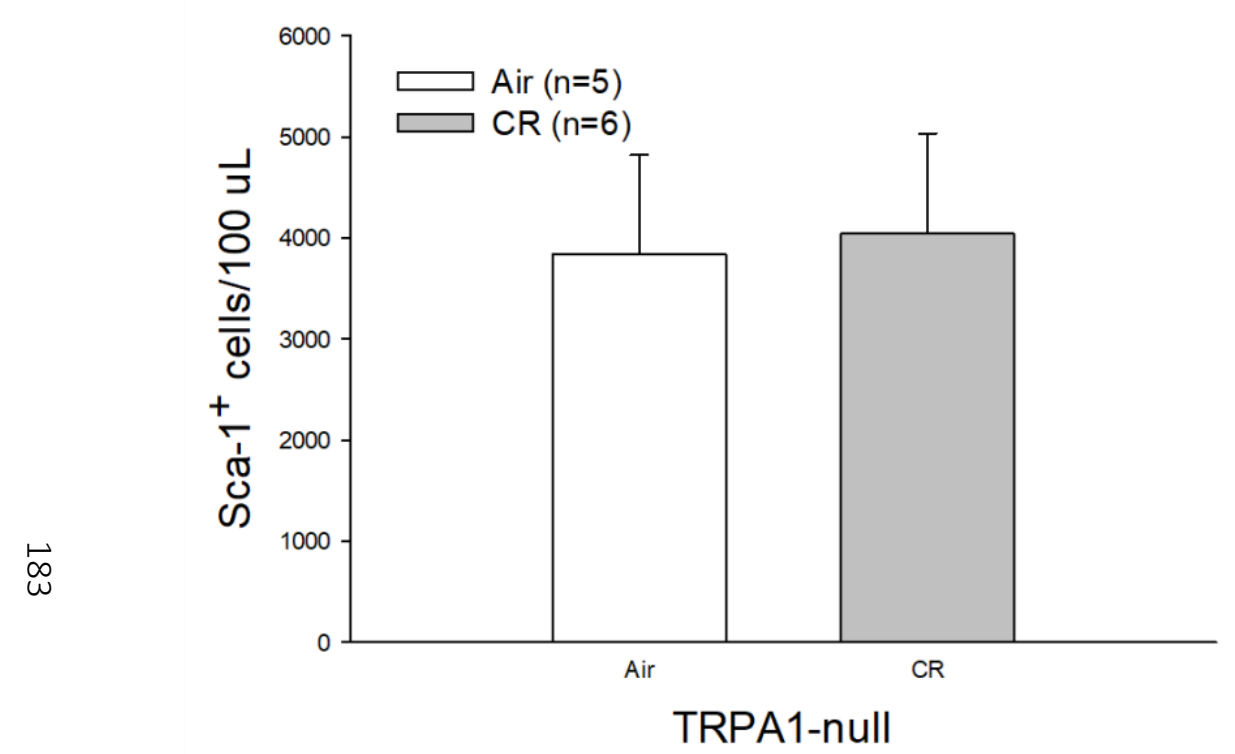

B.

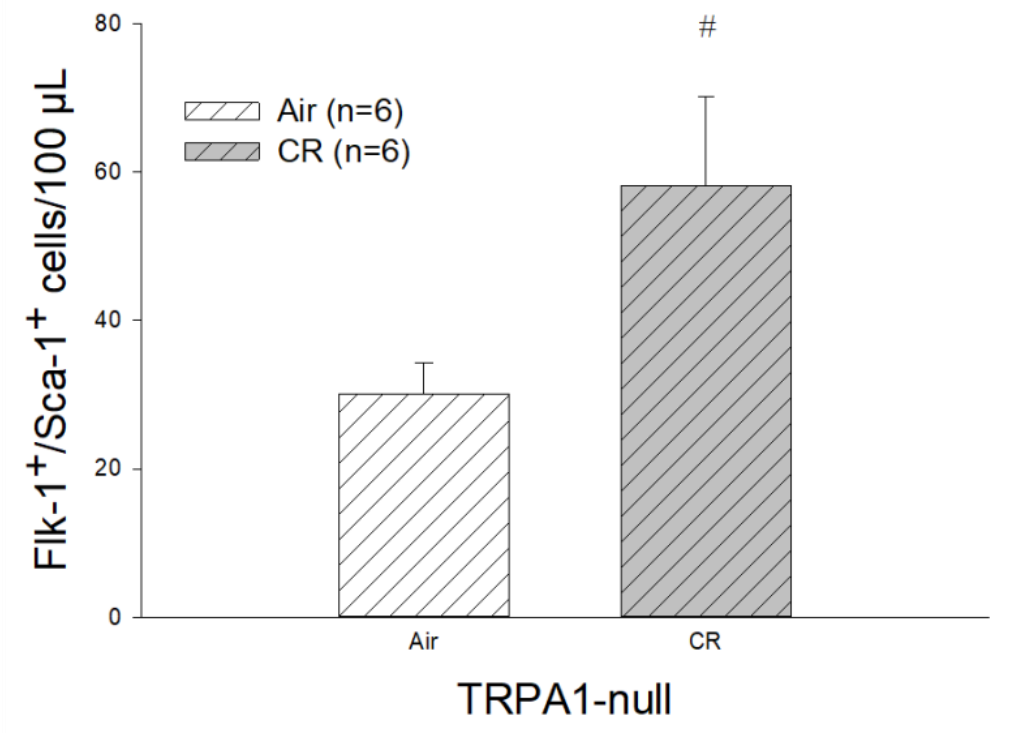

Fig. 7.1. Effects of acute crotonaldehyde (CR; 1 ppm) exposure on circulating stem cells in male TRPA1-null mice.

Hematopoietic stem cells (Sca-1+) and circulating angiogenic cells (CACs) were measured by flow cytometry in male TRPA1-null male mice exposed to HEPA-filtered air or CR (1 ppm, 6h/d) for 4 days. After 4 days of exposure to CR, TRPA1-null mice had no change in the level of Sca-1+ cells (A) but had a modest increase in the level of CACs (Flk-1+/Sca-1+ cells (B) compared with airexposed control. Values $=$ mean $\pm \operatorname{SE}(n=5-6$ mice per group $) ; \#, 0.05 \leq p \leq 0.10$ vs air control. 

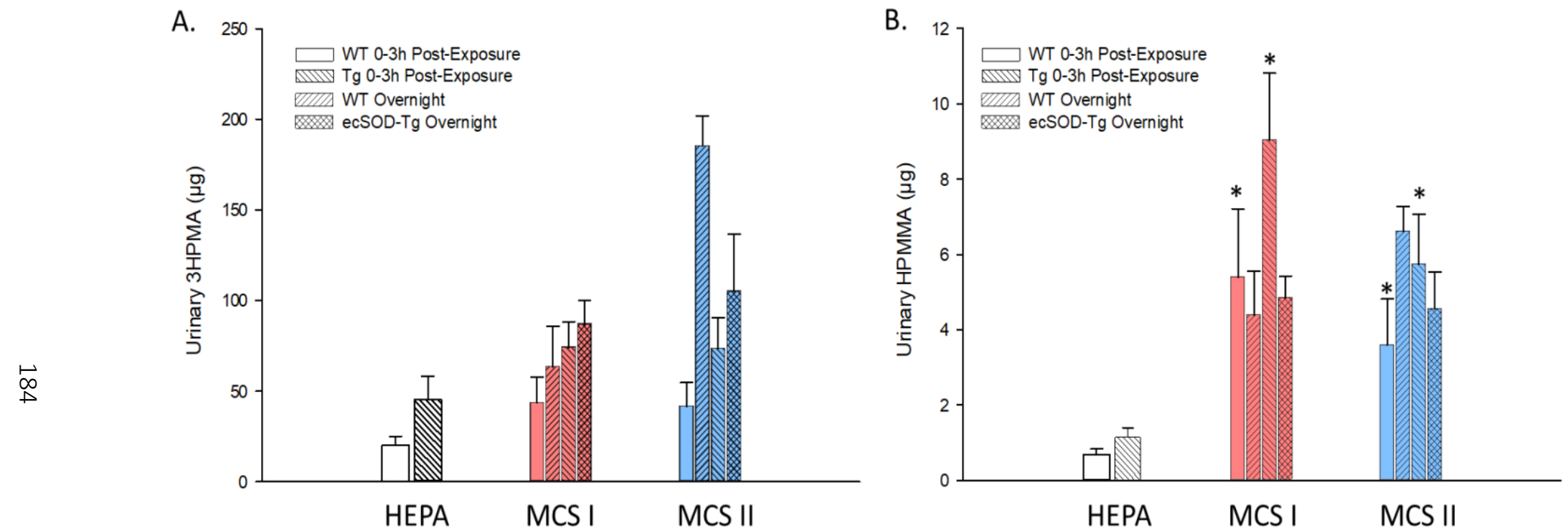

Fig. 7.2. Urinary 3HPMA \& HPMMA levels after acute mainstream cigarette smoke (MCS) exposure. Male WT and ecSOD-Tg mice were exposed (6h) to mainstream cigarette smoke (MCS; MCS I: 50\% of smoke of 12 KY Reference 3R4F cigarettes; MCS II: $50 \%$ of smoke of $6 \mathrm{KY}$ Reference 3R4F cigarettes) after which urine was collected at $0-1,1-2,2-3$, and 3-16h (overnight) and 3hydroxypropylmercapturic acid (3HPMA; A) and 3-hydroxy-1-methylpropylmercapturic acid (HPMMA; B) quantified. Values are mean \pm SE ( $n=3-5$ mice per group). Abbr.: ecSOD-Tg; extracellular superoxide dismutase transgenic; HEPA, high-efficiency 
particulate air; 3HPMA, 3-hydroxypropylmercapturic acid; HPMMA, 3-hydroxy-1-methylpropylmercapturic acid; MCS, mainstream cigarette smoke; WT, wild type. *, p<0.05 compared with same strain HEPA control. 
significant differences in HPMMA between WT and Tg mice exposed to the same number of cigarettes (Fig. 7.2B). Levels of total urinary nicotine metabolites were below the limit of detection in HEPA-exposed mice, and mice exposed to either 6 or 12 cigarettes showed significant increases in the first three hours post-exposure regardless of background (WT: $\mathrm{p}=0.02$ for both; $\mathrm{Tg}$ : $\mathrm{p}<0.001$ for both) (Fig. 7.3). Levels were not different, however, between mice of the same background exposed to the same number of cigarettes (Fig. 7.3).

\section{Acute Exposure to MCS and the Role of ecSOD}

Because of the protective cardiopulmonary effects seen with overexpression of the antioxidant enzyme ecSOD (463-465), we tested for the potential benefit of this overexpression in biomarkers of systemic and hematological injury in mice exposed to MCS (50\% of smoke of 6 or 12 3R4F KY reference cigarettes). Tg mice acutely (4d) exposed to 6 cigarettes showed a greater final body weight compared with their 6 cigaretteexposed WT littermates $(+6 \% ; \mathrm{p}=0.07)$ (Table 7.5), while no change was seen between WT and Tg mice exposed to 12 cigarettes (Table 7.6). The lung/body weight ratio, however, was significantly lower in $\mathrm{Tg}$ mice exposed to 12 cigarettes compared with 12 cigarette-exposed WT mice $(-20 \% ; \mathrm{p}=0.02)$ (Table 7.6); only the liver/body weight ratio was different between WT and Tg mice exposed to 6 cigarettes, with Tg mice showing a modestly lower ratio $(-7 \% ; \mathrm{p}=0.07)$ (Table 7.5$)$. Acute exposure showed minimal differences in both CBC and plasma biomarkers between WT and Tg mice exposed to the same number of cigarettes but considerable differences in mice of the same background exposed to a different number of cigarettes. For example, in mice exposed to 6 cigarettes, lymphocyte counts were lower in $\mathrm{Tg}$ mice $(-23 \% ; \mathrm{p}=0.08)$ (Table 7.5), but no differences were noted in mice exposed to 12 cigarettes (Table 7.6). However, both WT and Tg mice 


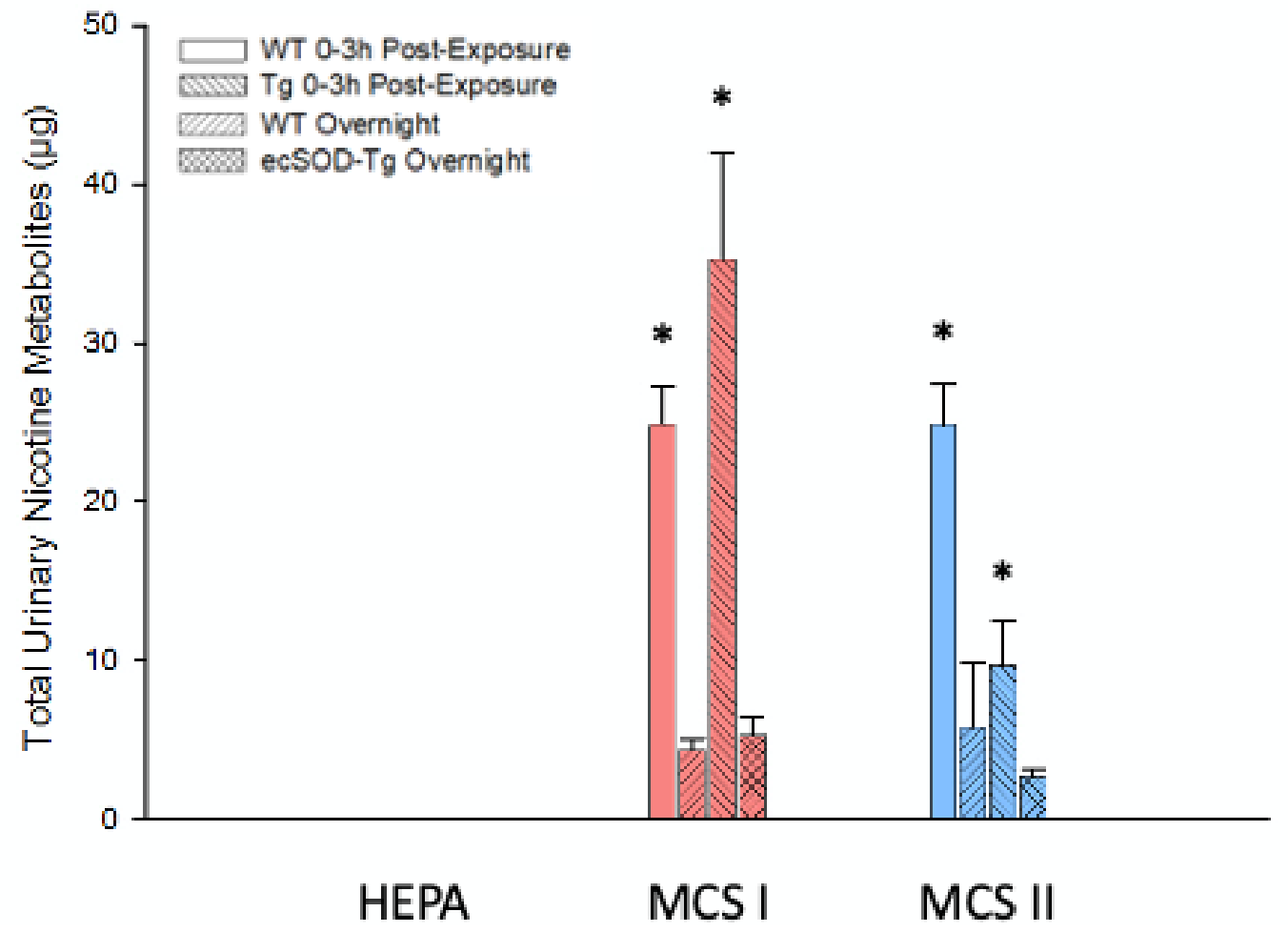

Fig. 7.3. Total urinary nicotine metabolite levels after acute mainstream cigarette smoke (MCS) exposure. Male WT and ecSOD-Tg mice were exposed (6h) to mainstream cigarette smoke (MCS; MCS I: 50\% of 12 KY Reference 3R4F cigarettes; MCS II: 50\% of smoke of $6 \mathrm{KY}$ Reference 3R4F cigarettes) after which urine was collected at $0-1,1-2$, $2-3$, and $3-16 \mathrm{~h}$ (overnight) and total urinary nicotine metabolites (nicotine, cotinine, and trans-3'-hydroxycotinine) quantified. Values are mean $\pm \mathrm{SE}$ ( $\mathrm{n}=3-5$ mice per group). Abbr.: ecSOD-Tg; extracellular superoxide dismutase transgenic; HEPA, high-efficiency particulate air; MCS, mainstream cigarette smoke; WT, wild type. ${ }^{*}, \mathrm{p}<0.05$ compared with same strain HEPA control. 
Table 7.5. Systemic changes in male WT or ecSOD-Tg mice acutely exposed to either air or mainstream cigarette smoke (MCS; $50 \%$ of smoke of $6 \mathrm{KY}$ Reference 3R4F cigarettes).

\begin{tabular}{|c|c|c|}
\hline \multirow[b]{2}{*}{ Variable } & \multicolumn{2}{|c|}{ MCS (6 cigs, 4d) } \\
\hline & $\mathbf{W T}$ & ecSOD-Tg \\
\hline Final BWT (g) & $30 \pm 1$ & $32 \pm 1 \#$ \\
\hline Heart/BWT (mg/g) & $5.4 \pm 0.3$ & $4.7 \pm 0.1$ \\
\hline Lung/BWT (mg/g) & $5.3 \pm 0.3$ & $5.1 \pm 0.1$ \\
\hline Liver/BWT (mg/g) & $42.7 \pm 1.1$ & $39.7 \pm 0.6 \#$ \\
\hline \multicolumn{3}{|l|}{ Hematological Measurements } \\
\hline White Blood Cell $(\mathrm{K} / \mu \mathrm{L})$ & $3.83 \pm 0.63 \dagger$ & $3.55 \pm 0.51 \llbracket$ \\
\hline Neutrophils $(\mathrm{K} / \mu \mathrm{L})$ & $1.27 \pm 0.53$ & $1.55 \pm 0.49 \mathbb{I}$ \\
\hline Lymphocytes $(\mathrm{K} / \mu \mathrm{L})$ & $2.48 \pm 0.34 \dagger$ & $1.91 \pm 0.19$ 折 \\
\hline Monocytes $(\mathrm{K} / \mu \mathrm{L})$ & $0.07 \pm 0.01$ & $0.08 \pm 0.02 \pi$ \\
\hline Eosinophils $(\mathrm{K} / \mu \mathrm{L})$ & $0.01 \pm 0$ & $0.01 \pm 0.01$ \\
\hline Basophils $(\mathrm{K} / \mu \mathrm{L})$ & $0 \pm 0$ & $0 \pm 0$ \\
\hline Red Blood Cell $(\mathrm{M} / \mu \mathrm{L})$ & $8.35 \pm 0.35$ & $8.54 \pm 0.16 \llbracket$ \\
\hline Hemoglobin (g/dL) & $12.3 \pm 0.9$ & $12.6 \pm 0.5 \llbracket$ \\
\hline Hematocrit (\%) & $36.3 \pm 1.4$ & $37.3 \pm 0.8 \llbracket$ \\
\hline Mean Corpuscular Volume (fL) & $43.5 \pm 0.2$ & $43.7 \pm 0.2$ \\
\hline Mean Corpuscular Hemoglobin (pg) & $14.7 \pm 0.6$ & $14.8 \pm 0.4$ \\
\hline Mean Corpuscular Hemoglobin Concentration (g/dL) & $33.8 \pm 1.5$ & $33.9 \pm 0.7$ \\
\hline Red Cell Distribution Width (\%) & $18.2 \pm 0.2$ & $18.0 \pm 0.2$ \\
\hline Platelets $(\mathrm{K} / \mu \mathrm{L})$ & $776 \pm 57$ & $788 \pm 24$ \\
\hline Mean Platelet Volume (fL) & $4.7 \pm 0$ & $4.7 \pm 0$ \\
\hline
\end{tabular}

Values = mean \pm SE (n=5 mice per group); Abbr.: BWT, body weight; ecSOD, extracellular superoxide dismutase; ecSOD-Tg, 
extracellular superoxide dismutase transgenic; MCS, mainstream cigarette smoke; WT, wild type; *, p<0.05 compared with WT based on Bonferroni's or Dunn's post-test; \#, $0.05 \leq \mathrm{p} \leq 0.10$ compared with WT based on Bonferroni’s or Dunn's post-test; $\uparrow, 0.05 \leq \mathrm{p} \leq 0.10$ for 12 vs 6 cigarettes in WT mice based on Dunn’s post-test; đI, $0.05 \leq \mathrm{p} \leq 0.10$ for 12 vs 6 cigarettes in ecSOD-Tg mice based on Dunn’s posttest. 
Table 7.6. Systemic changes in male WT or ecSOD-Tg mice acutely exposed to either air or mainstream cigarette smoke (MCS; $50 \%$ of smoke of $12 \mathrm{KY}$ Reference 3R4F cigarettes).

\begin{tabular}{|c|c|c|}
\hline \multirow[b]{2}{*}{ Variable } & \multicolumn{2}{|c|}{ MCS (12 cigs, 4d) } \\
\hline & $\mathbf{W T}$ & ecSOD-Tg \\
\hline Final BWT (g) & $31 \pm 0$ & $32 \pm 1$ \\
\hline Heart/BWT (mg/g) & $4.8 \pm 0.2$ & $4.1 \pm 0.5$ \\
\hline Lung/BWT (mg/g) & $6.1 \pm 0.3$ & $4.8 \pm 0.5^{*}$ \\
\hline Liver/BWT (mg/g) & $39.9 \pm 1.4$ & $40.9 \pm 1.1$ \\
\hline \multicolumn{3}{|l|}{ Hematological Measurements } \\
\hline White Blood Cell $(\mathrm{K} / \mu \mathrm{L})$ & $1.38 \pm 0.24$ & $1.25 \pm 0.21$ \\
\hline Neutrophils $(\mathrm{K} / \mu \mathrm{L})$ & $0.43 \pm 0.12$ & $0.40 \pm 0.05$ \\
\hline Lymphocytes $(\mathrm{K} / \mu \mathrm{L})$ & $0.90 \pm 0.11$ & $0.81 \pm 0.19$ \\
\hline Monocytes $(\mathrm{K} / \mu \mathrm{L})$ & $0.04 \pm 0.01$ & $0.04 \pm 0.01$ \\
\hline Eosinophils $(\mathrm{K} / \mu \mathrm{L})$ & $0 \pm 0$ & $0 \pm 0$ \\
\hline Basophils $(\mathrm{K} / \mu \mathrm{L})$ & $0 \pm 0$ & $0 \pm 0$ \\
\hline Red Blood Cell $(\mathrm{M} / \mu \mathrm{L})$ & $7.66 \pm 0.39$ & $7.26 \pm 0.37$ \\
\hline Hemoglobin (g/dL) & $10.8 \pm 0.3$ & $10.3 \pm 0.7$ \\
\hline Hematocrit (\%) & $33.5 \pm 1.7$ & $31.6 \pm 1.8$ \\
\hline Mean Corpuscular Volume (fL) & $43.7 \pm 0.1$ & $43.5 \pm 0.3$ \\
\hline Mean Corpuscular Hemoglobin (pg) & $14.1 \pm 0.4$ & $14.1 \pm 0.3$ \\
\hline Mean Corpuscular Hemoglobin Concentration (g/dL) & $32.3 \pm 0.9$ & $32.2 \pm 0.7$ \\
\hline Red Cell Distribution Width (\%) & $18.6 \pm 0.4$ & $18.0 \pm 0.3$ \\
\hline Platelets $(\mathrm{K} / \mu \mathrm{L})$ & $823 \pm 66$ & $826 \pm 45$ \\
\hline Mean Platelet Volume (fL) & $4.6 \pm 0.1$ & $4.6 \pm 0.1$ \\
\hline
\end{tabular}

Values = mean \pm SE (n=5 mice per group); Abbr.: BWT, body weight; ecSOD, extracellular superoxide dismutase; ecSOD-Tg, 
extracellular superoxide dismutase transgenic; MCS, mainstream cigarette smoke; WT, wild type; *, p<0.05 compared with ecSODWT based on Bonferroni's or Dunn's post-test; \#, $0.05 \leq \mathrm{p} \leq 0.10$ compared with WT based on Bonferroni's or Dunn's post-test; $\uparrow, \mathrm{p}<0.05$ for 12 vs 6 cigarettes in WT mice based on Dunn’s post-test; q, $0.05 \leq \mathrm{p} \leq 0.10$ for 12 vs 6 cigarettes in ecSOD-Tg mice based on Dunn’s post-test. 
showed higher levels of leukocytes after exposure to 6 cigarettes compared with levels after exposure to 12 cigarettes $(+178 \%$; $\mathrm{p}=0.002$ and $+185 \%$; $\mathrm{p}=0.001$, respectively) (Tables $7.5 \& 7.6)$. Likewise, lymphocytes were higher in both WT $(+174 \%$; $p<0.001)$ and $\operatorname{Tg}(+137 \% ; \mathrm{p}=0.001)$ mice after exposure to 6 cigarettes than after exposure to 12 cigarettes, while neutrophils $(+289 \%$; $\mathrm{p}=0.04)$ and monocytes $(+90 \% ; \mathrm{p}=0.07)$ were only higher in Tg mice (Tables 7.5 \& 7.6). MCS seemed to induce a greater response mice in erythrocytic measures of $\mathrm{Tg}$ mice, with differences noted in red blood cell count $(+18 \%$; $\mathrm{p}=0.01)$ and measures of hemoglobin $(+23 \% ; \mathrm{p}=0.01)$ and hematocrit $(+18 \% ; \mathrm{p}=0.01)$ between Tg mice exposed to 12 or 6 cigarettes (Tables $7.5 \&$ 7.6). Acute exposure to MCS (6 or 12 cigarettes) did not affect plasma levels of CK (striated muscle), ALT or AST (liver), or LDH (non-specific cell toxicity) enzymes in either WT or Tg mice (Tables 7.7 \& 7.8), but a number of differences were once again noted between mice of the same background exposed to a different number of cigarettes. Cholesterol was modestly higher in both WT $(+16 \%$; $\mathrm{p}=0.07)$ and $\operatorname{Tg}(+13 \% ; \mathrm{p}=0.08)$ mice exposed to 6 cigarettes compared with mice exposed to 12 cigarettes (Tables $7.7 \& 7.8$ ); both albumin (WT: $+17 \%, \mathrm{p}=0.02$; Tg: $+10 \%, \mathrm{p}=0.09)$ and total protein (WT: $+16 \%, \mathrm{p}=0.01 ; \mathrm{Tg}:+15 \%, \mathrm{p}=0.01)$ showed this same pattern, while LDL was higher in only Tg mice exposed to 6 cigarettes compared with $\mathrm{Tg}$ mice exposed to 12 cigarettes $(+16 \%$; $\mathrm{p}=0.08)$ (Tables $7.7 \& 7.8)$. No differences

were seen in levels of Sca-1+ cells or CACs regardless of background or exposure (Fig. $7.5 \mathrm{~A} \& \mathrm{~B})$.

\section{Discussion}

Studies have shown MCS to be both a source of ROS (466-470) and a promoter of ROS production $(471,472)$, and the subsequent development of oxidative stress is one 
Table 7.7. Plasma biomarkers in male WT or ecSOD-Tg mice acutely exposed to either air or mainstream cigarette smoke (50\% of smoke of MCS; 6 KY Reference 3R4F cigarettes).

\begin{tabular}{|l|c|c|}
\hline \multicolumn{1}{|c|}{ Plasma Measurements } & \multicolumn{2}{c|}{ MCS (6 cigs, 4d) } \\
\hline Cholesterol (mg/dL) & ecSOD-WT & ecSOD-Tg \\
\hline HDL (mg/dL) & $85.70 \pm 5.02_{\dagger}$ & $85.55 \pm 3.96 \pi$ \\
\hline LDL (mg/dL) & $35.81 \pm 2.68$ & $37.56 \pm 2.79$ \\
\hline Triglycerides (mg/dL) & $32.62 \pm 1.98$ & $32.81 \pm 1.87 \pi$ \\
\hline Albumin (g/dL) & $49.70 \pm 5.88$ & $51.68 \pm 2.63$ \\
\hline Total Protein (g/dL) & $3.17 \pm 0.17 \dagger$ & $2.98 \pm 0.08_{\llbracket}$ \\
\hline ALT (U/I) & $5.26 \pm 0.23_{\dagger}$ & $5.11 \pm 0.08_{\llbracket}$ \\
\hline AST (U/I) & $27.58 \pm 4.61_{\dagger}$ & $27.02 \pm 2.95 \pi$ \\
\hline CK (U/I) & $63.36 \pm 14.19$ & $67.20 \pm 4.75$ \\
\hline LDH (U/I) & $260.89 \pm 111.04$ & $333.57 \pm 25.98$ \\
\hline Creatinine (mg/dL) & $218.80 \pm 44.05$ & $260.40 \pm 30.58$ \\
\hline
\end{tabular}

Values = mean \pm SE ( $n=4-6$ mice per group); Abbr.: ecSOD, extracellular superoxide dismutase; ecSOD-Tg, extracellular superoxide dismutase transgenic; HDL, high-density lipoprotein; LDL, low-density lipoprotein; MCS, mainstream cigarette smoke; WT, wild type; *, $\mathrm{p}<0.05$ compared with WT based on Bonferroni's or Dunn's post-test; \#, $0.05 \leq \mathrm{p} \leq 0.10$ compared with WT based on Bonferroni's or Dunn's post-test; †, $\mathrm{p}<0.05$ for 12 vs 6 cigarettes in WT mice based on Dunn's post-test; ql, $0.05 \leq \mathrm{p} \leq 0.10$ for 12 vs 6 cigarettes in ecSOD-Tg mice based on Dunn's post-test. 
Table 7.8. Plasma biomarkers in male ecSOD-WT or ecSOD-Tg mice acutely exposed to either air or mainstream cigarette smoke (MCS; 50\% of smoke of 12 KY Reference 3R4F cigarettes).

\begin{tabular}{|l|c|c|}
\hline \multicolumn{1}{|c|}{ Plasma Measurements } & \multicolumn{2}{c|}{ MCS (12 cigs, 4d) } \\
\hline Cholesterol (mg/dL) & ecSOD-WT & ecSOD-Tg \\
\hline HDL (mg/dL) & $73.91 \pm 3.20$ & $75.42 \pm 4.09$ \\
\hline LDL (mg/dL) & $33.10 \pm 1.32$ & $33.15 \pm 1.84$ \\
\hline Triglycerides (mg/dL) & $28.93 \pm 1.29$ & $28.33 \pm 1.83$ \\
\hline Albumin (g/dL) & $41.86 \pm 8.75$ & $38.89 \pm 3.44$ \\
\hline Total Protein (g/dL) & $2.72 \pm 0.10$ & $2.71 \pm 0.11$ \\
\hline ALT (U/I) & $4.54 \pm 0.16$ & $4.43 \pm 0.18$ \\
\hline AST (U/I) & $17.03 \pm 1.19$ & $19.95 \pm 0.69$ \\
\hline CK (U/I) & $49.35 \pm 4.89$ & $55.83 \pm 4.82$ \\
\hline LDH (U/I) & $201.59 \pm 29.92$ & $252.97 \pm 39.54$ \\
\hline Creatinine (mg/dL) & $178.21 \pm 16.26$ & $202.75 \pm 19.31$ \\
\hline
\end{tabular}

Values = mean \pm SE (n=4-6 mice per group); Abbr.: ecSOD, extracellular superoxide dismutase; ecSOD-Tg, extracellular superoxide dismutase transgenic; HDL, high-density lipoprotein; LDL, low-density lipoprotein; MCS, mainstream cigarette smoke; WT, wild type; *, $\mathrm{p}<0.05$ compared with WT based on Bonferroni's or Dunn's post-test; $\#, 0.05 \leq \mathrm{p} \leq 0.10$ compared with WT based on Bonferroni’s or Dunn's post-test; $\uparrow, \mathrm{p}<0.05$ for 12 vs 6 cigarettes in WT mice based on Dunn’s post-test; $\llbracket$, $0.05 \leq \mathrm{p} \leq 0.10$ for 12 vs 6 cigarettes in ecSOD-Tg mice based on Dunn's post-test. 

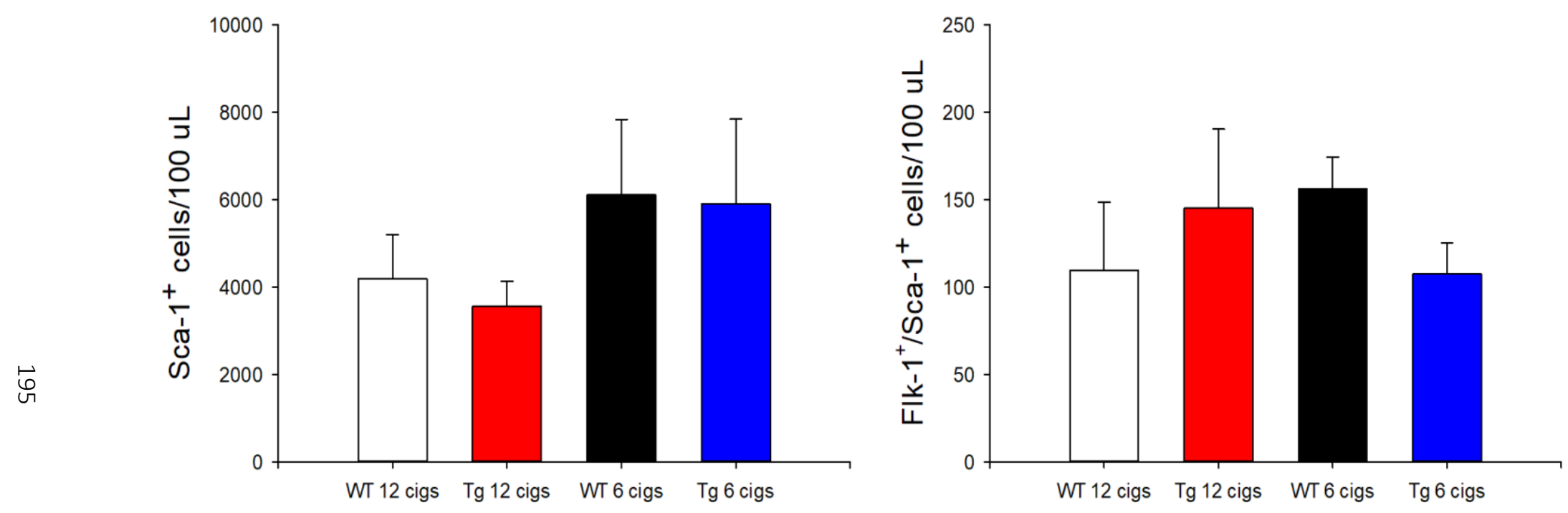

Fig. 7.4. Effects of acute mainstream cigarette smoke (MCS; 6 or 12 cigarettes) exposure on circulating stem cells in male WT and ecSOD-Tg mice. Hematopoietic stem cells (Sca-1+) and circulating angiogenic cells (CACs) were measured by flow cytometry in male WT and ecSOD-Tg male mice exposed to HEPA-filtered air or MCS (6 or 12 cigs; 6h/d) for 4 days. After 4 days of exposure to MCS, there were no changes in the level of Sca-1+ cells $(\mathbf{A})$ or CACs $(\mathbf{B})$ regardless of background or the number of cigarettes. Values $=$ mean $\pm \mathrm{SE}(\mathrm{n}=4-6$ mice per group $)$. 
mechanism through which MCS contributes to the development of CVD (467, 471-474). The SOD enzyme serves to dismutate the superoxide radical and thus works to attenuate the development of oxidative stress in the vasculature. Studies of both under- and overexpression of ecSOD have demonstrated the role of this enzyme in the development of vascular changes and injury that could contribute to CVD and other smoking-related diseases $(299,475-478)$. The TRPA1 channel is a non-selective, cation channel and a promiscuous receptor concentrated in sensory fibers that mediates pain reception/transmission as well as irritant-related pulmonary reflexes including cough and bronchoconstriction in response to unsaturated aldehydes generated in MCS (261). TRPA1 can also be activated by $\operatorname{ROS}(263-266,461,462)$, which may also contribute to the channel's role in the development of CVD. The goal of the current studies was to explore the roles of ecSOD and the TRPA1 channel through the use of transgenic (ecSOD-Tg mice and TRPA1-null) mice in response to MCS and CR exposures.

Male ecSOD-Tg mice and their WT littermates were exposed to MCS generated from 6 or $12 \mathrm{KY}$ Reference 3R4F cigarettes. Based on the overall data, there was no discernable indication that ecSOD-Tg mice were better protected from MCS-generated aldehydes than their similarly-exposed WT counterparts through increased levels of pulmonary ecSOD. Urinary measures of acrolein and crotonaldehyde metabolites showed no significant differences between WT and Tg mice exposed to either 6 or 12 cigarettes. There were also no significant differences in measures of urinary nicotine between WT and $\mathrm{Tg}$ mice, and despite one set of mice being exposed to smoke from twice as many cigarettes, levels of nicotine were similar between the two exposures in both strains of mice. 
Measures of systemic injury assessed through $\mathrm{CBC}$ and measures of plasma biomarkers showed minimal differences between similarly-exposed WT and Tg mice, but there were a number of significant differences in both WT and Tg mice between the two exposures to a different number of cigarettes. Mice exposed to 6 cigarettes had higher leukocyte counts, with WT mice having a higher level of lymphocytes and Tg mice having higher levels of neutrophils, lymphocytes, and monocytes compared with mice exposed to 12 cigarettes. Likewise, both WT and Tg mice exposed to 6 cigarettes had higher levels of cholesterol, albumin, total protein, and ALT compared with mice exposed to 12 cigarettes. Measures of CACs also show that levels of these cells were not significantly different between WT and Tg mice exposed to the same number of cigarettes.

These data demonstrate increased systemic and hematological changes with a greater concentration of MCS exposure despite similar levels of urinary nicotine but do not seem to indicate that pulmonary overexpression of ecSOD is protective against injury related to MCS-exposure. Despite studies linking ROS with cigarettes and the development of CVD, it may be that ROS other than superoxide are responsible for this development. However, studies examining SOD in the role of smoking-related pulmonary disease have found that the overexpression of SOD can attenuate pulmonary inflammation and impaired lung function as well as the development of emphysema (477, 478), implicating superoxide in the development of pulmonary diseases related to the use of cigarettes. Other data demonstrate the role of ecSOD in the PM2.5-related impact on levels of CACs (299), and while PM is a known component of cigarette smoke (81), it may be that other constituents of MCS have a counteractive effect that, while potentially not injurious, is not easily visible in MCS as a complete mixture. Additionally, it could be that 
the adverse effects noted in response to MCS exposure are induced by systemic ROS, and thus the overexpression of ecSOD specifically in the lungs would not have an effect. It has been shown via measures of protein-acrolein adducts in the plasma and bone marrow that inhaled acrolein, a major constituent of MCS, is able to move into the bloodstream and exert its effects on CACs directly rather than indirectly by solely causing effects via the lungs (74). Although there is some endogenous production of acrolein and thus some naturally-occurring protein-acrolein adducts, the significantly increased levels of these adducts in acrolein-exposed mice indicates delivery of acrolein from the lungs to systemic sites where it can induce adverse effects (74). Based on these data, it may be systemic ROS is more important to cardiovascular-related outcomes than those found in the lungs; use of mice overexpressing ecSOD outside of the lungs or ecSOD knock-out mice may provide additional data regarding the role of ROS in the MCS-induced cardiovascular effects. Additionally, the use of ecSOD-Tg mice in chronic exposures and in exposures to specific constituents such as aldehydes could help clarify the role of superoxide in CVD and other diseases related to tobacco-product use and could help also identify which constituent(s) is responsible for specific outcomes.

The role of TRPA1 in the cardiovascular response to MCS and a constituent unsaturated aldehyde was also explored. Male TRPA1-null mice acutely exposed to MCS (4d; 12 cigarettes) showed minimal systemic changes compared with air-exposed control mice, the most significant being a decrease in leukocytes driven by decreases in lymphocytes and monocytes. However, this leukopenia was also noted in WT mice exposed to MCS under the same conditions, which would indicate that this effect is not due to activation of TRPA1. Likewise, the minimal systemic effects noted in both WT and 
TRPA1-null mice after MCS exposure would seem to indicate that the TRPA1 receptor is not serving a significant role in regard to these measures. Additionally, although MCSexposed WT mice showed a significant decrease in Sca-1+ cells, neither Sca-1+ cells nor CACs were measured in MCS-exposed TRPA1-null mice, so the impact of this receptor on these cells cannot be determined from the current study.

The lack of significant differences between WT and TRPA1-null mice exposed to MCS suggest that the outcomes measured in the current study are not mediated by TRPA1. However, other studies have clearly demonstrated the role of TRPA1 in MCS-induced diseases $(261,288,479,480)$, which may indicate that non-cardiovascular effects (e.g., pulmonary effects) are the most sensitive outcomes of MCS-exposure. The differing data may also indicate that our acute $(4 \mathrm{~d})$ exposure was not long enough to induce significant cardiovascular effects detectable in the measured outcomes or that $50 \%$ of the smoke from $12 \mathrm{KY}$ Reference 3R4F cigarettes was not sufficient to produce a significant cardiovascular-related effect.

To test whether the effects of CR, an unsaturated aldehyde found in high levels in MCS (156, 159), were also mediated by TRPA1, we exposed male TRPA1-null mice to CR (4d; 1 ppm). In contrast to the effects observed in CR-exposed male WT mice, TRPA1null mice had no systemic changes in $\mathrm{CBC}$, and plasma levels of the majority of the measured biomarkers were unchanged between the control and CR-exposed TRPA1-null mice. Cholesterol was decreased by CR exposure in the TRPA1-null mice (a change also observed in WT mice), although this change was due to decreased levels of LDL rather than by HDL as observed in WT mice. Furthermore, although it was only modestly significant, TRPA1-null male mice acutely exposed to CR showed an increase in CACs, 
whereas WT mice showed no change. Although some of the changes seen in WT and TRPA1-null mice in response to CR exposure are similar, the data overall—including data from other outcomes measured in CR-exposed WT and TRPA1-null mice $(481,482)$ seem to indicate that the vascular responses observed in male WT mice following CR exposure were mediated by TRPA1 activation, although chronic studies using TRPA1-null mice would serve to confirm this finding.

In summary, the studies presented in this chapter examined the roles of SOD and TRPA1 in the mechanism through which MCS and CR, an unsaturated aldehyde constituent, contribute to the development of CVD. Although previous studies have shown a role of superoxide and SOD in the development of smoking-related pulmonary diseases and in the mechanism through which $\mathrm{PM}_{2.5}$ decreases levels of CACs, the current study did not demonstrate a protective effect due to overexpression of pulmonary ecSOD in the systemic, hematological, and endothelium-related changes related to MCS. Chronic studies could help clarify whether protection conveyed by ecSOD occurs with longer exposure to MCS, while studies exposing ecSOD mice to $100 \%$ of the smoke generated from 6 or 12 cigarettes would help determine whether the lack of significant data was a result of levels of exposure too low to induce systemic or vascular changes. Additionally, exposure to specific constituent aldehydes could help identify whether individual components cause cardiovascular injury that may be masked by MCS as a complete mixture. Although the current study did not seem to suggest that the hematological, systemic, and CAC-related effects of MCS are produced through TRPA1, the current study of TRPA1-null mice exposed to CR does support the hypothesis that the cardiovascular effects of unsaturated aldehydes are mediated by TRPA1. Again, chronic studies of 
TRPA1-null mice exposed to MCS and to CR would further strengthen these findings.

Because of the commonality of exposure to MCS and unsaturated aldehydes from everyday sources and because of the risk of high-level aldehyde exposures related to accidents, chemical attacks, or occupational exposures, a better understanding of the mechanisms through which these compounds lead to CVD is necessary. These studies present data on the role of SOD in MCS-induced systemic injury and present novel research implicating TRPA1 in the mechanism of CR-induced toxicity, strengthening the hypothesis that $\mathrm{CR}$ works through a mechanism similar to acrolein to induce its cardiovascular effects. 
CHAPTER VIII

\section{SUMMARY \& CONCLUSIONS}

\section{A. Major Findings}

\section{Louisville Healthy Heart Study}

Circulating angiogenic cells (CACs) have been shown to be associated with endothelium health (62-66) as well as with well-known cardiovascular disease (CVD) risk factors $(66,70,305-308)$. CACs have also been associated with smoking, both with mainstream cigarette smoke (MCS) (315-319) and with individual MCS constituents, particularly acrolein $(71,74,151)$. Although the health effects of e-cigarettes (e-cigs) are still largely unknown, these shared constituents may contribute to adverse cardiovascular effects similar to those seen with the use of traditional cigarettes. Thus, the Louisville Healthy Heart Study was designed to examine the effect of tobacco product use (never smoker, former smoker, cigarette only use, and e-cig or dual use) on hematological measures, measures of urinary VOC and tobacco alkaloid metabolites, and levels of CACs. E-cig or dual users were significantly younger than other product use categories, an outcome similar to other studies that have found that e-cig use is most common among youth and young adults, but had significantly higher levels of leukocytes. Few studies have examined the effects of e-cig use on hematological outcomes, but the increased levels of 
leukocytes previously seen in smokers $(345,351-355)$ seem to indicate that there may be a similar mechanism inducing this increase. As this increase in smokers is typically attributed to inflammation $(345,354,356-358)$, additional measures of inflammatory markers in the LHHS cohort could help clarify this relationship. Analysis of urinary VOC metabolites showed significantly higher levels in users of tobacco products compared with nonsmokers. Although there were several metabolites that were significantly higher in cigarette only smokers compared with e-cig or dual users-supporting other studies showing that the production of VOCs in e-cigs may be less than that of traditional cigarettes - the levels measured in e-cig or dual users were still significantly higher than levels in both never and former smokers, indicating that the use of e-cigs alone or in combination with traditional cigarette retains the potential to induce cardiovascular effects through increased levels of VOCs. Analysis of CACs between the tobacco product use categories showed that only e-cig or dual users had levels of CACs that were significantly different than never smokers. CAC-3 (CD31+/CD34+/AC133+/CD45dim), an early endothelial progenitor cell population was significantly increased in e-cig or dual users, potentially indicative of increase endothelium repair initiated by a low but persistent level of injury induced by e-cig use. CAC-12 (CD45+), however, was significantly decreased with e-cig or dual use; more research is needed to clarify the role of the CD45 antigen in CACs.

Additional studies in a larger cohort and/or in a cohort with a greater number of ecig only users are needed to strengthen these findings. However, the similarity of outcomes seen between e-cig and dual users and users of traditional cigarettes alone seem to suggest that e-cig are capable of inducing hematological and endothelium-related effects that can 
subsequently contribute to the development of CVD. As e-cig alone and dual use becomes more prevalent among individuals of all age groups, it is imperative to gain a better understand of both the acute and chronic cardiovascular effects induced by these emerging tobacco devices.

\section{Constituent Aldehyde Exposures and Systemic and Hematological}

\section{Responses}

Tobacco smoking is the most significant modifiable risk factor in the development of $\operatorname{CVD}(1,14,75-77)$. Exposure to mainstream cigarette smoke (MCS) and individual aldehyde constituents including acrolein, crotonaldehyde (CR), formaldehyde (FR), and acetaldehyde (AA) have been linked to negative cardiovascular health outcomes $(1,91$ 94). The cardiovascular effects of emerging tobacco products including e-cigs are still largely unknown, although studies have detected aldehydes in e-cig aerosols $(96,180,207$ 213); the presence of these aldehydes may indicate the potential for negative cardiovascular effects similar to those seen with MCS. These studies focused on the systemic and hematological changes induced by inhalation exposure to MCS, e-cig aerosol, e-cig humectants, or constituent aldehydes (FR, AA, acrolein, and CR).

Many of our studies of mixtures, humectants, and individual aldehydes showed decreases in leukocytes after acute (4d or 2 weeks) exposure. Specifically, acute (4d) exposures to MCS, JUUL Mango and blu Plus+ aerosols, 30\%:70\% PG:VG, and acrolein and two weeks of exposure to formaldehyde $(5 \mathrm{ppm})$ caused significant decreases in leukocytes, while the remaining studies showed no significant changes between the airexposed control and the pollutant-exposed mice. Epidemiological studies of tobacco smokers have shown significant changes in hematological factors, particularly increased 
levels of leukocytes $(345,351-355)$, red blood cells $(345,414)$, and hemoglobin and platelets $(345,355,411-413)$. This contradiction may be related to the use of naïve mice, which may serve as a better model of health effects in new smokers rather than chronic smokers, or to our short exposure durations, which would not necessarily be comparable to effects seen in chronic smokers. Nonetheless, the significance of the changes seen in leukocytes reveal the potential of these mixtures and constituents to promote adverse hematological changes that could contribute to downstream disease development.

Systemically, mice exposed to CR (1 ppm) for 12 weeks showed significant increases in cholesterol and triglycerides; high levels of both of these biomarkers are known risk factors of ischemic (455) and coronary heart disease (456), and their increase in response to chronic $\mathrm{CR}(1 \mathrm{ppm})$ exposure may indicate that $\mathrm{CR}$ plays a role in these CVDs when MCS is a contributing factor. However, levels of these biomarkers did not increase with acute $(4 \mathrm{~d} ; 1 \mathrm{ppm})$ exposure, indicating that increases in lipids are not the primary change initiated by CR exposure.

Our studies of individual constituent aldehydes revealed that the concentration of the saturated aldehyde FR needed to induce significant systemic and hematological changes was much higher than the concentration required to see significant changes after exposure to unsaturated aldehydes ( 5 ppm versus $1 \mathrm{ppm})$. The carbon-carbon double bond in unsaturated aldehydes gives them a higher reactivity than saturated aldehydes, which may account for this difference. Acrolein has been shown to be a more potent irritant than FR (483), and odor detection and/or irritation of CR can occur at $<1 \mathrm{ppm}$ in approximately 1 minute (155). Chronic exposures to a low level (1 ppm) of FR or AA would allow for the examination of potential differences in cardiovascular toxicity related to saturated 
versus unsaturated aldehyde exposures.

\section{Aldehyde Exposures and Circulating Angiogenic Cells}

CACs are a population of stem cells that serve to repair endothelium dysfunction $(62,63)$ and that play an important role in overall endothelial function and health (63-66). Levels and function of CACs have been found to be inversely correlated with risk factors for atherosclerosis (70) as well as an individual's Framingham Risk Score (66). Inhalation exposure to acrolein has been shown to decrease levels of CACs in both humans (71) and mice $(74,151)$, but the effect of mixtures and other constituent aldehydes on CACs are still relatively unexplored. These studies focused on examining changes in CACs in response to inhalation exposures in mice as a marker of potential endothelium dysfunction. Data from these studies revealed that only female mice acutely (4d) exposed to JUUL Mango aerosol and male mice exposed to FR ( 2 weeks; 5 ppm) showed significant decreases in CACs. Measures of urinary metabolites in JUUL Mango-exposed male mice (6h) showed high levels of 3HPMA, the primary urinary metabolite of acrolein; as acrolein has been shown to decrease blood levels of CACs, it is likely that the decreased levels of CACs noted in JUUL Mango-exposed females is due to acrolein exposure. Additional studies in male mice exposed to JUUL Mango as well as examination of levels of Sca-1+ cells, the stem cell population from which CACs are derived, and levels of CACs in the bone marrow would help further clarify the mechanism through which JUUL Mango is inducing the significant decrease in CACs.

Although acute (4d) exposure to $5 \mathrm{ppm}$ FR increased levels of CACs, two weeks of exposure to the same concentration of FR significantly decreased CACs. The increase noted after four days may indicate a compensatory effect to repair damage within the 
endothelium, with the subsequent decrease a sign that this compensatory effect cannot be sustained. Urinary measures of formate in male mice exposed to FR alone (5 ppm, $6 \mathrm{~h}$ ) showed similar levels as male mice exposed to MCS (50\% smoke of $12 \mathrm{KY} 3 \mathrm{R} 4 \mathrm{~F}$ cigarettes, 6h). Taken with the outcomes from our two-week FR exposure in mice, these data may indicate that FR is contributing at least in part to the endothelium dysfunction noted in chronic smokers (85). However, in vitro studies exposing the aorta and the superior mesenteric artery to FR did not induce endothelial dysfunction (127), so the clinical relevance of these changes in CACs remains unclear. Likewise, although acrolein has been linked to endothelium dysfunction in mice (150), neither acute (4d) nor chronic (12 weeks) exposure to CR induced significant endothelium dysfunction $(481,482)$, nor were significant changes in CACs noted in either male ( 1 or 3 ppm, 4d; 1 ppm, 12 weeks) or female $(1 \mathrm{ppm}, 4 \mathrm{~d})$ mice with acute exposure. This difference may indicate that acrolein is more toxic to the endothelium than $\mathrm{CR}$, at least at the levels tested. Taken together, it appears that, at the concentrations and durations of exposure used in the current studies, CACs are likely not the earliest biomarker of harm affected in response to FR, AA, or CR exposure. While changes in the levels of these cells may play an important role in vascular and cardiovascular health, these data suggest that they should not be used as the only indicator of cardiovascular changes induced by aldehyde exposure. It would seem more appropriate that the level of CACs taken in conjunction with changes in systemic and hematological biomarkers as well as with measures of vascular function could denote exposure-related vascular changes that could indicate the potential for the development of CVD. Nonetheless, more studies in both male and female mice are needed to better understand the impact of specific constituent aldehydes on CACs and on other measures 
of endothelium dysfunction and to gain a better understanding of how these aldehydes contribute to MCS- and e-cig aerosol-induced CVD.

\section{Aldehyde Exposures and Sex Differences}

The data from our mixtures and individual aldehyde studies are largely consistent with data from a previous study showing that female mice exposed to a high level (250 ppm) of acrolein had reduced morbidity and mortality compared with similarly-exposed male mice (269). Our studies seem to demonstrate that females are protected against FRinduced systemic and hematological changes as well as changes in CACs. However, as most of the changes induced by FR in males occurred after two weeks, a two-week exposure in females at the same concentration $(5 \mathrm{ppm})$ would better clarify this dependence. Additionally, chronic studies in both males and females would provide an even greater understanding of this relationship. Likewise, a chronic exposure of female mice to CR (1 ppm, 12 weeks) would allow for a greater understanding of the sexdependence noted between male and female mice after acute (4d) exposure to CR (1 ppm). Although male mice acutely exposed to CR showed minimal but significant systemic and hematological changes as well as a significant increase in Sca-1+ cells (1 ppm), females showed no significant changes in any of the measured biomarkers. These differences seem to support the hypothesis that females are somewhat protected against toxicity and injury induced by unsaturated aldehydes.

Several epidemiological studies seem to show outcomes consistent with this hypothesis as well, particularly showing that females are less sensitive to tobacco-induced atherosclerotic damage than males (484); this difference is likely due to the protective effects of female hormones such as estrogen (484). The hormone prolactin may also play 
a significant role in the sex-dependent role of these aldehydes. Female mice have been shown to have a higher sensitivity to pain-inducing stimuli than males, driven largely by levels of prolactin (485). Prolactin has been shown to regulate expression of the TRPA1 receptor, which serves as a pain and irritant receptor in sensory neurons (486), and Conklin et al. (269) have proposed that perhaps females have a greater expression of TRPA1 that promotes a quicker response to irritants such as unsaturated aldehydes. In fact, females exposed to high-level acrolein $(250 \mathrm{ppm})$ showed a quicker "respiratory braking" response and decrease their breathing rate quicker than male mice under the same exposure conditions (269). This variance in the change in breathing rate is not seen in male and female TRPA1-null mice, further suggesting that the female protection is related to TRPA1 (277). Additional studies are needed to determine the exact mechanism of this sexdependent protection in response to exposure to unsaturated aldehydes as well as to mixtures such as MCS and e-cig aerosol.

\section{Mainstream Cigarette Smoke and Superoxide Dismutase}

The superoxide dismutase (SOD) enzyme indirectly plays a role in maintaining proper endothelium function by catalyzing the dismutation of superoxide. If left as a free molecule in the blood, superoxide can bind with nitric oxide (NO) and decrease the bioavailability of NO $(40,41,49,50,53)$; the resulting decrease of this vasodilator can allow for the development of endothelium dysfunction $(40,41)$, which has been linked to a number of CVDs (54-58). SOD has also been linked to the development of cigarette smoke-induced pulmonary diseases $(477,478)$, and the administration of SOD to serve as an antioxidant defense against the loss of $\mathrm{NO}$ - and the subsequent development of endothelium dysfunction and, perhaps, disease — has been proposed (53, 487, 488). 
The results of our studies in mice overexpressing pulmonary extracellular SOD (ecSOD-Tg) after exposure to MCS (50\% of smoke of 6 or $12 \mathrm{KY}$ Reference 3R4F cigarettes), however, do not seem to support this proposed medical intervention. Although there were differences between WT and ecSOD-Tg mice exposed to cigarettes compared with baseline, there were no significant differences between WT and ecSOD-Tg mice exposed to the same number of cigarettes. Measures of urinary metabolites did not show significant differences in the excretion of unsaturated aldehyde metabolites or total urinary nicotine metabolites between the two strains of mice, and measures of systemic and hematological biomarkers showed few if any significant differences between WT and ecSOD-Tg mice at the same level of exposure. Likewise, CAC levels were not significantly different between the two strains after exposure to either 6 or 12 cigarettes. Chronic studies would help elucidate whether any protective effect of SOD exists but takes longer than four days to become apparent in the measured outcomes. Additionally, exposures of ecSOD-Tg mice to individual constituent aldehydes could help explore the role of SOD in the mechanism of specific aldehydes.

\section{Aldehydes and the Transient Receptor Potential Ankyrin 1 Channel}

The transient receptor potential ankyrin 1 (TRPA1) channel is activated by a variety of air pollutants, including MCS and aldehydes (127, 260, 262, 266, 268-270). Although TRPA1 is primarily recognized as a pain receptor $(262,272,279)$, it also play an important role in the irritant response induced by inhaled toxins $(262,264,279,286)$, including respiratory braking (slowing rate and depth of breathing), which serves to reduce inhalation and thus exposure to the toxin $(261,489)$. This function is supported by the delayed onset of respiratory braking and the higher incidence of morbidity and mortality seen in TRPA1- 
null mice exposed to a high level of acrolein compared with similarly-exposed WT mice (269). The limited changes induced by CR in TRPA1-null mice compared with CRexposed WT mice seem to indicate that the systemic and hematological changes noted in male WT mice following CR exposure were mediated by TRPA1. Chronic studies using TRPA1-null mice would serve to strengthen this finding, while a chronic study exposing TRPA1-null mice to acrolein under the same conditions (1 ppm; 12 weeks) would further confirm the hypothesis that unsaturated aldehydes induce CVD effects through the TRPA1 channel. Additionally, exposures of TRPA1-null mice to FR and AA would allow for the determination as to whether saturated aldehydes induce CVD effects through the same pathway. Studies have shown that the pain responses induced by FR and AA are mediated through TRPA1 $(490,491)$ as is the FR-induced-but not the AA-induced-relaxation of the superior mesenteric artery $(127,492)$, but the mechanism through which these aldehydes induce cardiovascular effects has not been determined.

\section{B. Strengths}

There are several strengths of the human study presented in this dissertation. First, although the Louisville Healthy Heart cohort was not as large as national epidemiological cohorts, we were still able to examine tobacco product use, urinary VOC metabolites, and CACs in a population of up to 143 individuals. Many previous investigations of CVD and CACs $(66,70,307,309,493)$ as well as several studies that have looked at CACs in relation to smoking $(315,316,318)$ have utilized cohorts of less than 100 people, while only a few studies $(311,494,495)$, including the predecessor to our study (71) have examined these relationships in larger cohorts. Additionally, two-thirds of our participants report a diagnosis of some form of CVD, and as the prevalence of CVD continues to grow 
worldwide, it becomes increasingly important to perform research in susceptible individuals to provide accurate data that can be used to inform the development of better cardiovascular health care practices. Our study is also one of the first to look at the relationship between CACs and the use of ENDS devices, especially in fifteen antigenically distinct CAC populations. Looking at the association between these populations, product use, and CVD allowed us to examine changes in specific cell types and subpopulations that may be linked to specific risk factors, as had previously been shown (73).

There are also a number of strengths in our murine exposure studies. The results of our urinary metabolite studies demonstrate that the levels of exposure used in our individual constituent aldehyde studies are comparable to real-world exposures, which validates our outcomes as cardiovascular and systemic effects that could be seen in humans in response to aldehyde exposures. Our studies of CR are the first to show the systemic, hematological, and endothelium-related effects of $\mathrm{CR}$ exposure in vivo and to further show that these effects are both sex- and TRPA1-dependent, the latter of which may present a therapeutic target for treatment of aldehyde exposures. Our studies of FR and AA are also some of the first to look at cardiovascular effects in response to acute exposures and again to suggest sex-dependent differences in outcomes.

\section{Limitations}

Despite the many strengths of our studies, there are also a number of limitations. Despite the size of the Louisville Healthy Heart cohort compared with other cohorts examining CACs, the study is still relatively small, and it would therefore be unwise to extrapolate the findings to the population at large, although the outcomes do present interesting and specific effects that ought to be examined in a larger cohort. Likewise, the 
number of e-cig only users and dual users was small. Other cohorts report similar limitations in recruiting e-cig-only users (395), and large epidemiological studies have reported that e-cig users are likely to be dual users $(496,497)$, making recruitment of e-cig only users more difficult. However, if dual use is the most common form of e-cig use, having a dual use group in future cohort studies would allow for analysis of outcomes in a group most relevant to real-world e-cig use. Of the e-cig alone and dual users in our study, all reported use of 1 st and 2 nd generation e-cigs (cigalikes and vape pens, respectively), and thus our findings cannot be generalized to users of 3 rd (box mod) and 4th (vape pod) generation e-cig devices or to more recent products such as puff pods and puff bars. The self-report nature of our questionnaire also presents a limitation, especially in regarding to self-reported smoking status. Although questions related to tobacco use or exposure to secondhand smoke were carefully structured to avoid misclassification, we can only assume the individuals were truthful in their responses. We can assume some level of confidence in self-reported smoking status based on measured of urinary cotinine, but nonsmoking individuals who are exposed to high levels of secondhand smoke could have relatively high levels of urinary cotinine, which could confound the outcomes.

In regard to other measures that could play an important role in the development of CVD, although we have measures of body mass index (BMI) for the participants, we do not have measures of visceral adiposity or wait-to-hip ratios. These alternate measures have been proposed as comparable if not better indicators of obesity than BMI (498), and several epidemiological studies have found significant associations between waist-to-hip ratios and cardiovascular events and/or cardiovascular-related death (499-501). As obesity is a known risk factor for $\operatorname{CVD}(502,503)$, future human studies examining CVD should 
make obtaining these measures part of their protocol in order to provide a more comprehensive assessment of a participant's CVD risk.

There are also limitations in our murine studies. First, mice are "involuntary smokers," and they therefore decrease the rate and depth of their breathing in response to exposure to noxious compounds such as aldehydes (269); this reflex is known as the "respiratory braking response" (489). Second, estimations of actual aldehyde exposure in mice was measured by urinary metabolites. Because aldehydes metabolize quickly (99, $100,108,109,504)$, it is difficult to measure levels of these compounds in the blood, but levels of urinary metabolites have been shown to be proportional to and thus useful biomarkers of aldehyde exposure $(147,159)$. Nonetheless, even at relevant exposure concentrations, our studies did not always show significant systemic or hematological changes. This could be because the level of exposure was too low and/or the duration of the exposure was not sufficient to cause significant cardiovascular outcomes. The duration of our exposure is also a limitation in that the time frame during which mice are exposed (6h/day) may be more concentrated than that of a typical tobacco product user. That is, although the puff protocol used in our MCS and e-cig exposures are designed to mimic the episodic nature of tobacco product use (smoking or vaping for a brief session followed by a period of non-use before smoking or vaping again), tobacco product use in humans does not occur only within a six hour window in any given day; the longer window of exposure would likely allow for variations in metabolism and clearance greater than that seen in our urinary metabolite data and, thus, likely allow for a lower level of exposure to harmful constituents. Therefore, although the effects induced in our exposed mice may indicate effects that could occur in tobacco product users, these outcomes may take longer to 
manifest in humans compared with mice when accounting for differences in daily exposure durations. The aldehydes used in our studies are known to be carcinogenic, and it is possible that cancer would develop before the development of negative cardiovascular effects at the tested concentrations and durations. Additionally, CACs as a marker of vascular injury were not always measured due to limited blood available for analyses, so it is possible changes in CACs that could lead to subsequent CVD development were induced but not noted. Similarly, we did not always perform the same exposures in both male and female mice, so we cannot necessarily speak to the sex-dependence of the measured outcomes when there was not a directly comparable study.

\section{Future Directions}

More research is still needed to explore the toxicity of traditional cigarettes and ENDS in both human cohorts and murine models. Additional studies with focused recruitment of e-cig only users are needed in order to better understand the health effects attributable to e-cig use rather than effects potentially compounded from the use of both ecigs and traditional cigarettes. These cohorts should also work to recruit users of more recent generations of e-cig devices (mechanical mods, variable voltage devices, JUUL and other pod-based devices) and other ENDS to better understand differences in health outcomes related to different ENDS, especially as more recent generations of these devices

continue to grow in popularity among adolescents. In regard to research using murine models, future studies could increase the concentration of the aldehydes used for exposure —although concentrations should be compared with those found in mixtures to ensure their real-world relevance - or the length of the exposure to test whether chronic exposures at varying doses, as would be relevant in addicted tobacco-product users, produce additional 
systemic and vascular changes.

Furthermore, despite looking at a number of different aldehydes, the actual constituent responsible for the toxicity of smoking has not been identified, so additional studies of different shared constituents as well as additional e-cig flavorings could help with this identification. Combination studies should also be performed using individual aldehyde constituents and nicotine. As users of tobacco products are exposed to aldehydes and nicotine simultaneously, and as nicotine alone has been shown to induce cardiovascular effects, a better understanding of how aldehydes and nicotine in combination affect our cardiovascular outcomes of interest is required, particularly if exposure to these compounds in combination could potentially induce outcomes varying from those seen with individual exposures. Finally, as we used whole-body TRPA1-null mice in our MCS and unsaturated aldehyde studies, we cannot rule out a role of extra-pulmonary (neuronal) TRPA1 in these outcomes. In fact, TRPA1 receptors are widely distributed in nonneuronal sites, such as the urothelium, the heart, and the vasculature including the endothelium $(127,275-277,280)$. The exact location of the TRPA1 that is activated by inhaled MCS and MCS-related toxicants (and responsible for systemic effects) will need to be established in more specific models of TRPA1 deletion.

\section{E. Conclusions}

Overall, the work described here demonstrates the adverse systemic, hematological, and endothelium-related effects induced by exposure to traditional cigarettes and e-cigs and supports the hypothesis that much of the cardiovascular injury related to the use of these products is related to aldehydes, particularly the unsaturated aldehydes, through the TRPA1 channel. 
Our human studies showed that e-cig or dual users were younger than other tobacco product use categories, as has been reported in previous cohort studies. Relatedly, the ecig or dual users in our study had significantly less prevalence of CVD and of CVD-related medication usage. E-cig or dual users also showed significantly increased levels of leukocytes - perhaps indicative of increased inflammation as seen in users of traditional cigarettes - as well as increased levels of CAC-3 (CD31+/CD34+/AC133+/CD45dim), which may be related to a persistent, low level of injury induced by some unique component of e-cigs or of combined e-cig and cigarette use. Conversely, CAC-12 (CD45+) was significantly decreased in this group of users, but further study is needed to better understand this change. Although e-cig or dual users had levels of some urinary VOC metabolites that were significantly lower than levels in users of traditional cigarettes alone, almost all measured VOC metabolite levels were still significantly higher in e-cig or dual users compared with both never and former smokers. These data confirm that while e-cigs may be less harmful than traditional cigarettes in regard to VOC exposures, they still produce VOCs at levels capable of inducing adverse cardiovascular effects.

Our murine studies indicate that short-term exposures to e-cig aerosols and PG:VG produce hematological changes similar to those seen with MCS exposure, as does two weeks of exposure to FR. Similarly, chronic CR exposure shows dyslipidemic changes that could contribute to the development of CVD. Short-term exposures to AA, however, produced limited systemic and hematological changes. Only short-term exposure to JUUL Mango in females and two weeks of exposure to FR (5 ppm) causes significant decreases in CACs, indicating that endothelium dysfunction is likely not one of the early changes caused by exposure to these mixtures and individual aldehydes, at least not at the 
concentrations and durations tested. The specificity of the change in CACs does also indicate the need for additional studies of specific e-cig flavors, as different flavors may cause different cardiovascular outcomes, and for the continued study of individual aldehyde constituents. Additionally, these studies show the importance of the TRPA1 receptor in the systemic and hematological changes induced by CR exposure as well as the sex-dependent nature of CR and, potentially, FR and e-cig aerosols.

Future studies are required to better understand the role of tobacco products, particularly e-cigs, in the development of CVD and to continue working towards a better understanding of the mechanisms through which aldehydes contribute to the burden of tobacco-related CVD. 


\section{REFERENCES}

1. Benjamin, E.J., Muntner, P., Alonso, A., Bittencourt, M.S., Callaway, C.W., Carson, A.P., Chamberlain, A.M., Chang, A.R., Cheng, S., Das, S.R., Delling, F.N., Djousse, L., Elkind, M.S.V., Ferguson, J.F., Fornage, M., Jordan, L.C., Khan, S.S., Kissela, B.M., Knutson, K.L., Kwan, T.W., Lackland, D.T., Lewis, T.T., Lichtman, J.H., Longenecker, C.T., Loop, M.S., Lutsey, P.L., Martin, S.S., Matsushita, K., Moran, A.E., Mussolino, M.E., O'Flaherty, M., Pandey, A., Perak, A.M., Rosamond, W.D., Roth, G.A., Sampson, U.K.A., Satou, G.M., Schroeder, E.B., Shah, S.H., Spartano, N.L., Stokes, A., Tirschwell, D.L., Tsao, C.W., Turakhia, M.P., VanWagner, L.B., Wilkins, J.T., Wong, S.S. and Virani, S.S. (2019). Heart Disease and Stroke Statistics-2019 Update: A Report From the American Heart Association. Circulation 139: e56-e528.

2. The top 10 causes of death. 2018. Available from: http://www.who.int/newsroom/fact-sheets/detail/the-top-10-causes-of-death. Accessed June 152018.

3. Fryar, C.D., Chen, T.C. and Li, X. (2012). Prevalence of uncontrolled risk factors for cardiovascular disease: United States, 1999-2010. NCHS data brief: 1-8.

4. $\quad$ Nawrot, T.S., Perez, L., Künzli, N., Munters, E. and Nemery, B. (2011). Public health importance of triggers of myocardial infarction: a comparative risk assessment. The Lancet 377: 732-740.

5. Dockery, D.W., Pope, C.A., Xu, X., Spengler, J.D., Ware, J.H., Fay, M.E., Ferris, B.G. and Speizer, F.E. (1993). An Association between Air Pollution and Mortality in Six U.S. Cities. New Engl J Med 329: 1753-1759.

6. Laden, F., Schwartz, J., Speizer, F.E. and Dockery, D.W. (2006). Reduction in fine particulate air pollution and mortality: Extended follow-up of the Harvard Six Cities study. American journal of respiratory and critical care medicine 173: 667-672.

7. Lepeule, J., Laden, F., Dockery, D. and Schwartz, J. (2012). Chronic exposure to fine particles and mortality: an extended follow-up of the Harvard Six Cities study from 1974 to 2009. Environ Health Perspect 120: 965-970.

8. Puett, R.C., Hart, J.E., Yanosky, J.D., Paciorek, C., Schwartz, J., Suh, H., Speizer, F.E. and Laden, F. (2009). Chronic Fine and Coarse Particulate Exposure, Mortality, and Coronary Heart Disease in the Nurses' Health Study. Environ Health Persp 117: 16971701.

9. Pope, C.A., Burnett, R.T., Thurston, G.D., Thun, M.J., Calle, E.E., Krewski, D. and Godleski, J.J. (2004). Cardiovascular mortality and long-term exposure to particulate 
air pollution: epidemiological evidence of general pathophysiological pathways of disease. Circulation 109: 71-77.

10. Van Hee, V.C., Adar, S.D., Szpiro, A.A., Barr, R.G., Bluemke, D.A., Diez Roux, A.V., Gill, E.A., Sheppard, L. and Kaufman, J.D. (2009). Exposure to traffic and left ventricular mass and function: the Multi-Ethnic Study of Atherosclerosis. American journal of respiratory and critical care medicine 179: 827-834.

11. Auchincloss, A.H., Diez Roux, A.V., Dvonch, J.T., Brown, P.L., Barr, R.G., Daviglus, M.L., Goff, D.C., Kaufman, J.D. and O'Neill, M.S. (2008). Associations between recent exposure to ambient fine particulate matter and blood pressure in the Multi-ethnic Study of Atherosclerosis (MESA). Environ Health Perspect 116: 486-491.

12. Adar, S.D., Sheppard, L., Vedal, S., Polak, J.F., Sampson, P.D., Diez Roux, A.V., Budoff, M., Jacobs, D.R., Jr., Barr, R.G., Watson, K. and Kaufman, J.D. (2013). Fine particulate air pollution and the progression of carotid intima-medial thickness: a prospective cohort study from the multi-ethnic study of atherosclerosis and air pollution. PLoS medicine 10: e1001430.

13. Wilker, E.H., Mittleman, M.A., Coull, B.A., Gryparis, A., Bots, M.L., Schwartz, J. and Sparrow, D. (2013). Long-term Exposure to Black Carbon and Carotid IntimaMedia Thickness: The Normative Aging Study. Environ Health Persp 121: 1061-1067.

14. Bhatnagar, A. (2006). Environmental cardiology: studying mechanistic links between pollution and heart disease. Circ Res 99: 692-705.

15. Brook, R.D., Franklin, B., Cascio, W., Hong, Y., Howard, G., Lipsett, M., Luepker, R., Mittleman, M., Samet, J., Smith, S.C., Jr., Tager, I., Expert Panel on, P. and Prevention Science of the American Heart, A. (2004). Air pollution and cardiovascular disease: a statement for healthcare professionals from the Expert Panel on Population and Prevention Science of the American Heart Association. Circulation 109: 2655-2671.

16. Brook, R.D., Rajagopalan, S., Pope, C.A., Brook, J.R., Bhatnagar, A., Diez-Roux, A.V., Holguin, F., Hong, Y., Luepker, R.V., Mittleman, M.A., Peters, A., Siscovick, D., Smith, S.C., Jr., Whitsel, L., Kaufman, J.D., American Heart Association Council on, E., Prevention, C.o.t.K.i.C.D., Council on Nutrition, P.A. and Metabolism. (2010). Particulate matter air pollution and cardiovascular disease: An update to the scientific statement from the American Heart Association. Circulation 121: 2331-2378.

17. Pope, C.A. and Dockery, D.W. (2006). Health Effects of Fine Particulate Air Pollution: Lines that Connect. J Air Waste Manage Assoc 56: 709-742.

18. Shah, A.S., Langrish, J.P., Nair, H., McAllister, D.A., Hunter, A.L., Donaldson, K., Newby, D.E. and Mills, N.L. (2013). Global association of air pollution and heart failure: a systematic review and meta-analysis. Lancet 382: 1039-1048.

19. Dvonch, J.T., Kannan, S., Schulz, A.J., Keeler, G.J., Mentz, G., House, J., Benjamin, A., Max, P., Bard, R.L. and Brook, R.D. (2009). Acute effects of ambient 
particulate matter on blood pressure: differential effects across urban communities. Hypertension 53: 853-859.

20. Ibald-Mulli, A., Stieber, J., Wichmann, H., Koenig, W. and Peters, A. (2001). Effects of air pollution on blood pressure: a population-based approach. American Journal of Public Health 91: 571-577.

21. Langrish, J.P., Li, X., Wang, S., Lee, M.M.Y., Barnes, G.D., Miller, M.R., Cassee, F.R., Boon, N.A., Donaldson, K., Li, J., Li, L., Mills, N.L., Newby, D.E. and Jiang, L. (2012). Reducing Personal Exposure to Particulate Air Pollution Improves Cardiovascular Health in Patients with Coronary Heart Disease. Environ Health Persp 120: $367-372$.

22. Peters, A., Dockery, D.W., Muller, J.E. and Mittleman, M.A. (2001). Increased Particulate Air Pollution and the Triggering of Myocardial Infarction. Circulation 103: 2810-2815.

23. Künzli, N., Jerrett, M., Garcia-Esteban, R., Basagaña, X., Beckermann, B., Gilliland, F., Medina, M., Peters, J., Hodis, H.N. and Mack, W.J. (2010). Ambient Air Pollution and the Progression of Atherosclerosis in Adults. PloS one 5: 1-10.

24. Lundback, M., Mills, N.L., Lucking, A., Barath, S., Donaldson, K., Newby, D.E., Sandstrom, T. and Blomberg, A. (2009). Experimental exposure to diesel exhaust increases arterial stiffness in man. Particle and fibre toxicology 6: 7.

25. Cosselman, K.E., Krishnan, R.M., Oron, A.P., Jansen, K., Peretz, A., Sullivan, J.H., Larson, T.V. and Kaufman, J.D. (2012). Blood pressure response to controlled diesel exhaust exposure in human subjects. Hypertension 59: 943-948.

26. Medina-Ramon, M., Goldberg, R., Melly, S., Mittleman, M.A. and Schwartz, J. (2008). Residential exposure to traffic-related air pollution and survival after heart failure. Environ Health Perspect 116: 481-485.

27. Brook, R.D. and Rajagopalan, S. (2009). Particulate matter, air pollution, and blood pressure. Journal of the American Society of Hypertension : JASH 3: 332-350.

28. McDonald, J.D., Reed, M.D., Campen, M.J., Barrett, E.G., Seagrave, J. and Mauderly, J.L. (2007). Health effects of inhaled gasoline engine emissions. Inhalation toxicology 19 Suppl 1: 107-116.

29. Meng, Q., Richmond-Bryant, J., Lu, S.-E., Buckley, B., Welsh, W.J., Whitsel, E.A., Hanna, A., Yeatts, K.B., Warren, J., Herring, A.H. and Xiu, A. (2013). Cardiovascular Outcomes and the Physical and Chemical Properties of Metal Ions Found in Particulate Matter Air Pollution: A QICAR Study. Environ Health Persp 121: 558-564.

30. Peters, A. (2009). Air quality and cardiovascular health: smoke and pollution matter. Circulation 120: 924-927. 
31. Sun, Q., Hong, X. and Wold, L.E. (2010). Cardiovascular effects of ambient particulate air pollution exposure. Circulation 121: 2755-2765.

32. Uzoigwe, J.C., Prum, T., Bresnahan, E. and Garelnabi, M. (2013). The Emerging Role of Outdoor and Indoor Air Pollution in Cardiovascular Disease. North American Journal of Medical Sciences 5: 445-453.

33. Lerman, A. and Zeiher, A.M. (2005). Endothelial Function: Cardiac Events. Circulation 111: 363-368.

34. Calderon-Garciduenas, L., Franco-Lira, M., Torres-Jardon, R., Henriquez-Roldan, C., Barragan-Mejia, G., Valencia-Salazar, G., Gonzalez-Maciel, A., Reynoso-Robles, R., Villarreal-Calderon, R. and Reed, W. (2007). Pediatric respiratory and systemic effects of chronic air pollution exposure: nose, lung, heart, and brain pathology. Toxicol Pathol 35: 154-162.

35. Cellini, M., Strobbe, E., Gizzi, C., Balducci, N., Toschi, P.G. and Campos, E.C. (2012). Endothelin-1 plasma levels and vascular endothelial dysfunction in primary open angle glaucoma. Life sciences 91: 699-702.

36. Leppert, J., Ringqvist, A., Karlberg, B.E. and Ringqvist, I. (1998). Whole-body cooling increases plasma endothelin-1 levels in women with primary Raynaud's phenomenon. Clinical physiology (Oxford, England) 18: 420-425.

37. O'Neill, M.S., Veves, A., Zanobetti, A., Sarnat, J.A., Gold, D.R., Economides, P.A., Horton, E.S. and Schwartz, J. (2005). Diabetes enhances vulnerability to particulate air pollution-associated impairment in vascular reactivity and endothelial function. Circulation 111: 2913-2920.

38. Spiropoulos, K., Trakada, G., Nikolaou, E., Prodromakis, E., Efremidis, G., Pouli, A. and Koniavitou, A. (2003). Endothelin-1 levels in the pathophysiology of chronic obstructive pulmonary disease and bronchial asthma. Respiratory Medicine 97: 983-989.

39. Toda, N. and Okamura, T. (2013). Obesity impairs vasodilatation and blood flow increase mediated by endothelial nitric oxide: An overview. Journal of Clinical Pharmacology 53: 1228-1239.

40. Forstermann, U. (2008). Oxidative stress in vascular disease: causes, defense mechanisms and potential therapies. Nature clinical practice. Cardiovascular medicine 5: 338-349.

41. Li, H., Horke, S. and Forstermann, U. (2013). Oxidative stress in vascular disease and its pharmacological prevention. Trends in pharmacological sciences 34: 313-319.

42. Zuo, L., Zhou, T., Pannell, B.K., Ziegler, A.C. and Best, T.M. (2015). Biological and physiological role of reactive oxygen species--the good, the bad and the ugly. Acta physiologica (Oxford, England) 214: 329-348. 
43. Bayir, H. (2005). Reactive oxygen species. Critical care medicine 33: S498-501.

44. Mittler, R. (2017). ROS Are Good. Trends in Plant Science 22: 11-19.

45. Kaehler, J., Sill, B., Koester, R., Mittmann, C., Orzechowski, H.D., Muenzel, T. and Meinertz, T. (2002). Endothelin-1 mRNA and protein in vascular wall cells is increased by reactive oxygen species. Clinical science (London, England : 1979) 103 Suppl 48: 176s-178s.

46. Yang, W. and Omaye, S.T. (2009). Air pollutants, oxidative stress and human health. Mutation Research/Genetic Toxicology and Environmental Mutagenesis 674: 4554.

47. Betteridge, D.J. (2000). What is oxidative stress? Metabolism 49: 3-8.

48. Brieger, K., Schiavone, S., Miller, F.J., Jr. and Krause, K.H. (2012). Reactive oxygen species: from health to disease. Swiss medical weekly 142: w13659.

49. Feletou, M. and Vanhoutte, P.M. (2006). Endothelial dysfunction: a multifaceted disorder (The Wiggers Award Lecture). American journal of physiology. Heart and circulatory physiology 291: H985-1002.

50. Langrish, J.P., Bosson, J., Unosson, J., Muala, A., Newby, D.E., Mills, N.L., Blomberg, A. and Sandström, T. (2012). Cardiovascular effects of particulate air pollution exposure: time course and underlying mechanisms. Journal of internal medicine 272: 224-239.

51. Nelson, M.-A.M., Baba, S.P. and Andersonc, E.J. (2017). Biogenic Aldehydes as Therapeutic Targets for Cardiovascular Disease. Current opinion in pharmacology 33: 56-63.

52. Utell, M.J., Frampton, M.W., Zareba, W., Devlin, R.B. and Cascio, W.E. (2002). CARDIOVASCULAR EFFECTS ASSOCIATED WITH AIR POLLUTION: POTENTIAL MECHANISMS AND METHODS OF TESTING. Inhalation toxicology 14: 1231-1247.

53. Fukai, T. and Ushio-Fukai, M. (2011). Superoxide dismutases: role in redox signaling, vascular function, and diseases. Antioxidants \& redox signaling 15: 15831606.

54. Davignon, J. and Ganz, P. (2004). Role of Endothelial Dysfunction in Atherosclerosis. Circulation 109: III-27-III-32.

55. Mudau, M., Genis, A., Lochner, A. and Strijdom, H. (2012). Endothelial dysfunction: the early predictor of atherosclerosis. Cardiovascular Journal of Africa 23: 222-231. 
56. Owens, G.K., Kumar, M.S. and Wamhoff, B.R. (2004). Molecular regulation of vascular smooth muscle cell differentiation in development and disease. Physiological reviews 84: 767-801.

57. Rossi, R., Chiurlia, E., Nuzzo, A., Cioni, E., Origliani, G. and Modena, M.G. (2004). Flow-mediated vasodilation and the risk of developing hypertension in healthy postmenopausal women. Journal of the American College of Cardiology 44: 1636-1640.

58. Budhiraja, R., Tuder, R.M. and Hassoun, P.M. (2004). Endothelial Dysfunction in Pulmonary Hypertension. Circulation 109: 159-165.

59. Bielli, A., Scioli, M.G., Mazzaglia, D., Doldo, E. and Orlandi, A. (2015). Antioxidants and vascular health. Life sciences 143: 209-216.

60. Cervantes Gracia, K., Llanas-Cornejo, D. and Husi, H. (2017). CVD and Oxidative Stress. Journal of Clinical Medicine 6: 22.

61. Panth, N., Paudel, K.R. and Parajuli, K. (2016). Reactive Oxygen Species: A Key Hallmark of Cardiovascular Disease. Advances in Medicine 2016: 12.

62. Asahara, T., Masuda, H., Takahashi, T., Kalka, C., Pastore, C., Silver, M., Kearne, M., Magner, M. and Isner, J.M. (1999). Bone Marrow Origin of Endothelial Progenitor Cells Responsible for Postnatal Vasculogenesis in Physiological and Pathological Neovascularization. Circ Res 85: 221-228.

63. Kolluru, G.K., Bir, S.C. and Kevil, C.G. (2012). Endothelial Dysfunction and Diabetes: Effects on Angiogenesis, Vascular Remodeling, and Wound Healing. International Journal of Vascular Medicine 2012: 30.

64. Deanfield, J.E., Halcox, J.P. and Rabelink, T.J. (2007). Endothelial Function and Dysfunction: Testing and Clinical Relevance. Circulation 115: 1285-1295.

65. Diehl, K.J., Weil, B.R., Westby, C.M., MacEneaney, O.J., Kushner, E.J., Greiner, J.J. and DeSouza, C.A. (2012). Effects of endothelin-1 on endothelial progenitor cell function. Clinical Chemistry \& Laboratory Medicine 50: 1121-1124.

66. Hill, J.M., Zalos, G., Halcox, J.P.J., Schenke, W.H., Waclawiw, M.A., Quyyumi, A.A. and Finkel, T. (2003). Circulating Endothelial Progenitor Cells, Vascular Function, and Cardiovascular Risk. The New England journal of medicine 348: 593-600.

67. Haberzettl, P., Lee, J., Duggineni, D., McCracken, J., Bolanowski, D., O'Toole, T.E., Bhatnagar, A. and Conklin, D.J. (2012). Exposure to Ambient Air Fine Particulate Matter Prevents VEGF-Induced Mobilization of Endothelial Progenitor Cells from the Bone Marrow. Environ Health Perspect 120: 848-856.

68. Paczkowska, E., Gołąb-Janowska, M., Bajer-Czajkowska, A., Machalińska, A., Ustianowski, P., Rybicka, M., Kłos, P., Dziedziejko, V., Safranow, K., Nowacki, P. and 
Machaliński, B. (2013). Increased circulating endothelial progenitor cells in patients with haemorrhagic and ischaemic stroke: The role of Endothelin-1. J Neurol Sci 325: 90-99.

69. Shantsila, E., Watson, T. and Lip, G.Y.H. (2007). Endothelial Progenitor Cells in Cardiovascular Disorders. Journal of the American College of Cardiology 49: 741-752.

70. Vasa, M., Fichtlscherer, S., Aicher, A., Adler, K., Urbich, C., Martin, H., Zeiher, A.M. and Dimmeler, S. (2001). Number and Migratory Activity of Circulating Endothelial Progenitor Cells Inversely Correlate With Risk Factors for Coronary Artery Disease. Circ Res 89: e1-e7.

71. DeJarnett, N., Conklin, D.J., Riggs, D.W., Myers, J.A., O'Toole, T.E., Hamzeh, I., Wagner, S., Chugh, A., Ramos, K.S., Srivastava, S., Higdon, D., Tollerud, D.J., DeFilippis, A., Becher, C., Wyatt, B., McCracken, J., Abplanalp, W., Rai, S.N., Ciszewski, T., Xie, Z., Yeager, R., Prabhu, S.D. and Bhatnagar, A. (2014). Acrolein exposure is associated with increased cardiovascular disease risk. Journal of the American Heart Association 3: e000934.

72. Heiss, C., Amabile, N., Lee, A.C., Real, W.M., Schick, S.F., Lao, D., Wong, M.L., Jahn, S., Angeli, F.S., Minasi, P., Springer, M.L., Hammond, S.K., Glantz, S.A., Grossman, W., Balmes, J.R. and Yeghiazarians, Y. (2008). Brief Secondhand Smoke Exposure Depresses Endothelial Progenitor Cells Activity and Endothelial Function: Sustained Vascular Injury and Blunted Nitric Oxide Production. Journal of the American College of Cardiology 51: 1760-1771.

73. O'Toole, T.E., Hellmann, J., Wheat, L., Haberzettl, P., Lee, J., Conklin, D.J., Bhatnagar, A. and Pope, C.A., 3rd. (2010). Episodic exposure to fine particulate air pollution decreases circulating levels of endothelial progenitor cells. Circ Res 107: 200203.

74. Wheat, L.A., Haberzettl, P., Hellmann, J., Baba, S.P., Bertke, M., Lee, J., McCracken, J., O'Toole, T.E., Bhatnagar, A. and Conklin, D.J. (2011). Acrolein Inhalation Prevents Vascular Endothelial Growth Factor-Induced Mobilization of Flk1+/Sca-1+ Cells in Mice. Arterioscl Throm Vas 31: 1598-1606.

75. Smoking \& Cardiovascular Disease (Heart Disease). 2014. Available from: www.heart.org. Accessed November 1, 2017.

76. Buttar, H.S., Li, T. and Ravi, N. (2005). Prevention of cardiovascular diseases: Role of exercise, dietary interventions, obesity and smoking cessation. Experimental \& Clinical Cardiology 10: 229-249.

77. Pope, C.A., 3rd, Burnett, R.T., Krewski, D., Jerrett, M., Shi, Y., Calle, E.E. and Thun, M.J. (2009). Cardiovascular mortality and exposure to airborne fine particulate matter and cigarette smoke: shape of the exposure-response relationship. Circulation 120: 941-948. 
78. Cheah, N.P., Pennings, J.L.A., Vermeulen, J.P., van Schooten, F.J. and Opperhuizen, A. (2013). In vitro effects of aldehydes present in tobacco smoke on gene expression in human lung alveolar epithelial cells. Toxicology in Vitro 27: 1072-1081.

79. Pope, C.A., 3rd, Burnett, R.T., Turner, M.C., Cohen, A., Krewski, D., Jerrett, M., Gapstur, S.M. and Thun, M.J. (2011). Lung cancer and cardiovascular disease mortality associated with ambient air pollution and cigarette smoke: shape of the exposureresponse relationships. Environ Health Perspect 119: 1616-1621.

80. U.S. Department of Health and Human Services. (2014). The Health Consequences of Smoking - 50 Years of Progress: A Report of the Surgeon General, 2014, (Services, U.S.D.o.H.a.H., ed), Office of the Surgeon General, Rockville, MD.

81. U.S. Department of Health and Human Services. (2010). How Tobacco Smoke Causes Disease: The Biology and Behavioral Basis for Smoking-Attributable Disease: A Report of the Surgeon General, (Prevention, C.f.D.C.a., ed), U.S. Centers for Disease Control and Prevention Atlanta, GA.

82. Harmful and Potentially Harmful Constituents in Tobacco Products and Tobacco Smoke: Established List. 2012. Available from: www.fda.gov. Accessed November 6, 2017.

83. Creamer, M.R., Wang, T.W., Babb, S., Cullen, K.A., Day, H., Willis, G., Jamal, A. and Neff, L. (2019). Tobacco Product Use and Cessation Indicators Among AdultsUnited States, 2018. MMWR Morb Mortal Wkly Rep 68: 1013-1019.

84. Health Effects of Secondhand Smoke. 2017. Available from: www.cdc.gov. Accessed November 6, 2017.

85. Ambrose, J.A. and Barua, R.S. (2004). The pathophysiology of cigarette smoking and cardiovascular disease: an update. Journal of the American College of Cardiology 43: 1731-1737.

86. Csordas, A. and Bernhard, D. (2013). The biology behind the atherothrombotic effects of cigarette smoke. Nature reviews. Cardiology 10: 219-230.

87. Barnoya, J. and Glantz, S.A. (2005). Cardiovascular Effects of Secondhand Smoke: Nearly as Large as Smoking. Circulation 111: 2684-2698.

88. Gentner, N.J. and Weber, L.P. (2012). Secondhand tobacco smoke, arterial stiffness, and altered circadian blood pressure patterns are associated with lung inflammation and oxidative stress in rats. American journal of physiology. Heart and circulatory physiology 302: H818-825.

89. Aldehyde. 2018. Available from: www.birtannica.com. Accessed January 22020. 
90. Conklin, D.J., Haberzettl, P., Lee, J. and Srivastava, S. (2011). Chapter 13 Environmental Aldehydes and Cardiovascular Disease, in Environmental Cardiology: Pollution and Heart Disease, pp301-370, The Royal Society of Chemistry.

91. Medical Management Guidelines for Crotonaldehyde. Available from: www.atsdr.cdc.gov. Accessed January 302020.

92. World Health Organization, I.P.o.C.S. (1994). Acetaldehyde: Health and Safety Guide, (Organization, W.H., ed), World Health Organization, Geneva, Switzerland.

93. Kaden, D.A., Mandin, C., Nielsen, G.D., Wolkoff, P. (2010). Formaldehyde, in WHO Guidelines for Indoor Air Quality: Selected Pollutants, World Health Organization, Geneva, Switzerland.

94. Toxicological Profile for Acrolein. 2007. Available from: www.atsdr.cdc.gov. Accessed November 8, 2018.

95. Baker, R.R. (2006). The generation of formaldehyde in cigarettes--Overview and recent experiments. Food and chemical toxicology : an international journal published for the British Industrial Biological Research Association 44: 1799-1822.

96. Ogunwale, M.A., Li, M., Ramakrishnam Raju, M.V., Chen, Y., Nantz, M.H., Conklin, D.J. and Fu, X.-A. (2017). Aldehyde Detection in Electronic Cigarette Aerosols. ACS Omega 2: 1207-1214.

97. Mitkus, R.J., Hess, M.A. and Schwartz, S.L. (2013). Pharmacokinetic modeling as an approach to assessing the safety of residual formaldehyde in infant vaccines. Vaccine 31: 2738-2743.

98. Yu, P.H. and Zuo, D.M. (1996). Formaldehyde produced endogenously via deamination of methylamine. A potential risk factor for initiation of endothelial injury. Atherosclerosis 120: 189-197.

99. World Health Organization. (2000). Formaldehyde, in Air Quality Guidelines for Europe, WHO Regional Publications.

100. U.S. Department of Health and Human Services. (1999). Toxicological Profile for Formaldehyde, (Registry, A.f.T.S.D., ed).

101. Reinhardt, T.E. (1991). Monitoring firefighter exposure to air toxins at prescribed burns of forest and range biomass., (U.S. Department of Agriculture, F.S., ed), Portland, OR.

102. Public Health Statement for Formaldehyde. 2008. Available from: www.atsdr.cdc.gov. Accessed November 6, 2017.

103. World Health Organization. (2002). Formaldehyde, World Health Organization, Geneva, Switzerland. 
104. Formaldehyde and Cancer Risk. 2011. Available from: www.cancer.gov. Accessed November 6, 2017.

105. National Toxicology Program. (2016). Report on Carcinogens, Fourteenth Edition, (Department of Health and Human Services, P.H.S., ed), Research Triangle Park, NC.

106. United States Environmental Protection Agency. (2000). Acetaldehyde Hazard Summary, (Assessment, O.o.H.a.E., ed), Office of Research and Development, Research Triangle Park, NC.

107. Bruinen de Bruin Y, K.D., Kephalopoulos S. (2005). Human exposure characterization of chemical substances (HEXPOC); quantification of exposure routes. , (Centre, E.C.J.R., ed), Office for Official Publication of the European Communities, Italy.

108. Eckert, M., Fleischmann, G., Jira, R., Bolt, H.M. and Golka, K. (2000). Acetaldehyde, in Ullmann's Encyclopedia of Industrial Chemistry, Wiley-VCH Verlag $\mathrm{GmbH} \& \mathrm{Co} . \mathrm{KGaA}$.

109. Talhout, R., Opperhuizen, A. and van Amsterdam, J.G.C. (2007). Role of acetaldehyde in tobacco smoke addiction. European Neuropsychopharmacology 17: 627636.

110. Lynch, C., Lim, C.K., Thomas, M. and Peters, T.J. (1983). Assay of blood and tissue aldehydes by HPLC analysis of their 2,4-dinitrophenylhydrazine adducts. Clinica chimica acta; international journal of clinical chemistry 130: 117-122.

111. Godish, T. (1989). Formaldehyde exposures from tobacco smoke: a review. American Journal of Public Health 79: 1044-1045.

112. van Andel, I., Schenk, E., Rambali, B., Wolterink, G., van de Werken, G., Stevenson, H., van Aerts, L.A.G.J.M. and Vleeming, W. (2017). The health- and addictive effectes due to exposure to aldehydes of cigarette smoke. Part 1; Acetaldehyde, Formaldehyde, Acrolein and Propionaldehyde, (Ministry of Health, W., and Sports, ed), Netherlands National Institute for Public Health and the Environment, Bilthoven, Netherlands.

113. van der Toorn, M., Slebos, D.-J., de Bruin, H.G., Gras, R., Rezayat, D., Jorge, L., Sandra, K. and van Oosterhout, A.J. (2013). Critical role of aldehydes in cigarette smokeinduced acute airway inflammation. Respiratory research 14: 45.

114. Dong, J.Z. and Moldoveanu, S.C. (2004). Gas chromatography-mass spectrometry of carbonyl compounds in cigarette mainstream smoke after derivatization with 2,4-dinitrophenylhydrazine. Journal of chromatography. A 1027: 25-35. 
115. Houlgate, P.R., Dhingra, K.S., Nash, S.J. and Evans, W.H. (1989). Determination of formaldehyde and acetaldehyde in mainstream cigarette smoke by high-performance liquid chromatography. The Analyst 114: 355-360.

116. Borgerding, M.F., Bodnar, J.A., Chung, H.L., Mangan, P.P., Morrison, C.C., Risner, C.H., Rogers, J.C., Simmons, D.F., Uhrig, M.S., Wendelboe, F.N., Wingate, D.E. and Winkler, L.S. (1998). Chemical and biological studies of a new cigarette that primarily heats tobacco. Part 1. Chemical composition of mainstream smoke. Food and chemical toxicology : an international journal published for the British Industrial Biological Research Association 36: 169-182.

117. Jaccard, G., Djoko, D.T., Korneliou, A., Stabbert, R., Belushkin, M. and Esposito, M. (2019). Mainstream smoke constituents and in vitro toxicity comparative analysis of 3R4F and 1R6F reference cigarettes. Toxicology Reports 6: 222-231.

118. Seeman, J.I., Dixon, M. and Haussmann, H.J. (2002). Acetaldehyde in mainstream tobacco smoke: formation and occurrence in smoke and bioavailability in the smoker. Chemical research in toxicology 15: 1331-1350.

119. Centers for Disease Control and Prevention. (2018). Smoking is down, but almost 38 million American adults still smoke, U.S. Department of Health and Human Services.

120. Bassig, B.A., Zhang, L., Vermeulen, R., Tang, X., Li, G., Hu, W., Guo, W., Purdue, M.P., Yin, S., Rappaport, S.M., Shen, M., Ji, Z., Qiu, C., Ge, Y., Hosgood, H., Reiss, B., Wu, B., Xie, Y., Li, L., Yue, F., Freeman, L.E., Blair, A., Hayes, R.B., Huang, H., Smith, M.T., Rothman, N. and Lan, Q. (2016). Comparison of hematological alterations and markers of B-cell activation in workers exposed to benzene, formaldehyde and trichloroethylene. Carcinogenesis 37: 692-700.

121. Kuo, H., Jian, G., Chen, C., Liu, C. and Lai, J. (1997). White blood cell count as an indicator of formaldehyde exposure. Bulletin of environmental contamination and toxicology 59: 261-267.

122. Zhang, L., Tang, X., Rothman, N., Vermeulen, R., Ji, Z., Shen, M., Qiu, C., Guo, W., Liu, S., Reiss, B., Freeman, L.B., Ge, Y., Hubbard, A.E., Hua, M., Blair, A., Galvan, N., Ruan, X., Alter, B.P., Xin, K.X., Li, S., Moore, L.E., Kim, S., Xie, Y., Hayes, R.B., Azuma, M., Hauptmann, M., Xiong, J., Stewart, P., Li, L., Rappaport, S.M., Huang, H., Fraumeni, J.F., Jr., Smith, M.T. and Lan, Q. (2010). Occupational exposure to formaldehyde, hematotoxicity, and leukemia-specific chromosome changes in cultured myeloid progenitor cells. Cancer epidemiology, biomarkers \& prevention : a publication of the American Association for Cancer Research, cosponsored by the American Society of Preventive Oncology 19: 80-88.

123. Strubelt, O., Brasch, H., Pentz, R. and Younes, M. (1990). Experimental studies on the acute cardiovascular toxicity of formalin and its antidotal treatment. Journal of toxicology. Clinical toxicology 28: 221-233. 
124. Tani, T. (1981). Relaxation of vascular smooth muscle induced by formaldehyde (author's transl). Nihon Yakurigaku Zasshi 77: 221-230.

125. Zhang, Q., Tian, P., Zhai, M., Lei, X., Yang, Z., Liu, Y., Liu, M., Huang, H., Zhang, X., Yang, X., Zhao, Y. and Meng, Z. (2018). Formaldehyde regulates vascular tensions through nitric oxide-cGMP signaling pathway and ion channels. Chemosphere 193: 60-73.

126. Conklin, D.J., Cowley, H.R., Wiechmann, R.J., Johnson, G.H., Trent, M.B. and Boor, P.J. (2004). Vasoactive effects of methylamine in isolated human blood vessels: role of semicarbazide-sensitive amine oxidase, formaldehyde, and hydrogen peroxide. American journal of physiology. Heart and circulatory physiology 286: H667-H676.

127. Jin, L., Jagatheesan, G., Guo, L., Nystoriak, M., Malovichko, M., Lorkiewicz, P., Bhatnagar, A., Srivastava, S. and Conklin, D.J. (2019). Formaldehyde Induces Mesenteric Artery Relaxation via a Sensitive Transient Receptor Potential Ankyrin-1 (TRPA1) and Endothelium-Dependent Mechanism: Potential Role in Postprandial Hyperemia. Frontiers in Physiology 10: 277.

128. Institute, R.T., Substances, U.S.A.f.T. and Registry, D. (1999). Toxicological Profile for Formaldehyde. U.S. Department of Health and Human Services, Public Health Service, Agency for Toxic Substances and Disease Registry.

129. Levine, R.J., Andjelkovich, D.A. and Shaw, L.K. (1984). The mortality of Ontario undertakers and a review of formaldehyde-related mortality studies. Journal of occupational medicine. : official publication of the Industrial Medical Association 26: $740-746$.

130. Walrath, J. and Fraumeni, J.F., Jr. (1983). Mortality patterns among embalmers. International journal of cancer 31: 407-411.

131. Guberan, E. and Raymond, L. (1985). Mortality and cancer incidence in the perfumery and flavour industry of Geneva. British journal of industrial medicine 42: 240245.

132. Shin, M.J., Cho, Y. and Davey Smith, G. (2017). Alcohol Consumption, Aldehyde Dehydrogenase 2 Gene Polymorphisms, and Cardiovascular Health in Korea. Yonsei medical journal 58: 689-696.

133. Gross, E.R., Zambelli, V.O., Small, B.A., Ferreira, J.C.B., Chen, C.-H. and Mochly-Rosen, D. (2015). A personalized medicine approach for Asian Americans with the aldehyde dehydrogenase $2 * 2$ variant. Annual review of pharmacology and toxicology 55: $107-127$.

134. Jia, K., Wang, H. and Dong, P. (2015). Aldehyde dehydrogenase 2 (ALDH2) Glu504Lys polymorphism is associated with hypertension risk in Asians: a metaanalysis. International journal of clinical and experimental medicine 8: 10767-10772. 
135. Zhang, Y. and Ren, J. (2011). ALDH2 in Alcoholic Heart Diseases: Molecular Mechanism and Clinical Implications. Pharmacology \& therapeutics 132: 86-95.

136. Gu, J.Y. and Li, L.W. (2014). ALDH2 Glu504Lys polymorphism and susceptibility to coronary artery disease and myocardial infarction in East Asians: a metaanalysis. Archives of medical research 45: 76-83.

137. Wang, Q., Zhou, S., Wang, L., Lei, M., Wang, Y., Miao, C. and Jin, Y. (2013). ALDH2 rs671 Polymorphism and coronary heart disease risk among Asian populations: a meta-analysis and meta-regression. DNA and cell biology 32: 393-399.

138. Aberle, I.N. and Ren, J. (2003). Experimental Assessment of the Role of Acetaldehyde in Alcoholic Cardiomyopathy. Biological procedures online 5: 1-12.

139. Patnaik, P. (2007). Aldehydes, in A Comprehensive Guide to the Hazardous Properties of Chemical Substances, Third Edition, pp160-192, John Wiley \& Sons, Inc., Hoboken, New Jersey.

140. Niemela, O., Israel, Y., Mizoi, Y., Fukunaga, T. and Eriksson, C.J. (1990). Hemoglobin-acetaldehyde adducts in human volunteers following acute ethanol ingestion. Alcoholism, clinical and experimental research 14: 838-841.

141. Peterson, C.M., Jovanovic-Peterson, L. and Schmid-Formby, F. (1988). Rapid association of acetaldehyde with hemoglobin in human volunteers after low dose ethanol. Alcohol (Fayetteville, N.Y.) 5: 371-374.

142. Stevens, V.J., Fantl, W.J., Newman, C.B., Sims, R.V., Cerami, A. and Peterson, C.M. (1981). Acetaldehyde adducts with hemoglobin. Journal of Clinical Investigation 67: 361-369.

143. Lee, S.E. and Park, Y.S. (2013). Role of lipid peroxidation-derived alpha, betaunsaturated aldehydes in vascular dysfunction. Oxidative medicine and cellular longevity 2013: 629028.

144. Luo, J., Hill, B.G., Gu, Y., Cai, J., Srivastava, S., Bhatnagar, A. and Prabhu, S.D. (2007). Mechanisms of acrolein-induced myocardial dysfunction: implications for environmental and endogenous aldehyde exposure. American journal of physiology. Heart and circulatory physiology 293: H3673-3684.

145. Haussmann, H.J. (2012). Use of hazard indices for a theoretical evaluation of cigarette smoke composition. Chemical research in toxicology 25: 794-810.

146. DeWoskin, R.S., Greenberg, M., Pepelko, W. and Strickland, J. (2003). IRIS Toxicological Review of Acrolein, Washington, D.C.

147. Alwis, K.U., deCastro, B.R., Morrow, J.C. and Blount, B.C. (2015). Acrolein Exposure in U.S. Tobacco Smokers and Non-Tobacco Users: NHANES 2005-2006. Environ Health Perspect 123: 1302-1308. 
148. Smith, D., Cheng, P. and Španěl, P. (2002). Analysis of petrol and diesel vapour and vehicle engine exhaust gases using selected ion flow tube mass spectrometry. Rapid Communications in Mass Spectrometry 16: 1124-1134.

149. Stabbert, R., Dempsey, R., Diekmann, J., Euchenhofer, C., Hagemeister, T., Haussmann, H.-J., Knorr, A., Mueller, B.P., Pospisil, P., Reininghaus, W., Roemer, E., Tewes, F.J. and Veltel, D.J. (2017). Studies on the contributions of smoke constituents, individually and in mixtures, in a range of in vitro bioactivity assays. Toxicology in Vitro 42: 222-246.

150. Conklin, D.J., Haberzettl, P., Prough, R.A. and Bhatnagar, A. (2009).

Glutathione-S-transferase $\mathrm{P}$ protects against endothelial dysfunction induced by exposure to tobacco smoke. American journal of physiology. Heart and circulatory physiology 296: H1586-1597.

151. Conklin, D.J., Malovichko, M.V., Zeller, I., Das, T.P., Krivokhizhina, T.V., Lynch, B.H., Lorkiewicz, P., Agarwal, A., Wickramasinghe, N., Haberzettl, P., Sithu, S.D., Shah, J., O’Toole, T.E., Rai, S.N., Bhatnagar, A. and Srivastava, S. (2017). Biomarkers of Chronic Acrolein Inhalation Exposure in Mice: Implications for Tobacco Product-Induced Toxicity. Toxicological Sciences 158: 263-274.

152. Conklin, D.J., Barski, O.A., Lesgards, J.-F., Juvan, P., Rezen, T., Rozman, D., Prough, R.A., Vladykovskaya, E., Liu, S., Srivastava, S. and Bhatnagar, A. (2010). Acrolein consumption induces systemic dyslipidemia and lipoprotein modification. Toxicology and applied pharmacology 243: 1-12.

153. Srivastava, S., Sithu, S.D., Vladykovskaya, E., Haberzettl, P., Hoetker, D.J., Siddiqui, M.A., Conklin, D.J., D'Souza, S.E. and Bhatnagar, A. (2011). Oral exposure to acrolein exacerbates atherosclerosis in apoE-null mice. Atherosclerosis 215: 301-308.

154. Thompson, L.C., Ledbetter, A.D., Haykal-Coates, N., Cascio, W.E., Hazari, M.S. and Farraj, A.K. (2017). Acrolein Inhalation Alters Myocardial Synchrony and Performance at and Below Exposure Concentrations that Cause Ventilatory Responses. Cardiovascular toxicology 17: 97-108.

155. Grant, R.L.J., A. (2016). Crotonaldehyde (Cis and Trans), (Quality, T.C.o.E., ed).

156. van Andel, I., Sleijffers, A., Schenk, E., Rambali, B., Wolterink, G., van de Werken, G., van Aerts, L.A.G.J.M., Vleeming, W. and van Amsterdam, J.G.C. (2006). Adverse Health Effects of Cigarette Smoke: Aldehydes; Crotonaldehyde, Butyraldehde, Hexanala, and Malonaldehyde, (Laboratory for Toxicology, P.a.G., ed).

157. ToxFAQs for Crotonaldehyde. 2002. Available from: www.atsdr.cdc.gov. Accessed March 26, 2018.

158. International Agency for Research on Cancer. (1995). Crotonaldehyde. 
159. Bagchi, P., Geldner, N., deCastro, B.R., De Jesús, V.R., Park, S.K. and Blount, B.C. (2018). Crotonaldehyde exposure in U.S. tobacco smokers and nonsmokers: NHANES 2005-2006 and 2011-2012. Environmental research 163: 1-9.

160. Pazo, D.Y., Moliere, F., Sampson, M.M., Reese, C.M., Agnew-Heard, K.A., Walters, M.J., Holman, M.R., Blount, B.C., Watson, C. and Chambers, D.M. (2016). Mainstream Smoke Levels of Volatile Organic Compounds in 50 US Domestic Cigarette Brands Smoked with the ISO and Canadian Intense Protocols. Nicotine \& tobacco research : official journal of the Society for Research on Nicotine and Tobacco 18: 18861894.

161. Center for Tobacco Reference Products. Accessed November 82018.

162. U.S. Environmental Protection Agency. (1991). Crotonaldehyde: Weight of Evidence for Cancer.

163. Marescotti, D., Gonzalez Suarez, I., Acali, S., Johne, S., Laurent, A., Frentzel, S., Hoeng, J. and Peitsch, M.C. (2016). High Content Screening Analysis to Evaluate the Toxicological Effects of Harmful and Potentially Harmful Constituents (HPHC). Journal of visualized experiments : JoVE.

164. Pei, Z., Zhuang, Z., Sang, H., Wu, Z., Meng, R., He, E.Y., Scott, G.I., Maris, J.R., Li, R. and Ren, J. (2014). alpha,beta-Unsaturated aldehyde crotonaldehyde triggers cardiomyocyte contractile dysfunction: role of TRPV1 and mitochondrial function. Pharmacological research 82: 40-50.

165. Divine, B.J. (1990). An update on mortality among workers at a 1,3-butadiene facility--preliminary results. Environ Health Perspect 86: 119-128.

166. Matanoski, G.M., Santos-Burgoa, C. and Schwartz, L. (1990). Mortality of a cohort of workers in the styrene-butadiene polymer manufacturing industry (1943-1982). Environ Health Perspect 86: 107-117.

167. Penn, A. and Snyder, C.A. (1996). Butadiene inhalation accelerates arteriosclerotic plaque development in cockerels. Toxicology 113: 351-354.

168. Conklin, D.J., Ogunwale, M.A., Chen, Y., Theis, W.S., Nantz, M.H., Fu, X.-A., Chen, L.-C., Riggs, D.W., Lorkiewicz, P., Bhatnagar, A. and Srivastava, S. (2018). Electronic cigarette-generated aldehydes: The contribution of e-liquid components to their formation and the use of urinary aldehyde metabolites as biomarkers of exposure. Aerosol Science and Technology 52: 1219-1232.

169. Clapp, P.W. and Jaspers, I. (2017). Electronic Cigarettes: Their Constituents and Potential Links to Asthma. Curr Allergy Asthma Rep 17: 79-79.

170. Breland, A.B., Spindle, T., Weaver, M. and Eissenberg, T. (2014). Science and electronic cigarettes: current data, future needs. J Addict Med 8: 223-233. 
171. National Academies of Sciences, E. and Medicine. (2018). Public Health Consequences of E-Cigarettes. The National Academies Press, Washington, DC.

172. Electronic Nicotine Delivery Systems and Electronic Non-Nicotine Delivery Systems (ENDS/ENNDS). 2017. Available from: www.who.int. Accessed March 19 2018.

173. Bertholon, J.F., Becquemin, M.H., Annesi-Maesano, I. and Dautzenberg, B. (2013). Electronic Cigarettes: A Short Review. Respiration 86: 433-438.

174. Sussan, T.E., Gajghate, S., Thimmulappa, R.K., Ma, J., Kim, J.-H., Sudini, K., Consolini, N., Cormier, S.A., Lomnicki, S., Hasan, F., Pekosz, A. and Biswal, S. (2015). Exposure to Electronic Cigarettes Impairs Pulmonary Anti-Bacterial and Anti-Viral Defenses in a Mouse Model. PloS one 10: e0116861.

175. Farsalinos, K.E. and Polosa, R. (2014). Safety evaluation and risk assessment of electronic cigarettes as tobacco cigarette substitutes: a systematic review. Therapeutic Advances in Drug Safety 5: 67-86.

176. Farsalinos, K.E. and Gillman, G. (2017). Carbonyl Emissions in E-cigarette Aerosol: A Systematic Review and Methodological Considerations. Frontiers in Physiology 8: 1119.

177. Korzun, T., Lazurko, M., Munhenzva, I., Barsanti, K.C., Huang, Y., Jensen, R.P., Escobedo, J.O., Luo, W., Peyton, D.H. and Strongin, R.M. (2018). E-Cigarette Airflow Rate Modulates Toxicant Profiles and Can Lead to Concerning Levels of Solvent Consumption. ACS Omega 3: 30-36.

178. Gilbert, H.A. (1965). Smokeless non-tobacco cigarette, (Office, U.S.P.a.T., ed), Gilbert, Herbert A., United States of America.

179. DeVito, E.E. and Krishnan-Sarin, S. (2018). E-cigarettes: Impact of E-Liquid Components and Device Characteristics on Nicotine Exposure. Curr Neuropharmacol 16: 438-459.

180. Goniewicz, M.L., Knysak, J., Gawron, M., Kosmider, L., Sobczak, A., Kurek, J., Prokopowicz, A., Jablonska-Czapla, M., Rosik-Dulewska, C., Havel, C., Jacob, P. and Benowitz, N. (2013). Levels of selected carcinogens and toxicants in vapour from electronic cigarettes. Tobacco Control.

181. Patel, D., Davis, K.C., Cox, S., Bradfield, B., King, B.A., Shafer, P., Caraballo, R. and Bunnell, R. (2016). Reasons for current E-cigarette use among U.S. adults. Preventive medicine 93: 14-20.

182. Gentzke, A.S., Creamer, M., Cullen, K.A., Ambrose, B.K., Willis, G., Jamal, A. and King, B.A. (2019). Vital Signs: Tobacco Product Use Among Middle and High School Students - United States, 2011-2018. MMWR Morb Mortal Wkly Rep 68: 157164. 
183. Cullen, K.A., Gentzke, A.S., Sawdey, M.D., Chang, J.T., Anic, G.M., Wang, T.W., Creamer, M.R., Jamal, A., Ambrose, B.K. and King, B.A. (2019). e-Cigarette Use Among Youth in the United States, 2019. JAMA : the journal of the American Medical Association 322: 2095-2103.

184. The National Academies of Sciences, E., and Medicine,. (2018). New Report One of the Most Comprehensive Studies on Health Effects of E-Cigarettes; Finds That Using E-Cigarettes May Lead Youth to Start Smoking, Adults to Stop Smoking.

185. Cho, J.H. (2017). The association between electronic-cigarette use and selfreported oral symptoms including cracked or broken teeth and tongue and/or inside-cheek pain among adolescents: A cross-sectional study. PloS one 12: e0180506.

186. Palamidas, A., Tsikrika, S., Katsaounou, P.A., Vakali, S., Gennimata, S.-A., Kaltsakas, G., Gratziou, C. and Koulouris, N. (2017). Acute effects of short term use of e-cigarettes on airways physiology and respiratory symptoms in smokers with and without airways obstructive diseases and in healthy non smokers. Tobacco Prevention \& Cessation 3.

187. Gennimata, S.-A., Palamidas, A., Kaltsakas, G., Tsikrika, S., Vakali, S., Gratziou, C. and Koulouris, N. (2012). Acute effect of e-cigarette on pulmonary function in healthy subjects and smokers. European Respiratory Journal 40.

188. Vardavas, C.I., Anagnostopoulos, N., Kougias, M., Evangelopoulou, V., Connolly, G.N. and Behrakis, P.K. (2012). Short-term pulmonary effects of using an electronic cigarette: impact on respiratory flow resistance, impedance, and exhaled nitric oxide. Chest 141: 1400-1406.

189. Shaito, A., Saliba, J., Husari, A., El-Harakeh, M., Chhouri, H., Hashem, Y., Shihadeh, A. and El-Sabban, M. (2017). Electronic Cigarette Smoke Impairs Normal Mesenchymal Stem Cell Differentiation. Scientific Reports 7: 14281.

190. Carnevale, R., Sciarretta, S., Violi, F., Nocella, C., Loffredo, L., Perri, L., Peruzzi, M., Marullo, A.G., De Falco, E., Chimenti, I., Valenti, V., Biondi-Zoccai, G. and Frati, G. (2016). Acute Impact of Tobacco vs Electronic Cigarette Smoking on Oxidative Stress and Vascular Function. Chest 150: 606-612.

191. Moheimani, R.S., Bhetraratana, M., Yin, F., Peters, K.M., Gornbein, J., Araujo, J.A. and Middlekauff, H.R. (2017). Increased Cardiac Sympathetic Activity and Oxidative Stress in Habitual Electronic Cigarette Users: Implications for Cardiovascular Risk. JAMA cardiology 2: 278-284.

192. Lerner, C.A., Sundar, I.K., Watson, R.M., Elder, A., Jones, R., Done, D., Kurtzman, R., Ossip, D.J., Robinson, R., McIntosh, S. and Rahman, I. (2015). Environmental health hazards of e-cigarettes and their components: Oxidants and copper in e-cigarette aerosols. Environmental pollution (Barking, Essex : 1987) 198: 100-107. 
193. Madison, M.C., Landers, C.T., Gu, B.H., Chang, C.Y., Tung, H.Y., You, R., Hong, M.J., Baghaei, N., Song, L.Z., Porter, P., Putluri, N., Salas, R., Gilbert, B.E., Levental, I., Campen, M.J., Corry, D.B. and Kheradmand, F. (2019). Electronic cigarettes disrupt lung lipid homeostasis and innate immunity independent of nicotine. The Journal of clinical investigation 129: 4290-4304.

194. Olfert, I.M., DeVallance, E., Hoskinson, H., Branyan, K.W., Clayton, S., Pitzer, C.R., Sullivan, D.P., Breit, M.J., Wu, Z., Klinkhachorn, P., Mandler, W.K., Erdreich, B.H., Ducatman, B.S., Bryner, R.W., Dasgupta, P. and Chantler, P.D. (2018). Chronic exposure to electronic cigarettes results in impaired cardiovascular function in mice. Journal of applied physiology (Bethesda, Md. : 1985) 124: 573-582.

195. Espinoza-Derout, J., Hasan, K.M., Shao, X.M., Jordan, M.C., Sims, C., Lee, D.L., Sinha, S., Simmons, Z., Mtume, N., Liu, Y., Roos, K.P., Sinha-Hikim, A.P. and Friedman, T.C. (2019). Chronic intermittent electronic cigarette exposure induces cardiac dysfunction and atherosclerosis in apolipoprotein-E knockout mice. American journal of physiology. Heart and circulatory physiology 317: H445-H459.

196. Rao, P., Liu, J. and Springer, M.L. (2020). JUUL and Combusted Cigarettes Comparably Impair Endothelial Function. Tob Regul Sci 6: 30-37.

197. Orzabal, M.R., Lunde-Young, E.R., Ramirez, J.I., Howe, S.Y.F., Naik, V.D., Lee, J., Heaps, C.L., Threadgill, D.W. and Ramadoss, J. (2019). Chronic exposure to e-cig aerosols during early development causes vascular dysfunction and offspring growth deficits. Translational research : the journal of laboratory and clinical medicine 207: 7082.

198. Fernández, E., Ballbè, M., Sureda, X., Fu, M., Saltó, E. and Martínez-Sánchez, J.M. (2015). Particulate Matter from Electronic Cigarettes and Conventional Cigarettes: a Systematic Review and Observational Study. Current Environmental Health Reports 2: 423-429.

199. Lee, M.-S., LeBouf, R.F., Son, Y.-S., Koutrakis, P. and Christiani, D.C. (2017). Nicotine, aerosol particles, carbonyls and volatile organic compounds in tobacco- and menthol-flavored e-cigarettes. Environmental Health 16: 42.

200. Schober, W., Szendrei, K., Matzen, W., Osiander-Fuchs, H., Heitmann, D., Schettgen, T., Jorres, R.A. and Fromme, H. (2014). Use of electronic cigarettes (ecigarettes) impairs indoor air quality and increases FeNO levels of e-cigarette consumers. Int J Hyg Environ Health 217: 628-637.

201. Schripp, T., Markewitz, D., Uhde, E. and Salthammer, T. (2013). Does e-cigarette consumption cause passive vaping? Indoor air 23: 25-31.

202. Soule, E.K., Maloney, S.F., Spindle, T.R., Rudy, A.K., Hiler, M.M. and Cobb, C.O. (2017). Electronic cigarette use and indoor air quality in a natural setting. Tobacco Control 26: 109-112. 
203. Farsalinos, K.E., Voudris, V. and Poulas, K. (2015). Are Metals Emitted from Electronic Cigarettes a Reason for Health Concern? A Risk-Assessment Analysis of Currently Available Literature. International Journal of Environmental Research and Public Health 12: 5215-5232.

204. Hess, C.A., Olmedo, P., Navas-Acien, A., Goessler, W., Cohen, J.E. and Rule, A.M. (2017). E-cigarettes as a source of toxic and potentially carcinogenic metals. Environmental research 152: 221-225.

205. Olmedo, P., Goessler, W., Tanda, S., Grau-Perez, M., Jarmul, S., Aherrera, A., Chen, R., Hilpert, M., Cohen, J.E., Navas-Acien, A. and Rule, A.M. (2018). Metal Concentrations in e-Cigarette Liquid and Aerosol Samples: The Contribution of Metallic Coils. Environ Health Perspect 126: 027010.

206. Canistro, D., Vivarelli, F., Cirillo, S., Babot Marquillas, C., Buschini, A., Lazzaretti, M., Marchi, L., Cardenia, V., Rodriguez-Estrada, M.T., Lodovici, M., Cipriani, C., Lorenzini, A., Croco, E., Marchionni, S., Franchi, P., Lucarini, M., Longo, V., Della Croce, C.M., Vornoli, A., Colacci, A., Vaccari, M., Sapone, A. and Paolini, M. (2017). E-cigarettes induce toxicological effects that can raise the cancer risk. Scientific Reports 7: 2028.

207. Rubinstein, M.L., Delucchi, K., Benowitz, N.L. and Ramo, D.E. (2018). Adolescent Exposure to Toxic Volatile Organic Chemicals From E-Cigarettes. Pediatrics.

208. Kosmider, L., Sobczak, A., Fik, M., Knysak, J., Zaciera, M., Kurek, J. and Goniewicz, M.L. (2014). Carbonyl Compounds in Electronic Cigarette Vapors: Effects of Nicotine Solvent and Battery Output Voltage. Nicotine \& Tobacco Research 16: 13191326.

209. Geiss, O., Bianchi, I. and Barrero-Moreno, J. (2016). Correlation of volatile carbonyl yields emitted by e-cigarettes with the temperature of the heating coil and the perceived sensorial quality of the generated vapours. International Journal of Hygiene and Environmental Health 219: 268-277.

210. Gillman, I.G., Kistler, K.A., Stewart, E.W. and Paolantonio, A.R. (2016). Effect of variable power levels on the yield of total aerosol mass and formation of aldehydes in e-cigarette aerosols. Regul Toxicol Pharmacol 75: 58-65.

211. Goniewicz, M.L., Gawron, M., Smith, D.M., Peng, M., Jacob, P., 3rd and Benowitz, N.L. (2017). Exposure to Nicotine and Selected Toxicants in Cigarette Smokers Who Switched to Electronic Cigarettes: A Longitudinal Within-Subjects Observational Study. Nicotine \& tobacco research : official journal of the Society for Research on Nicotine and Tobacco 19: 160-167.

212. Jensen, R.P., Luo, W., Pankow, J.F., Strongin, R.M. and Peyton, D.H. (2015). Hidden Formaldehyde in E-Cigarette Aerosols. New Engl J Med 372: 392-394. 
213. Klager, S., Vallarino, J., MacNaughton, P., Christiani, D.C., Lu, Q. and Allen, J.G. (2017). Flavoring Chemicals and Aldehydes in E-Cigarette Emissions.

Environmental Science \& Technology 51: 10806-10813.

214. Sleiman, M., Logue, J.M., Montesinos, V.N., Russell, M.L., Litter, M.I., Gundel, L.A. and Destaillats, H. (2016). Emissions from Electronic Cigarettes: Key Parameters Affecting the Release of Harmful Chemicals. Environmental Science \& Technology 50: 9644-9651.

215. Ooi, B.G., Dutta, D., Kazipeta, K. and Chong, N.S. (2019). Influence of the ECigarette Emission Profile by the Ratio of Glycerol to Propylene Glycol in E-Liquid Composition. ACS omega 4: 13338-13348.

216. Wang, P., Chen, W., Liao, J., Matsuo, T., Ito, K., Fowles, J., Shusterman, D., Mendell, M. and Kumagai, K. (2017). A Device-Independent Evaluation of Carbonyl Emissions from Heated Electronic Cigarette Solvents. PloS one 12: e0169811-e0169811.

217. Zhu, S.H., Sun, J.Y., Bonnevie, E., Cummins, S.E., Gamst, A., Yin, L. and Lee, M. (2014). Four hundred and sixty brands of e-cigarettes and counting: implications for product regulation. Tob Control 23 Suppl 3: iii3-9.

218. The Safety Assessment and Regulatory Authority to Use Flavors: Focus on ECigarettes. 2014. Available from: www.femaflavor.org. Accessed October 102019.

219. Kreiss, K. (2014). Work-related spirometric restriction in flavoring manufacturing workers. American journal of industrial medicine 57: 129-137.

220. Barrington-Trimis, J.L., Samet, J.M. and McConnell, R. (2014). Flavorings in Electronic Cigarettes: An Unrecognized Respiratory Health Hazard? JAMA : the journal of the American Medical Association 312: 2493-2494.

221. Macedonia, T.V., Krefft, S.D. and Rose, C.S. (2019). Persistent Severe Fixed Airways Obstruction in a High-Dosing E-cigarette User. J Gen Intern Med: 10.1007/s11606-11019-05462-11600.

222. Chapman, D.G., Casey, D.T., Ather, J.L., Aliyeva, M., Daphtary, N., Lahue, K.G., van der Velden, J.L., Janssen-Heininger, Y.M.W. and Irvin, C.G. (2019). The Effect of Flavored E-cigarettes on Murine Allergic Airways Disease. Scientific reports 9: 13671-13671.

223. Landman, S.T., Dhaliwal, I., Mackenzie, C.A., Martinu, T., Steele, A. and Bosma, K.J. (2019). Life-threatening bronchiolitis related to electronic cigarette use in a Canadian youth. CMAJ 191: E1321-E1331.

224. Blagev, D.P., Harris, D., Dunn, A.C., Guidry, D.W., Grissom, C.K. and Lanspa, M.J. (2019). Clinical presentation, treatment, and short-term outcomes of lung injury associated with e-cigarettes or vaping: a prospective observational cohort study. Lancet (London, England): S0140-6736(0119)32679-32670. 
225. Blount, B.C., Karwowski, M.P., Morel-Espinosa, M., Rees, J., Sosnoff, C., Cowan, E., Gardner, M., Wang, L., Valentin-Blasini, L., Silva, L., De Jesús, V.R., Kuklenyik, Z., Watson, C., Seyler, T., Xia, B., Chambers, D., Briss, P., King, B.A., Delaney, L., Jones, C.M., Baldwin, G.T., Barr, J.R., Thomas, J. and Pirkle, J.L. (2019). Evaluation of Bronchoalveolar Lavage Fluid from Patients in an Outbreak of E-cigarette, or Vaping, Product Use-Associated Lung Injury - 10 States, August-October 2019. MMWR Morb Mortal Wkly Rep 68: 1040-1041.

226. Chatham-Stephens, K., Roguski, K., Jang, Y., Cho, P., Jatlaoui, T.C., Kabbani, S., Glidden, E., Ussery, E.N., Trivers, K.F., Evans, M.E., King, B.A., Rose, D.A., Jones, C.M., Baldwin, G., Delaney, L.J., Briss, P., Ritchey, M.D., Lung Injury Response Epidemiology/Surveillance Task, F. and Lung Injury Response Clinical Task, F. (2019). Characteristics of Hospitalized and Nonhospitalized Patients in a Nationwide Outbreak of E-cigarette, or Vaping, Product Use-Associated Lung Injury - United States, November 2019. MMWR Morb Mortal Wkly Rep 68: 1076-1080.

227. Kalininskiy, A., Bach, C.T., Nacca, N.E., Ginsberg, G., Marraffa, J., Navarette, K.A., McGraw, M.D. and Croft, D.P. (2019). E-cigarette, or vaping, product use associated lung injury (EVALI): case series and diagnostic approach. Lancet Respir Med 7: 1017-1026.

228. Moritz, E.D., Zapata, L.B., Lekiachvili, A., Glidden, E., Annor, F.B., Werner, A.K., Ussery, E.N., Hughes, M.M., Kimball, A., DeSisto, C.L., Kenemer, B., Shamout, M., Garcia, M.C., Reagan-Steiner, S., Petersen, E.E., Koumans, E.H., Ritchey, M.D., King, B.A., Jones, C.M., Briss, P.A., Delaney, L., Patel, A., Polen, K.D., Sives, K., Meaney-Delman, D., Chatham-Stephens, K., Lung Injury Response Epidemiology/Surveillance, G. and Lung Injury Response Epidemiology/Surveillance Task, F. (2019). Update: Characteristics of Patients in a National Outbreak of E-cigarette, or Vaping, Product Use-Associated Lung Injuries - United States, October 2019. MMWR Morb Mortal Wkly Rep 68: 985-989.

229. Navon, L., Jones, C.M., Ghinai, I., King, B.A., Briss, P.A., Hacker, K.A. and Layden, J.E. (2019). Risk Factors for E-Cigarette, or Vaping, Product Use-Associated Lung Injury (EVALI) Among Adults Who Use E-Cigarette, or Vaping, Products Illinois, July-October 2019. MMWR Morb Mortal Wkly Rep 68: 1034-1039.

230. Outbreak of Lung Injury Associated with E-Cigarette Use, or Vaping. 2019. Available from: www.cdc.gov. Accessed October 102019.

231. Pepper, J.K., Ribisl, K.M. and Brewer, N.T. (2016). Adolescents' interest in trying flavoured e-cigarettes. Tobacco Control 25: ii62-ii66.

232. Carpenter, C.M., Wayne, G.F., Pauly, J.L., Koh, H.K. and Connolly, G.N. (2005). New Cigarette Brands With Flavors That Appeal To Youth: Tobacco Marketing Strategies. Health Affairs 24: 1601-1610.

233. Blount, B.C., Karwowski, M.P., Shields, P.G., Morel-Espinosa, M., ValentinBlasini, L., Gardner, M., Braselton, M., Brosius, C.R., Caron, K.T., Chambers, D., 
Corstvet, J., Cowan, E., De Jesús, V.R., Espinosa, P., Fernandez, C., Holder, C., Kuklenyik, Z., Kusovschi, J.D., Newman, C., Reis, G.B., Rees, J., Reese, C., Silva, L., Seyler, T., Song, M.-A., Sosnoff, C., Spitzer, C.R., Tevis, D., Wang, L., Watson, C., Wewers, M.D., Xia, B., Heitkemper, D.T., Ghinai, I., Layden, J., Briss, P., King, B.A., Delaney, L.J., Jones, C.M., Baldwin, G.T., Patel, A., Meaney-Delman, D., Rose, D., Krishnasamy, V., Barr, J.R., Thomas, J. and Pirkle, J.L. (2019). Vitamin E Acetate in Bronchoalveolar-Lavage Fluid Associated with EVALI. New Engl J Med 382: 697-705.

234. Whitmer, G. (2019). Governor Whitmer Takes Bold Action to Protect Michigan Kids from Harmful Effects of Vaping.

235. Baker, C.D. (2019). Governor's Declaration of Emergency.

236. Cuomo, A.M. (2019). Governor Cuomo Announces New York State Implements First-in-the-Nation Ban on Flavored E-Cigarettes.

237. Inslee, J. (2019). EXECUTIVE ORDER 19-03: ADDRESSING THE VAPING USE PUBLIC HEALTH CRISIS.

238. Perry, M. (2019). Governor Bullock Directs Ban on Flavored E-Cigarettes to Address Public Health Emergency.

239. Pair, C. and Kondayen, K. (2019). Governor Brown Issues Temporary Ban on Flavored Vaping Products, Convenes Vaping Public Health Workgroup.

240. Raimondo, G.M. (2019). EXECUTIVE ORDER 19-09: Protecting Rhode Island Youth Against the Harms of Vaping.

241. Lee, W.H., Ong, S.-G., Zhou, Y., Tian, L., Bae, H.R., Baker, N., Whitlatch, A., Mohammadi, L., Guo, H., Nadeau, K.C., Springer, M.L., Schick, S.F., Bhatnagar, A. and Wu, J.C. (2019). Modeling Cardiovascular Risks of E-Cigarettes With Human-Induced Pluripotent Stem Cell-Derived Endothelial Cells. Journal of the American College of Cardiology 73: 2722-2737.

242. Fetterman, J.L., Weisbrod, R.M., Feng, B., Bastin, R., Tuttle, S.T., Holbrook, M., Baker, G., Robertson, R.M., Conklin, D.J., Bhatnagar, A. and Hamburg, N.M. (2018). Flavorings in Tobacco Products Induce Endothelial Cell Dysfunction. Arterioscler Thromb Vasc Biol.

243. Smits, P., Temme, L. and Thien, T. (1993). The cardiovascular interaction between caffeine and nicotine in humans. Clinical pharmacology and therapeutics 54: 194-204.

244. Tanus-Santos, J.E., Toledo, J.C., Cittadino, M., Sabha, M., Rocha, J.C. and Moreno, H., Jr. (2001). Cardiovascular effects of transdermal nicotine in mildly hypertensive smokers. American journal of hypertension 14: 610-614. 
245. Gilbert, D.G., Robinson, J.H., Chamberlin, C.L. and Spielberger, C.D. (1989). Effects of smoking/nicotine on anxiety, heart rate, and lateralization of EEG during a stressful movie. Psychophysiology 26: 311-320.

246. Cellina, G.U., Honour, A.J. and Littler, W.A. (1975). Direct arterial pressure, heart rate, and electrocardiogram during cigarette smoking in unrestricted patients. American heart journal 89: 18-25.

247. Ragueneau, I., Michaud, P., Démolis, J.L., Moryusef, A., Jaillon, P. and FunckBrentano, C. (1999). Effects of cigarette smoking on short-term variability of blood pressure in smoking and non smoking healthy volunteers. Fundam Clin Pharmacol 13: 501-507.

248. Folts, J.D. and Bonebrake, F.C. (1982). The effects of cigarette smoke and nicotine on platelet thrombus formation in stenosed dog coronary arteries: inhibition with phentolamine. Circulation 65: 465-470.

249. Renaud, S., Blache, D., Dumont, E., Thevenon, C. and Wissendanger, T. (1984). Platelet function after cigarette smoking in relation to nicotine and carbon monoxide. Clinical pharmacology and therapeutics 36: 389-395.

250. Wu, X., Zhang, H., Qi, W., Zhang, Y., Li, J., Li, Z., Lin, Y., Bai, X., Liu, X., Chen, X., Yang, H., Xu, C., Zhang, Y. and Yang, B. (2018). Nicotine promotes atherosclerosis via ROS-NLRP3-mediated endothelial cell pyroptosis. Cell Death \& Disease 9: 171.

251. Wang, C., Chen, H., Zhu, W., Xu, Y., Liu, M., Zhu, L., Yang, F., Zhang, L., Liu, X., Zhong, Z., Zhao, J., Jiang, J., Xiang, M., Yu, H., Hu, X., Lu, H. and Wang, J.a. (2017). Nicotine Accelerates Atherosclerosis in Apolipoprotein E-Deficient Mice by Activating $\alpha 7$ Nicotinic Acetylcholine Receptor on Mast Cells. Arterioscl Throm Vas 37: 53-65.

252. El-Hellani, A., Salman, R., El-Hage, R., Talih, S., Malek, N., Baalbaki, R., Karaoghlanian, N., Nakkash, R., Shihadeh, A. and Saliba, N.A. (2018). Nicotine and Carbonyl Emissions From Popular Electronic Cigarette Products: Correlation to Liquid Composition and Design Characteristics. Nicotine \& tobacco research : official journal of the Society for Research on Nicotine and Tobacco 20: 215-223.

253. Raymond, B.H., Collette-Merrill, K., Harrison, R.G., Jarvis, S. and Rasmussen, R.J. (2018). The Nicotine Content of a Sample of E-cigarette Liquid Manufactured in the United States. J Addict Med 12: 127-131.

254. Goniewicz, M.L., Kuma, T., Gawron, M., Knysak, J. and Kosmider, L. (2013). Nicotine levels in electronic cigarettes. Nicotine \& tobacco research : official journal of the Society for Research on Nicotine and Tobacco 15: 158-166. 
255. Hutzler, C., Paschke, M., Kruschinski, S., Henkler, F., Hahn, J. and Luch, A. (2014). Chemical hazards present in liquids and vapors of electronic cigarettes. Arch Toxicol 88: 1295-1308.

256. Goldenson, N.I., Leventhal, A.M., Stone, M.D., McConnell, R.S. and BarringtonTrimis, J.L. (2017). Associations of Electronic Cigarette Nicotine Concentration With Subsequent Cigarette Smoking and Vaping Levels in Adolescents. JAMA Pediatrics 171: 1192-1199.

257. Jankowski, M., Krzystanek, M., Zejda, J.E., Majek, P., Lubanski, J., Lawson, J.A. and Brozek, G. (2019). E-Cigarettes are More Addictive than Traditional Cigarettes-A Study in Highly Educated Young People. International journal of environmental research and public health 16: 2279.

258. Farsalinos, K. and Niaura, R. (2019). E-cigarettes and smoking cessation in the United States according to frequency of e-cigarette use and quitting duration: analysis of the 2016 and 2017 National Health Interview Surveys. Nicotine \& tobacco research : official journal of the Society for Research on Nicotine and Tobacco: ntz025.

259. Selya, A.S., Dierker, L., Rose, J.S., Hedeker, D. and Mermelstein, R.J. (2018). The Role of Nicotine Dependence in E-Cigarettes' Potential for Smoking Reduction. Nicotine \& tobacco research : official journal of the Society for Research on Nicotine and Tobacco 20: 1272-1277.

260. Earley, S. (2012). TRPA1 channels in the vasculature. British journal of pharmacology 167: 13-22.

261. Andre, E., Campi, B., Materazzi, S., Trevisani, M., Amadesi, S., Massi, D., Creminon, C., Vaksman, N., Nassini, R., Civelli, M., Baraldi, P.G., Poole, D.P., Bunnett, N.W., Geppetti, P. and Patacchini, R. (2008). Cigarette smoke-induced neurogenic inflammation is mediated by alpha,beta-unsaturated aldehydes and the TRPA1 receptor in rodents. The Journal of clinical investigation 118: 2574-2582.

262. Conklin, D.J. (2016). Acute cardiopulmonary toxicity of inhaled aldehydes: role of TRPA1. Annals of the New York Academy of Sciences 1374: 59-67.

263. Smani, T., Gómez, L.J., Regodon, S., Woodard, G.E., Siegfried, G., Khatib, A.M. and Rosado, J.A. (2018). TRP Channels in Angiogenesis and Other Endothelial Functions. Frontiers in physiology 9: 1731-1731.

264. Taylor-Clark, T.E. (2016). Role of reactive oxygen species and TRP channels in the cough reflex. Cell calcium 60: 155-162.

265. Sullivan, M.N., Gonzales, A.L., Pires, P.W., Bruhl, A., Leo, M.D., Li, W., Oulidi, A., Boop, F.A., Feng, Y., Jaggar, J.H., Welsh, D.G. and Earley, S. (2015). Localized TRPA1 channel $\mathrm{Ca} 2+$ signals stimulated by reactive oxygen species promote cerebral artery dilation. Science Signaling 8: ra2. 
266. Aubdool, A., Kodji, X., Fernandes, E., Bevan, S. and Brain, S.D. (2013). 190 the participation of reactive oxygen species and trpal in cinnamaldehyde-induced vasodilatation in the peripheral vasculature. Heart 99: A106.

267. Hazari, M.S., Haykal-Coates, N., Winsett, D.W., Krantz, Q.T., King, C., Costa, D.L. and Farraj, A.K. (2011). TRPA1 and sympathetic activation contribute to increased risk of triggered cardiac arrhythmias in hypertensive rats exposed to diesel exhaust. Environ Health Persp 119: 951-957.

268. Brône, B., Peeters, P.J., Marrannes, R., Mercken, M., Nuydens, R., Meert, T. and Gijsen, H.J.M. (2008). Tear gasses CN, CR, and CS are potent activators of the human TRPA1 receptor. Toxicology and applied pharmacology 231: 150-156.

269. Conklin, D.J., Haberzettl, P., Jagatheesan, G., Kong, M. and Hoyle, G.W. (2017). Role of TRPA1 in acute cardiopulmonary toxicity of inhaled acrolein. Toxicology and applied pharmacology 324: 61-72.

270. Pozsgai, G., Bodkin, J.V., Graepel, R., Brain, S.D., Andersson, D.A. and Bevan, S. (2010). Evidence for the pathophysiological relevance of TRPA1 receptors in the cardiovascular system in vivo. Cardiovascular research 87: 760-768.

271. Macpherson, L.J., Dubin, A.E., Evans, M.J., Marr, F., Schultz, P.G., Cravatt, B.F. and Patapoutian, A. (2007). Noxious compounds activate TRPA1 ion channels through covalent modification of cysteines. Nature 445: 541-545.

272. Fernandes, E.S., Fernandes, M.A. and Keeble, J.E. (2012). The functions of TRPA1 and TRPV1: moving away from sensory nerves. British journal of pharmacology 166: 510-521.

273. Andersson, K.-E. (2019). TRP Channels as Lower Urinary Tract Sensory Targets. Med Sci (Basel) 7: 67.

274. Barrick, S., Chopra, B., Caterina, M., Nealen, M., Lee, H. and Birder, L. (2005). Expression and function of the cold channels in urinary bladder urothelium: TRPM8 and TRPA1. The Journal of Pain 6: S10.

275. Earley, S., Gonzales, A.L. and Crnich, R. (2009). Endothelium-dependent cerebral artery dilation mediated by TRPA1 and Ca2+-Activated $\mathrm{K}+$ channels. Circ Res 104: 987-994.

276. Sinharoy, P., Bratz, I.N., Sinha, S., Showalter, L.E., Andrei, S.R. and Damron, D.S. (2017). TRPA1 and TRPV1 contribute to propofol-mediated antagonism of U46619-induced constriction in murine coronary arteries. PloS one 12: e0180106.

277. Conklin, D.J., Guo, Y., Nystoriak, M.A., Jagatheesan, G., Obal, D., Kilfoil, P.J., Hoetker, J.D., Guo, L., Bolli, R. and Bhatnagar, A. (2019). TRPA1 channel contributes to myocardial ischemia-reperfusion injury. American Journal of Physiology-Heart and Circulatory Physiology 316: H889-H899. 
278. Andrei, S.R., Sinharoy, P., Bratz, I.N. and Damron, D.S. (2016). TRPA1 is functionally co-expressed with TRPV1 in cardiac muscle: Co-localization at z-discs, costameres and intercalated discs. Channels (Austin) 10: 395-409.

279. Mukhopadhyay, I., Kulkarni, A. and Khairatkar-Joshi, N. (2016). Blocking TRPA1 in Respiratory Disorders: Does It Hold a Promise? Pharmaceuticals (Basel, Switzerland) 9: 70.

280. Bautista, D.M., Jordt, S.E., Nikai, T., Tsuruda, P.R., Read, A.J., Poblete, J., Yamoah, E.N., Basbaum, A.I. and Julius, D. (2006). TRPA1 mediates the inflammatory actions of environmental irritants and proalgesic agents. Cell 124: 1269-1282.

281. Caceres, A.I., Brackmann, M., Elia, M.D., Bessac, B.F., del Camino, D., D'Amours, M., Witek, J.S., Fanger, C.M., Chong, J.A., Hayward, N.J., Homer, R.J., Cohn, L., Huang, X., Moran, M.M. and Jordt, S.E. (2009). A sensory neuronal ion channel essential for airway inflammation and hyperreactivity in asthma. Proceedings of the National Academy of Sciences of the United States of America 106: 9099-9104.

282. Trevisan, G., Benemei, S., Materazzi, S., De Logu, F., De Siena, G., Fusi, C., Fortes Rossato, M., Coppi, E., Marone, I.M., Ferreira, J., Geppetti, P. and Nassini, R. (2016). TRPA1 mediates trigeminal neuropathic pain in mice downstream of monocytes/macrophages and oxidative stress. Brain : a journal of neurology 139: 13611377.

283. Achanta, S. and Jordt, S.-E. (2017). TRPA1: Acrolein meets its target. Toxicology and applied pharmacology 324: 45-50.

284. Russell, F.A., King, R., Smillie, S.J., Kodji, X. and Brain, S.D. (2014). Calcitonin gene-related peptide: physiology and pathophysiology. Physiological reviews 94: 10991142 .

285. Trevisani, M., Siemens, J., Materazzi, S., Bautista, D.M., Nassini, R., Campi, B., Imamachi, N., Andrè, E., Patacchini, R., Cottrell, G.S., Gatti, R., Basbaum, A.I., Bunnett, N.W., Julius, D. and Geppetti, P. (2007). 4-Hydroxynonenal, an endogenous aldehyde, causes pain and neurogenic inflammation through activation of the irritant receptor TRPA1. Proceedings of the National Academy of Sciences 104: 13519-13524.

286. Shapiro, D., Deering-Rice, C.E., Romero, E.G., Hughen, R.W., Light, A.R., Veranth, J.M. and Reilly, C.A. (2013). Activation of transient receptor potential ankyrin1 (TRPA1) in lung cells by wood smoke particulate material. Chemical research in toxicology 26: 750-758.

287. Taylor-Clark, T.E., Kiros, F., Carr, M.J. and McAlexander, M.A. (2009).

Transient receptor potential ankyrin 1 mediates toluene diisocyanate-evoked respiratory irritation. American journal of respiratory cell and molecular biology 40: 756-762.

288. Kichko, T.I., Kobal, G. and Reeh, P.W. (2015). Cigarette smoke has sensory effects through nicotinic and TRPA1 but not TRPV1 receptors on the isolated mouse 
trachea and larynx. American journal of physiology. Lung cellular and molecular physiology 309: L812-L820.

289. Kurhanewicz, N., Ledbetter, A., Farraj, A. and Hazari, M. (2018). TRPA1 mediates the cardiac effects of acrolein through parasympathetic dominance but also sympathetic modulation in mice. Toxicology and applied pharmacology 347: 104-114.

290. Kurhanewicz, N., McIntosh-Kastrinsky, R., Tong, H., Ledbetter, A., Walsh, L., Farraj, A. and Hazari, M. (2017). TRPA1 mediates changes in heart rate variability and cardiac mechanical function in mice exposed to acrolein. Toxicology and applied pharmacology 324: 51-60.

291. Keith, R.J., Fetterman, J.L., Riggs, D.W., O’Toole, T., Nystoriak, J.L., Holbrook, M., Lorkiewicz, P., Bhatnagar, A., DeFilippis, A.P. and Hamburg, N.M. (2018). Protocol to assess the impact of tobacco-induced volatile organic compounds on cardiovascular risk in a cross- sectional cohort: Cardiovascular Injury due to Tobacco Use study. BMJ Open 8.

292. Duda, D.G., Cohen, K.S., Scadden, D.T. and Jain, R.K. (2007). A protocol for phenotypic detection and enumeration of circulating endothelial cells and circulating progenitor cells in human blood. Nat Protoc 2: 805-810.

293. Lorkiewicz, P., Riggs, D.W., Keith, R.J., Conklin, D.J., Xie, Z., Sutaria, S., Lynch, B., Srivastava, S. and Bhatnagar, A. (2018). Comparison of Urinary Biomarkers of Exposure in Humans Using Electronic Cigarettes, Combustible Cigarettes, and Smokeless Tobacco. Nicotine \& Tobacco Research: nty089-nty089.

294. DeJarnett, N., Yeager, R., Conklin, D.J., Lee, J., O'Toole, T.E., McCracken, J., Abplanalp, W., Srivastava, S., Riggs, D.W., Hamzeh, I., Wagner, S., Chugh, A., DeFilippis, A., Ciszewski, T., Wyatt, B., Becher, C., Higdon, D., Ramos, K.S., Tollerud, D.J., Myers, J.A., Rai, S.N., Shah, J., Zafar, N., Krishnasamy, S.S., Prabhu, S.D. and Bhatnagar, A. (2015). Residential Proximity to Major Roadways Is Associated With Increased Levels of AC133+ Circulating Angiogenic Cells. Arterioscl Throm Vas 35: 2468-2477.

295. Yeager, R., Riggs Daniel, W., DeJarnett, N., Tollerud David, J., Wilson, J., Conklin Daniel, J., O'Toole Timothy, E., McCracken, J., Lorkiewicz, P., Xie, Z., Zafar, N., Krishnasamy Sathya, S., Srivastava, S., Finch, J., Keith Rachel, J., DeFilippis, A., Rai Shesh, N., Liu, G. and Bhatnagar, A. (2018). Association Between Residential Greenness and Cardiovascular Disease Risk. Journal of the American Heart Association 7: e009117.

296. Wickham, H., Averick, M., Bryan, J., Chang, W., McGowan, L.D., Francois, R., Grolemund, G., Hayes, A., Henry, L., Hester, J., Kuhn, M., Pedersen, T.L., Miller, E., Bache, S.M., Muller, K., Ooms, J., Robinson, D., Seidel, D.P., Spinu, V., Takahashi, K., Vaughan, D., Wilke, C., Woo, K. and Yutani, H. (2019). Welcome to the Tidyverse. Journal of Open Source Software 4: 1686-1691. 
297. forcats: Tools for working with categorical variables (factors). 2019. Available from: https://cran.r-project.org/package=forcats2020.

298. Liaw, A. and Wiener, M. (2002). Classification and Regression by randomForest. R News 2/3: 18-22.

299. Haberzettl, P., Conklin, D.J., Abplanalp, W.T., Bhatnagar, A. and O'Toole, T.E. (2018). Inhalation of Fine Particulate Matter Impairs Endothelial Progenitor Cell Function Via Pulmonary Oxidative Stress. Arterioscler Thromb Vasc Biol 38: 131-142.

300. Conklin, D.J., Haberzettl, P., Lesgards, J.-F., Prough, R.A., Srivastava, S. and Bhatnagar, A. (2009). Increased sensitivity of glutathione S-transferase P-null mice to cyclophosphamide-induced urinary bladder toxicity. The Journal of pharmacology and experimental therapeutics 331: 456-469.

301. Kage, S., Kudo, K., Ikeda, H. and Ikeda, N. (2004). Simultaneous determination of formate and acetate in whole blood and urine from humans using gas chromatographymass spectrometry. Journal of chromatography. B, Analytical technologies in the biomedical and life sciences 805: 113-117.

302. Lamarre, S.G., MacMillan, L., Morrow, G.P., Randell, E., Pongnopparat, T., Brosnan, M.E. and Brosnan, J.T. (2014). An isotope-dilution, GC-MS assay for formate and its application to human and animal metabolism. Amino acids 46: 1885-1891.

303. de Jesús, V.R. CDC Laboratory Procedure Manual: Ultra Performance Liquid Chromatography with Electro Spray Tandem Mass Spectrometry [UPLC ESI/MSMS] (Method No. 2103), (Sciences, D.o.L., ed), Centers for Disease Control and Prevention.

304. American Heart Association, A.S.A. (2017). Cardiovascular disease: a costly burden for America (projections through 2035), (Association, A.H., ed), Washington, DC.

305. Giannotti, G., Doerries, C., Mocharla, P.S., Mueller, M.F., Bahlmann, F.H., Horvath, T., Jiang, H., Sorrentino, S.A., Steenken, N., Manes, C., Marzilli, M., Rudolph, K.L., Luscher, T.F., Drexler, H. and Landmesser, U. (2010). Impaired endothelial repair capacity of early endothelial progenitor cells in prehypertension: relation to endothelial dysfunction. Hypertension 55: 1389-1397.

306. Wang, X., Chen, J., Tao, Q., Zhu, J. and Shang, Y. (2004). Effects of ox-LDL on number and activity of circulating endothelial progenitor cells. Drug and chemical toxicology 27: 243-255.

307. Tepper, O.M., Galiano, R.D., Capla, J.M., Kalka, C., Gagne, P.J., Jacobowitz, G.R., Levine, J.P. and Gurtner, G.C. (2002). Human endothelial progenitor cells from type II diabetics exhibit impaired proliferation, adhesion, and incorporation into vascular structures. Circulation 106: 2781-2786. 
308. Fadini, G.P., Miorin, M., Facco, M., Bonamico, S., Baesso, I., Grego, F., Menegolo, M., de Kreutzenberg, S.V., Tiengo, A., Agostini, C. and Avogaro, A. (2005). Circulating endothelial progenitor cells are reduced in peripheral vascular complications of type 2 diabetes mellitus. Journal of the American College of Cardiology 45: 14491457.

309. Powell, T.M., Paul, J.D., Hill, J.M., Thompson, M., Benjamin, M., Rodrigo, M., McCoy, J.P., Read, E.J., Khuu, H.M., Leitman, S.F., Finkel, T. and Cannon, R.O., 3rd. (2005). Granulocyte colony-stimulating factor mobilizes functional endothelial progenitor cells in patients with coronary artery disease. Arterioscler Thromb Vasc Biol 25: 296-301.

310. Pellegatta, F., Bragheri, M., Grigore, L., Raselli, S., Maggi, F.M., Brambilla, C., Reduzzi, A., Pirillo, A., Norata, G.D. and Catapano, A.L. (2006). In vitro isolation of circulating endothelial progenitor cells is related to the high density lipoprotein plasma levels. International journal of molecular medicine 17: 203-208.

311. Kunz, G.A., Liang, G., Cuculi, F., Gregg, D., Vata, K.C., Shaw, L.K., Goldschmidt-Clermont, P.J., Dong, C., Taylor, D.A. and Peterson, E.D. (2006). Circulating endothelial progenitor cells predict coronary artery disease severity. Am Heart J 152: 190-195.

312. Thum, T., Tsikas, D., Stein, S., Schultheiss, M., Eigenthaler, M., Anker, S.D., Poole-Wilson, P.A., Ertl, G. and Bauersachs, J. (2005). Suppression of endothelial progenitor cells in human coronary artery disease by the endogenous nitric oxide synthase inhibitor asymmetric dimethylarginine. Journal of the American College of Cardiology 46: 1693-1701.

313. Guven, H., Shepherd, R.M., Bach, R.G., Capoccia, B.J. and Link, D.C. (2006). The number of endothelial progenitor cell colonies in the blood is increased in patients with angiographically significant coronary artery disease. Journal of the American College of Cardiology 48: 1579-1587.

314. Hristov, M., Fach, C., Becker, C., Heussen, N., Liehn, E.A., Blindt, R., Hanrath, P. and Weber, C. (2007). Reduced numbers of circulating endothelial progenitor cells in patients with coronary artery disease associated with long-term statin treatment. Atherosclerosis 192: 413-420.

315. Mobarrez, F., Antoniewicz, L., Bosson, J.A., Kuhl, J., Pisetsky, D.S. and Lundback, M. (2014). The effects of smoking on levels of endothelial progenitor cells and microparticles in the blood of healthy volunteers. PloS one 9: e90314.

316. Michaud, S.E., Dussault, S., Haddad, P., Groleau, J. and Rivard, A. (2006). Circulating endothelial progenitor cells from healthy smokers exhibit impaired functional activities. Atherosclerosis 187: 423-432.

317. Johnson, H.M., Gossett, L.K., Piper, M.E., Aeschlimann, S.E., Korcarz, C.E., Baker, T.B., Fiore, M.C. and Stein, J.H. (2010). Effects of smoking and smoking 
cessation on endothelial function: 1-year outcomes from a randomized clinical trial. Journal of the American College of Cardiology 55: 1988-1995.

318. Kondo, T., Hayashi, M., Takeshita, K., Numaguchi, Y., Kobayashi, K., Iino, S., Inden, Y. and Murohara, T. (2004). Smoking cessation rapidly increases circulating progenitor cells in peripheral blood in chronic smokers. Arterioscler Thromb Vasc Biol 24: $1442-1447$.

319. Puls, M., Schroeter, M.R., Steier, J., Stijohann, L., Hasenfuss, G., Konstantinides, S. and Schafer, K. (2011). Effect of smoking cessation on the number and adhesive properties of early outgrowth endothelial progenitor cells. International journal of cardiology 152: 61-69.

320. Li, W., Du, D.-Y., Liu, Y., Jiang, F., Zhang, P. and Li, Y.-T. (2017). Long-term nicotine exposure induces dysfunction of mouse endothelial progenitor cells.

Experimental and Therapeutic Medicine 13: 85-90.

321. Malovichko, M.V., Zeller, I., Krivokhizhina, T.V., Xie, Z., Lorkiewicz, P., Agarwal, A., Wickramasinghe, N., Sithu, S.D., Shah, J., O’Toole, T., Rai, S.N., Bhatnagar, A., Conklin, D.J. and Srivastava, S. (2017). Systemic Toxicity of Smokeless Tobacco Products in Mice. Nicotine \& Tobacco Research: ntx230-ntx230.

322. Antoniewicz, L., Bosson, J.A., Kuhl, J., Abdel-Halim, S.M., Kiessling, A., Mobarrez, F. and Lundback, M. (2016). Electronic cigarettes increase endothelial progenitor cells in the blood of healthy volunteers. Atherosclerosis 255: 179-185.

323. Farsalinos, K.E. and Polosa, R. (2017). Endothelial progenitor cell release is usually considered a beneficial effect: Problems in interpreting the acute effects of ecigarette use. Atherosclerosis 258: 162-163.

324. Heiss, C. (2016). Electronic cigarettes increase EPCs. Atherosclerosis 255: 119121.

325. Wang, T.W., Asman, K., Gentzke, A.S., Cullen, K.A., Holder-Hayes, E., ReyesGuzman, C., Jamal, A., Neff, L. and King, B.A. (2018). Tobacco Product Use Among Adults - United States, 2017. MMWR Morb Mortal Wkly Rep 67: 1225-1232.

326. Society, A.C. (2019). American Cancer Society Position Statement on Electronic Cigarettes.

327. Pinto, E. (2007). Blood pressure and ageing. Postgrad Med J 83: 109-114.

328. Uranga, R.M. and Keller, J.N. (2010). Diet and age interactions with regards to cholesterol regulation and brain pathogenesis. Curr Gerontol Geriatr Res 2010: 219683219683.

329. Mikkelsen, K.L., Wiinberg, N., Høegholm, A., Christensen, H.R., Bang, L.E., Nielsen, P.E., Svendsen, T.L., Kampmann, J.P., Madsen, N.H. and Bentzon, M.W. 
(1997). Smoking related to 24-h ambulatory blood pressure and heart rate: a study in 352 normotensive Danish subjects. American journal of hypertension 10: 483-491.

330. Benowitz, N.L. and Sharp, D.S. (1989). Inverse relation between serum cotinine concentration and blood pressure in cigarette smokers. Circulation 80: 1309-1312.

331. Li, G., Wang, H., Wang, K., Wang, W., Dong, F., Qian, Y., Gong, H., Hui, C., Xu, G., Li, Y., Pan, L., Zhang, B. and Shan, G. (2017). The association between smoking and blood pressure in men: a cross-sectional study. BMC Public Health 17: 797-797.

332. Green, M.S., Jucha, E. and Luz, Y. (1986). Blood pressure in smokers and nonsmokers: Epidemiologic findings. American Heart Journal 111: 932-940.

333. Lee, W.E. (1908). THE ACTION OF TOBACCO SMOKE, WITH SPECIAL REFERENCE TO ARTERIAL PRESSURE AND DEGENERATION. Quarterly Journal of Experimental Physiology 1: 335-358.

334. Groppelli, A., Giorgi, D.M., Omboni, S., Parati, G. and Mancia, G. (1992).

Persistent blood pressure increase induced by heavy smoking. Journal of hypertension 10: 495-499.

335. Dochi, M., Sakata, K., Oishi, M., Tanaka, K., Kobayashi, E. and Suwazono, Y. (2009). Smoking as an Independent Risk Factor for Hypertension: A 14-Year Longitudinal Study in Male Japanese Workers. The Tohoku Journal of Experimental Medicine 217: 37-43.

336. Lee, D.-H., Ha, M.-H., Kim, J.-R. and Jacobs David, R. (2001). Effects of Smoking Cessation on Changes in Blood Pressure and Incidence of Hypertension. Hypertension 37: 194-198.

337. Mitchell, G.F. (2014). Arterial stiffness and hypertension: chicken or egg? Hypertension (Dallas, Tex. : 1979) 64: 210-214.

338. Doonan, R.J., Hausvater, A., Scallan, C., Mikhailidis, D.P., Pilote, L. and Daskalopoulou, S.S. (2010). The effect of smoking on arterial stiffness. Hypertension Research 33: 398-410.

339. Mahmud, A. and Feely, J. (2003). Effect of Smoking on Arterial Stiffness and Pulse Pressure Amplification. Hypertension 41: 183-187.

340. Levent, E., Ozyürek, A.R. and Ülger, Z. (2004). Evaluation of aortic stiffness in tobacco-smoking adolescents. Journal of Adolescent Health 34: 339-343.

341. Kallio, K., Jokinen, E., Hämäläinen, M., Saarinen, M., Volanen, I., Kaitosaari, T., Viikari, J., Rönnemaa, T., Simell, O. and Raitakari, O.T. (2009). Decreased Aortic Elasticity in Healthy 11-Year-Old Children Exposed to Tobacco Smoke. Pediatrics 123: e267. 
342. Jatoi Noor, A., Jerrard-Dunne, P., Feely, J. and Mahmud, A. (2007). Impact of Smoking and Smoking Cessation on Arterial Stiffness and Aortic Wave Reflection in Hypertension. Hypertension 49: 981-985.

343. Schwartz, J. and Weiss, S.T. (1994). Cigarette smoking and peripheral blood leukocyte differentials. Annals of epidemiology 4: 236-242.

344. Hoonhorst, S.J.M., Timens, W., Koenderman, L., Lo Tam Loi, A.T., Lammers, J.W.J., Boezen, H.M., van Oosterhout, A.J.M., Postma, D.S. and Ten Hacken, N.H.T. (2014). Increased activation of blood neutrophils after cigarette smoking in young individuals susceptible to COPD. Respiratory research 15: 121-121.

345. Pedersen, K.M., Çolak, Y., Ellervik, C., Hasselbalch, H.C., Bojesen, S.E. and Nordestgaard, B.G. (2019). Smoking and Increased White and Red Blood Cells. Arterioscl Throm Vas 39: 965-977.

346. Agarwal, C., Baron, T. and Mehta, D. (2016). Hidden truth of circulating neutrophils (polymorphonuclear neutrophil) function in periodontally healthy smoker subjects. Journal of Indian Society of Periodontology 20: 157-160.

347. Xu, M., Scott, J.E., Liu, K.-Z., Bishop, H.R., Renaud, D.E., Palmer, R.M., SoussiGounni, A. and Scott, D.A. (2008). The influence of nicotine on granulocytic differentiation - inhibition of the oxidative burst and bacterial killing and increased matrix metalloproteinase-9 release. BMC cell biology 9: 19-19.

348. Rosales, C. (2018). Neutrophil: A Cell with Many Roles in Inflammation or Several Cell Types? Frontiers in physiology 9: 113-113.

349. Mortaz, E., Alipoor, S.D., Adcock, I.M., Mumby, S. and Koenderman, L. (2018). Update on Neutrophil Function in Severe Inflammation. Front Immunol 9: 2171-2171.

350. Wright, H.L., Moots, R.J., Bucknall, R.C. and Edwards, S.W. (2010). Neutrophil function in inflammation and inflammatory diseases. Rheumatology 49: 1618-1631.

351. Van Tiel, E., Peeters, P.H., Smit, H.A., Nagelkerke, N.J., Van Loon, A.J., Grobbee, D.E. and Bueno-de-Mesquita, H.B. (2002). Quitting smoking may restore hematological characteristics within five years. Annals of epidemiology 12: 378-388.

352. Lao, X.Q., Jiang, C.Q., Zhang, W.S., Adab, P., Lam, T.H., Cheng, K.K. and Thomas, G.N. (2009). Smoking, smoking cessation and inflammatory markers in older Chinese men: The Guangzhou Biobank Cohort Study. Atherosclerosis 203: 304-310.

353. Smith, M.R., Kinmonth, A.L., Luben, R.N., Bingham, S., Day, N.E., Wareham, N.J., Welch, A. and Khaw, K.T. (2003). Smoking status and differential white cell count in men and women in the EPIC-Norfolk population. Atherosclerosis 169: 331-337. 
354. Higuchi, T., Omata, F., Tsuchihashi, K., Higashioka, K., Koyamada, R. and Okada, S. (2016). Current cigarette smoking is a reversible cause of elevated white blood cell count: Cross-sectional and longitudinal studies. Prev Med Rep 4: 417-422.

355. Malenica, M., Prnjavorac, B., Bego, T., Dujic, T., Semiz, S., Skrbo, S., Gusic, A., Hadzic, A. and Causevic, A. (2017). Effect of Cigarette Smoking on Haematological Parameters in Healthy Population. Med Arch 71: 132-136.

356. Yanbaeva, D.G., Dentener, M.A., Creutzberg, E.C., Wesseling, G. and Wouters, E.F.M. (2007). Systemic Effects of Smoking. Chest 131: 1557-1566.

357. Fernández, J.A.F., Prats, J.M., Artero, J.V.M., Mora, A.C., Fariñas, A.V., Espinal, A. and Méndez, J.A.G. (2012). Systemic inflammation in 222.841 healthy employed smokers and nonsmokers: white blood cell count and relationship to spirometry. Tobacco Induced Diseases 10: 7.

358. GEORGE, E.L. and PANOS, A. (2005). Does a high WBC count signal infection? Nursing2020 35: 20-21.

359. Flouris, A.D., Poulianiti, K.P., Chorti, M.S., Jamurtas, A.Z., Kouretas, D., Owolabi, E.O., Tzatzarakis, M.N., Tsatsakis, A.M. and Koutedakis, Y. (2012). Acute effects of electronic and tobacco cigarette smoking on complete blood count. Food and Chemical Toxicology 50: 3600-3603.

360. Okuni-Watanabe, M., Kurata, K. and Yakushijin, K. (2019). The First Case of ECigarette-Induced Polycythemia. Case Rep Hematol 2019: 2084325-2084325.

361. Kurtoğlu, E., Aktürk, E., Korkmaz, H., Sincer, I., Yılmaz, M., Erdem, K., Celik, A. and Ozdemir, R. (2013). Elevated red blood cell distribution width in healthy smokers. Turk Kardiyol Dern Ars 41: 199-206.

362. Patel, K.V., Ferrucci, L., Ershler, W.B., Longo, D.L. and Guralnik, J.M. (2009). Red Blood Cell Distribution Width and the Risk of Death in Middle-aged and Older Adults. Archives of Internal Medicine 169: 515-523.

363. Ozgul, G., Seyhan, E.C., Ozgul, M.A. and Gunluoglu, M.Z. (2017). Red Blood Cell Distribution Width in Patients With Chronic Obstructive Pulmonary Disease and Healthy Subjects. Archivos de Bronconeumología (English Edition) 53: 107-113.

364. Felker, G.M., Allen, L.A., Pocock, S.J., Shaw, L.K., McMurray, J.J.V., Pfeffer, M.A., Swedberg, K., Wang, D., Yusuf, S., Michelson, E.L. and Granger, C.B. (2007). Red Cell Distribution Width as a Novel Prognostic Marker in Heart Failure. Journal of the American College of Cardiology 50: 40.

365. Tonelli, M., Sacks, F., Arnold, M., Moye, L., Davis, B. and Pfeffer, M. (2008). Relation Between Red Blood Cell Distribution Width and Cardiovascular Event Rate in People With Coronary Disease. Circulation 117: 163-168. 
366. Perlstein, T.S., Weuve, J., Pfeffer, M.A. and Beckman, J.A. (2009). Red Blood Cell Distribution Width and Mortality Risk in a Community-Based Prospective Cohort. Archives of Internal Medicine 169: 588-594.

367. Skjelbakken, T., Lappegård, J., Ellingsen, T.S., Barrett-Connor, E., Brox, J., Løchen, M.-L., Njølstad, I., Wilsgaard, T., Mathiesen, E.B., Brækkan, S.K. and Hansen, J.-B. (2014). Red cell distribution width is associated with incident myocardial infarction in a general population: the Troms $\varnothing$ Study. Journal of the American Heart Association 3: e001109.

368. Glynn, M.F., Mustard, J.F., Buchanan, M.R. and Murphy, E.A. (1966). Cigarette smoking and platelet aggregation. Can Med Assoc J 95: 549-553.

369. Bridges, A.B., Hill, A. and Belch, J.J. (1993). Cigarette smoking increases white blood cell aggregation in whole blood. J R Soc Med 86: 139-140.

370. Hom, S., Chen, L., Wang, T., Ghebrehiwet, B., Yin, W. and Rubenstein, D.A. (2016). Platelet activation, adhesion, inflammation, and aggregation potential are altered in the presence of electronic cigarette extracts of variable nicotine concentrations. Platelets 27: 694-702.

371. Qasim, H., Karim, Z.A., Silva-Espinoza, J.C., Khasawneh, F.T., Rivera, J.O., Ellis, C.C., Bauer, S.L., Almeida, I.C. and Alshbool, F.Z. (2018). Short-Term E-Cigarette Exposure Increases the Risk of Thrombogenesis and Enhances Platelet Function in Mice. Journal of the American Heart Association 7: e009264.

372. Nocella, C., Biondi-Zoccai, G., Sciarretta, S., Peruzzi, M., Pagano, F., Loffredo, L., Pignatelli, P., Bullen, C., Frati, G. and Carnevale, R. (2018). Impact of Tobacco Versus Electronic Cigarette Smoking on Platelet Function. The American journal of cardiology 122: 1477-1481.

373. Fagerström, K. (2005). The Nicotine Market: An Attempt to Estimate the Nicotine Intake from Various Sources and the Total Nicotine Consumption in Some Countries. Nicotine \& Tobacco Research 7: 343-350.

374. Grana, R., Benowitz, N. and Glantz, S.A. (2014). E-cigarettes: a scientific review. Circulation 129: 1972-1986.

375. St Helen, G., Liakoni, E., Nardone, N., Addo, N., Jacob, P., 3rd and Benowitz, N.L. (2020). Comparison of Systemic Exposure to Toxic and/or Carcinogenic Volatile Organic Compounds (VOC) during Vaping, Smoking, and Abstention. Cancer Prev Res (Phila) 13: 153-162.

376. Zhang, G., Wang, Z., Zhang, K., Hou, R., Xing, C., Yu, Q. and Liu, E. (2018). Safety Assessment of Electronic Cigarettes and Their Relationship with Cardiovascular Disease. International journal of environmental research and public health 15: 75. 
377. Worku, D. and Worku, E. (2019). A narrative review evaluating the safety and efficacy of e-cigarettes as a newly marketed smoking cessation tool. SAGE Open Med 7: $2050312119871405-2050312119871405$.

378. Chaffee, B.W. (2019). Electronic Cigarettes: Trends, Health Effects and Advising Patients Amid Uncertainty. J Calif Dent Assoc 47: 85-92.

379. Wilson, P.W., D'Agostino, R.B., Levy, D., Belanger, A.M., Silbershatz, H. and Kannel, W.B. (1998). Prediction of coronary heart disease using risk factor categories. Circulation 97: 1837-1847.

380. Conroy, R.M., Pyorala, K., Fitzgerald, A.P., Sans, S., Menotti, A., De Backer, G., De Bacquer, D., Ducimetiere, P., Jousilahti, P., Keil, U., Njolstad, I., Oganov, R.G., Thomsen, T., Tunstall-Pedoe, H., Tverdal, A., Wedel, H., Whincup, P., Wilhelmsen, L. and Graham, I.M. (2003). Estimation of ten-year risk of fatal cardiovascular disease in Europe: the SCORE project. European heart journal 24: 987-1003.

381. Assmann, G., Cullen, P. and Schulte, H. (2002). Simple scoring scheme for calculating the risk of acute coronary events based on the 10-year follow-up of the prospective cardiovascular Munster (PROCAM) study. Circulation 105: 310-315.

382. Diamond, G.A. and Forrester, J.S. (1979). Analysis of Probability as an Aid in the Clinical Diagnosis of Coronary-Artery Disease. New Engl J Med 300: 1350-1358.

383. Donato, A.J., Lesniewski, L.A. and Delp, M.D. (2005). The effects of aging and exercise training on endothelin-1 vasoconstrictor responses in rat skeletal muscle arterioles. Cardiovascular research 66: 393-401.

384. Seals, D.R., Jablonski, K.L. and Donato, A.J. (2011). Aging and vascular endothelial function in humans. Clinical science (London, England : 1979) 120: 357-375.

385. Williamson, K., Stringer, S. and Alexander, Y. (2012). Endothelial Progenitor Cells Enter the Aging Arena. Frontiers in Physiology 3.

386. Heiss, C., Keymel, S., Niesler, U., Ziemann, J., Kelm, M. and Kalka, C. (2005). Impaired progenitor cell activity in age-related endothelial dysfunction. Journal of the American College of Cardiology 45: 1441-1448.

387. Schultz William, M., Kelli Heval, M., Lisko John, C., Varghese, T., Shen, J., Sandesara, P., Quyyumi Arshed, A., Taylor Herman, A., Gulati, M., Harold John, G., Mieres Jennifer, H., Ferdinand Keith, C., Mensah George, A. and Sperling Laurence, S. (2018). Socioeconomic Status and Cardiovascular Outcomes. Circulation 137: 21662178.

388. Psaltopoulou, T., Hatzis, G., Papageorgiou, N., Androulakis, E., Briasoulis, A. and Tousoulis, D. (2017). Socioeconomic status and risk factors for cardiovascular disease: Impact of dietary mediators. Hellenic journal of cardiology : HJC $=$ Hellenike kardiologike epitheorese 58: 32-42. 
389. Rosengren, A., Smyth, A., Rangarajan, S., Ramasundarahettige, C., Bangdiwala, S.I., AlHabib, K.F., Avezum, A., Bengtsson Boström, K., Chifamba, J., Gulec, S., Gupta, R., Igumbor, E.U., Iqbal, R., Ismail, N., Joseph, P., Kaur, M., Khatib, R., Kruger, I.M., Lamelas, P., Lanas, F., Lear, S.A., Li, W., Wang, C., Quiang, D., Wang, Y., LopezJaramillo, P., Mohammadifard, N., Mohan, V., Mony, P.K., Poirier, P., Srilatha, S., Szuba, A., Teo, K., Wielgosz, A., Yeates, K.E., Yusoff, K., Yusuf, R., Yusufali, A.H., Attaei, M.W., McKee, M. and Yusuf, S. (2019). Socioeconomic status and risk of cardiovascular disease in 20 low-income, middle-income, and high-income countries: the Prospective Urban Rural Epidemiologic (PURE) study. The Lancet Global Health 7: e748-e760.

390. Zafar, N., Krishnasamy, S.S., Shah, J., Rai, S.N., Riggs, D.W., Bhatnagar, A. and O'Toole, T.E. (2018). Circulating angiogenic stem cells in type 2 diabetes are associated with glycemic control and endothelial dysfunction. PloS one 13: e0205851.

391. Brook, R.D., Bard, R.L., Kaplan, M.J., Yalavarthi, S., Morishita, M., Dvonch, J.T., Wang, L., Yang, H.-Y., Spino, C., Mukherjee, B., Oral, E.A., Sun, Q., Brook, J.R., Harkema, J. and Rajagopalan, S. (2013). The effect of acute exposure to coarse particulate matter air pollution in a rural location on circulating endothelial progenitor cells: results from a randomized controlled study. Inhalation toxicology 25: 587-592.

392. Lee, Y.O., Hebert, C.J., Nonnemaker, J.M. and Kim, A.E. (2014). Multiple tobacco product use among adults in the United States: cigarettes, cigars, electronic cigarettes, hookah, smokeless tobacco, and snus. Preventive medicine 62: 14-19.

393. Mirbolouk, M., Charkhchi, P., Kianoush, S., Uddin, S.M.I., Orimoloye, O.A., Jaber, R., Bhatnagar, A., Benjamin, E.J., Hall, M.E., DeFilippis, A.P., Maziak, W., Nasir, K. and Blaha, M.J. (2018). Prevalence and Distribution of E-Cigarette Use Among U.S. Adults: Behavioral Risk Factor Surveillance System, 2016. Annals of internal medicine 169: 429-438.

394. U.S. Department of Health and Human Services. (2016). Patterns of E-Cigarette Use Among U.S. Youth and Young Adults, in E-Cigarette Use Among Youth and Young Adults: A Report of the Surgeon General National Center for Chronic Disease Prevention and Health Promotion (US) Office on Smoking and Health, Atlanta, GA.

395. Keith, R.J., Fetterman, J.L., Orimoloye, O.A., Dardari, Z., Lorkiewicz, P.K., Hamburg, N.M., DeFilippis, A.P., Blaha, M.J. and Bhatnagar, A. (2019).

Characterization of Volatile Organic Compound Metabolites in Cigarette Smokers, Electronic Nicotine Device Users, Dual Users, and Nonusers of Tobacco. Nicotine \& Tobacco Research.

396. Smith, C.J. and Fischer, T.H. (2001). Particulate and vapor phase constituents of cigarette mainstream smoke and risk of myocardial infarction. Atherosclerosis 158: 257267.

397. Electronic cigarettes. 2018. Available from: www.cdc.gov2018. 
398. World Health Organization. (2017). WHO report on the global tobacco epidemic, 2017: monitoring tobacco use and prevention policies, World Health Organization, Geneva.

399. Hitchman, S., Craig, L., Driezen, P., Bishop, M., Fong, G.T. and Bhatti, L.I. (2012). Cardiovascular harms from tobacco use and secondhand smoke: Global gaps in awareness and implications for action, (Project, I., Organization, W.H. and Federation, W.H., eds), World Health Organization, Geneva, Switzerland.

400. Herrington, J.S. and Myers, C. (2015). Electronic cigarette solutions and resultant aerosol profiles. Journal of chromatography. A 1418: 192-199.

401. Benowitz, N.L. and Burbank, A.D. (2016). Cardiovascular toxicity of nicotine: Implications for electronic cigarette use. Trends in cardiovascular medicine 26: 515-523.

402. Strongin, R.M. (2019). E-Cigarette Chemistry and Analytical Detection. Annu Rev Anal Chem (Palo Alto Calif) 12: 23-39.

403. Garten, S. and Falkner, R.V. (2004). Role of mentholated cigarettes in increased nicotine dependence and greater risk of tobacco-attributable disease. Preventive medicine 38: 793-798.

404. Lin, A.-H., Liu, M.-H., Ko, H.-K., Perng, D.-W., Lee, T.-S. and Kou, Y.R. (2018). Menthol Cigarette Smoke Induces More Severe Lung Inflammation Than Nonmenthol Cigarette Smoke Does in Mice With Subchronic Exposure - Role of TRPM8. Frontiers in Physiology 9.

405. Ha, M.A., Smith, G.J., Cichocki, J.A., Fan, L., Liu, Y.-S., Caceres, A.I., Jordt, S.E. and Morris, J.B. (2015). Menthol Attenuates Respiratory Irritation and Elevates Blood Cotinine in Cigarette Smoke Exposed Mice. PloS one 10: e0117128.

406. Benowitz, N.L., Herrera, B. and Jacob, P., 3rd. (2004). Mentholated cigarette smoking inhibits nicotine metabolism. The Journal of pharmacology and experimental therapeutics 310: 1208-1215.

407. Leventhal, A.M., Miech, R., Barrington-Trimis, J., Johnston, L.D., O’Malley, P.M. and Patrick, M.E. (2019). Flavors of e-Cigarettes Used by Youths in the United States. JAMA : the journal of the American Medical Association 322: 2132-2134.

408. Wannamethee, S.G., Lowe, G.D.O., Shaper, A.G., Rumley, A., Lennon, L. and Whincup, P.H. (2005). Associations between cigarette smoking, pipe/cigar smoking, and smoking cessation, and haemostatic and inflammatory markers for cardiovascular disease. European heart journal 26: 1765-1773.

409. van Eeden, S.F. and Hogg, J.C. (2000). The response of human bone marrow to chronic cigarette smoking. European Respiratory Journal 15: 915. 
410. Venn, A. and Britton, J. (2007). Exposure to Secondhand Smoke and Biomarkers of Cardiovascular Disease Risk in Never-Smoking Adults. Circulation 115: 990-995.

411. Asgary, S., Naderi, G. and Ghannady, A. (2005). Effects of cigarette smoke, nicotine and cotinine on red blood cell hemolysis and their -SH capacity. Experimental and clinical cardiology 10: 116-119.

412. Sloan, A., Gona, P. and Johnson, A.D. (2015). Cardiovascular correlates of platelet count and volume in the Framingham Heart Study. Annals of epidemiology 25: 492-498.

413. Lakshmi, S.A., Lakshmanan, A., Kumar, P.G. and Saravanan, A. (2014). Effect of intensity of cigarette smoking on haematological and lipid parameters. Journal of clinical and diagnostic research : JCDR 8: BC11-BC13.

414. Khan, M.I., Bukhari, M.H., Akhtar, M.S. and Brar, S. (2014). Effect of smoking on red blood cells count, hemoglobin concentration and red cell indices. Pakistan Journal of Medical and Health Sciences 8: 361-364.

415. Layden, J.E., Ghinai, I., Pray, I., Kimball, A., Layer, M., Tenforde, M., Navon, L., Hoots, B., Salvatore, P.P., Elderbrook, M., Haupt, T., Kanne, J., Patel, M.T., SaathoffHuber, L., King, B.A., Schier, J.G., Mikosz, C.A. and Meiman, J. (2019). Pulmonary Illness Related to E-Cigarette Use in Illinois and Wisconsin - Preliminary Report. New Engl J Med.

416. Muthumalage, T., Prinz, M., Ansah, K.O., Gerloff, J., Sundar, I.K. and Rahman, I. (2018). Inflammatory and Oxidative Responses Induced by Exposure to Commonly Used e-Cigarette Flavoring Chemicals and Flavored e-Liquids without Nicotine. Frontiers in Physiology 8.

417. Regitz-Zagrosek, V., Oertelt-Prigione, S., Prescott, E., Franconi, F., Gerdts, E., Foryst-Ludwig, A., Maas, A.H.E.M., Kautzky-Willer, A., Knappe-Wegner, D., Kintscher, U., Ladwig, K.H., Schenck-Gustafsson, K. and Stangl, V. (2015). Gender in cardiovascular diseases: impact on clinical manifestations, management, and outcomes. European heart journal 37: 24-34.

418. Albrektsen, G., Heuch, I., Løchen, M.-L., Thelle, D.S., Wilsgaard, T., Njølstad, I. and Bønaa, K.H. (2016). Lifelong Gender Gap in Risk of Incident Myocardial Infarction: The Troms $\varnothing$ Study. JAMA Internal Medicine 176: 1673-1679.

419. Madsen, T.E., Howard, G., Kleindorfer, D.O., Furie, K.L., Oparil, S., Manson, J.E., Liu, S. and Howard, V.J. (2019). Sex Differences in Hypertension and Stroke Risk in the REGARDS Study: A Longitudinal Cohort Study. Hypertension 74: 749-755.

420. Chaudhary, R., Sukhi, A., Chaudhary, R., Jindal, M., Vyas, A., Rout, A., Bliden, K., Tantry, U. and Gurbel, P. (2019). Gender differences in thrombogenicity among patients with angina and non-obstructive coronary artery disease. Journal of thrombosis and thrombolysis 48: 373-381. 
421. Poorthuis, M.H., Algra, A.M., Algra, A., Kappelle, L.J. and Klijn, C.J. (2017). Female- and Male-Specific Risk Factors for Stroke: A Systematic Review and Metaanalysis. JAMA neurology 74: 75-81.

422. Spychala, M.S., Honarpisheh, P. and McCullough, L.D. (2017). Sex differences in neuroinflammation and neuroprotection in ischemic stroke. Journal of neuroscience research 95: 462-471.

423. Abdullah Shuaib, M., Defina Laura, F., Leonard, D., Barlow Carolyn, E., Radford Nina, B., Willis Benjamin, L., Rohatgi, A., McGuire Darren, K., de Lemos James, A., Grundy Scott, M., Berry Jarett, D. and Khera, A. (2018). Long-Term Association of Low-Density Lipoprotein Cholesterol With Cardiovascular Mortality in Individuals at Low 10-Year Risk of Atherosclerotic Cardiovascular Disease. Circulation 138: 23152325.

424. Peters, S.A.E., Singhateh, Y., Mackay, D., Huxley, R.R. and Woodward, M. (2016). Total cholesterol as a risk factor for coronary heart disease and stroke in women compared with men: A systematic review and meta-analysis. Atherosclerosis 248: 123131.

425. Allen, A.M., Oncken, C. and Hatsukami, D. (2014). Women and Smoking: The Effect of Gender on the Epidemiology, Health Effects, and Cessation of Smoking. Curr Addict Rep 1: 53-60.

426. Syamlal, G., Mazurek, J.M. and Dube, S.R. (2014). Gender differences in smoking among U.S. working adults. Am J Prev Med 47: 467-475.

427. Piñeiro, B., Correa, J.B., Simmons, V.N., Harrell, P.T., Menzie, N.S., Unrod, M., Meltzer, L.R. and Brandon, T.H. (2016). Gender differences in use and expectancies of ecigarettes: Online survey results. Addict Behav 52: 91-97.

428. Rodríguez-Bolaños, R., Arillo-Santillán, E., Barrientos-Gutiérrez, I., ZavalaArciniega, L., Ntansah, C.A. and Thrasher, J.F. (2019). Sex Differences in Becoming a Current Electronic Cigarette User, Current Smoker and Current Dual User of Both Products: A Longitudinal Study among Mexican Adolescents. International journal of environmental research and public health 17: 196.

429. Kong, G., Kuguru, K.E. and Krishnan-Sarin, S. (2017). Gender Differences in U.S. Adolescent E-Cigarette Use. Curr Addict Rep 4: 422-430.

430. Patel, J.D., Bach, P.B. and Kris, M.G. (2004). Lung Cancer in US Women: A Contemporary Epidemic. JAMA : the journal of the American Medical Association 291: 1763-1768.

431. Goniewicz, M.L., Smith, D.M., Edwards, K.C., Blount, B.C., Caldwell, K.L., Feng, J., Wang, L., Christensen, C., Ambrose, B., Borek, N., van Bemmel, D., Konkel, K., Erives, G., Stanton, C.A., Lambert, E., Kimmel, H.L., Hatsukami, D., Hecht, S.S., Niaura, R.S., Travers, M., Lawrence, C. and Hyland, A.J. (2018). Comparison of 
Nicotine and Toxicant Exposure in Users of Electronic Cigarettes and Combustible Cigarettes. JAMA Network Open 1: e185937-e185937.

432. Lindner, D., Smith, S., Leroy, C.M. and Tricker, A.R. (2011). Comparison of Exposure to Selected Cigarette Smoke Constituents in Adult Smokers and Nonsmokers in a European, Multicenter, Observational Study. Cancer Epidemiology Biomarkers \&amp; Prevention 20: 1524-1536.

433. Szumska, M., Damasiewicz-Bodzek, A. and Tyrpien-Golder, K. (2015). Environmental tobacco smoke--assessment of formaldehyde concentration in urine samples of exposed medicine students. Przeglad lekarski 72: 140-143.

434. Bagchi, M., Bagchi, D., Hassoun, E.A. and Stohs, S.J. (1998). Subchronic effects of smokeless tobacco extract (STE) on hepatic lipid peroxidation, DNA damage and excretion of urinary metabolites in rats. Toxicology 127: 29-38.

435. Khlystov, A. and Samburova, V. (2016). Flavoring Compounds Dominate Toxic Aldehyde Production during E-Cigarette Vaping. Environmental Science \& Technology 50: $13080-13085$.

436. Health, N.C.f.C.D.P.a.H.P.U.O.o.S.a. (2016). E-Cigarette Use Among Youth and Young Adults: A Report of the Surgeon General, (Prevention, C.f.D.C.a., ed), Atlanta, GA.

437. Vargová, M., Wagnerová, J., Lísková, A., Jakubovský, J., Gajdová, M., Stolcová, E., Kubová, J., Tulinská, J. and Stenclová, R. (1993). Subacute immunotoxicity study of formaldehyde in male rats. Drug and chemical toxicology 16: 255-275.

438. Yu, P.H. and Deng, Y.L. (1998). Endogenous formaldehyde as a potential factor of vulnerability of atherosclerosis: involvement of semicarbazide-sensitive amine oxidase-mediated methylamine turnover. Atherosclerosis 140: 357-363.

439. Li, S.-Y., Gomelsky, M., Duan, J., Zhang, Z., Gomelsky, L., Zhang, X., Epstein, P.N. and Ren, J. (2004). Overexpression of aldehyde dehydrogenase-2 (ALDH2) transgene prevents acetaldehyde-induced cell injury in human umbilical vein endothelial cells: role of ERK and p38 mitogen-activated protein kinase. The Journal of biological chemistry 279 : 11244-11252.

440. Sorg, B.A., Swindell, S. and Tschirgi, M.L. (2004). Repeated low level formaldehyde exposure produces enhanced fear conditioning to odor in male, but not female, rats. Brain Research 1008: 11-19.

441. Arthur, M.J., Lee, A. and Wright, R. (1984). Sex differences in the metabolism of ethanol and acetaldehyde in normal subjects. Clinical science (London, England : 1979) 67: 397-401.

442. Quintanilla, M.E., Tampier, L., Sapag, A., Gerdtzen, Z. and Israel, Y. (2007). Sex differences, alcohol dehydrogenase, acetaldehyde burst, and aversion to ethanol in the rat: 
a systems perspective. American Journal of Physiology-Endocrinology and Metabolism 293: E531-E537.

443. Fukunaga, T., Sillanaukee, P. and Eriksson, C.J. (1993). Occurrence of blood acetaldehyde in women during ethanol intoxication: preliminary findings. Alcoholism, clinical and experimental research 17: 1198-1200.

444. Chrostek, L., Jelski, W., Szmitkowski, M. and Puchalski, Z. (2003). Genderrelated differences in hepatic activity of alcohol dehydrogenase isoenzymes and aldehyde dehydrogenase in humans. J Clin Lab Anal 17: 93-96.

445. Maronpot, R.R., Miller, R.A., Clarke, W.J., Westerberg, R.B., Decker, J.R. and Moss, O.R. (1986). Toxicity of formaldehyde vapor in B6C3F1 mice exposed for 13 weeks. Toxicology 41: 253-266.

446. Til, H.P., Woutersen, R.A., Feron, V.J. and Clary, J.J. (1988). Evaluation of the oral toxicity of acetaldehyde and formaldehyde in a 4-week drinking-water study in rats. Food and Chemical Toxicology 26: 447-452.

447. Woutersen, R.A., Appelman, L.M., Wilmer, J.W., Falke, H.E. and Feron, V.J. (1987). Subchronic (13-week) inhalation toxicity study of formaldehyde in rats. Journal of applied toxicology : JAT 7: 43-49.

448. Til, H.P., Woutersen, R.A., Feron, V.J., Hollanders, V.H., Falke, H.E. and Clary, J.J. (1989). Two-year drinking-water study of formaldehyde in rats. Food and chemical toxicology : an international journal published for the British Industrial Biological Research Association 27: 77-87.

449. Rusch, G.M., Clary, J.J., Rinehart, W.E. and Bolte, H.F. (1983). A 26-week inhalation toxicity study with formaldehyde in the monkey, rat, and hamster. Toxicology and applied pharmacology 68: 329-343.

450. Sithu, S.D., Srivastava, S., Siddiqui, M.A., Vladykovskaya, E., Riggs, D.W., Conklin, D.J., Haberzettl, P., O'Toole, T.E., Bhatnagar, A. and D'Souza, S.E. (2010). Exposure to acrolein by inhalation causes platelet activation. Toxicology and applied pharmacology 248: 100-110.

451. Roemer, E., Schramke, H., Weiler, H., Buettner, A., Kausche, S., Weber, S., Berges, A., Stueber, M., Muench, M., Trelles-Sticken, E., Pype, J., Kohlgrueber, K., Voelkel, H. and Wittke, S. (2012). Mainstream Smoke Chemistry and in Vitro and In Vivo Toxicity of the Reference Cigarettes 3R4F and 2R4F. Beiträge zur Tabakforschung International/Contributions to Tobacco Research 25: 316-335.

452. Wang, L., Yang, Z., Xu, L., Pan, X., Liu, X., Zhao, J., Li, X., Zhu, M. and Xie, J. (2018). Acute exposure to crotonaldehyde induces dysfunction of immune system in male Wistar rats. The Journal of toxicological sciences 43: 33-44. 
453. Yang, B.C., Yang, Z.H., Pan, X.J., Liu, X.Y., Zhu, M.X. and Xie, J.P. (2013). Crotonaldehyde induces apoptosis and immunosuppression in alveolar macrophages. Toxicology in Vitro 27: 128-137.

454. Lambert, C., McCue, J., Portas, M., Ouyang, Y., Li, J., Rosano, T.G., Lazis, A. and Freed, B.M. (2005). Acrolein in cigarette smoke inhibits T-cell responses. The Journal of allergy and clinical immunology 116: 916-922.

455. Jeppesen, J., Hein, H.O., Suadicani, P. and Gyntelberg, F. (1998). Triglyceride concentration and ischemic heart disease: an eight-year follow-up in the Copenhagen Male Study. Circulation 97: 1029-1036.

456. Sarwar, N., Danesh, J., Eiriksdottir, G., Sigurdsson, G., Wareham, N., Bingham, S., Boekholdt, S.M., Khaw, K.T. and Gudnason, V. (2007). Triglycerides and the risk of coronary heart disease: 10,158 incident cases among 262,525 participants in 29 Western prospective studies. Circulation 115: 450-458.

457. Wang, L., Gill, R., Pedersen, T.L., Higgins, L.J., Newman, J.W. and Rutledge, J.C. (2009). Triglyceride-rich lipoprotein lipolysis releases neutral and oxidized FFAs that induce endothelial cell inflammation. J Lipid Res 50: 204-213.

458. Kajikawa, M., Maruhashi, T., Matsumoto, T., Iwamoto, Y., Iwamoto, A., Oda, N., Kishimoto, S., Matsui, S., Aibara, Y., Hidaka, T., Kihara, Y., Chayama, K., Goto, C., Noma, K., Nakashima, A., Tomiyama, H., Takase, B., Yamashina, A. and Higashi, Y. (2016). Relationship between serum triglyceride levels and endothelial function in a large community-based study. Atherosclerosis 249: 70-75.

459. Lucero, D., Lopez, G.I., Gorzalczany, S., Duarte, M., Gonzalez Ballerga, E., Sorda, J., Schreier, L. and Zago, V. (2016). Alterations in triglyceride rich lipoproteins are related to endothelial dysfunction in metabolic syndrome. Clinical biochemistry 49: 932-935.

460. Rapp, J.H., Lespine, A., Hamilton, R.L., Colyvas, N., Chaumeton, A.H., TweedieHardman, J., Kotite, L., Kunitake, S.T., Havel, R.J. and Kane, J.P. (1994). Triglyceriderich lipoproteins isolated by selected-affinity anti-apolipoprotein B immunosorption from human atherosclerotic plaque. Arteriosclerosis and thrombosis : a journal of vascular biology 14: 1767-1774.

461. Bessac, B.F., Sivula, M., von Hehn, C.A., Escalera, J., Cohn, L. and Jordt, S.E. (2008). TRPA1 is a major oxidant sensor in murine airway sensory neurons. The Journal of clinical investigation 118: 1899-1910.

462. Sawada, Y., Hosokawa, H., Matsumura, K. and Kobayashi, S. (2008). Activation of transient receptor potential ankyrin 1 by hydrogen peroxide. Eur J Neurosci 27: 11311142.

463. Obal, D., Dai, S., Keith, R., Dimova, N., Kingery, J., Zheng, Y.T., Zweier, J., Velayutham, M., Prabhu, S.D., Li, Q., Conklin, D., Yang, D., Bhatnagar, A., Bolli, R. 
and Rokosh, G. (2012). Cardiomyocyte-restricted overexpression of extracellular superoxide dismutase increases nitric oxide bioavailability and reduces infarct size after ischemia/reperfusion. Basic research in cardiology 107: 305.

464. Jun, S., Fattman, C.L., Kim, B.J., Jones, H. and Dory, L. (2011). Allele-specific effects of ecSOD on asbestos-induced fibroproliferative lung disease in mice. Free Radic Biol Med 50: 1288-1296.

465. Haberzettl, P., O'Toole, T.E., Bhatnagar, A. and Conklin, D.J. (2016). Exposure to Fine Particulate Air Pollution Causes Vascular Insulin Resistance by Inducing Pulmonary Oxidative Stress. Environ Health Perspect 124: 1830-1839.

466. Zhao, J. and Hopke, P.K. (2012). Concentration of Reactive Oxygen Species (ROS) in Mainstream and Sidestream Cigarette Smoke. Aerosol Science and Technology 46: 191-197.

467. Valavanidis, A., Vlachogianni, T. and Fiotakis, K. (2009). Tobacco smoke: involvement of reactive oxygen species and stable free radicals in mechanisms of oxidative damage, carcinogenesis and synergistic effects with other respirable particles. International journal of environmental research and public health 6: 445-462.

468. Huang, M.F., Lin, W.L. and Ma, Y.C. (2005). A study of reactive oxygen species in mainstream of cigarette. Indoor air 15: 135-140.

469. Miljevic, B., Fairfull-Smith, K.E., Bottle, S.E. and Ristovski, Z.D. (2010). The application of profluorescent nitroxides to detect reactive oxygen species derived from combustion-generated particulate matter: Cigarette smoke - A case study. Atmospheric Environment 44: 2224-2230.

470. $\quad$ van der Toorn, M., Rezayat, D., Kauffman, H.F., Bakker, S.J.L., Gans, R.O.B., Koëter, G.H., Choi, A.M.K., van Oosterhout, A.J.M. and Slebos, D.-J. (2009). Lipidsoluble components in cigarette smoke induce mitochondrial production of reactive oxygen species in lung epithelial cells. American Journal of Physiology-Lung Cellular and Molecular Physiology 297: L109-L114.

471. Morris, P.B., Ference, B.A., Jahangir, E., Feldman, D.N., Ryan, J.J., Bahrami, H., El-Chami, M.F., Bhakta, S., Winchester, D.E., Al-Mallah, M.H., Sanchez Shields, M., Deedwania, P., Mehta, L.S., Phan, B.A.P. and Benowitz, N.L. (2015). Cardiovascular Effects of Exposure to Cigarette Smoke and Electronic Cigarettes: Clinical Perspectives From the Prevention of Cardiovascular Disease Section Leadership Council and Early Career Councils of the American College of Cardiology. Journal of the American College of Cardiology 66: 1378-1391.

472. Kim, M., Han, C.-H. and Lee, M.-Y. (2014). NADPH oxidase and the cardiovascular toxicity associated with smoking. Toxicol Res 30: 149-157. 
473. De Rosa, S., Pacileo, M., Sasso, L., Di Palma, V., Maietta, P., Paglia, A., Brevetti, L., Cirillo, P. and Chiariello, M. (2008). Insights into pathophysiology of smoke-related cardiovascular disease. Monaldi Arch Chest Dis 70: 59-67.

474. Orosz, Z., Csiszar, A., Labinskyy, N., Smith, K., Kaminski, P.M., Ferdinandy, P., Wolin, M.S., Rivera, A. and Ungvari, Z. (2007). Cigarette smoke-induced proinflammatory alterations in the endothelial phenotype: role of $\mathrm{NAD}(\mathrm{P}) \mathrm{H}$ oxidase activation. American journal of physiology. Heart and circulatory physiology 292: H130H139.

475. Jung, O., Marklund, S.L., Geiger, H., Pedrazzini, T., Busse, R. and Brandes, R.P. (2003). Extracellular superoxide dismutase is a major determinant of nitric oxide bioavailability: in vivo and ex vivo evidence from ecSOD-deficient mice. Circ Res 93: 622-629.

476. Perveen, S., Patel, H., Arif, A., Younis, S., Codipilly, C.N. and Ahmed, M. (2012). Role of EC-SOD overexpression in preserving pulmonary angiogenesis inhibited by oxidative stress. PloS one 7: e51945-e51945.

477. Yao, H., Arunachalam, G., Hwang, J.W., Chung, S., Sundar, I.K., Kinnula, V.L., Crapo, J.D. and Rahman, I. (2010). Extracellular superoxide dismutase protects against pulmonary emphysema by attenuating oxidative fragmentation of ECM. Proceedings of the National Academy of Sciences of the United States of America 107: 15571-15576.

478. Foronjy, R.F., Mirochnitchenko, O., Propokenko, O., Lemaitre, V., Jia, Y., Inouye, M., Okada, Y. and D'Armiento, J.M. (2006). Superoxide Dismutase Expression Attenuates Cigarette Smoke- or Elastase-generated Emphysema in Mice. American journal of respiratory and critical care medicine 173: 623-631.

479. Wang, M., Zhang, Y., Xu, M., Zhang, H., Chen, Y., Chung, K.F., Adcock, I.M. and Li, F. (2019). Roles of TRPA1 and TRPV1 in cigarette smoke -induced airway epithelial cell injury model. Free radical biology \& medicine 134: 229-238.

480. Nassini, R., Pedretti, P., Moretto, N., Fusi, C., Carnini, C., Facchinetti, F., Viscomi, A.R., Pisano, A.R., Stokesberry, S., Brunmark, C., Svitacheva, N., McGarvey, L., Patacchini, R., Damholt, A.B., Geppetti, P. and Materazzi, S. (2012). Transient Receptor Potential Ankyrin 1 Channel Localized to Non-Neuronal Airway Cells Promotes Non-Neurogenic Inflammation. PloS one 7: e42454.

481. Lynch, J., Jin, L., Richarson, A., Jagatheesan, G., Lorkiewicz, P., Xie, Z., Malovichko, M., Bhatnagar, A., Srivastava, S. and Conklin, D.J. Vascular Effects of Inhaled Crotonaldehyde in Mice: Role of TRPA1. In preparation.

482. Jin, L., Jagatheesan, G., Lynch, J., Guo, L. and Conklin, D.J. (2020). Crotonaldehyde-induced vascular relaxation and toxicity: Role of endothelium and transient receptor potential ankyrin-1 (TRPA1). Toxicology and applied pharmacology: 115012. 
483. Beauchamp, R.O., Jr., Andjelkovich, D.A., Kligerman, A.D., Morgan, K.T. and Heck, H.D. (1985). A critical review of the literature on acrolein toxicity. Crit Rev Toxicol 14: 309-380.

484. Bolego, C., Poli, A. and Paoletti, R. (2002). Smoking and gender. Cardiovascular research 53: 568-576.

485. Patil, M.J., Green, D.P., Henry, M.A. and Akopian, A.N. (2013). Sex-dependent roles of prolactin and prolactin receptor in postoperative pain and hyperalgesia in mice. Neuroscience 253: 132-141.

486. Patil, M.J., Ruparel, S.B., Henry, M.A. and Akopian, A.N. (2013). Prolactin regulates TRPV1, TRPA1, and TRPM8 in sensory neurons in a sex-dependent manner: Contribution of prolactin receptor to inflammatory pain. Am J Physiol Endocrinol Metab 305: E1154-E1164.

487. Ferrari, R., Ceconi, C., Curello, S., Ghielmi, S. and Albertini, A. (1989). Superoxide dismutase: Possible therapeutic use in cardiovascular disease. Pharmacological research 21: 57-65.

488. Schiffrin, E.L. (2008). Oxidative Stress, Nitric Oxide Synthase, and Superoxide Dismutase. Hypertension 51: 31-32.

489. Alarie, Y. (1973). Sensory irritation of the upper airways by airborne chemicals. Toxicology and applied pharmacology 24: 279-297.

490. McNamara, C.R., Mandel-Brehm, J., Bautista, D.M., Siemens, J., Deranian, K.L., Zhao, M., Hayward, N.J., Chong, J.A., Julius, D., Moran, M.M. and Fanger, C.M. (2007). TRPA1 mediates formalin-induced pain. Proceedings of the National Academy of Sciences of the United States of America 104: 13525-13530.

491. Bang, S., Kim, K.Y., Yoo, S., Kim, Y.G. and Hwang, S.W. (2007). Transient receptor potential A1 mediates acetaldehyde-evoked pain sensation. Eur J Neurosci 26: 2516-2523.

492. Jin, L., Lorkiewicz, P., Malovichko, M.V., Bhatnagar, A., Srivastava, S. and Conklin, D.J. (2019). Acetaldehyde Induces an Endothelium-Dependent Relaxation of Superior Mesenteric Artery: Potential Role in Postprandial Hyperemia. Frontiers in physiology 10: 1315-1315.

493. Nonaka-Sarukawa, M., Yamamoto, K., Aoki, H., Nishimura, Y., Tomizawa, H., Ichida, M., Eizawa, T., Muroi, K., Ikeda, U. and Shimada, K. (2007). Circulating endothelial progenitor cells in congestive heart failure. International journal of cardiology 119: 344-348.

494. Werner, N., Kosiol, S., Schiegl, T., Ahlers, P., Walenta, K., Link, A., Böhm, M. and Nickenig, G. (2005). Circulating Endothelial Progenitor Cells and Cardiovascular Outcomes. New Engl J Med 353: 999-1007. 
495. Xiao, Q., Kiechl, S., Patel, S., Oberhollenzer, F., Weger, S., Mayr, A., Metzler, B., Reindl, M., Hu, Y., Willeit, J. and Xu, Q. (2007). Endothelial progenitor cells, cardiovascular risk factors, cytokine levels and atherosclerosis--results from a large population-based study. PloS one 2: e975-e975.

496. Jaber, R.M., Mirbolouk, M., DeFilippis, A.P., Maziak, W., Keith, R., Payne, T., Stokes, A., Benjamin, E., Bhatnagar, A., Blankstein, R., Saxena, A., Blaha, M.J. and Nasir, K. (2018). Electronic Cigarette Use Prevalence, Associated Factors, and Pattern by Cigarette Smoking Status in the United States From NHANES (National Health and Nutrition Examination Survey) 2013\&\#x2013;2014. Journal of the American Heart Association 7: e008178.

497. Coleman, B.N., Rostron, B., Johnson, S.E., Ambrose, B.K., Pearson, J., Stanton, C.A., Wang, B., Delnevo, C., Bansal-Travers, M., Kimmel, H.L., Goniewicz, M.L., Niaura, R., Abrams, D., Conway, K.P., Borek, N., Compton, W.M. and Hyland, A. (2017). Electronic cigarette use among US adults in the Population Assessment of Tobacco and Health (PATH) Study, 2013-2014. Tobacco control 26: e117-e126.

498. Adab, P., Pallan, M. and Whincup, P.H. (2018). Is BMI the best measure of obesity? BMJ (Clinical research ed.) 360: k1274.

499. Yusuf, S., Hawken, S., Ounpuu, S., Bautista, L., Franzosi, M.G., Commerford, P., Lang, C.C., Rumboldt, Z., Onen, C.L., Lisheng, L., Tanomsup, S., Wangai, P., Jr., Razak, F., Sharma, A.M. and Anand, S.S. (2005). Obesity and the risk of myocardial infarction in 27,000 participants from 52 countries: a case-control study. Lancet 366: 1640-1649.

500. van Dis, I., Kromhout, D., Geleijnse, J.M., Boer, J.M. and Verschuren, W.M. (2009). Body mass index and waist circumference predict both 10-year nonfatal and fatal cardiovascular disease risk: study conducted in 20,000 Dutch men and women aged 2065 years. European journal of cardiovascular prevention and rehabilitation : official journal of the European Society of Cardiology, Working Groups on Epidemiology \& Prevention and Cardiac Rehabilitation and Exercise Physiology 16: 729-734.

501. de Koning, L., Merchant, A.T., Pogue, J. and Anand, S.S. (2007). Waist circumference and waist-to-hip ratio as predictors of cardiovascular events: metaregression analysis of prospective studies. European heart journal 28: 850-856.

502. Poirier, P., Giles Thomas, D., Bray George, A., Hong, Y., Stern Judith, S., PiSunyer, F.X. and Eckel Robert, H. (2006). Obesity and Cardiovascular Disease: Pathophysiology, Evaluation, and Effect of Weight Loss. Circulation 113: 898-918.

503. Cercato, C. and Fonseca, F.A. (2019). Cardiovascular risk and obesity. Diabetology \& Metabolic Syndrome 11: 74.

504. Moghe, A., Ghare, S., Lamoreau, B., Mohammad, M., Barve, S., McClain, C. and Joshi-Barve, S. (2015). Molecular Mechanisms of Acrolein Toxicity: Relevance to Human Disease. Toxicological Sciences 143: 242-255. 


\section{ABBREVIATIONS}

\begin{tabular}{ll} 
AA & Acetaldehyde \\
AAMA & N-acetyl-S-(2-carbamoylethyl)-L-cysteine \\
ACE & Angiotensin-converting enzyme \\
ALDH2 & Aldehyde dehydrogenase \\
ALT & Alanine aminotransferase \\
AMCC & N-acetyl-S-(N-methylcarbamoyl)-L-cysteine \\
ANB & Anabasine \\
ANOVA & Analysis of variance \\
ANTB & Anatabine \\
AST & Aspartate aminotransferase \\
BMA & N-acetyl-S-(benzyl)-L-cysteine \\
BSA & Bovine serum albumin \\
BWT & Body weight \\
CABG & Coronary artery bypass grafting \\
CAC & Circulating angiogenic cell \\
CBC & Complete blood cell count \\
CDC & Centers for Disease Control and Prevention \\
CEMA & N-acetyl-S-(2-carboxyethyl)-L-cysteine \\
CIG & Cigarette \\
\hline
\end{tabular}




$\begin{array}{ll}\text { CIMT } & \text { Carotid intima-media thickness } \\ \text { CK } & \text { Creatinine kinase } \\ \text { COT } & \text { Cotinine } \\ \text { CR } & \text { Crotonaldehyde } \\ \text { CVD } & \text { Cardiovascular disease } \\ \text { DHBMA } & \text { N-acetyl-S-(3,4-dihydroxybutyl)-L-cysteine } \\ \text { e-cig } & \text { Electronic cigarette } \\ \text { ecSOD } & \text { Extracellular superoxide dismutase } \\ \text { ecSOD-Tg } & \text { Extracellular superoxide dismutase transgenic } \\ \text { ENDs } & \text { Electronic nicotinic delivery system } \\ \text { eNOS } & \text { Endothelial nitric oxide synthase } \\ \text { EPC } & \text { Endothelial progenitor cell } \\ \text { EVALI } & \text { E-cigarette, or vaping, product use associated lung injury } \\ \text { FACS } & \text { Florescence-activated cell sorting } \\ \text { FR } & \text { Formaldehyde } \\ \text { GC-MS } & \text { Gas chromatography/mass spectrometry } \\ \text { 3HC } & \text { Trans-3'-hydroxycotinine } \\ \text { HDL } & \text { High-density lipoprotein } \\ \text { HEPA } & \text { High-efficiency particulate air } \\ \text { 2HPMA } & \text { N-acetyl-S-(2-hydroxypropyl)-L-cysteine } \\ \text { HPMMA } & \text { 3-hydroxypropylmercapturic acid } \\ \text { H-hydroxy-1-methylpropylmercapturic acid } \\ \text { Highool }\end{array}$




\begin{tabular}{ll} 
KY & Kentucky \\
LDH & Lactate dehydrogenase \\
LDL & Low-density lipoprotein \\
LHHS & Louisville Healthy Heart Study \\
MA & Mandelic acid \\
MCS & Mainstream cigarette smoke \\
2MHA & 2-methylhippuric acid \\
3MHA & 3-methylhippuric acid \\
4MHA & 4-methylhippuric acid \\
3MHBMA & N-acetyl-S-(4-hydroxy-2-buten-1-yl)-L-cysteine \\
NHANES & National Health and Nutrition Examination Survey \\
NIC & Nicotine \\
NO & Nitric oxide \\
O/N & Overnight \\
PBS & Phosphate-buffered saline \\
PCI & Percutaneous coronary intervention \\
PG & Propylene glycol \\
PGA & Phenylglyoxylic acid \\
PM & Particulate matter \\
PMA & Platelet mononuclear cell aggregate \\
RDW & Red blood cell distribution width \\
REDCap & Research Electronic Data Capture \\
RHI & Reactive hyperemia index \\
\hline
\end{tabular}




$\begin{array}{ll}\text { ROS } & \text { Reactive oxygen species } \\ \text { SHS } & \text { Socioeconomic status } \\ \text { SOD } & \text { Secondhand smoke } \\ \text { TG } & \text { Superoxide dismutase } \\ \text { THC } & \text { Transgenic } \\ \text { Total NIC } & \text { Totrahydrocannabinol nicotine } \\ \text { TRPA1 } & \text { Transient receptor potential ankyrin 1 } \\ \text { TSP } & \text { Total suspended particulate } \\ \text { UPLC-MS/MS } & \text { Ultra-performance liquid chromatography tandem mass spectrometry } \\ \text { VA } & \text { Virginia } \\ \text { VG } & \text { Vegetable glycerin } \\ \text { VOCs } & \text { Volatile organic compounds } \\ \text { WT } & \text { Wild type }\end{array}$




\title{
CURRICULUM VITAE
}

\author{
Jordan B. Lynch \\ University of Louisville School of Medicine \\ Department of Pharmacology and Toxicology \\ Louisville, KY 40202 \\ jordan.finch@louisville.edu \\ 636-232-6181
}

\section{EDUCATION}

2013-2016 M.S. in Pharmacology and Toxicology; University of Louisville, Louisville, KY

2011-2013 M.S. in Biological Sciences; Southern Illinois University Edwardsville, Edwardsville, IL

2007-2011 B.S. in Biology, Minors in Chemistry and Biochemistry; McKendree University, Lebanon, IL

\section{PROFESSIONAL EXPERIENCE}

2013-Present Graduate Research Assistant, University of Louisville, Department of Pharmacology and Toxicology

2011-2013 Forensic Toxicology Laboratory Staff, Saint Louis University

$2010 \quad$ Forensic Toxicology Laboratory Staff, Saint Louis University

\section{PROFESSIONAL MEMBERSHIPS AND ACTIVITIES}

2016-Present Society of Toxicology, Student Member

2016-Present Ohio Valley Society of Toxicology

2013 Phi Kappa Phi Honor Society

2012-2013 Illinois State Academy of Science 
2008-2011 Sigma Zeta National Science and Mathematics Honor Society

\section{HONORS AND AWARDS}

2017

2017

2017

2016-2018

2013

2012

2011

2011

2011

2009-2011

2009-2011

2009-2011

2007-2011

2007-2011

2007-2008

SERVICE

2019-Present Sojourn Church East SojournKids Volunteer

2017-2019 Sojourn Church East Student Ministry Leader

\section{LEADERSHIP}

2010-2011 McKendree University Dance Team Captain
Ohio Valley Society of Toxicology Ph.D. Poster Presentation Award

University of Louisville School of Interdisciplinary and Graduate Studies Travel Award

Society of Toxicology Graduate Student Travel Support Award

NIEHS T32 Training Program in Environmental Health Sciences Pre-

Doctoral Training Grant

Illinois State Academy of Science Environmental Health Science Poster

Presentation Award

Southern Illinois University Edwardsville Research Grant for Graduate Students

McKendree University Honors Program Graduate

McKendree University Magna Cum laude

McKendree University Biology Department Top Student Award

McKendree University Dean's List

McKendree University Carrie Field Smith and Emma Lee Foree Walkup

Smith Memorial Scholarship

McKendree University Edwin P. Baker Scholarship

McKendree University Founder's Scholarship

McKendree University Dance Team Scholarship

McKendree University President's List 
2009-2011 Sigma Zeta National Science and Mathematics Honor Society Beta Chapter Vice President

\section{ABSTRACT AND PRESENTATIONS}

\section{Oral}

\section{Sigma Zeta National Science and Mathematics Honors Society National Convention}

- What to Do with a Dead Body: A Forensic Evaluation of Skeletal Remains. San Antonio, TX, 2011

Poster

\section{Superfund Research Program Annual Meeting}

- Jordan Finch\#, Stacey Konkle\#, Aruni Bhatnagar, Katlyn McGraw, Haley Metcalf, Jamar Wheeler, Lauren Heberle. What Do We Know? A Critical Review of Site Condition and Public Health Risk Assessments in EPA Five-Year Reviews of the Lee's Lane Superfund Site in Louisville, KY. Seattle, WA, 2019. \#, co-first authors.

\section{Society of Toxicology Annual Meeting}

- Jordan Finch and Daniel J. Conklin. Effects of Tobacco Product-Derived Unsaturated Aldehydes on Circulating Angiogenic Cells. Baltimore, MD, 2019.

- Jordan Finch, Andre Richardson, Lexiao Jin, Daniel J. Conklin. Effects of Tobacco Product-Derived Aldehydes on Circulating Angiogenic Cells-Implications for Endothelium Dysfunction and Repair. San Antonio, TX, 2018.

- Jordan Finch, Gary Hoyle, Connie Schlueter, Ian McKinley, Aruni Bhatnagar, Daniel J. Conklin. Cardiopulmonary Effects of Acute Exposure to Electronic Cigarettes and Formaldehyde in Mice. Baltimore, MD, 2017.

\section{Ohio Valley Society of Toxicology Regional Chapter Annual Meeting}

- Jordan Finch, Andre Richardson, Pawel Lorkiewicz, Zhengzhi Xie, Daniel J. Conklin. Effect of Tobacco Product-Derived Saturated and Unsaturated Aldehydes on Circulating Angiogenic Cells. Procter \& Gamble Mason Business Center, Mason, OH, 2019. 
- Jordan Finch and Daniel J. Conklin. Effects of Tobacco Product-Derived Unsaturated Aldehydes on Circulating Angiogenic Cells. University of Louisville, Louisville, KY, 2018.

- Jordan Finch, Andre Richardson, Lexiao Jin, Daniel J. Conklin. Effects of Tobacco Product-Derived Aldehydes on Circulating Angiogenic Cells-Implications for Endothelium Dysfunction and Repair. Purdue University, West Lafayette, IN, 2017.

- Jordan Finch, Gary Hoyle, Connie Schlueter, Ian McKinley, Aruni Bhatnagar, Daniel J. Conklin. Cardiopulmonary Effects of Acute Exposure to Electronic Cigarettes and Formaldehyde in Mice. Eli Lilly Corporate Center, Indianapolis, IN, 2016.

\section{University of Louisville Research!Louisville}

- Jordan Finch, Andre Richardson, Pawel Lorkiewicz, Zhengzhi Xie, Daniel J. Conklin. Effect of Tobacco Product-Derived Saturated and Unsaturated Aldehydes on Circulating Angiogenic Cells. Louisville, KY, 2019.

- Jordan Finch, Andre Richardson, Lexiao Jin, Daniel J. Conklin. Role of Crotonaldehyde in the Cardiovascular Toxicity of Mainstream Cigarette Smoke. Louisville, KY, 2018.

- Jordan Finch, Andre Richardson, Daniel J. Conklin. Role of Tobacco Smoke-Derived Aldehydes on Circulating Angiogenic Cells-Implications for Endothelium Repair. Louisville, KY, 2017

- Jordan Finch, Gary Hoyle, Connie Schlueter, Aruni Bhatnagar, Daniel J. Conklin. Electronic Cigarette Exposure and Pulmonary Function in Mice: Role of Formaldehyde. Louisville, KY, 2016.

- Jordan Finch, Petra Haberzettl, Aruni Bhatnagar, Daniel J. Conklin. Air Pollution, Pulmonary Oxidative Stress, and the Endothelin System in the Development of Cardiovascular Disease. Louisville, KY, 2015.

- Jordan Finch, Timothy E. O'Toole, James McCracken, Natasha DeJarnett, Aruni Bhatnagar, C. Arden Pope III, Daniel J. Conklin. Air Pollution and Biomarkers of Cardiovascular Injury -- Endothelin-1 and Endothelial Progenitor Cells in Humans. Louisville, KY, 2014. 


\section{University of Louisville Cardiovascular Research Symposium}

- Jordan Finch and Daniel J. Conklin. Effects of Tobacco Product-Derived Unsaturated Aldehydes on Circulating Angiogenic Cells. Louisville, KY, 2019.

\section{Illinois State Academy of Science Annual Meeting}

- Jordan Finch, Kurt Schulz, Richard Brugam, Zhi-Qing Lin, Luci Kohn. A Study in Lead: An Analysis of Human Lead Exposure in the Old Lead Belt of Southeastern Missouri. Jacksonville, IL, 2013.

\section{Publications}

- Lynch, J.\#, Jin, L.\#, Richardson, A., Jagatheesan, G., Lorkiewicz, P., Xie, Z., Malovichko, M., Bhatnagar, A., Srivastava, S., \& Conklin, D.J. Vascular Effects of Inhaled Crotonaldehyde in Mice: Role of TRPA1. In revision. \#, co-first authors.

- Jin, L., Jagatheesan, G., Lynch, J., Guo, L., \& Conklin, D.J. (2020). Crotonaldehydeinduced Vascular Relaxation and Toxicity: Role of Endothelium and Transient Receptor Potential Ankyrin 1 (TRPA1). Toxicology and Applied Pharmacology. In press.

- Finch, J., Riggs, D.W., O’Toole, T., Pope, C.A., 3rd, Bhatnagar, A., \& Conklin, D.J. (2019). Acute exposure to air pollution is associated with novel changes in blood levels of endothelin-1 and circulating angiogenic cells in young, healthy adults. AIMS Environmental Science, 6(4): 265-276.

- Yeager, R., Riggs, D.W., DeJarnett, N., Tollerud, D.J., Wilson, J., Conklin, D.J., O’Toole, T., McCracken, J., Lorkiewicz, P., Xie, Z., Zafar, N., Krishnasamy, S., Srivastava, S., Finch, J., Keith, R., DeFilippis, A., Rai, S., Liu, G., \& Bhatnagar, A. (2018). Association Between Residential Greenness and Cardiovascular Disease Risk. Journal of the American Heart Association. 7(24): e009117.

- Finch, J. \& Conklin, D. (2016). Air Pollution-Induced Vascular Dysfunction: Potential Role of Endothelin-1 (ET-1) System. Cardiovascular Toxicology, 16(3): 260-275.

\section{Scientific Writing}

- Lynch, J. \& Conklin, D. (2020). Aldehydes \& You: What You Should Know About Aldehydes and Cardiovascular Disease. Sustain Magazine, 42. 
- Lynch, J., Konkle, S., McGraw, K., Wheeler, J., Metcalf, H., \& Heberle, L. (2020). What is the Status of the Lee's Lane Superfund Site? Sustain Magazine, 42.

\section{Abstracts}

\section{American Heart Association Scientific Sessions}

- Conklin, D.J., Jin, L., Whitehouse, K., Holbrook, D., Finch, J., Lorkiewicz, P., Srivastava, S., Bhatnagar, A. (2019). Cardiorespiratory Responses to JUUL and Tobacco Cigarettes in Mice: Role of Acrolein.

\section{American Public Health Association Annual Meeting}

• DeJarnett, N., Yeager, R., Conklin, D.J., O’Toole, T., McCracken, J., Abplanalp, W., Riggs, D., Hamzeh, I., Wagner, S., Chugh, A., DeFilippis, A., Ciszewski, T., Wyatt, B., Becher, C., Hidgon, D., Finch, J., Ismail, I., Tollerud, D.J., Rai, S., Shah, J., Zafar, N., Krishnasamy, S., Prabhu, S., Bhatnagar, A. (2015). Air Pollution and Tobacco Smoke Differentially Depress Circulating Angiogenic Cells in Humans. 